\title{
DEMAND-SIDE LOAD MANAGEMENT USING SINGLE-PHASE RESIDENTIAL STATIC VAR COMPENSATORS
}

by

Muhammad Kamran Latif

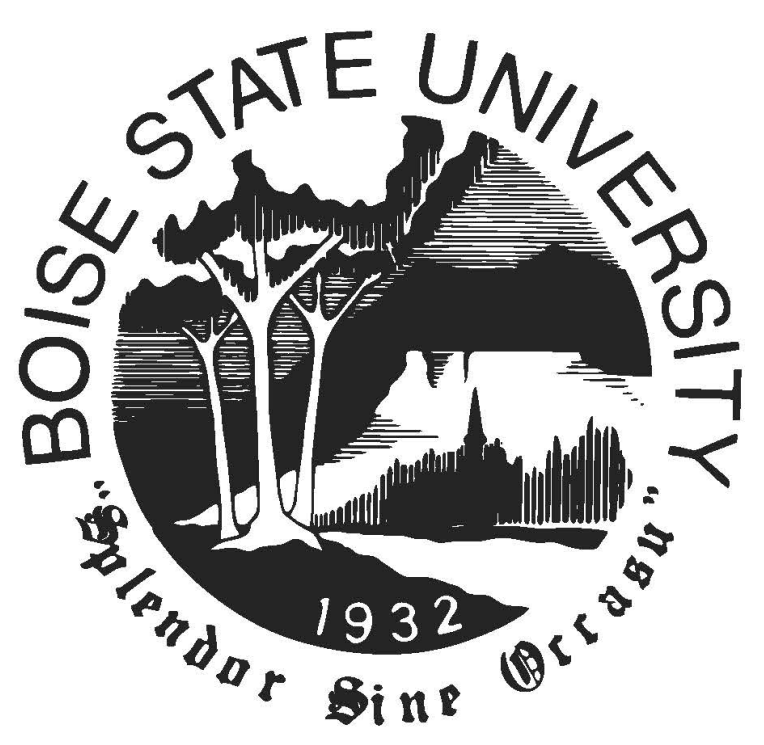

A dissertation

submitted in partial fulfillment

of the requirements for the degree of

Doctor of Philosophy in Electrical and Computer Engineering

Boise State University

December 2019 
(C) 2019

Muhammad Kamran Latif

ALL RIGHTS RESERVED 
BOISE STATE UNIVERSITY GRADUATE COLLEGE

\title{
DEFENSE COMMITTEE AND FINAL READING APPROVALS
}

\author{
of the Dissertation submitted by
}

Muhammad Kamran Latif

Dissertation Title: Demand-Side Load Management Using Single-Phase Residential Static VAR Compensators

Date of Oral Examination: 6th December 2019

The following individuals read and discussed the dissertation submitted by student Muhammad Kamran Latif, and they evaluated the presentation and response to questions during the final oral examination. They found that the student passed the final oral examination.

Said Ahmed-Zaid, Ph.D. Chair, Supervisory Committee

Nader Rafla, Ph.D. Co-Chair, Supervisory Committee

Thad Welch, Ph.D. Member, Supervisory Committee

John Stubban, Ph.D. Member, Supervisory Committee

Milorad Papic, Ph.D. $\quad$ External Examiner

The final reading approval of the Dissertation was granted by Said Ahmed-Zaid, Ph.D., Chair of the Supervisory Committee. The dissertation was approved by the Graduate College. 


\section{ACKNOWLEDGMENTS}

I sincerely thank my advisor, Dr. Said Ahmed-Zaid, for his supervision, guidance, and advice during the entire course of this research work. Dr. Ahmed-Zaid's unflinching encouragement and endless support inspired me during my stay at Boise State University (BSU). I would also like to thank my committee members Drs. Nader Rafla, Thad Welch and John Stubban for their guidance, constructive critique and advice during the project work.

Furthermore, I would like to acknowledge the Department of Electrical and Computer Engineering (ECE) at BSU for allowing me to use various departmental facilities that helped in carrying out the research efficiently as well as for giving me the opportunity to teach and instruct the Microprocessor class and lab during various semesters.

Additionally, I would also like to thank Avista Utilities for their support during this research. Especially, I would like to acknowledge Randy Gnaedinger and Reuben Arts at Avista Utilities for their support of this research project.

I am grateful to all the members of the research lab, especially, Danyal Mohammadi and Andrés Valdepeña for their continuous support and fruitful discussions. Moreover, I would like to extend my gratitude to Luka Daoud and Ziyang Liang with whom I have collaborated in several research projects.

Finally, I am extremely thankful to my family for their boundless love and unwavering support during my the Ph.D. journey. This work is dedicated to all of you. 


\begin{abstract}
Distribution systems are going through a structural transformation from being radiallyoperated simple systems to becoming more complex networks to operate in the presence of distributed energy resources (DERs) with significant levels of penetration. It is predicted that the share of electricity generation from DERs will keep increasing as the world is moving away from power generation involving carbon-emission and towards cleaner energy sources such as solar, wind, and biofuels. However, the variable behavior of renewable resources presents challenges to the already existing distribution systems. One such problem is when the distribution feeder experiences variable power supply due to the unpredictable behavior of renewable resources. Therefore, it becomes difficult to maintain end-of-line (EOL) voltages within an acceptable range of the ANSI C84.1 Standard.

Moreover, electric utility companies consider Conservation by Voltage Reduction (CVR) as a potential solution for managing peak power demand in distribution feeders. Conservation by Voltage Reduction is the implementation of a distribution voltage strategy whereby all distribution voltages are lowered to the minimum allowed by the equipment manufacturer. This strategy is rooted in the fact that many loads consume less power when they are fed with a voltage lower than nominal. Therefore, by implementing CVR, utility companies can potentially reduce the peak power demand and can delay the up-gradation of the distribution feeder assets. To maximize the benefits from CVR, the whole distribution feeder must participate in regulating power to lower the demand during hours of demand. Hence, there is a need for a local solution that can regulate residential voltage levels from
\end{abstract}


the first customer on the distribution feeder until the EOL of the distribution network. Such a solution will not only provide flexibility to electric utilities for better control over residential voltages but it can also maximize the benefits from CVR.

This dissertation presents the concept of a closed-loop Residential Static VAR Compensator (RSVC) that will allow electric utility companies to locally regulate the voltage across the distribution feeder. The proposed RSVC is a novel smart-grid device that can regulate a residential load voltage with a fixed capacitor in shunt with a reactor controlled by two bi-directional switches. The two switches are turned on and off in a complementary manner using a pulse-width modulation (PWM) technique that allows the reactor to function as a continuously-variable inductor. The proposed RSVC has several advantages compared to a conventional thyristor-based Static VAR Compensator (SVC), such as a quasi-sinusoidal inductor current, sub-cycle reactive power controllability, lower footprint for reactive components, and its realization as a single-phase device. The closed-loop RSVC contains two regulation control loops: the primary control loop regulates the customer load voltage to any desired reference voltage within ANSI C84.1 (120 V nominal $\pm 5 \%)$ and a secondary loop adjusting the reference voltage to track the point of minimum power consumption by the loads. This approach to CVR has the merit of adapting to the nature of the customer load, which may or may not decrease its energy consumption under a reduced voltage. This local approach to voltage regulation and CVR is a radical departure from current CVR strategies that have been in existence for over 30 years but have not been widely adopted by electric utilities due to high costs and technical challenges. 


\section{TABLE OF CONTENTS}

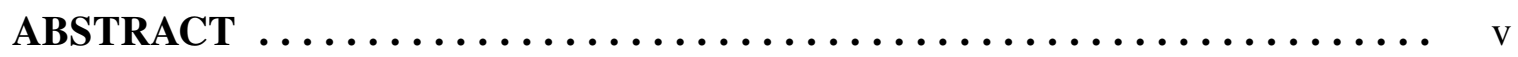

LIST OF TABLES $\ldots \ldots \ldots \ldots \ldots \ldots \ldots \ldots \ldots \ldots \ldots \ldots \ldots \ldots \ldots \ldots \ldots$ xiii

LIST OF FIGURES $\ldots \ldots \ldots \ldots \ldots \ldots \ldots \ldots \ldots \ldots \ldots \ldots \ldots \ldots \ldots \ldots \ldots \ldots$ xiv

LIST OF ABBREVIATIONS $\ldots \ldots \ldots \ldots \ldots \ldots \ldots \ldots \ldots \ldots \ldots \ldots$ xxii

1 Introduction $\ldots \ldots \ldots \ldots \ldots \ldots \ldots \ldots \ldots \ldots \ldots \ldots \ldots \ldots \ldots \ldots \ldots, 1$

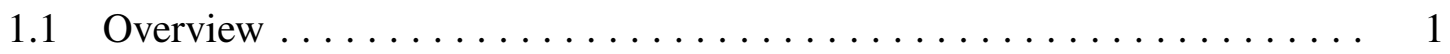

1.2 Research Motivation ........................... 8

1.2.1 Conservation by Voltage Reduction $(\mathrm{CVR}) \ldots \ldots \ldots \ldots \ldots \ldots$

1.2.2 Traditional Methods for CVR Implementation $\ldots \ldots \ldots \ldots \ldots \quad 12$

1.2.3 Minimum Power Point Tracker (MinPPT) / Optimal Operational

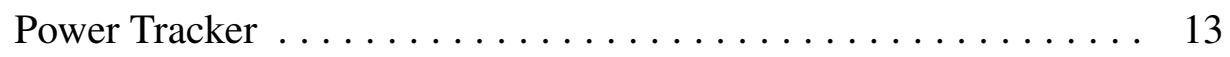

1.3 Proposed Solution - Residential Static VAR Compensator (RSVC) . . . . . 15

1.4 Organization of the Dissertation $\ldots \ldots \ldots \ldots \ldots \ldots \ldots \ldots \ldots \ldots \ldots \ldots$

2 Operating Principles of Residential Static VAR Compensators .......... 19

2.1 Fundamental Principles of AC Power Flow . . . . . . . . . . . . 19

2.2 Effect of Shunt Reactive Power Compensation ................. 21

2.3 Reactive Power Compensation Techniques ................... 25

2.4 Static VAR Compensators $(\mathrm{SVCs}) \ldots \ldots \ldots \ldots \ldots \ldots \ldots \ldots \ldots \ldots \ldots \ldots \ldots$ 
2.4.1 Different Configurations of SVCs . . . . . . . . . . . . . . . . 29

2.5 SVC Characteristics . . . . . . . . . . . . . . . . . . . . . 29

2.5.1 SVC Characteristics in a Power System . . . . . . . . . . . . 32

2.6 Thyristor-controlled reactor $(\mathrm{TCR}) \ldots \ldots \ldots \ldots \ldots \ldots \ldots \ldots \ldots \ldots$

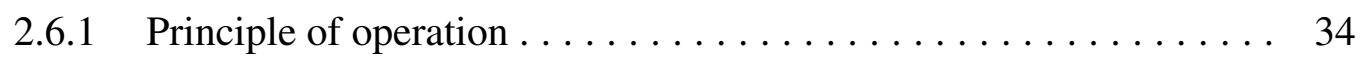

2.6.2 Harmonic Analysis for TCR Current $\ldots \ldots \ldots \ldots \ldots$

2.6.3 Three-Phase Thyristor-Controlled Reactor . . . . . . . . . . . . . . 40

2.7 Thyristor-Switched Reactor $(\mathrm{TSR}) \ldots \ldots \ldots \ldots \ldots \ldots \ldots \ldots$

2.8 Thyristor-Switched Capacitor $(\mathrm{TSC}) \ldots \ldots \ldots \ldots \ldots \ldots \ldots \ldots \ldots$

2.8.1 Principle of Operation $\ldots \ldots \ldots \ldots \ldots \ldots \ldots \ldots \ldots$

2.8.2 Dynamic Response with Multiple TSCs . . . . . . . . . . . . . 44

2.9 An Efficient Switched-Reactor-Based Static VAR Compensator . . . . . . . 47

2.9.1 PWM-Based Switched Reactor . . . . . . . . . . . . . . . . . . 47

2.9.2 Switching the Current Harmonic Distortion for the PWM-Based

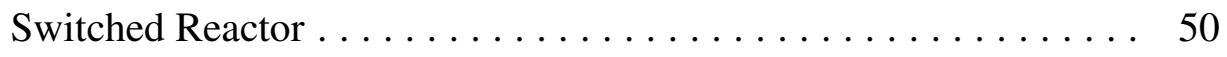

2.9.3 Input Current Harmonic Distortion for PWM-Based Switched Re-

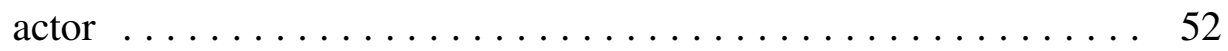

2.9.4 The Reactive Power Capability of a PWM-Based Switched Reactor with a Fixed Capacitor $\ldots \ldots \ldots \ldots \ldots \ldots \ldots$

2.9.5 Design Example...................... 54

\section{Bi-Directional Solid State Switches in Residential Static Var Compensators}

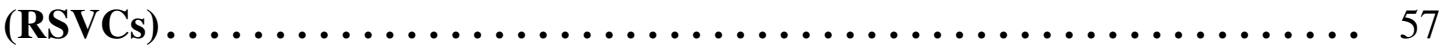

3.1 Bi-Directional Switches Topologies $\ldots \ldots \ldots \ldots \ldots \ldots \ldots \ldots \ldots$

3.2 Current Commutation Problem Between Bi-directional Switches . . . . . . 61 
3.3 Current Commutation Based on the Load Current . . . . . . . . . . . . 62

3.4 Current Commutation Based on the Voltage Across the Bi-directional Switches 66

3.5 Commutation of Current Between Bi-Directional Switches in the Residential Static VAR Compensator . . . . . . . . . . . . . . . . . . . . 69

4 Performance Analysis and Simulation Results of the Residential Static Var

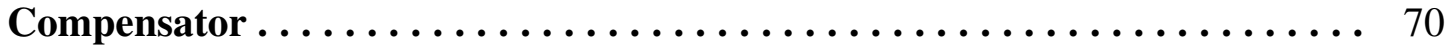

4.1 RSVC Open-Loop Design Using Matlab/Simulink . . . . . . . . . . . 70

4.1.1 Design of the Four-Step Current Commutation State Machine Based on the Sign of Voltage Across the Bi-Directional Switches . . . . . 71

4.1.2 Design of the Residential Static VAR Compensator (RSVC) . . . . 76

4.2 RSVC Open-Loop Simulation and Performance Analysis . . . . . . . . . 78

4.2.1 Voltage Waveforms $\ldots \ldots \ldots \ldots \ldots \ldots \ldots \ldots \ldots \ldots$

4.2.2 Current Waveforms $\ldots \ldots \ldots \ldots \ldots \ldots \ldots \ldots \ldots \ldots$

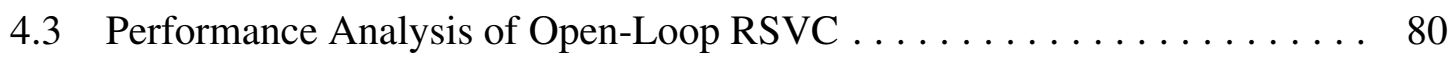

4.3.1 Output (load) Voltage Variation With Duty Cycle . . . . . . . . . 80

4.3.2 Reactive Power Variation With Duty Cycle . . . . . . . . . . . . . . 80

4.3.3 Input and Output Power Variation With Duty Cycle . . . . . . . . 82

4.4 RSVC Closed-Loop Design $\ldots \ldots \ldots \ldots \ldots \ldots \ldots \ldots \ldots \ldots \ldots$

4.4.1 Design of Voltage Regulation Control Loop . . . . . . . . . . . . . . 84

4.4.2 Integrator Windup in the PI controller . . . . . . . . . 86

4.5 Performance Analysis of Voltage Regulation Control Loop . . . . . . . . . . 88

4.5.1 Tracking the Reference Voltage $V_{r e f} \ldots \ldots \ldots \ldots \ldots$

4.5.2 Voltage Regulation Loop Performance With Variable Input Voltage 90

4.5.3 Load Power Demand Shaving in a Distribution Feeder . . . . . . . . 93 
4.6 Design of Power Regulation Control Loop . . . . . . . . . . . . . . . 95

4.7 Performance Analysis of Power Regulation Control Loop . . . . . . . . . . . . 99

4.7.1 Tracking the Power for a $2 \mathrm{~kW}$ Resistive Load . . . . . . . . . . . . . 99

4.7.2 Tracking the Power for a Resistive Heater Load . . . . . . . . . . . 100

4.7.3 Tracking the Power for a Resistive Heater Load with Variable Input

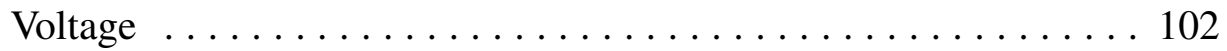

4.7.4 Tracking the Power for Loads with Negative CVR Factor . . . . . . 103

4.7.5 Performance of Power Regulation for Combined (Spot) Loads . . . 106

5 Design Implementation on SoC and Experimental Results . . . . . . . . 111

5.1 Open-loop SoC Design for RSVC Gating Signals . . . . . . . . . . . 111

5.2 Open-loop Experimental Testing Results $\ldots \ldots \ldots \ldots \ldots \ldots \ldots \ldots$

5.2 .1 Input and Output Voltage $\ldots \ldots \ldots \ldots \ldots \ldots \ldots \ldots \ldots \ldots$

5.2.2 Input (Source) Current . . . . . . . . . . . . . . 115

5.2 .3 Reactor Current . . . . . . . . . . . . . . . . 117

5.2.4 Current through the bi-directional switch . . . . . . . . . . 117

5.2.5 Output (Load) Voltage Variation with Duty Cycle . . . . . . . . . . . 119

5.2.6 Input and Output Power Variation with Duty Cycle . . . . . . . . . . 119

5.3 Closed-loop Design for RSVC $\ldots \ldots \ldots \ldots \ldots \ldots \ldots \ldots \ldots \ldots \ldots \ldots$

5.3 .1 SoC Implementation $\ldots \ldots \ldots \ldots \ldots \ldots \ldots \ldots \ldots \ldots \ldots \ldots \ldots$

5.4 Design of Voltage Regulation Control Loop . . . . . . . . . . . . . . . 123

5.4.1 Tracking the Reference Voltage $V_{r e f} \ldots \ldots \ldots \ldots \ldots \ldots . \ldots \ldots$

5.5 Design of Power Regulation Control Loop . . . . . . . . . . . . . . . 126

5.5.1 Tracking the Power for the Resistive Load . . . . . . . . . . . . . 126 


\section{Residential Peak Power Demand Shaving by Single-Phase Residential Static}

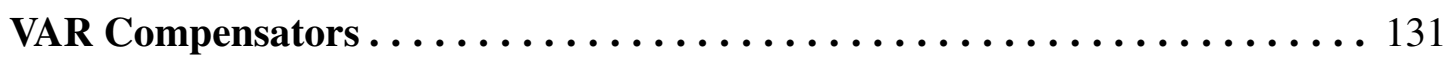

6.1 Peak Shaving on IEEE-13 Node Feeder using RSVCs . . . . . . . . . . 134

6.2 Peak Shaving on an Actual Feeder using RSVCs $\ldots \ldots \ldots \ldots \ldots \ldots$

6.2.1 Distribution Feeder Description . . . . . . . . . . . . . . . 137

6.2 .2 Load Model . . . . . . . . . . . . . . . . . . . . . . . . 138

6.3 Power and Voltage Profile for the Distribution Feeder . . . . . . . . . . . 139

6.4 A General Way of Approaching Peak Shaving Using CVR .......... 142

6.4.1 Changing the Substation LTC and Voltage Regulator Tap Settings . 142

6.5 Peak Shaving Using Single-Phase RSVC . . . . . . . . . . . . . . . 145

6.5.1 Simulation Method............................ 145

6.5.2 Simulation Results . . . . . . . . . . . . . . . . . . . 147

6.6 Cost Analysis and Performance benefits for Peak Shaving between RSVC

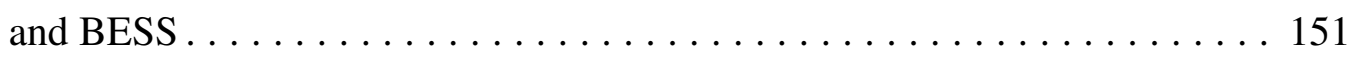

7 Conclusion And Future Work $\ldots \ldots \ldots \ldots \ldots \ldots \ldots \ldots \ldots \ldots \ldots \ldots \ldots \ldots \ldots \ldots \ldots \ldots$

7.1 Applications for Deploying the RSVC $\ldots \ldots \ldots \ldots \ldots \ldots \ldots \ldots \ldots$

7.1.1 Conservation of Power by Voltage Reduction (CVR) . . . . . . 156

7.1.2 Mitigation of Voltage Violation $\ldots \ldots \ldots \ldots \ldots \ldots \ldots \ldots \ldots$

7.1.3 Distribution Systems Assets Deferral . . . . . . . . . . . . 157

7.1.4 Peak Shaving During Peak-Demand Hours . . . . . . . . . . . . . 157

7.1.5 Power Factor Correction . . . . . . . . . . . . . . . . . . . . 157

7.1.6 Increasing the Hosting Capacity $\ldots \ldots \ldots \ldots \ldots \ldots \ldots \ldots \ldots \ldots$

$7.2 \quad$ Suggested Future Work $\ldots \ldots \ldots \ldots \ldots \ldots \ldots \ldots \ldots \ldots \ldots \ldots \ldots$

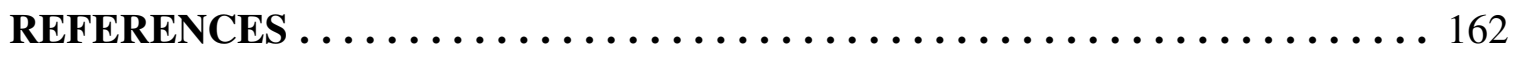


A Fourier Series Representation of a Pulse Function $\ldots \ldots \ldots \ldots \ldots \ldots \ldots$ 


\section{LIST OF TABLES}

1.1 CVR factor $C V R_{f}$ of different customer classes. . . . . . . . . 11

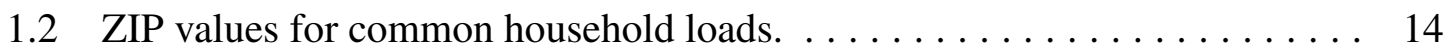

3.1 Comparison of different bi-directional switches topologies. . . . . . . . 60

3.2 Non-hazardous combinations for current commutation based on the direc-

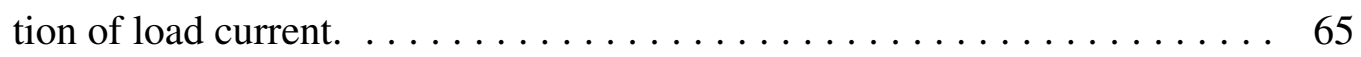

4.1 PI controller and saturation block parameters for the voltage regulation loop. 86

5.1 RSVC components used in the laboratory prototype. . . . . . . . . . 114

6.1 LTC and voltage regulator settings in the feeder without CVR. . . . . . 138

7.1 RSVC Suggested Power Electronics Components For Future Designs (labprototype). . . . . . . . . . . . . . . . . . . . . . . . . . . 159 


\section{LIST OF FIGURES}

1.1 Average annual growth rates of the world renewables supply from 1990 to

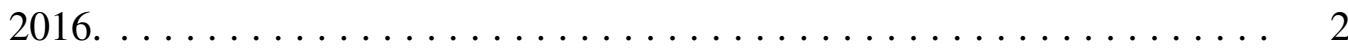

1.2 One-day wind power profile plotted with 1-minute average. . . . . . 3

1.3 Voltage variation due to change in solar irradiance for a solar plant in rural Idaho, USA from 6 AM till 1 PM plotted with 1-sec time interval. . . . . . . 4

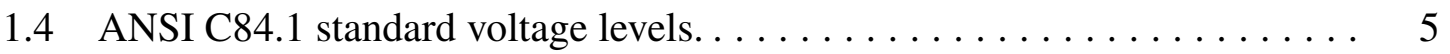

1.5 Voltage profile for a typical distribution feeder. . . . . . . . . . . 6

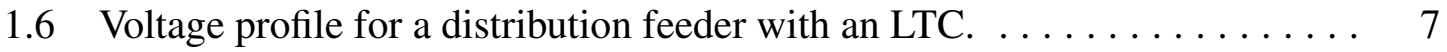

1.7 Voltage-dependent energy consumption of a Dell LCD. The plot shows the real and reactive power consumption, $P_{m}$ and $Q_{m}$ respectively, while operated between $100 \mathrm{~V}$ and $126 \mathrm{~V}$, whereas the red line indicates the voltage response curve using the least-square fitted ZIP values, $P_{e}$ and $Q_{e} \ldots \ldots \ldots 14$

1.8 An overview for the proposed control system for the closed-loop RSVC containing two regulation loops, namely the "Voltage Regulation Control Loop" and the "Power Regulation Control Loop". . . . . . . . . . . . . . . 16

2.1 Power transfer between two power sources. . . . . . . . . . . 20

2.2 Variable reactive power source connected in shunt with the load. . . . . . 22

2.3 Phasor diagram of an electrical grid during nominal operation. . . . . . . 22

2.4 Phasor diagram of an electrical grid during under-voltage operation with reactive shunt compensation. $\ldots \ldots \ldots \ldots \ldots \ldots \ldots \ldots \ldots \ldots \ldots \ldots$ 
2.5 Phasor diagram of an electrical grid during over-voltage operation without reactive shunt compensation. .................. 25

2.6 Radial AC system without reactive power compensation. . . . . . . . . 27

2.7 Radial AC system with shunt reactive power compensation. . . . . . . . 27

2.8 Radial AC system without reactive power compensation. . . . . . . . 28

2.9 Radial AC system with series reactive power compensation. . . . . . . . . 28

2.10 Different Configurations of Static VAR Compensator. . . . . . . . . . . . 30

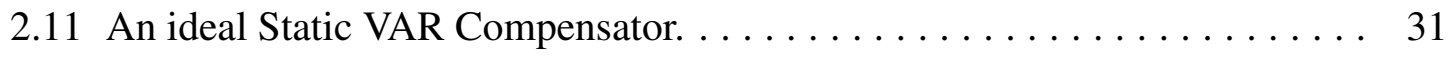

2.12 V/I characteristics of an SVC having a fixed capacitor and variable reactor. . 31

$2.13 \mathrm{~V} / \mathrm{I}$ characteristics of an SVC having a fixed capacitor and variable reactor with operating points for given power systems. . . . . . . . . 33

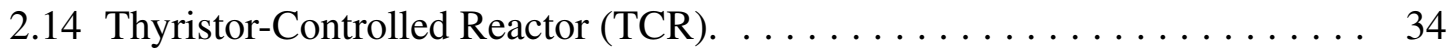

2.15 Voltage and current waveforms in a TCR for different thyristor gating angles. 35

2.16 Variation of the TCR susceptance $B_{T C R}$ with firing angle $\alpha \ldots \ldots \ldots \ldots$

2.17 Harmonics in the TCR current. . . . . . . . . . . . . . 40

2.18 Total Harmonic Distortion (THD) for the TCR current. . . . . . . . . . 41

2.19 A delta-connected three-phase TCR. . . . . . . . . . . . . 41

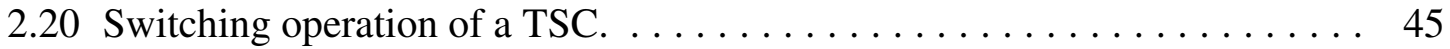

2.21 V/I characteristics of a multiple TSC with operating points for given power

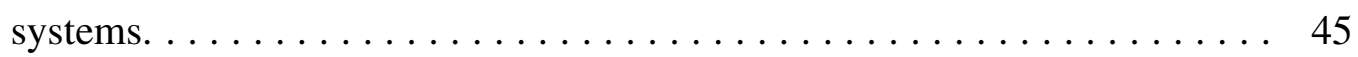

2.22 PWM-based switched reactor $\ldots \ldots \ldots \ldots \ldots \ldots \ldots \ldots \ldots$

2.23 The circuit for calculating the input current $i_{i n, n}$ harmonics. $\ldots \ldots \ldots \ldots 3$

2.24 Single-phase PWM-based switched reactor with fixed capacitor. . . . . . . . 54

2.25 The reactive power provided by the PWM-based switched reactor with a fixed capacitor compensator. . . . . . . . . . . . . . 56 


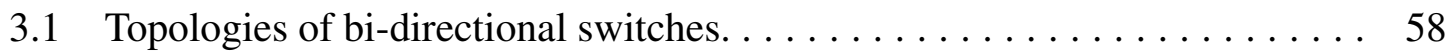

3.2 A simplified RSVC circuit to explain the problems with conventional switch-

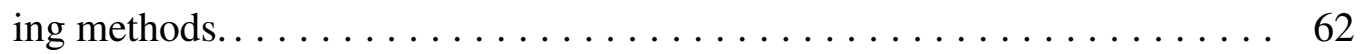

3.3 A circuit for understanding current commutation based on load current. . . 63

3.4 Four-step switching state machine for two bi-directional switches based on

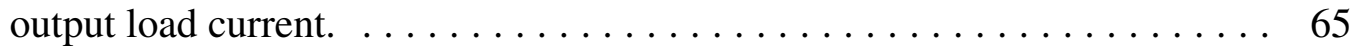

3.5 Positive voltage-based current commutation from SW1 to SW2 when the current direction is positive. $\ldots \ldots \ldots \ldots \ldots \ldots \ldots \ldots \ldots \ldots \ldots \ldots \ldots \ldots$

3.6 Positive voltage-based current commutation from SW1 to SW2 when the current direction is negative. . . . . . . . . . . . . 67

3.7 Four-step switching state machine for two bi-directional switches based on the voltage polarity across the bi-directional switches. . . . . . . . . 68

4.1 Circuit diagram of the Residential Static VAR Compensator. . . . . . . . . 71

4.2 Top-level block diagram for the state machine producing gating signals for the bi-directional switches. . . . . . . . . . . . . . . . . . . . . . . 72

4.3 A complete state machine for producing gating signals SW_1A, SW_1B, SW 2 A and SW_2B based on the output load voltage. The states (blocks) in the state machine represents the output of the state machine for each

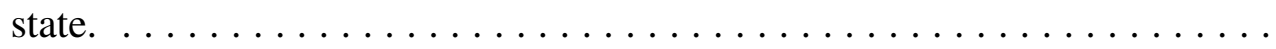

4.4 Output of the switch SW_1A from the state-machine for a complete cycle

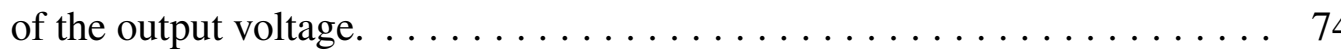

4.5 The gating signals for current commutation from SW1 to SW2 based on the amplitude of voltage when output voltage $V_{\text {out }}>0 \ldots \ldots \ldots \ldots$ 
4.6 The gating signals for current commutation from SW1 to SW2 based on the amplitude of voltage when output voltage $V_{\text {out }}<0 \ldots \ldots \ldots$

4.7 The RSVC Simulink model with a fixed capacitor and a switched inductor

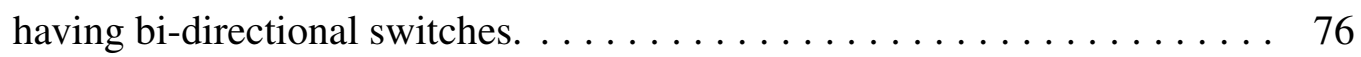

4.8 Bi-directional switches model in Simulink. . . . . . . . . . . . . . . . . 77

4.9 Input (source) and output (load) voltage waveforms at duty cycle $\mathrm{D}=0.5$. $\quad 78$

4.10 Input (source), output (load) and inductor current waveforms at duty cycle

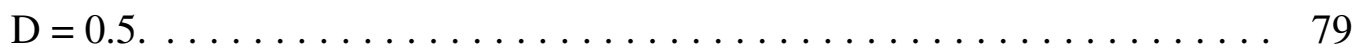

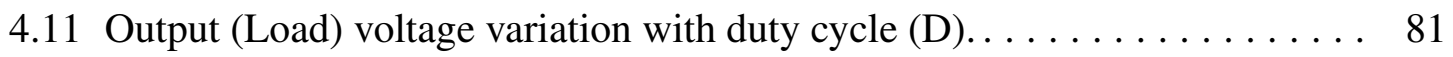

4.12 Net reactive power $\left(Q_{S V C}\right)$ variation with duty cycle $(\mathrm{D}), \ldots \ldots \ldots \ldots 2$

4.13 Input and output power variation with duty cycle (D) . . . . . . . 83

4.14 Block diagram of the feedback system for the voltage regulation loop of the Residential Static VAR Compensator. . . . . . . . . . . . . . . . 85

4.15 Block diagram for clamping method to avoid integrator windup. . . . . . . 87

4.16 Block diagram for back-calculation method to avoid integrator windup. . . 88

4.17 Voltages (input, output and reference) and reactive power change caused by the voltage regulation loop operation for an ideal input voltage. . . . . . . 89

4.18 Real powers (input and output) and duty cycle change caused by the voltage regulation loop operation for an ideal input voltage. . . . . . . . . . . . 90

4.19 Voltages (input, output and reference) and reactive power change caused by the voltage regulation loop operation for a variable input voltage. . . . . . 91

4.20 Real powers (input and output) and duty cycle change caused by the voltage regulation loop operation for a variable input voltage . . . . . . . . . . . 92 
4.21 Voltages (input, output and reference) and reactive power change caused by voltage regulation loop operation for a variable input voltage to achieve peak-demand shaving. . . . . . . . . . . . . . . . 94

4.22 Real powers (input and output) and duty cycle change caused by voltage regulation loop operation for a variable input voltage to achieve peak-

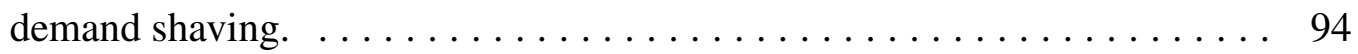

4.23 Complete Simulink block diagram of power regulation loop. . . . . . . 96

4.24 Block diagram of Power Loop P/O Decision subsystem. . . . . . . . . . . . 97

4.25 Block diagram of Accumulator subsystem. . . . . . . . . . . . . . 99

4.26 Voltages (input, output and reference) and real power (input and output) change caused by power regulation loop operation for an ideal input voltage with a $2 \mathrm{~kW}$ purely resistive load. . . . . . . . . . . . . . . 101

4.27 Net reactive power and duty cycle change caused by power regulation loop operation for an ideal input voltage with a $2 \mathrm{~kW}$ purely resistive load. . . . . 101

4.28 Voltages (input, output and reference) and real power (output) change caused by power regulation loop operation for an ideal input voltage with a resistive heater load.

4.29 Net reactive power and duty cycle change caused by power regulation loop operation for an ideal input voltage with a resistive heater load. . . . . . . 103

4.30 Voltages (input, output and reference) and real power (output) change caused by power regulation loop operation for a variable input voltage with a resistive heater load. . . . . . . . . . . . . . . . . . . . . . . . . . . . . . 104

4.31 Net reactive power and duty cycle change caused by power regulation loop operation for a variable input voltage with a resistive heater load. . . . . . . 104

4.32 An example of the load whose power increases as the voltage decreases... 105 
4.33 Voltages (input, output and reference) and real power (output) change caused by power regulation loop operation for an ideal input voltage with a load having negative CVR factor. . . . . . . . . . . . . . . . 107

4.34 Net reactive power and duty cycle change caused by power regulation loop operation for an ideal input voltage with a load having negative CVR factor. 107

4.35 Voltages (input, output and reference) and real power (output) change caused by power regulation loop operation for an ideal input voltage with a spot

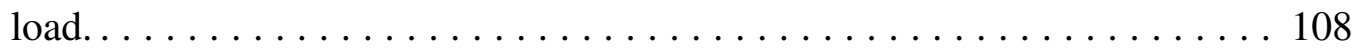

4.36 Net reactive power and duty cycle change caused by power regulation loop operation for an ideal input voltage with a spot load. . . . . . . . . . . . . 109

4.37 Change in power for individual loads as the voltage varies. . . . . . . . . . . 109

5.1 Complete block diagram for System-on-Chip implementations for generating the gating signals. . . . . . . . . . . . . . . 113

5.2 Laboratory prototype of Residential Static VAR Compensator (RSVC). . . 114

5.3 Experimental result for input (source) and output (load) voltage waveforms

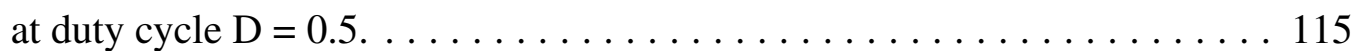

5.4 Experimental result for input (source) current at duty cycle $\mathrm{D}=0.5 \ldots \ldots 116$

5.5 Harmonic analysis of the input (source) current. . . . . . . . . . . . . 117

5.6 Experimental result for inductor current at different duty cycles. . . . . . . 118

5.7 Experimental result for current through top switch at different duty cycles. . 118

5.8 Experimental results for output (load) voltage variation with duty cycle (D). 120

5.9 Experimental results for input and output power variation with duty cycle (D). 
5.10 Complete block diagram for closed-loop RSVC control system design implemented in the Zedboard and dSPACE platform. . . . . . . . . . . . 122

5.11 Zynq-7000 SoC implementation of the state machine on processing logic (PL) and the timer interrupt on processing system (PS) for the development of the closed-loop RSVC on Zedboard. . . . . . . . . . . . . . . 123

5.12 Voltage regulation loop implemented in Simulink/dSPACE. . . . . . . . . 123

5.13 Plots of RSVC laboratory prototype with voltage regulation loop test results. 124

5.14 Power regulation loop implemented in Simulink/dSPACE. . . . . . . . . . 126

5.15 Complete control system for an RSVC laboratory prototype in Simulink/dSPACE.127

5.16 Plots of RSVC laboratory prototype with voltage regulation loop test results. 128

5.17 Plots of RSVC laboratory prototype with voltage regulation loop test results. 129

6.1 Single-line diagram for the IEEE-13 node test feeder. . . . . . . . . . . . . . 134

6.2 Voltage profile for the IEEE-13 node feeder with and without RSVCs. . . . 135

6.3 Peak power demand as function of the number of RSVCs. . . . . . . . 136

6.4 Feeder lines and voltage $(\mathrm{pu})$ for the distribution feeder. . . . . . . . . . 138

6.5 Annual power demand profile for the distribution feeder. . . . . . . . . . 140

6.6 Yearly voltage profile for the distribution feeder. . . . . . . . . . . . . . 141

6.7 Winter power demand profile for the distribution feeder. . . . . . . . . . . . 141

6.8 Maximum and minimum voltage across the distribution feeder at different tap settings for the substation LTC and in-line voltage regulator. . . . . . . 143

6.9 Winter power demand profile for the distribution feeder at different tap settings. . . . . . . . . . . . . . . . . . . . . . . . . 144

6.10 Flowchart for RSVC deployment strategy based on Worst-offender algorithm.146

6.11 RSVC placement in the distribution circuit. . . . . . . . . . . . 147 
6.12 Voltage profile of the distribution with and without the RSVC when the tap settings for LTC and in-line voltage regulators is set to $116 \mathrm{~V} \ldots \ldots \ldots \ldots . . .148$

6.13 Long-term RSVC-enhanced CVR power profile for the distribution feeder. . 149

6.14 Short-term RSVC-enhanced CVR power profile for the distribution feeder. . 150

6.15 Net reactive power provided by the RSVC devices during their operation

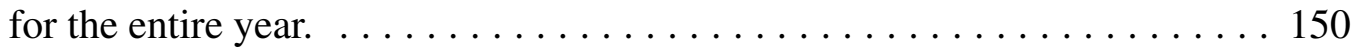

6.16 Transformer Deferral Calculation . . . . . . . . . . . . . . . 152

A.1 Periodic Pulse Function with an Amplitude of 1, time period of T and pulse

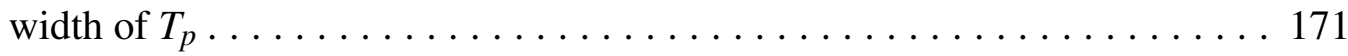




\section{LIST OF ABBREVIATIONS}

AMI - Advanced Metering Infrastructure

ANSI - American National Standards Institute

CVR - Conservation by Voltage Reduction

DERs - Distributed Energy Resources

EMF - Electromagnetic Force

EOL - End-of-Line

FACTS - Flexible AC Transmission Systems

FC-TCR - Fixed-Capacitor with Thyristor-Controlled Reactor

LTC - Load Tap Change

MinPPT - Minimum Power Point Tracker

MPPT - Maximum Power Point Tracker

P\&O - Perturb and Observe

PLL - Phase-Locked Loop

PV - PhotoVoltaic

PWM - Pulse Width Modulation

RSVC - Residential Static VAR Compensator

STATCOM - STATic synchronous COMpensator 
SVC - Static VAR Compensator

TCR - Thyristor-Controlled Reactor

THD - Total Harmonic Distortion

TSC - Thyristor-Switched Capacitor

TSR - Thyristor-Switched Reactor

VVO - Volt-VAR Optimization 


\section{CHAPTER 1}

\section{INTRODUCTION}

\subsection{Overview}

Electrical systems are undergoing a major transformation, from a simple one-way generation and distribution system to a complex network of multiple generating sources. The rapid increase in the use of distributed energy resources (DERs) since the turn of the century has made electrical generation and distribution systems into complicated systems. Renewable energy sources have shown a significant increase in the primary energy supply in the world from the period from 1990 to 2016 [1]. Since 1990, the electrical supply from renewable energy sources has grown at an annual rate of $2 \%$, which is higher than the growth rate of the total primary energy supply (TPES) of $1.7 \%$. The first two bars in Figure 1.1 show the average annual growth rate for the renewable energy supply from 1990 to 2016 and the average annual growth rate for the TPES over the same period. Moreover, the figure also shows an annual growth of the different renewables; with solar photovoltaic (PV) recording the maximum increase of $37.3 \%$, followed by wind at $23.6 \%$. This uptick in the generation of electricity from renewables is primarily due to government incentives and policies supporting green energy, investments from the private sectors, and the lower cost of production, especially PV panels. Research from Vibrant Clean Energy (VCE) shows that the United States has officially reached the "coal cost crossover point", where the rapidly declining cost of wind turbine and solar panel prices make operating three-quarters 
of all existing coal generation plants more expensive than building new local renewable energy [2]. It is estimated that by 2040 the share of electricity generation from renewables will increase from $25 \%$ today to around $40 \%$ worldwide [3].

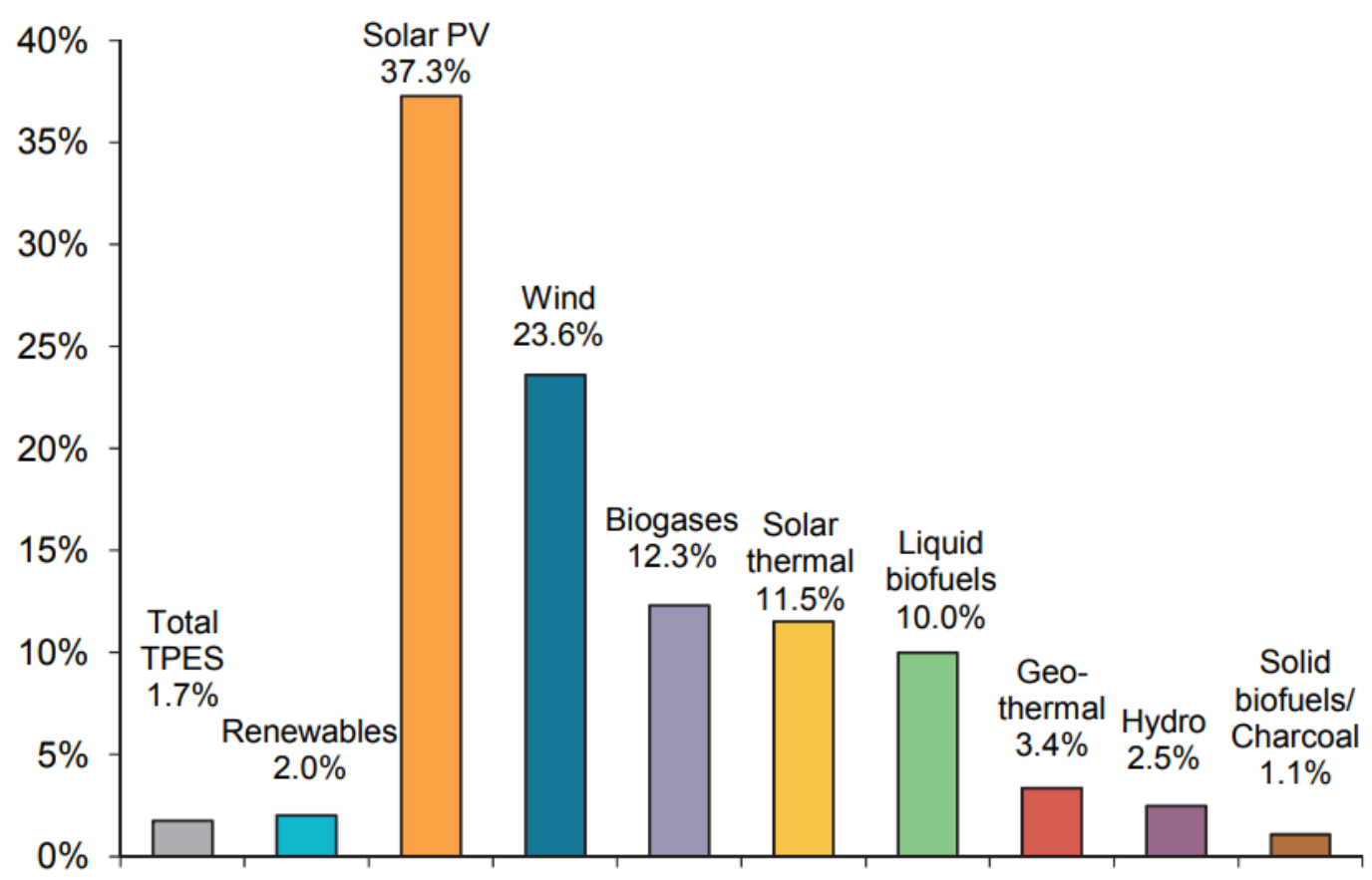

Figure 1.1: Average annual growth rates of the world renewables supply from 1990 to 2016 [1].

Renewable energy sources provide a great alternative for power generation when compared to traditional power generation from coal and oil which are associated with higher operational costs and carbon emissions in the environment. However, renewable energy sources, especially wind and solar, suffer from variability and unpredictability [4]. Figure 1.2 shows the variation in the wind power profile for a day, plotted with 1-minute average power, for the Electricity Reliability Council of Texas (ERCOT). The difference between daily maximum and minimum power is over 2,000 MW; the wind power started at about 3,000 MW at midnight and decreased to around 1,100 MW at $1 \mathrm{pm}$. The wind 
power increased again to about 3,300 MW at $5 \mathrm{pm}$. This graph points to the variability and unpredictability associated with power generation using wind as a renewable fuel. Similar behavior for variation in PV output power as a function of voltage can be observed in Figure 1.3. The voltage is measured at the point of common coupling of a solar plant connected with the electric grid in rural Idaho, USA. The voltage level fluctuates between $120 \mathrm{~V}$ to $124 \mathrm{~V}$ during the daytime due to the change in solar energy. Similar voltage variations due to fluctuations in solar irradiance can also be found in [5].

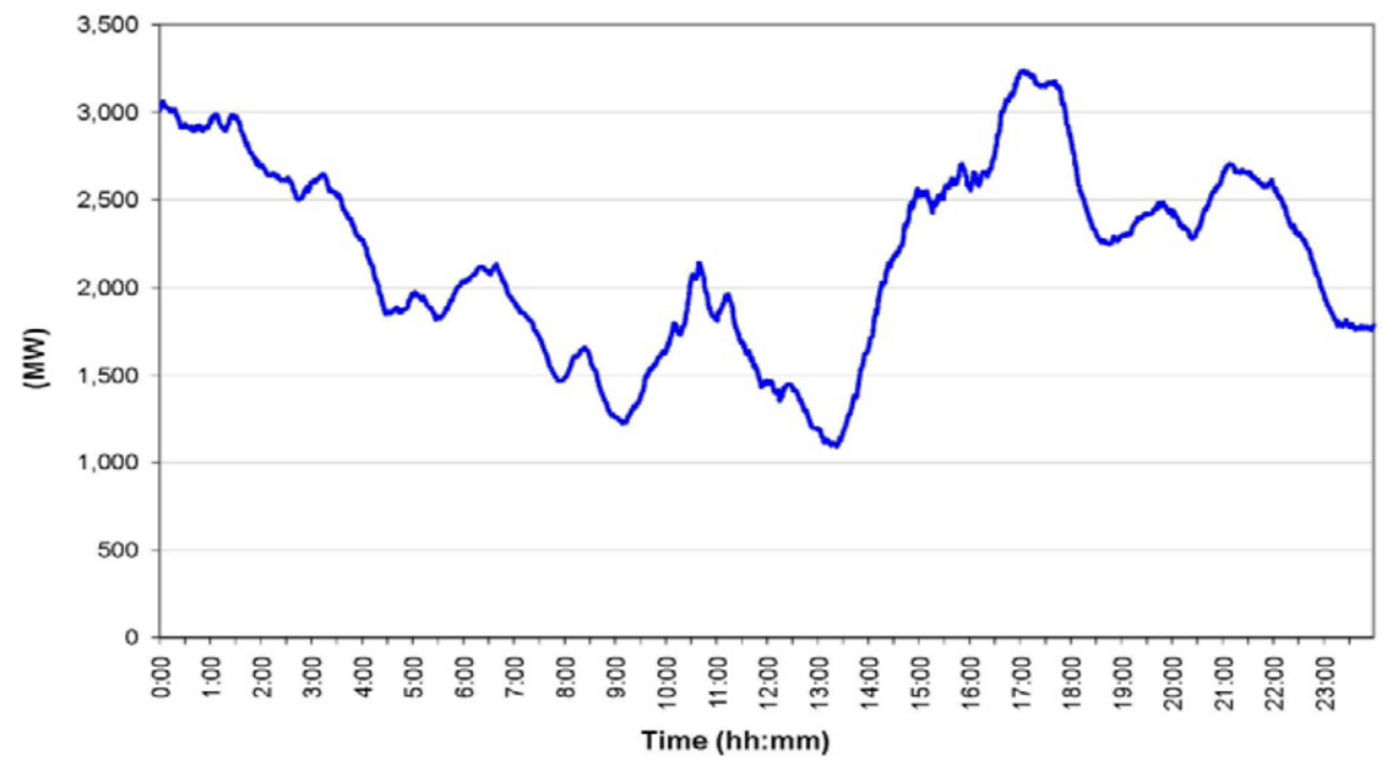

Figure 1.2: One-day wind power profile plotted with 1-minute average [4].

Therefore, higher penetration of renewables in the distribution system provides many challenges to the power system operators, mainly due to the unpredictability and variability of wind and solar power outputs. An accurate forecasting method for wind and solar power generation can help the power system operators to reduce the risk of the unreliability of the electricity supply $[5,6]$. But for any given instant, it becomes highly difficult to predict an accurate amount of power generation from renewable sources. Hence, it 


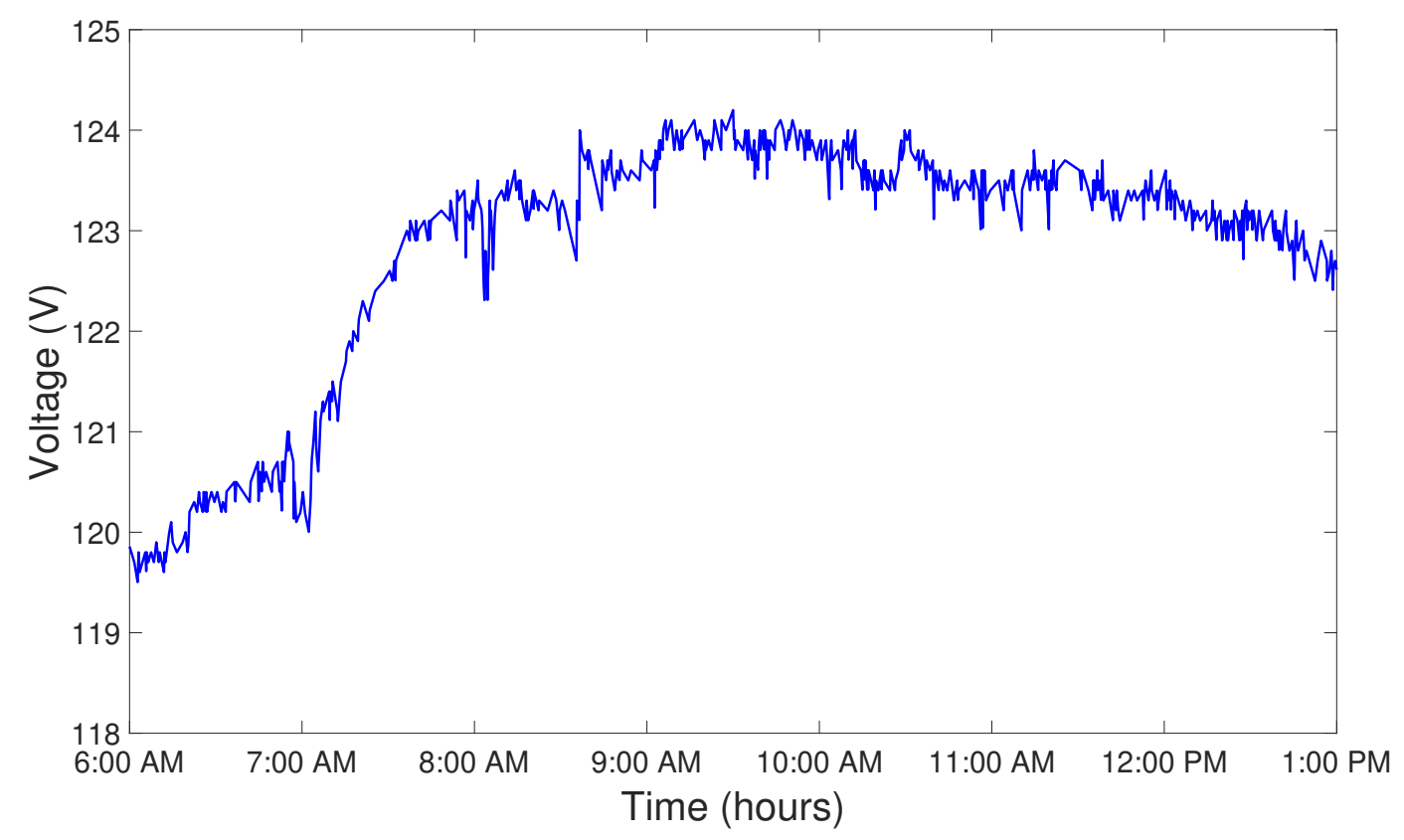

Figure 1.3: Voltage variation due to change in solar irradiance for a solar plant in rural Idaho, USA from 6 AM till 1 PM plotted with 1-sec time interval.

becomes imperative to research devices which can keep a consistent balance between power generation and demand at the consumer side of the power system.

For electric utilities, to guarantee a good quality service, loads should not be supplied with a voltage higher or lower than $5 \%$ of nominal voltage. The range of standard service voltages used in the United States is specified by the American National Standards Institute (ANSI) standard C84.1 - 2016 "Electrical Power Systems and Equipment - Voltage Ratings (60 Hertz)" as $120 \mathrm{~V}$ nominal, $114 \mathrm{~V}$ minimum (120 V minus 5\%) and $126 \mathrm{~V}$ maximum $(120 \mathrm{~V}$ plus 5\%). The voltage ranges in the ANSI standard for a nominal service voltage of $120 \mathrm{~V}$ is shown in Figure 1.4. In this figure, "Range A" shows the service voltage range of $\pm 5 \%$ of the nominal voltage of $120 \mathrm{~V}$ where as "Range B" specifies an out-of-normal service voltage range. The voltage levels mentioned in Range B are only acceptable for a brief period and should be infrequent. The electric utilities are mandated to operate 


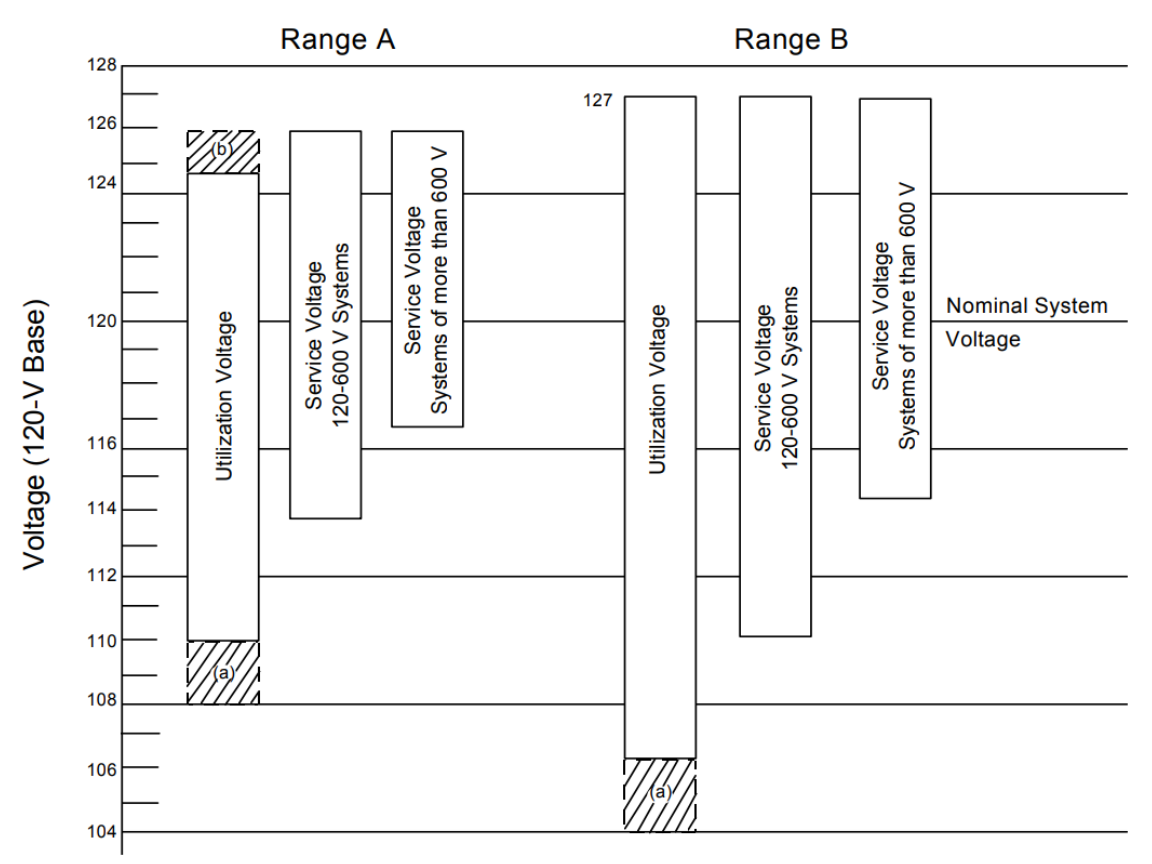

Figure 1.4: ANSI C84.1 standard voltage levels [7].

within a minimum voltage of $114 \mathrm{~V}$ at the secondary side of the distribution transformer to a maximum of $126 \mathrm{~V}$. The end-user equipment should be designed to operate at full performance within the service voltages limits of Range A (generally, the tolerance for the end-user equipment is from $110 \mathrm{~V}(120 \mathrm{~V}$ minus $10 \%)$ to $126 \mathrm{~V}(120 \mathrm{~V}$ plus $5.0 \%))$. Therefore, electric utility companies plan their distribution systems based on the voltage limits within Range A of the standard. However, with the variability associated with renewables and due to the variations in the nature of the loads connected to the distribution feeder throughout the year, it becomes challenging to maintain a voltage profile within the ANSI standards.

Moreover, a typical distribution feeder consists of several residential customers connected on the secondary side of the distribution transformer. In an ideal scenario, all loads connected along the feeder should get the same nominal service voltage, i.e., $120 \mathrm{~V}$. 


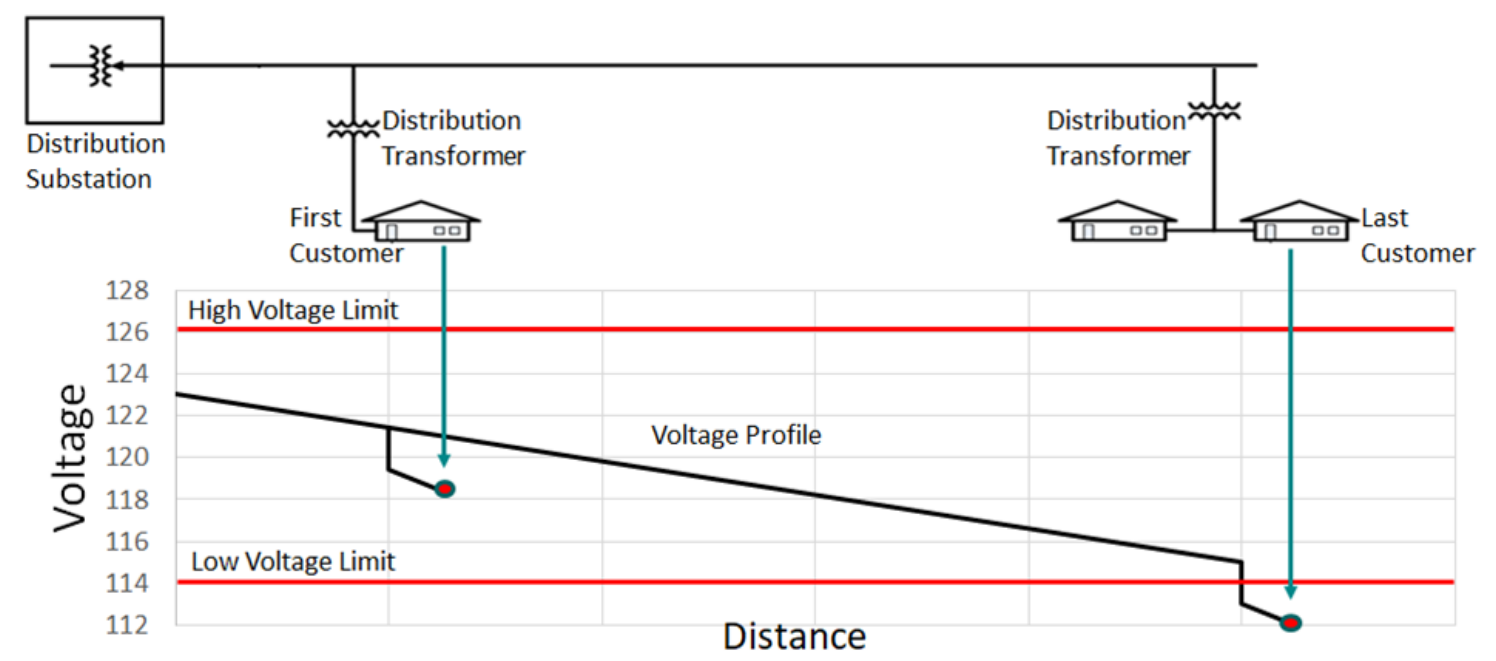

Figure 1.5: Voltage profile for a typical distribution feeder.

However, due to $I^{2} R$ power losses, the poor power factor of loads, and variations of loads throughout a day (or a season), customers experience less voltage than the voltage at the source of the distribution feeder. Generally, customers at the end of the distribution feeder face a significantly lower voltage than the customers closer to the feeder. A typical voltage profile for a distribution feeder is shown in Figure 1.5. It can be seen that the end-of-line voltage is below $114 \mathrm{~V}$, which is the minimum permissible voltage level according to the ANSI standard.

Electric utilities often employ passive voltage regulation methods to maintain the voltage within the ANSI standard. One typical approach is to use a Load Tap Change (LTC) transformer to regulate the voltage of the distribution feeder. Taps are connections on a distribution transformer winding that change the turn-ratio $N 1 / N 2$, where $N 1$ and $N 2$ are the numbers of turns on the primary and secondary side of a transformer respectively. Figure 1.6 shows the effect of an LTC on a feeder that is experiencing a low-voltage at the end-of-line. Other common approaches used by electric utilities to tackle the problem 


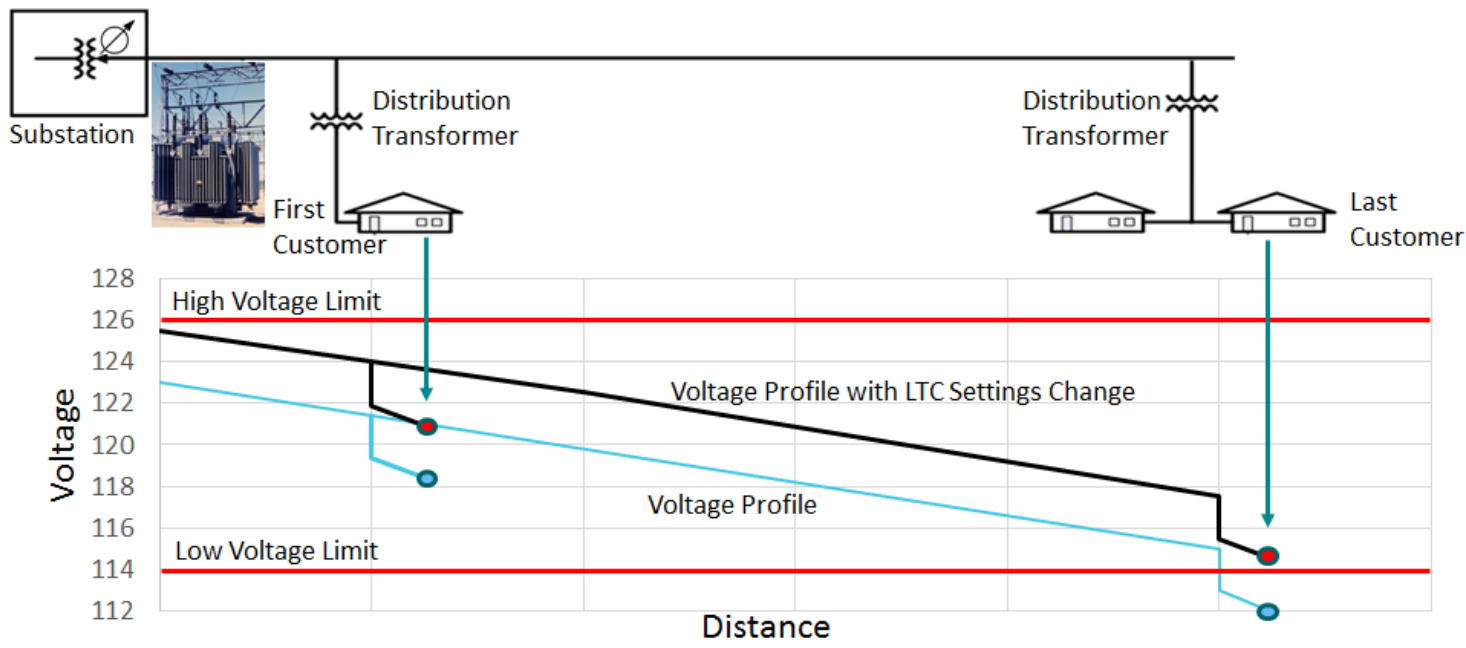

Figure 1.6: Voltage profile for a distribution feeder with an LTC.

of service voltage outside the ANSI standard include the use of capacitor banks, in-line voltage regulators, decreasing $I^{2} R$ power losses by increasing the conductor size, and transferring loads to another feeder.

Due to a recent push to incorporate DERs within the existing power system, the general characteristics of a feeder voltage as shown in Figure 1.5 undergoes a voltage profile that is dependent on renewable fuel, i.e., wind speed and solar irradiance. An efficient way to encounter the intermittency associated with renewables is to have devices distributed across the distribution feeder which can provide a balance between the generation of renewables and keeping the customer voltage within the ANSI standard. This dissertation proposes a solution that involves installing an individual device or devices which will regulate the voltage before each customers service. 


\subsection{Research Motivation}

Modern reactive power compensation techniques use Flexible AC Transmission Systems (FACTS) devices that provide dynamic reactive power to the power system. FACTS devices are high-speed power electronics devices that combine advanced control system techniques with the fast processing power of embedded microprocessors to respond to the reactive power needs of the power system $[8,9]$. Commonly-used FACTS devices are Static VAR Compensators (SVCs) and inverter-based STATic synchronous COMpensators (STATCOMs). For utilities, FACTS technology has become an essential tool to alleviate certain problems associated with the scheduling of reactive power and to get the most service from their transmission and distribution networks by enhancing grid reliability [10].

This research focuses on developing a FACTS device for regulating residential voltages. The dissertation introduces a novel idea of the Residential Static VAR Compensator (RSVC), an improved FACTS device, based on the traditional Static VAR Compensators, with the following observations:

- With the advancement in high-power transistor devices such as MOSFETs and IGBTs, the role of FACTS devices has become critical for an electric utility in distribution systems. RSVC devices can provide a real-time (active) reactive power compensation, therefore, facilitating the integration of renewables in the distribution system and regulating the voltage levels at the customer location.

- Electric utilities are required to become efficient and competitive by working to reduce costs. One such major cost is when a company buys energy from another utility in the market, i.e., when it cannot satisfy its peak-demand with its installed capacity. Therefore distribution companies, as well as residential customers, must pay a higher price per $\mathrm{kWh}$ during peak-demand hours. The goal of the proposed 
Residential Static VAR Compensator is to develop a device which helps to reduce power consumption during peak hours to save energy and costs by lowering customer voltage to a lower voltage level, commonly known as Conservation of power by Voltage Reduction (CVR), but still maintaining a relatively flat voltage profile across the distribution feeder.

- A localized approach to conservation of power by voltage regulation has the merit of adapting to the nature of the customer load, which may or may not decrease its energy consumption under a reduced voltage. This local approach is a radical departure from current CVR strategies, which have been in existence for over 30 years, but have not been widely adopted by electric utilities due to high costs and technical challenges.

Therefore, the development of RSVC will assist in the integration of DERs, provide a mechanism to effectively implement CVR across the distribution feeder and conform to the nature of individual load(s). The following sections will introduce CVR and its benefits from the studies published in the literature. A separate section will highlight the importance of understanding the nature of the loads and signify the importance of a localized CVR instead of conventional approach of implementing CVR.

\subsubsection{Conservation by Voltage Reduction (CVR)}

Conservation by Voltage Reduction (CVR) is the implementation of a distribution voltage strategy whereby all voltages are lowered to the minimum allowed by the equipment manufacturer. This is a consequence of the observation that many loads consume less power when they are fed with a voltage lower than nominal voltage. CVR works on the principle that customer appliances can be operated in the lower half of the ANSI C84.1 standard Range A, $114 \mathrm{~V}$ to $120 \mathrm{~V}$, without causing any harm to the appliances $[11,12]$. 
CVR effectiveness is measured by the CVR factor. The real power CVR factor is defined as the ratio of the percentage of the real power saved to the percentage of the voltage reduction used to achieve power saving. The CVR factor, $C V R_{P}$, can be represented in mathematical form as given in Equation (1.1),

$$
C V R_{P}=\frac{\Delta P \%}{\Delta V \%}
$$

where $\Delta P \%$ is the percentage of real power saved and $\Delta V \%$ is the percentage of voltage reduced. Electric utilities employ CVR for short-term demand response management as well as for long-term power reduction. For short-term CVR, the voltage is reduced for a short duration to reduce the peak-demand and for long-term, the voltage is reduced permanently to save power.

Electric utilities have long-known the advantages of distribution voltage reduction. In the 1970s, due to petroleum fuel shortages, electric utilities began investigating the effects of reducing the voltage at the customer level as a method for energy conservation. In 1973, American Electric Power (AEP) conducted a study to examine the impact of voltage reduction on the loads. Their test results showed significant savings by reducing the level of residential voltages. In the study conducted by AEP, the voltage levels were reduced by $5 \%$ for four hours. In one of the distribution feeders under consideration, a $5 \%$ voltage drop for 4 hours a day, from 9 AM to $1 \mathrm{PM}$, resulted in an immediate saving of $4 \%$ in the load power demand [13].

Since then electric power companies have constantly tested distribution voltage reduction and proven its impact. The paper [14] summarizes CVR factors from several CVR studies published in the literature. Table 1.1 shows CVR factors based on different 
customer classes.

Table 1.1: CVR factor $C V R_{f}$ of different customer class [14].

\begin{tabular}{|c|c|c|c|}
\hline References & Residential & Commercial & Industrial \\
\hline \hline California [15] & 0.76 & 0.99 & 0.41 \\
\hline BPA [16] & 0.77 & 0.99 & 0.41 \\
\hline AEP [12,17] & 0.61 & 0.89 & 0.35 \\
\hline CPUC [11] & 1.14 & 0.26 & N/A \\
\hline SCE [18] & 1.30 & 1.20 & 0.50 \\
\hline Snohomish [19] & $0.33-0.68$ & $0.89-1.10$ & N/A \\
\hline HQ [20] & $0.06-0.67$ & $0.80-0.97$ & 0.10 \\
\hline NEEA [21] & 0.63 & 0.37 & N/A \\
\hline Detroit [22] & $0.96-1.11$ & $0.75-0.80$ & $0.50-0.83$ \\
\hline
\end{tabular}

A general conclusion can be drawn by looking at the CVR factors in Table 1.1, that reducing voltage results in energy savings. However, before lowering the voltage, electric utilities must understand the behavior of the loads under reduced voltages. Closed-loop loads (loads with control mechanisms to regulate their operational performance when the voltage is reduced) operate longer to give the same service when the voltage is reduced. Typical closed-loop loads consist of loads with thermostat/thermal cycles such as electric water heaters, and regulated constant power loads such as furnaces and ovens.

Applying CVR can also reduce the core losses including eddy current losses and hysteresis losses of the distribution transformer [23]. On the other hand, $I^{2} R$ power losses in the transmission and distribution lines may increase due to constant-power loads since the current increases proportionally as a result of reduced voltage. However, this is not true for loads with constant-impedance and constant-current characteristics. Whereas lowering the voltage may increase line power losses, the decrease in power consumption is expected to be larger, so that the overall energy savings will be positive [20,24]. 
For electric utilities, CVR can become a tool for demand-side management. To summarize, the CVR benefits include a reduction in net power loss including $I^{2} R$ losses in the transmission lines; reduction in eddy currents and hysteresis losses in the transformers; peak-load management without buying power from other utilities; delay in critical assets upgrade such as substation transformer and less fuel consumption thus helping the environment.

\subsubsection{Traditional Methods for CVR Implementation}

Electric utilities often employ load tap change (LTC) transformers at the feeder source to implement CVR. As every distribution circuit has its load demand characteristics, it becomes difficult for electric utilities to impose a particular strategy for energy savings from one distribution feeder to another. When electric utilities apply CVR on a distribution feeder, the LTC transformer at the distribution substation decreases the feeder voltages. However, it becomes a challenge to keep the end-of-line voltage within an acceptable range. Due to voltage drops, utilities avoid reducing the voltage at the substation to the minimum permissible value and cannot get the maximum benefits from CVR at all points along the distribution network.

The paper [15] uses lower tap settings on LTC transformers to employ CVR. Their CVR strategy used switched shunt capacitors across the feeder to boost the voltage at lowvoltage locations. By placing capacitors at different locations, it is possible to attain a flat voltage profile, improve the power factor and reduce power losses. However, for solutions employing switched shunt capacitors to implement CVR, the placement of the capacitors becomes a challenging issue. Reference [25] discusses the optimal placement of capacitors in the power network. There is not much literature published for the optimal placement 
of capacitors for CVR purposes, however, one such reference [26] discusses the optimal capacitor placement for CVR purposes.

Closed-loop Volt-VAR Optimization (VVO) devices are more suitable for CVR application as they can determine the best voltage and VAR combination for a certain period and can adapt to dynamic changes in the loads. Specialized equipment and tools from Varentec [27], Gridco [28] and, Utilidata [29] are commercially available. Electric utilities have also employed Advanced Metering Infrastructure (AMI) meters-based VVO [30] and SCADA-based VVO [31] for CVR purposes.

\subsubsection{Minimum Power Point Tracker (MinPPT) / Optimal Operational Power Tracker}

CVR is an important tool for electric utilities to save energy, however the load composition has changed substantially by the turn of the 21 st century. With the increase in the number of power electronics devices in households, such as phone and laptop chargers, flat-screen televisions, liquid crystal displays (LCD’s), plasma displays, etc., the behavior of the load has changed as the voltage varies [32]. A general perception is that the appliances consume less energy at reduced voltage. This might not be true given the complex nature of modern loads.

In the literature, ZIP coefficients model is generally used to represent the steady-state load composition of various types of residential loads. A ZIP coefficients model accurately describes the steady-state behavior of modern loads under varying voltage conditions. The loads can generally be grouped into three categories depending on how their power demand varies with respect to the voltage at their terminal. For the constant power $(\mathrm{P})$ load, the power demand remains constant regardless of the voltage at the terminals of the load, for the constant current (I) load, the power demand is proportional to the load voltage and for the constant impedance $(\mathrm{Z})$ load, the power demand is proportional to the terminal voltage 
squared. In 2010, a study conducted by the Pacific Northwest National Laboratory (PNNL) reported the ratios for $\mathrm{Z}$, I, and $\mathrm{P}$ for several household loads [33]. Table 1.2 summarizes the ZIP values for seven common household loads.

Table 1.2: ZIP values for common household loads [33].

\begin{tabular}{|c|c|c|c|}
\hline Loads & $\mathrm{Z} \%$ & $\mathrm{I} \%$ & $\mathrm{P} \%$ \\
\hline \hline Incandescent Light Bulb (70W) & 57.11 & 42.57 & 0.32 \\
\hline Magnavox Television (Cathode Ray Tube) & 0.15 & 82.66 & 17.19 \\
\hline Oscillating Fan & 73.32 & 25.34 & 1.35 \\
\hline Liquid Crystal Display (Dell) & -40.70 & 46.29 & 94.41 \\
\hline Plasma TV (Sony) & -32.07 & 48.36 & 83.71 \\
\hline Liquid Crystal Display (Clarity TV) & 3.83 & 3.96 & 99.87 \\
\hline Compact Fluorescent Light (42W) & 48.67 & -37.52 & 88.84 \\
\hline
\end{tabular}
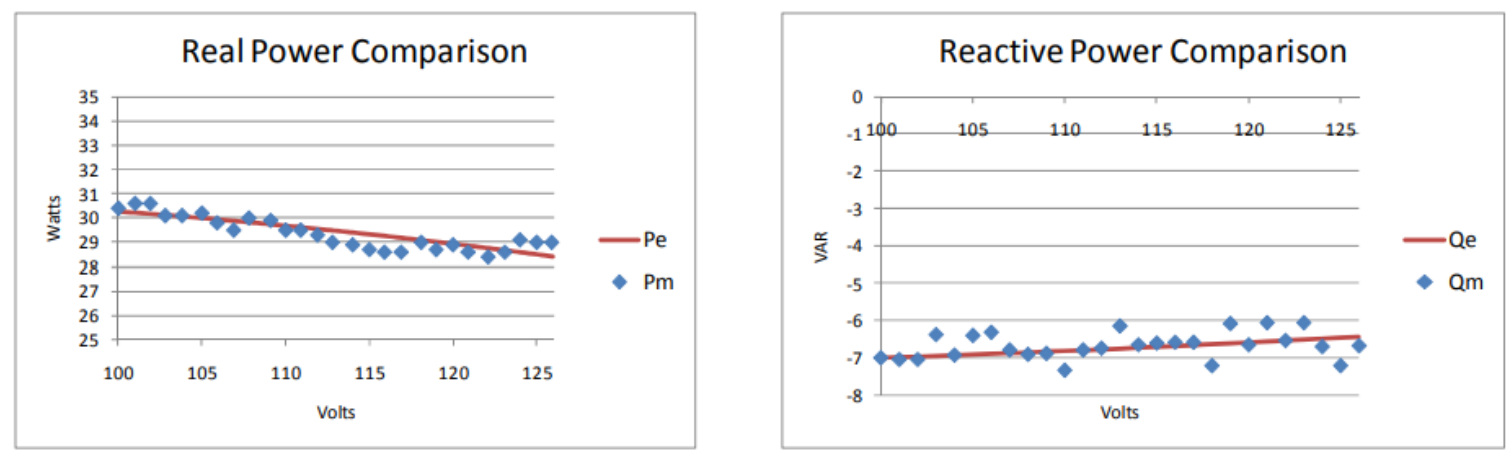

Figure 1.7: Voltage-dependent energy consumption of a Dell LCD. The plot shows the real and reactive power consumption, $P_{m}$ and $Q_{m}$ respectively, while operated between $100 \mathrm{~V}$ and $126 \mathrm{~V}$, whereas the red line indicates the voltage response curve using the least-square fitted ZIP values, $P_{e}$ and $Q_{e}$ [33].

The negative ZIP values in the table mean that the power generated from one component in the load is consumed by another component. The overall percentage sum for the three elements is always equal to $100 \%$. It can be inferred intuitively that loads with a higher P coefficient results in a negative CVR factor according to Equation (1.1), i.e., the energy 
consumption increases by reducing the voltage. This can be seen in Figure 1.7, which shows the voltage profile for a Dell LCD. It can be seen that the power consumption increases as the voltage is reduced.

The reference [34] lists ZIP values for more residential loads, as well as for four different commercial customers that included a supermarket, a restaurant, a laundromat and an optical store. Among the commercial loads, the supermarket load had the one with the maximum $\mathrm{P}$ (constant power) element in the ZIP value because of the dominance of heating, ventilation and air conditioning (HVAC) systems. Therefore, for feeders and distribution circuits where CVR does not yield energy savings, an autonomous localized voltage tracking strategy should be adopted. Using such a strategy will ensure that the minimum power-point is tracked with modern loads that employ an abundance of switching power supplies and spot loads that contain a higher percentage of HVAC loads regardless of the voltage level.

\subsection{Proposed Solution - Residential Static VAR Compensator (RSVC)}

This research focuses on developing a solution based on the concept of a Residential Static VAR Compensator for regulating residential voltages, especially during peak-demand hours when the benefits coincide best with the interests of customers and those of electric companies [35]. These RSVC devices can be an additional tool for smart demand-side management. By remotely controlling the RSVC, a utility can apply CVR at specified individual locations during specified periods. The goal of the development of the RSVC is to enhance the CVR by boosting the voltage at the circuit low points to the allowable minimum, such that the source of the feeder voltage can be reduced. By reducing the feeder source voltage, the entire distribution network voltage can take part in conservation by voltage reduction 
(CVR). As stated earlier, CVR relies on the idea that a percentage of the load on the distribution feeder will be of constant impedance. With a constant impedance load, if the voltage decreases, the power decreases. By decreasing the feeder power, the overall energy conservation will be improved. Therefore, the RSVC control system plays an essential role in controlling the reactive power provided by the device to the regulation bus.

To incorporate modern loads with switching power supplies and composite loads where a larger percentage is made of constant power loads, a minimum/optimal power point tracking is proposed in this dissertation. Hence, the overall control system for an RSVC will consist of two regulation loops. The objective of the first control loop will be to implement an inner voltage regulation loop for an RSVC and the goal of the second control loop will be to implement the outer power regulation loop for an RSVC. Figure 1.8 shows a simplified control systems design for an RSVC system. The voltage control loop acts as a faster control loop than the outer power minimization/optimization loop.

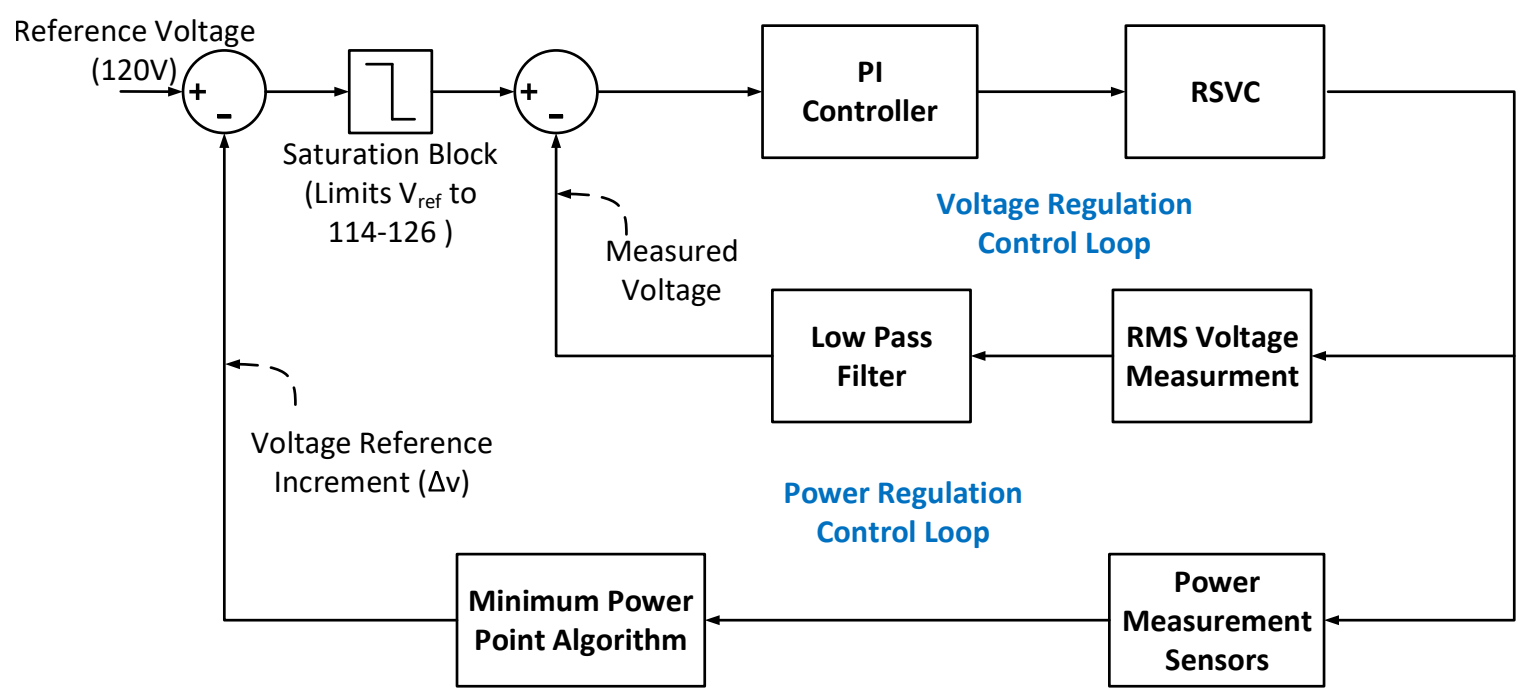

Figure 1.8: An overview for the proposed control system for the closed-loop RSVC containing two regulation loops, namely the "Voltage Regulation Control Loop" and the "Power Regulation Control Loop". 
The objectives intended to be achieved by the RSVC are given below:

- The device can regulate the customer load voltage at the desired reference level regardless of variable and fluctuating power injected by distributed energy resources.

- The device can minimize customer power consumption by tracking the optimal reference voltage.

- The device does not generate substantial harmonics which might require filtering and incur more development cost.

- The device will have a relatively lower cost compared to existing competitive devices on the market, even though they operate using the same principles.

\subsection{Organization of the Dissertation}

This dissertation is organized into seven chapters. Chapter 2 provides an overview of the principles of reactive power compensation. Traditional VAR compensators are reviewed, with emphasis on a Thyristor-Controlled Reactor (TCR). A novel approach for reactor switching based on a PWM technique is described in this chapter. The drawbacks of TCR and advantages for a PWM-based reactor switching over a traditional thyristor angle firing switching are also discussed in the chapter.

Chapter 3 describes the basic issues of the current commutation from one phase to other involving bi-directional switches. Different topologies for bi-directional switches are reviewed and the possibilities for current commutation through the bi-directional switches based on the sign of the voltage across the bi-directional switches or the direction of the output current is also discussed. For the single-phase RSVC prototype, the preference 
of the current commutation through the bi-directional switches based on the voltage sign across the switches is explained.

Chapter 4 presents a detailed guide for simulating open-loop and closed-loop RSVC in Simulink. The chapter also shows the performance of the open-loop and closed-loop RSVC design. Simulation results showing the performance of the voltage regulation loop to track the reference voltage with normal and unstable input voltage are presented in this chapter. The chapter also shows the performance of the power regulation loop involving loads with positive and negative CVR factors and adjusting the reference voltage so that minimum power is utilized.

Chapter 5 presents the experimental setup for testing the laboratory prototype of the RSVC system. The performance of the open-loop RSVC laboratory prototype is analyzed and discussed on the basis of the experimental results. Moreover, the experimental results from the closed-loop RSVC testing and their performance with a resistive load are also presented.

Chapter 6 includes a case study for the deployment of RSVC in an actual distribution feeder to attain RSVC-enhanced CVR. It is shown that the peak shaving on the distribution feeder can be performed by deploying RSVC devices, which can provide an alternative to batteries commonly used for peak shaving and load demand management.

Chapter 7 provides a brief introduction to the literature that has been published on RSVC. The literature includes applications of the RSVC which highlights the benefits of RSVC over existing-technologies. The chapter provides some recommendations for improving the RSVC prototype and also discusses the potential path to market for the RSVC devices. 


\section{CHAPTER 2}

\section{OPERATING PRINCIPLES OF RESIDENTIAL STATIC VAR COMPENSATORS}

A Residential Static VAR Compensator (RSVC) is an improved smart-grid device which aims to solve power quality issues in distribution systems and residential feeders. This chapter focuses on the operating principles of the RSVC and its interaction with the distribution and residential feeders based on understanding the power transfer between two single-phase sources in an AC power system. A brief review of the classical Static VAR Compensator (SVC) is also provided. The fundamental operating principle of the RSVC is to provide reactive power compensation with a superior performance than that of a classical SVC. The reactive power capabilities of the RSVC are also formulated and verified.

\subsection{Fundamental Principles of AC Power Flow}

In order to understand the operating principle of an RSVC, it is important to grasp the concept of AC power flow when there are two active energy sources in the system. Consider a power system as shown in Figure 2.1 with two energy sources labeled as $E_{G}$ and $E_{R}$. The energy source $E_{G}$ represents the power source (distribution/residential feeder) at the sending end and $E_{R}$ represents the power source (say a shunt capacitor or an RSVC) at the receiving end. The leakage reactance between the two sources is represented by an inductor of impedance $X$. 


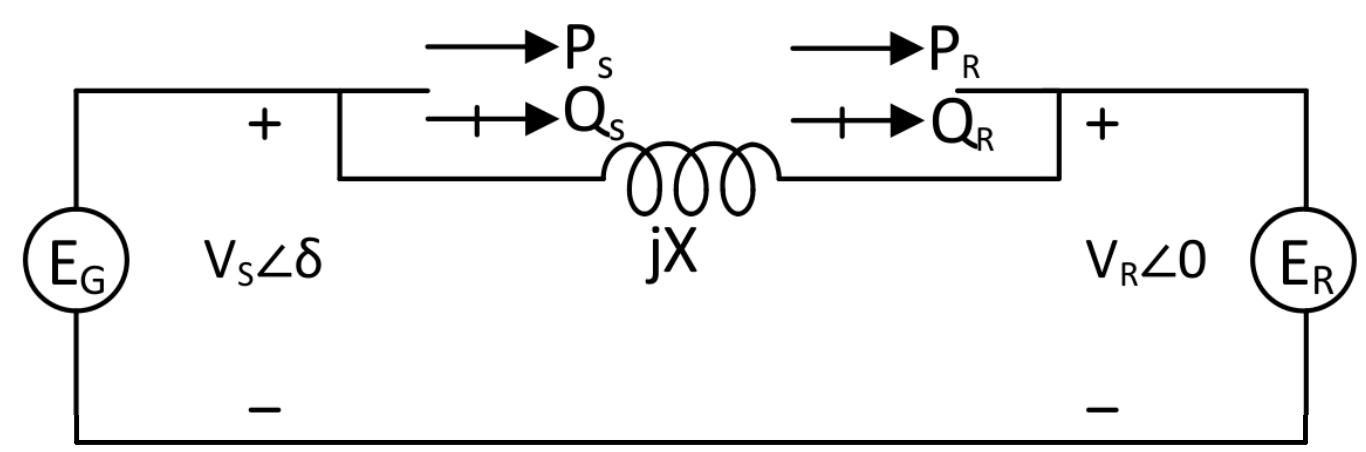

Figure 2.1: Power transfer between two power sources.

The complex powers at the sending and receiving ends of the power system are,

$$
\begin{aligned}
& \overline{S_{S}}=P_{S}+j Q_{S}=\frac{V_{S}}{X}\left(V_{R} \sin \delta-j V_{R} \cos \delta+j V_{S}\right) \\
& \overline{S_{R}}=P_{R}+j Q_{R}=\frac{V_{R}}{X}\left(V_{S} \sin \delta+j V_{S} \cos \delta-j V_{R}\right)
\end{aligned}
$$

In the distribution system, the difference between the angle in the sending end voltage and receiving end voltage, power angle $\delta$, is approximately equal to 0 . It can be approximated that there there is no real power transfer between the two power sources since the $\delta=0$. Equations (2.1) and (2.2) can be reduced to,

$$
\begin{gathered}
P_{S}=P_{R}=0 \\
\overline{S_{S}}=j Q_{S}=j \frac{V_{S}}{X}\left(V_{S}-V_{R}\right) \\
\overline{S_{R}}=j Q_{R}=j \frac{V_{R}}{X}\left(V_{S}-V_{R}\right)
\end{gathered}
$$

In this special case, it is seen that the flow of reactive power solely depends on voltage 
levels. From Equations (2.4) and (2.5), in the case where the sending end voltage is greater than the receiving end voltage, i.e., $V_{S}>V_{R}$, then both $Q_{S}$ and $Q_{R}$ are positive and $Q_{S}>Q_{R}$. This means that the reactive power flows from the sending end to the receiving end of the line. This case happens when a lagging or inductive current is flowing through the impedance $X$, therefore, resulting in a lower receiving end voltage. Similarly, when the sending end voltage is less than the receiving end voltage, i.e., $V_{S}<V_{R}$, both $Q_{S}$ and $Q_{R}$ are negative and $Q_{S}<Q_{R}$. This implies that the reactive power flows from the receiving end to sending end of the line. In this case, a leading or capacitive current is flowing through the impedance, which results in a higher receiving end voltage.

The change of the terminal voltages, $\Delta V=V_{S}-V_{R}$, depends on the reactive power. Equation (2.5) can be rewritten as,

$$
\Delta V=V_{S}-V_{R}=\frac{X Q_{R}}{V_{R}}
$$

where $V_{S}, V_{R}$ and $Q_{R}$ respectively show the sending end voltage, receiving end voltage and the reactive power drawn at the receiving end of the power system. If $\Delta V$ is positive, then the difference in the terminal voltages can be minimized by decreasing $Q_{R}$, which increases $V_{R}$. This mode of operation is called a capacitive mode. If $\Delta V$ is negative, then $Q_{R}$ is increased to minimize $\Delta V$, which decreases $V_{R}$. This mode of operation is called an inductive mode.

\subsection{Effect of Shunt Reactive Power Compensation}

Consider a power system as shown in Figure 2.2. The power system is similar to the power system shown in Figure 2.1 except there is a switch connected in series with energy source $E$, which is capable of providing variable reactive power. The power system consists of 
an energy source at the sending end $E_{G}$ with the voltage given by $V_{S}$. This energy source is connected with a load, $L$, via a transmission line having line reactance given by $X$. The output voltage at the load $L$ is given by $V_{R}$.

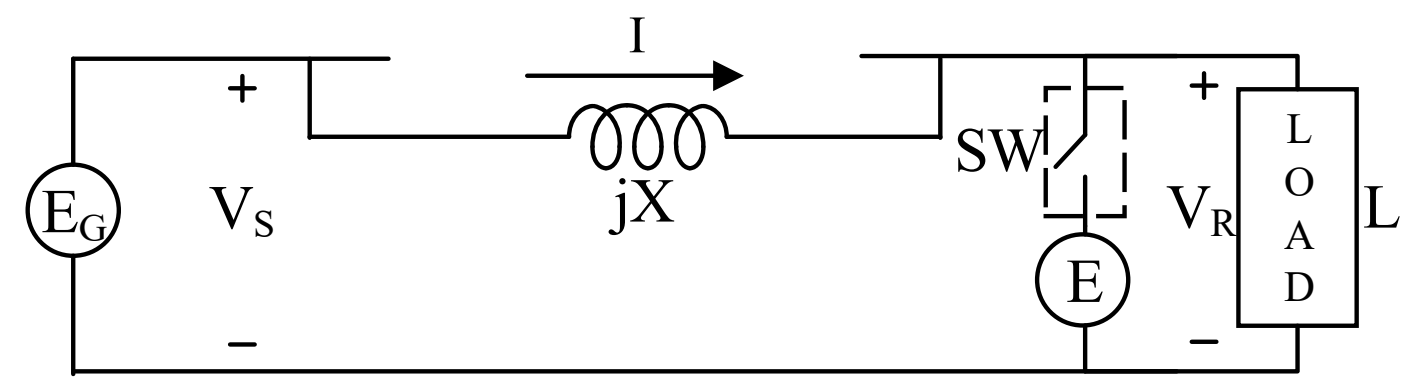

Figure 2.2: Variable reactive power source connected in shunt with the load.

For the reference case, consider that the variable reactive power source, $E$, is turned off, i.e., the switch $S W$ is open. Therefore, the vector sum of the input voltage is given as,

$$
\overrightarrow{V_{S}}=\overrightarrow{V_{R}}+j X \vec{I}
$$

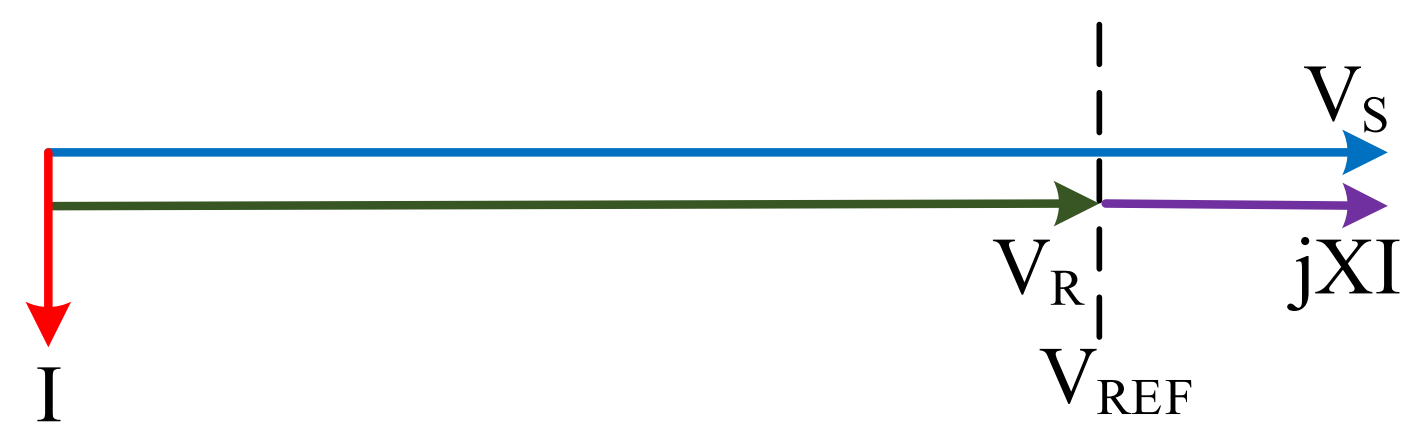

Figure 2.3: Phasor diagram of an electrical grid during nominal operation.

Figure 2.3 shows the phasor diagram of the power system without any contribution from $E$. The phasor diagram shows the normal operating conditions of the power system. 
During this condition, the load voltage at the customer in the distribution feeder is at the required reference level $V_{R E F}$.

To understand the effect of the shunt reactive power compensation using $E$, let's consider the following two modes:

1. When the voltage at the receiving end is less than the reference voltage, i.e., $V_{R}<$ $V_{R E F}$. In this case, the variable reactive power source works in the capacitive mode to boost the load voltage. For example, this case is typical for customers at the end of the distribution feeder;

2. When the load voltage at the receiving end is greater than the required reference voltage, i.e., $V_{R}>V_{R E F}$. In this case, the variable reactive power source suppresses the load voltage and works in the inductive mode. For example, this case arises when the electric utility implements CVR and the voltage at the feeder source needs to be dropped lower so that the entire feeder can participate in the CVR.

In the capacitive mode, the shunt energy source should inject reactive power to boost the load voltage. In other words, when switch $S W$ is connected to the power system $E$ should behave as a capacitor. The reactive power injected will flow to the load, reducing the amount of reactive power supplied by the source, therefore decreasing the voltage drop across the transmission line and increasing the load voltage. In terms of the terminal voltages, without the reactive power source $\Delta V=V_{S}-V_{R}$ is positive. The $\Delta V$ can be minimized by increasing the $V_{R}$. This can be achieved by reducing the reactive power flow in the transmission line by providing the reactive power the load consumes locally.

Figure 2.4 shows the phasor diagram of the system with the shunt reactive energy source. Without the reactive power source, the input current $I$ lags the input voltage $V_{S}$ therefore creating an under-voltage scenario at the receiving end of the feeder relative to 
the new reference voltage $V_{R E F, 1}$. When the reactive energy source is added to the power system, it acts as the capacitor, thus making the input current $I_{1}$ less inductive. As a result, the receiving end voltage is higher than the initial level.

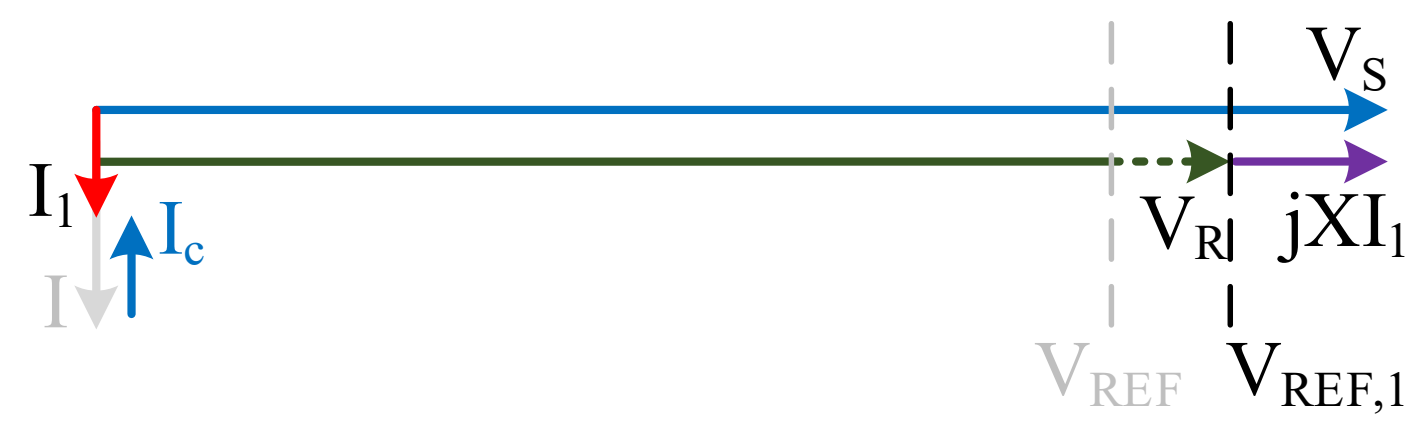

Figure 2.4: Phasor diagram of an electrical grid during under-voltage operation with reactive shunt compensation.

In the inductive mode, the shunt energy source should absorb reactive power to decrease the load voltage. In other words, $E$ should behave as an inductor so as to make the current flowing through the transmission line more inductive. The phasor diagrams of the power system with the shunt reactive energy source is shown in Figure 2.5. Without the reactive power source, the input current $I$ lags the input voltage $V_{S}$. When the reactive energy source is added to the power system (for the over-voltage operation), it acts as an inductor, thus making the current $I_{2}$ more inductive and therefore making the receiving end voltage lower than the initial level.

In the phasor diagrams shown in this section, the sending end voltage $V_{S}$ is kept constant whereas the receiving end voltage $V_{R}$ is varied according to the reference voltage. The phasor diagrams show that the receiving end voltage can be adjusted with the help of shunt reactive energy source $E$, therefore enabling the electric utilities to control the voltages at different points across the distribution feeders. 


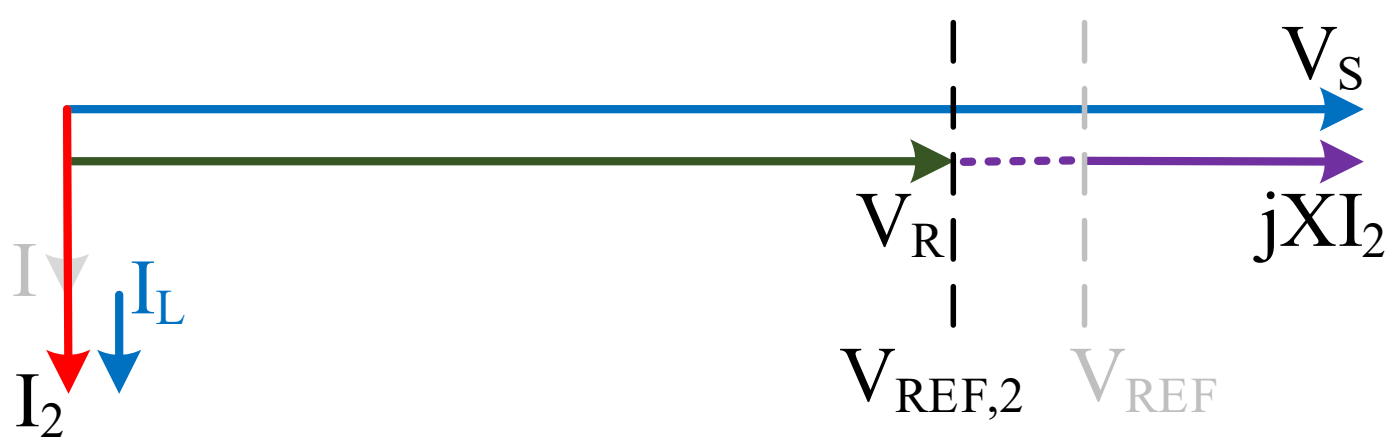

Figure 2.5: Phasor diagram of an electrical grid during over-voltage operation without reactive shunt compensation.

\subsection{Reactive Power Compensation Techniques}

The successful and efficient operation of a power system depends on the control of the voltages at the terminals of the equipment connected to the distribution feeders. The problem of maintaining the voltages at the customer's location within the required limits specified by the ANSI C84.1 standard is complicated. A distribution feeder supplies power to a vast number of loads. The composition of the load changes depending on the day, the season and the weather. However, the overall behavior of the loads is such that they absorb reactive power. Therefore, as the load varies, the reactive power requirements of the distribution feeder also vary. Moreover, with the growing number of distributed renewable generations, the power supplied by the generating units is not constant. Hence, additional means are usually required to control the voltage throughout the distribution feeder.

The generating units at the source of the transmission or distribution line provide the primary voltage across the power system. Electrical utilities are mandated to maintain voltage levels within a specified range at the residential level. Due to load variations and fluctuations in renewable generation, utilities rely on the flow of reactive power to control the voltage at the residential level. The devices that are used for this purpose can 
be classified as follows:

1. Reactive power sources and sinks, e.g., shunt capacitors, shunt reactors, synchronous condensers and Static VAR Compensators (SVCs).

2. Line reactance compensators, e.g., series capacitors.

3. Regulating transformers, such as tap-changing transformers and in-line voltage regulators.

Shunt capacitors provide reactive power at the point of connection and help boost local voltages. In the distribution system, shunt capacitors are extensively used to provide power factor correction. Most loads have a lagging power factor, i.e., they absorb reactive power. In some cases, shunt capacitors are deployed alongside local loads having lagging power factors rather than wheeling the reactive power from remote sources. The power system is compensated by providing a leading current to reduce the lagging current caused by an AC source. Figure 2.6 represents the phasor diagram of an AC system without shunt reactive compensation and Figure 2.7 shows the effect of shunt compensation in the overall AC system.

Another example of a shunt reactive power source/sink is synchronous condenser. A synchronous condenser is connected in shunt to provide variable reactive power to the electric grid. A synchronous condenser is a synchronous machine which is running without a primary mover or a mechanical load. The reactive power supplied by a synchronous condenser depends on the DC field excitation. For a leading power factor, a synchronous machine is overexcited, which produces a back electromagnetic force (EMF) greater than the applied voltage. Similarly, an underexcited synchronous machine produces a lagging power factor. 


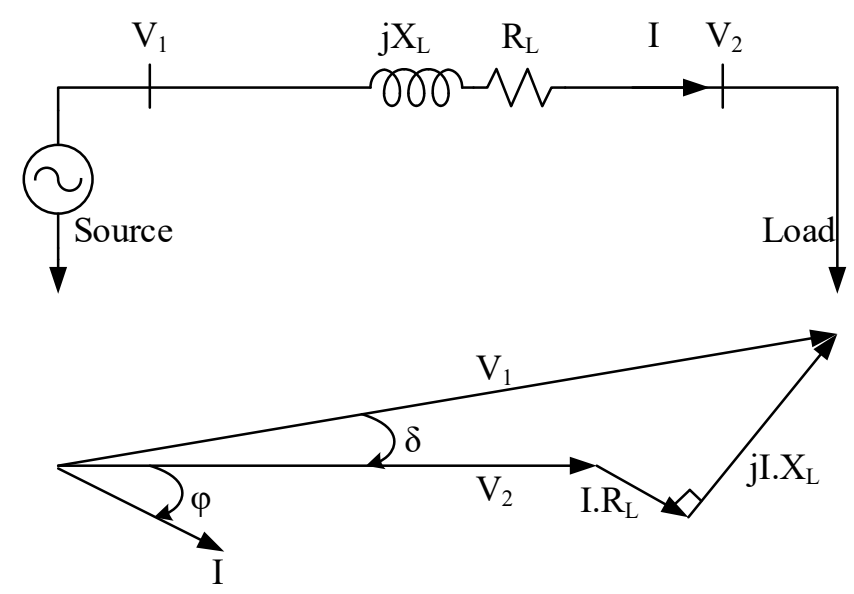

Figure 2.6: Radial AC system without reactive power compensation.

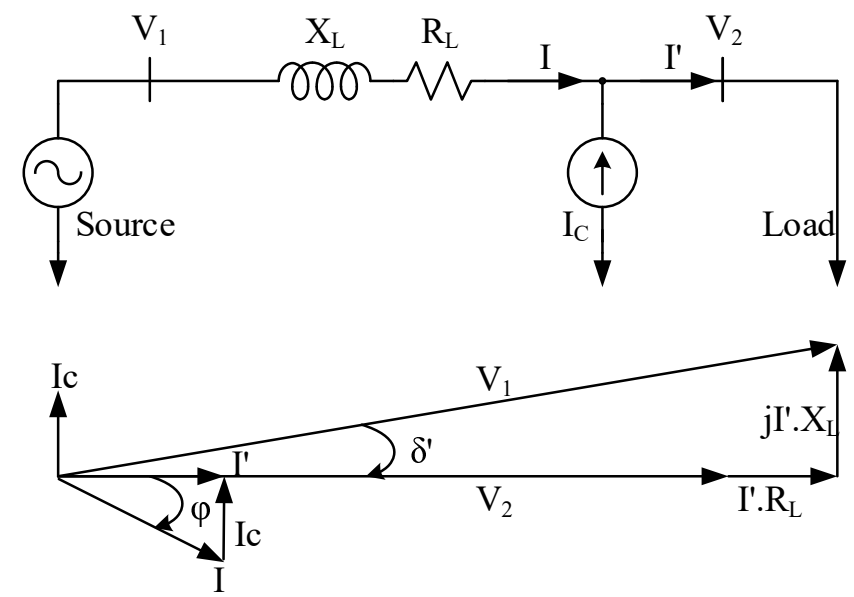

Figure 2.7: Radial AC system with shunt reactive power compensation.

Line compensation (or series compensators) involves decreasing the inductive reactance of power lines by installing series capacitors. This helps to reduce the effective reactive power loss $I^{2} X$ between the busses, decrease the voltage drop and increase the real power that can be transmitted. Figure 2.8 represents the phasor diagram of an AC system without series reactive compensation and Figure 2.9 shows the effect of series compensation in the overall AC system. A decrease in the line reactance not only reduces the voltage drop in the lines but also improves the voltage at the receiving end. 


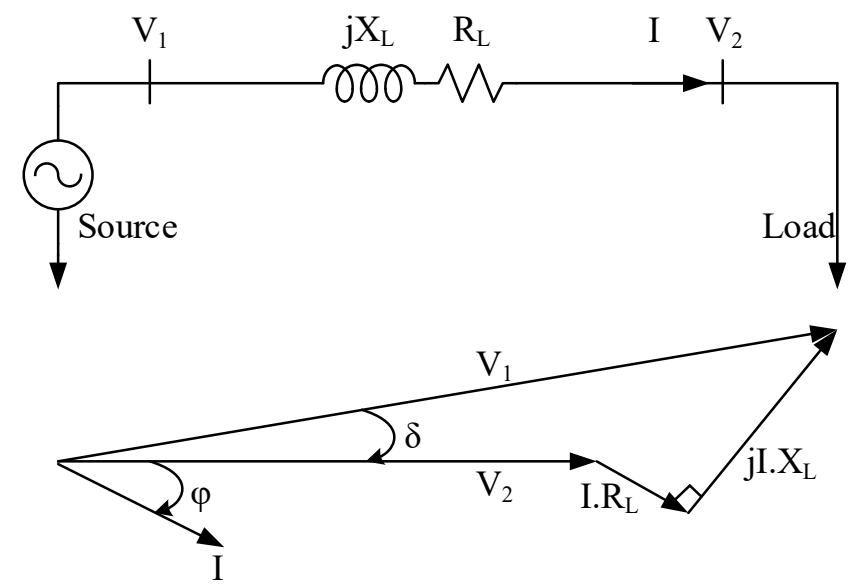

Figure 2.8: Radial AC system without reactive power compensation.

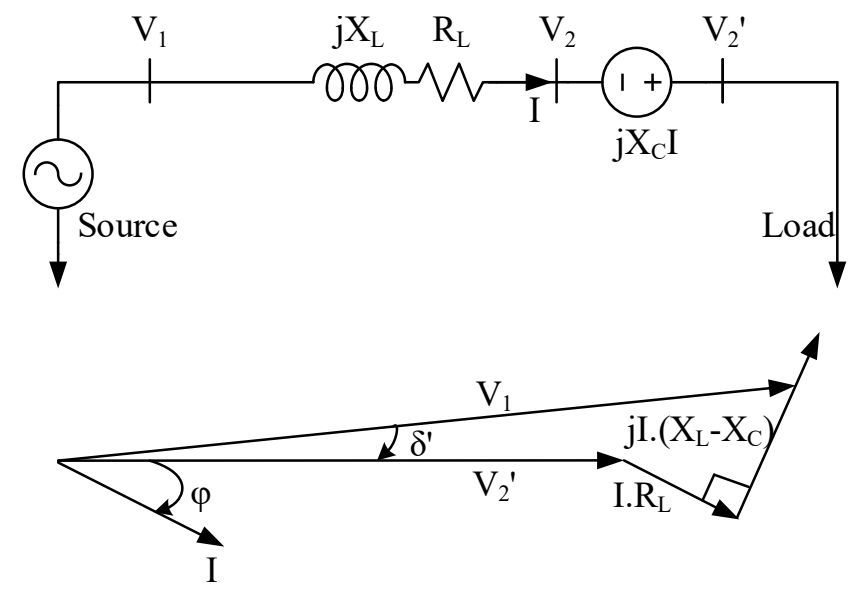

Figure 2.9: Radial AC system with series reactive power compensation.

\subsection{Static VAR Compensators (SVCs)}

Unlike shunt capacitors, shunt reactors and series capacitors, Static VAR Compensators (SVCs) provide active compensation. SVCs are shunt-connected reactive power generators or absorbers that can provide variable reactive power output. The term "static" in SVC indicates that this compensator has no moving or rotating component. Therefore, the main components of a Static VAR Compensator consist of reactive power generators (capacitors), 
reactive power absorbers (reactors), and a control system to regulate the amount of reactive power generated or absorbed by an SVC.

\subsubsection{Different Configurations of SVCs}

Based on the reactive power requirements, the following components make up all or part of a Static VAR Compensator:

- Thyristor-Controlled Reactor (TCR)

- Thyristor-Switched Reactor (TSR)

- Thyristor-Switched Capacitor (TSC)

- Fixed-Capacitor with Thyristor-Controlled Reactor (FC-TCR)

- Mechanically-Switched Capacitors or Reactors

A number of SVC configurations are possible by combining different SVC components. Figure 2.10 shows different configurations for SVC. The following sections discuss the general principles of SVC operation and then review the characteristics of commonly used configurations of an SVC.

\subsection{SVC Characteristics}

From the viewpoint of power system operation, an SVC is a device that can provide variable reactive power. It is a combination of shunt capacitors and shunt reactors, both of which are used to control the voltage and reactive power at the terminal of an SVC. An ideal SVC is capable of providing a variable amount of reactive power to support the bus voltage to which it is connected. That is, an ideal SVC possesses an unlimited 


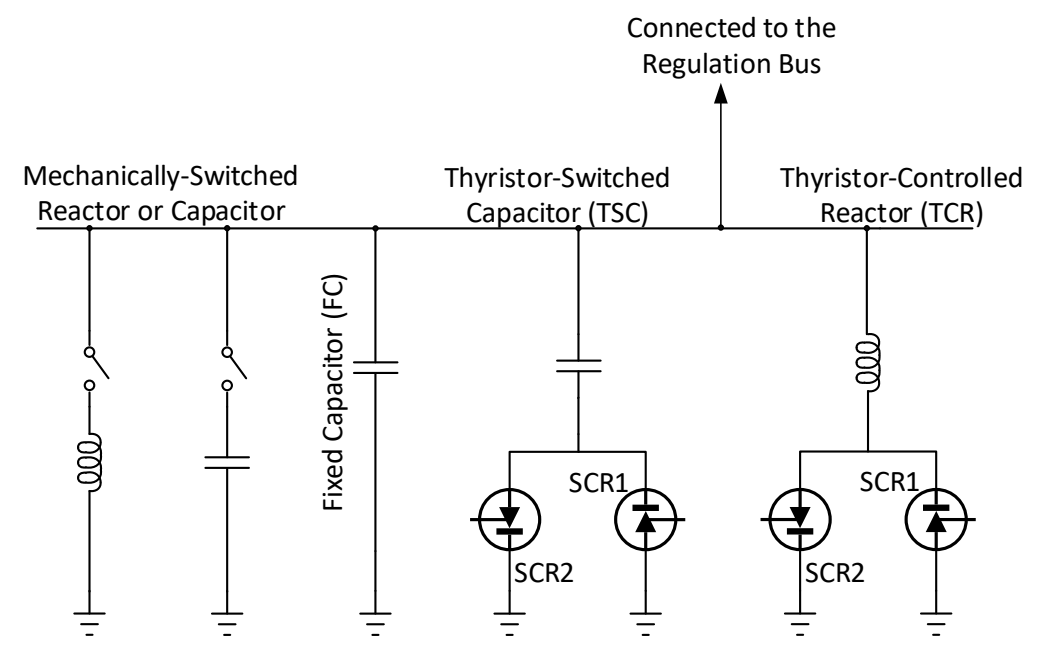

Figure 2.10: Different Configurations of Static VAR Compensator.

reactive power generation/absorption capability with no power loss during its operation. Figure 2.11a shows an ideal SVC connected to a power bus and Figure 2.11b shows the V/I characteristics of an ideal SVC which is plotted on a graph of AC bus voltage $\left(V_{B U S}\right)$ against the $S V C$ reactive current $\left(I_{S V C}\right)$. The current $I_{S V C}$ can either be capacitive or inductive depending on the requirement to regulate the bus voltage. If the $V_{B U S}$ is higher than reference voltage $V_{o}$, then an inductive current is provided by the SVC to regulate the bus voltage to $V_{o}$. Similarly, if the $V_{B U S}$ is lower than the $V_{o}$ then SVC provides capacitive current.

Practically, an SVC has a limited reactive power capability. The reactive power requirements for an SVC depend on the characteristics of the bus voltage to which it is connected. The voltage of the bus connected to an SVC is a function of the SVC current $I_{S V C}$, i.e., the voltage increases linearly with the capacitive current and decreases linearly with the inductive current. Figure 2.12 shows the V/I characteristics of a realistic SVC, which is obtained from a variable reactor with a fixed capacitor. The slope of the line $X_{S}$ represents the SVC reactance. When the bus voltage is equal to the reference voltage $V_{o}$ then the 


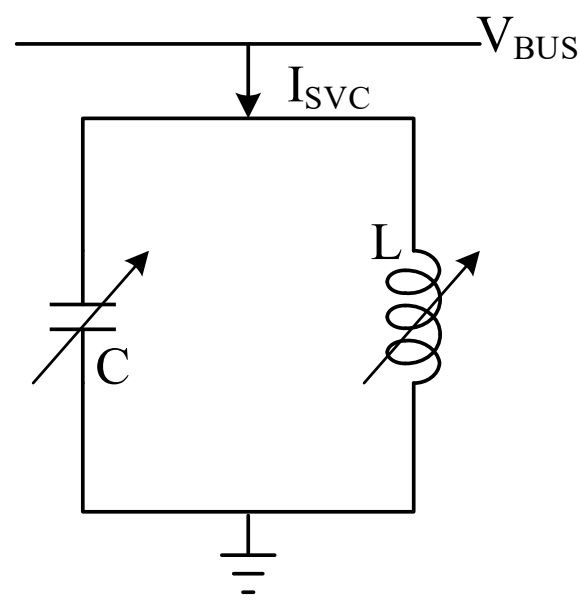

(a) Infinite variable reactive power generator

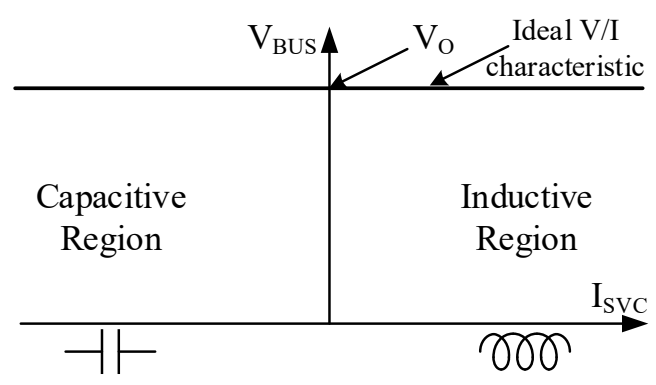

(b) V/I characteristics of an ideal Static VAR

Figure 2.11: An ideal Static VAR Compensator.

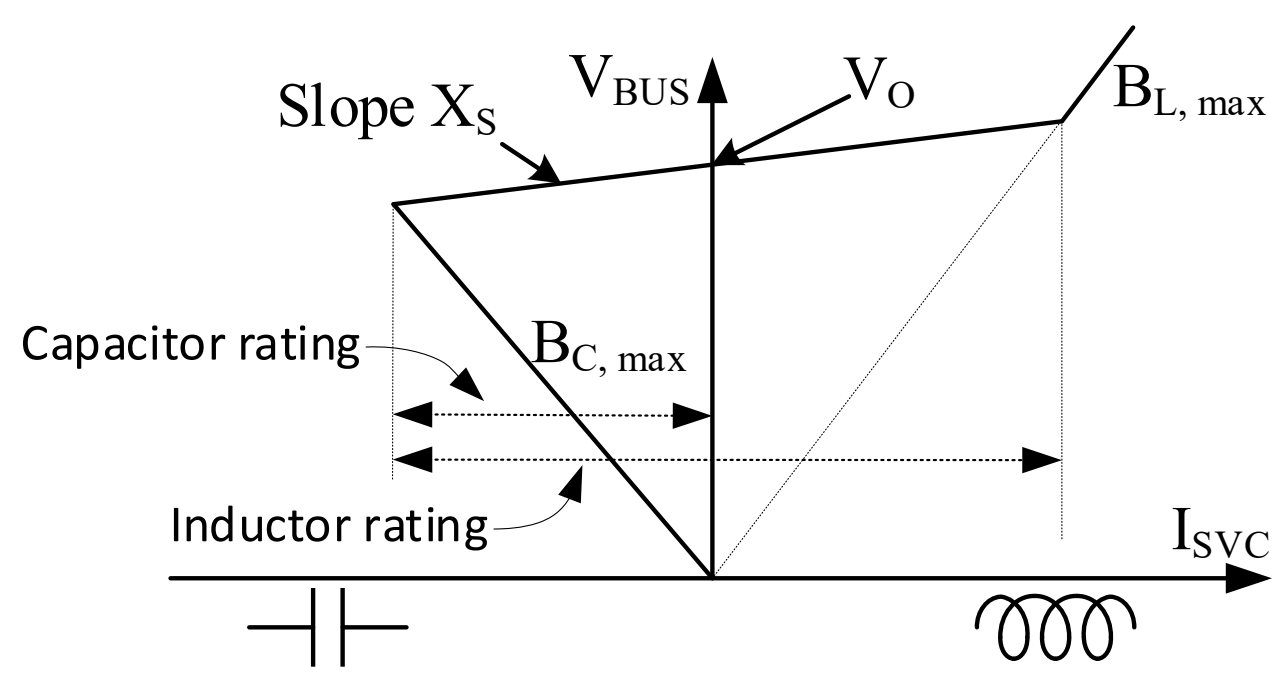

Figure 2.12: V/I characteristics of an SVC having a fixed capacitor and variable reactor.

capacitive reactance of the fixed capacitor cancels the inductive reactance of the variable reactor. When the bus voltage level is higher than $V_{o}$, an inductive current is injected by the SVC to regulate the bus voltage. However, if the bus voltage exceeds the SVC inductive limit, then $V_{B U S}$ becomes a function of inductive reactance only. Similarly, the 
SVC provides capacitive current to regulate bus voltage, when the voltage level is lower than $V_{o}$. The figure also shows the susceptance limits $B$ for the SVC. The V/I characteristics of SVC can be described by the following equations,

$$
\overline{V_{B U S}}= \begin{cases}\overline{V_{o}}+j \overline{X_{S}} \cdot \overline{I_{S V C}} & \text { when the SVC is the voltage regulation mode } \\ -j \frac{\overline{I_{S V C}}}{\overline{B_{c, \text { max }}}} & \text { when the SVC is fully capacitive } \\ j \frac{\overline{I_{S V C}}}{\overline{B_{L, \max }}} & \text { when the SVC is fully inductive }\end{cases}
$$

\subsubsection{SVC Characteristics in a Power System}

The combined characteristics of an SVC connected to the power system is shown in Figure 2.13. The middle line in the figure shows the nominal system conditions. During nominal conditions, the bus voltage is equal to $V_{o}$ and the SVC is operating at A, i.e., there is no current injected or absorbed by the SVC to maintain the bus voltage. When the system voltage increases, due to light loading conditions, the bus voltage increases from $V_{o}$ to $V_{1}$ without an SVC. With the SVC, the SVC operating point moves to point B, where it absorbs an inductive current $I_{3}$, and moves the system voltage to $V_{3}$, lower than $V_{1}$. Similarly, if the bus voltage decreases from $V_{o}$ to $V_{2}$ due to heavy loading conditions, the SVC operating point moves to point $\mathrm{C}$, where it generates a capacitive current $I_{4}$, and brings the operating point voltage to $V_{4}$ instead of $V_{2}$. As shown in the ideal SVC characteristic in Figure 2.11, if the slope of SVC characteristic is zero, the bus voltage would have been maintained at $V_{o}$ for both cases. For voltage outside the control range, the SVC operates at either of the two extremes which are determined by the ratings of the reactors and capacitors as given in Equation (2.8). 


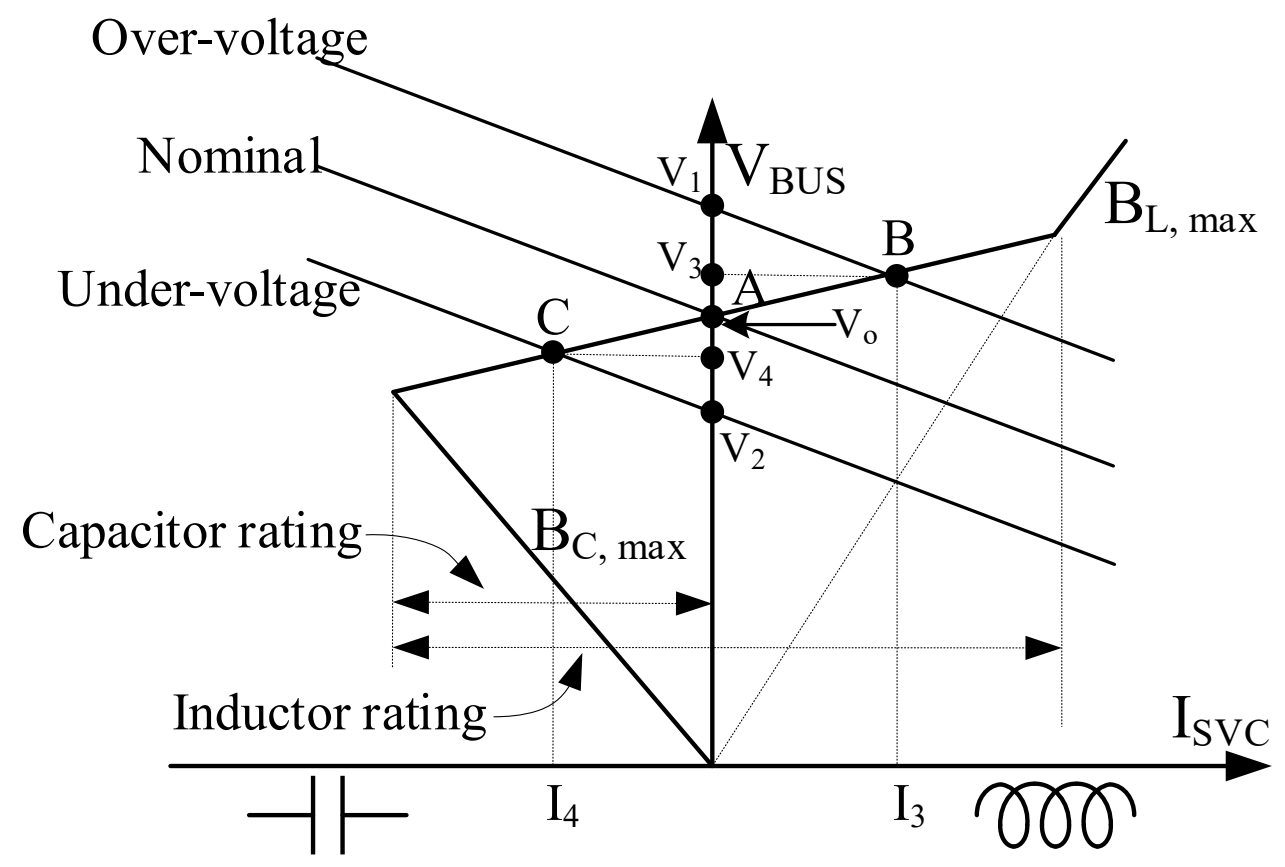

Figure 2.13: V/I characteristics of an SVC having a fixed capacitor and variable reactor with operating points for given power systems.

\subsection{Thyristor-controlled reactor (TCR)}

To understand the variable reactive power provided by the SVC, thyristor-controlled reactors (TCR) are reviewed in this section. The basic element of a TCR is a reactor which is connected in series with a bi-directional thyristor valve. Figure 2.14 shows a single-phase TCR which consists of a reactor connected in series with two thyristor valves (SCR1 and SCR2). The reactive power generated by the TCR can be varied by controlling the current flowing through the reactor. The following sections provide a detailed overview for the operational principle of a TCR and calculate the harmonics generated by TCR current. 


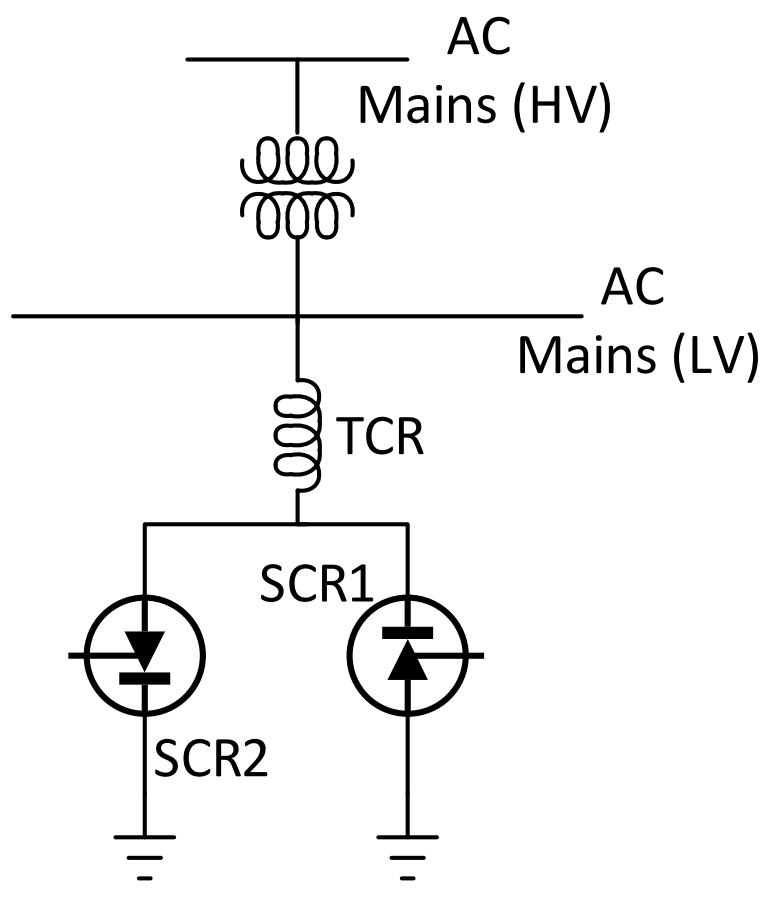

Figure 2.14: Thyristor-Controlled Reactor (TCR).

\subsubsection{Principle of operation}

Each thyristor in the valve conducts on alternate half-cycles of the supply frequency, depending on the firing angle $\alpha$, which is measured from a zero-crossing of the supply voltage. The maximum current contribution is obtained when the forward thyristor, SCR2, is fired at an angle of $\pi / 2$ and the reverse thyristor, SCR1, is fired at $3 \pi / 2$ as shown in the first plot of Figure 2.15. Partial current contribution is obtained when the firing angle $\alpha$ is varied between $\pi / 2$ and $\pi$ for SCR2 (and $\alpha+\pi$ for SCR1). The firing angles between 0 and $\pi / 2$ are not allowed, as they produce asymmetrical current with a DC component. The second plot and third plot in Figure 2.15 show the case when the firing angle is greater than $\pi / 2$. The current conduction for the TCR decreases as the firing angle approaches to $\pi$.

In order to derive the current flowing through a TCR, let $\sigma$ be the conduction angle related to the firing angle $\alpha$ by, 


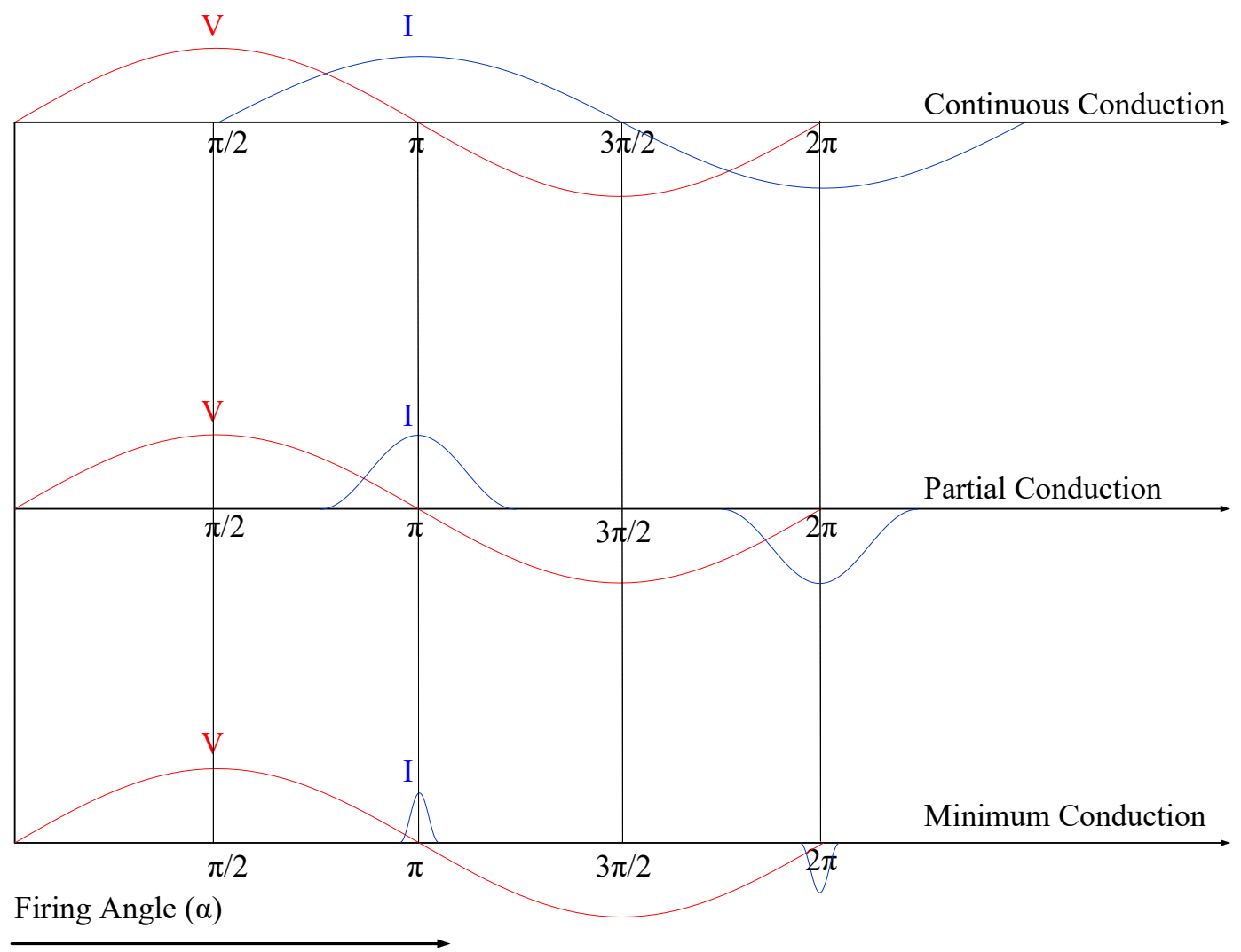

Figure 2.15: Voltage and current waveforms in a TCR for different thyristor gating angles.

$$
\sigma=2(\pi-\alpha)
$$

The instantaneous current $i(t)$ through a TCR is given by,

$$
i(t)= \begin{cases}\frac{\sqrt{2} V}{X_{L}}(\cos \alpha-\cos \omega t) & \text { for } \alpha<\omega t<\alpha+\sigma \\ 0 & \text { for } \alpha+\sigma<\omega t<\alpha+\pi\end{cases}
$$

where $\mathrm{V}$ is the RMS value of the bus voltage. Equation (2.10) can be derived from the following differential equation, 


$$
L \frac{d i}{d t}=v_{s}(t)
$$

where $L$ is the inductance of the reactor in TCR and $v_{s}(t)=\sqrt{2} V \sin \omega t$. Integrating the above equation gives,

$$
i(t)=-\frac{V}{\omega L} \cos \omega t+C
$$

where $C$ is the constant of integration which can be found by using a boundary condition when $\omega t=\alpha$. Then,

$$
i(\omega t=\alpha)=0
$$

Therefore, the instantaneous current $i(t)$ can be found as,

$$
i(t)=\frac{\sqrt{2} V}{X_{L}}(\cos \alpha-\cos \omega t)
$$

\subsubsection{Harmonic Analysis for TCR Current}

A Fourier analysis is used to calculate the fundamental component of the TCR current $i_{1}(t, \alpha)$. The Fourier series for the fundamental component of any signal is given as,

$$
i_{1}(t, \alpha)=a_{1} \cos \omega t+b_{1} \sin \omega t
$$

It can be seen that the TCR current $i(t)$ in Figure 2.15 has a quarter-wave symmetry. Therefore, the fundamental component of current waveform $i_{1}(t, \alpha)$ has even-function symmetry, i.e., $f(-t)=f(t)$ for all $t$, therefore $b_{1}=0$ and half-wave symmetry, i.e., $f(t \pm T / 2)=-f(t)$ for all $t$, therefore all even harmonics are zero. Then, the coefficient 
$a_{1}$ can be found as,

$$
a_{1}=\frac{8}{T} \int_{0}^{T / 4} f(t) \cos \omega t d t=\frac{4}{\pi} \int_{0}^{\pi / 2} f(\theta) \cos \theta d \theta
$$

Solving the integral in Equation (2.16) from firing angle $\alpha$ to $\pi$ by using Equation (2.14) gives the following expression of the fundamental component of the thyristor current,

$$
I_{1}(\alpha)=\frac{V}{\omega L}\left(\frac{2 \pi-2 \alpha+\sin 2 \alpha}{\pi}\right)
$$

In terms of the conduction angle $\sigma$, the fundamental component of the TCR current is given as,

$$
I_{1}(\sigma)=\frac{V}{\omega L}\left(\frac{\sigma-\sin \sigma}{\pi}\right)
$$

The current can also be written in terms of susceptance $B$ as,

$$
I_{1}(\sigma)=V B_{T C R}(\sigma)
$$

where

$$
B_{T C R}(\sigma)=B_{\max }\left(\frac{\sigma-\sin \sigma}{\pi}\right)
$$

and

$$
B_{\max }=\frac{1}{\omega L}
$$


Figure 2.16 shows the variation of the per unit value of $B_{T C R}$ with the firing angle $\alpha$. As susceptance is the measure of how much a circuit is susceptible to conducting current, the plot in Figure 2.16 shows that the ability to conduct current by the TCR decreases as the firing angle $\alpha$ increases. In other words, a thyristor-controlled reactor acts as variable susceptance (or variable reactance) device. The variation of susceptance changes the fundamental current component which leads to a variable reactor power absorbed by the reactor.

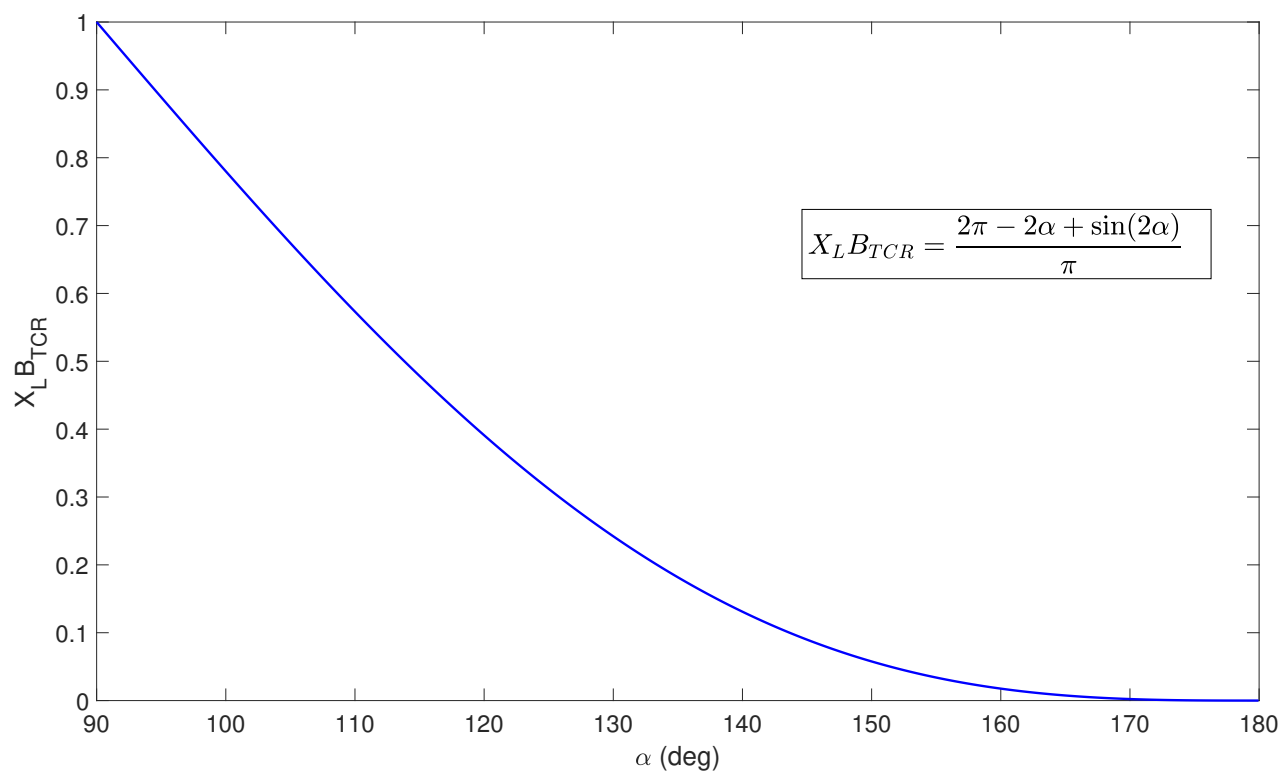

Figure 2.16: Variation of the TCR susceptance $B_{T C R}$ with firing angle $\alpha$.

For nth-order harmonics, if the anti-parallel thyristors in the TCR are fired at the same firing angles in the positive and negative half-cycles of the source voltage, then even harmonics are not generated due to an even quarter-wave symmetry (i.e., a combination of an even-function symmetry plus a half-wave symmetry). Consequently, only the nth-order odd harmonics appear in the TCR current. These harmonics can be computed using a 
Fourier series as follows,

$$
I_{n}=\frac{4}{\pi} \int_{0}^{\pi / 2} f(\theta) \sin \theta d \theta=-\frac{V}{\omega L} \frac{4}{\pi} \int_{\alpha}^{\pi}(\cos \alpha-\cos \theta) \cos n \theta d \theta
$$

The above equation can be separated into two integrals such as,

$$
I_{n}=-\frac{V}{\omega L} \frac{4}{\pi} \int_{\alpha}^{\pi}(\cos \alpha \cos n \theta d \theta-\cos \theta \cos n \theta d \theta)
$$

Using trigonometric identities, the nth-order harmonics are expressed as,

$$
\begin{aligned}
I_{n}(\alpha) & =-\frac{V}{\omega L} \frac{4}{\pi}\left[\frac{\cos \alpha \sin n \alpha}{n}-\frac{\sin (n-1) \alpha}{2(n-1)}-\frac{\sin (n+1) \alpha}{2(n+1)}\right] \\
& =\frac{V}{\omega L} \frac{4}{\pi}\left[\frac{\sin \alpha \cos (n \alpha)-n \cos \alpha \sin (n \alpha)}{n\left(n^{2}-1\right)}\right]
\end{aligned}
$$

where $n=3,5,7, \ldots$ Figure 2.17 shows the variation of the amplitude of different harmonics in the TCR current. It can be seen that the harmonics do not peak at the same firing angle. The peak values for different harmonic currents are also expressed as the percentage of the fundamental component in Figure 2.17. Figure 2.18 shows the THD in the TCR current at different firing angles. The plot also shows the decay of the fundamental component of the TCR current with the firing angle.

Thyristor-controlled reactors have the desirable property of variable reactance but due to the presence of lower-order harmonics, single-phase TCR are generally not employed in distribution and residential feeders. 


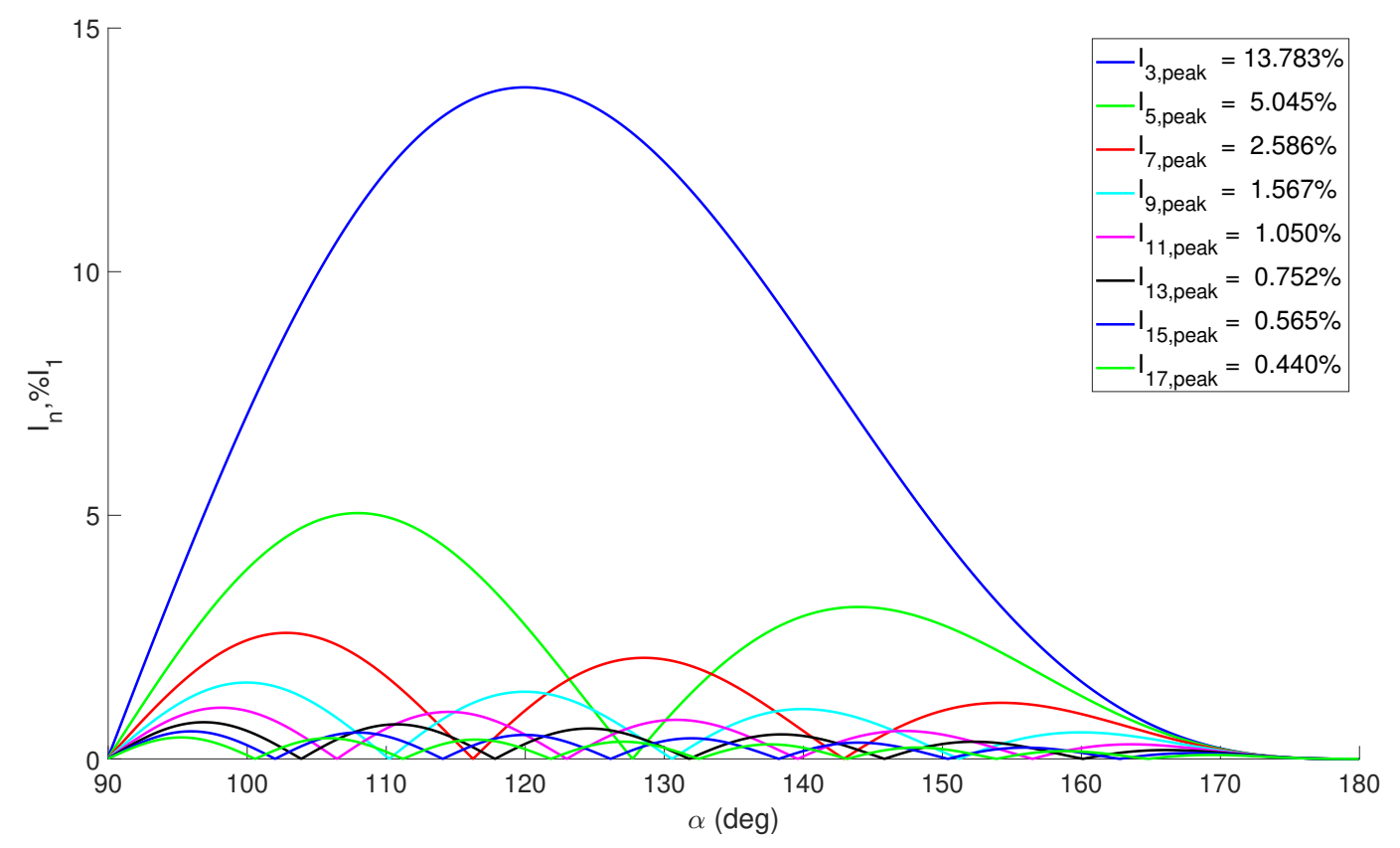

Figure 2.17: Harmonics in the TCR current.

\subsubsection{Three-Phase Thyristor-Controlled Reactor}

A three-phase TCR consists of three single-phase TCRs connected in a delta configuration. Each leg of a three-phase TCR consists of a reactor connected with two anti-parallel thyristors, therefore requiring a total of six gate firing pulses. If the phases in a three-phase TCR consists of identical reactors and are fed from a balanced three-phase voltage supply, then the harmonic currents generate only odd harmonics given that the thyristors are fired symmetrically.

The primary purpose of using the delta configuration of the three single-phase TCR is to prevent triplen-harmonics (harmonics which are multiples of three) to get into the power system. The elimination of the third and its multiple harmonics from the power system can be explained using Figure 2.19. Let $i_{A B}, i_{B C}$ and $i_{C A}$ represent the phase currents in the delta 


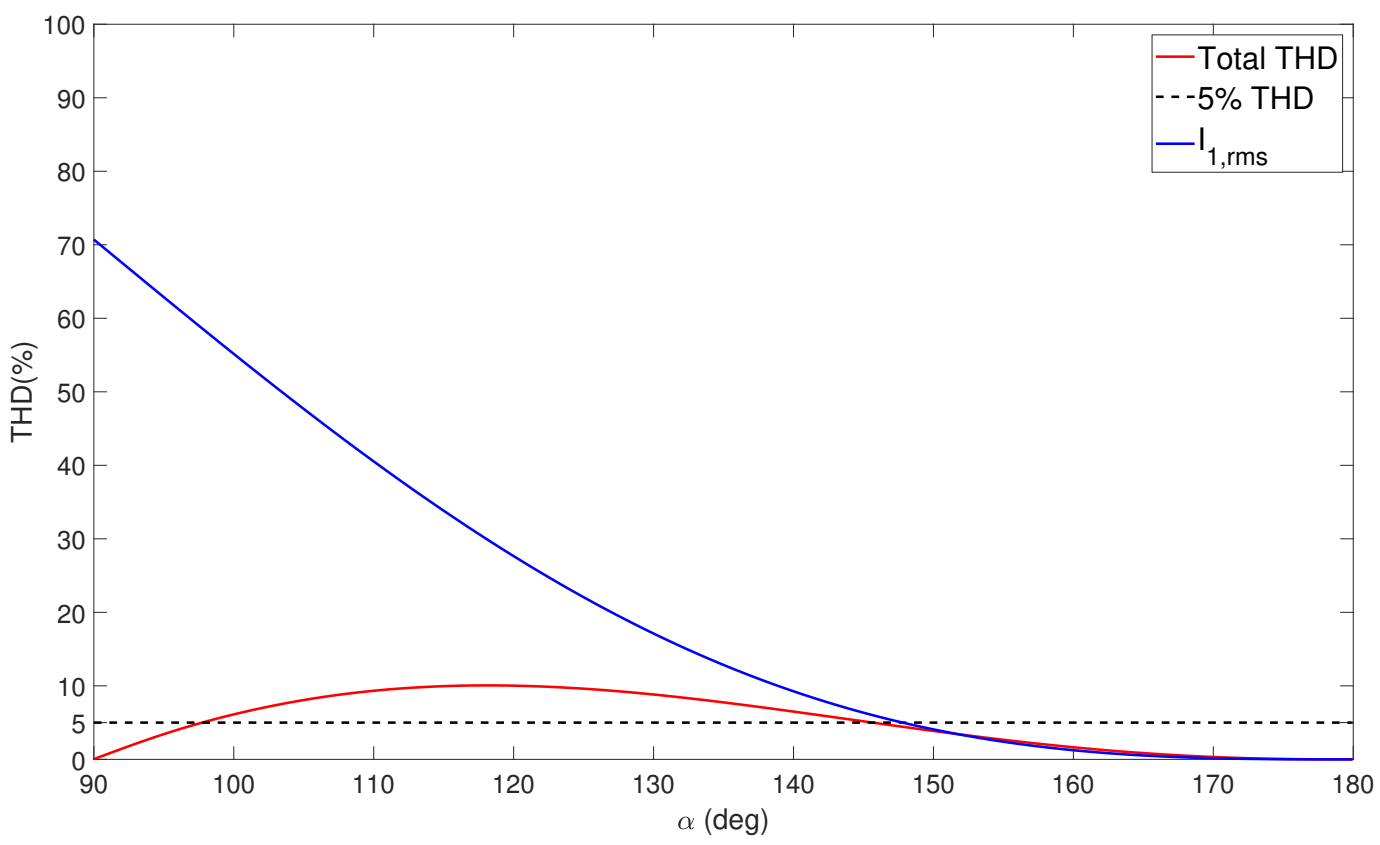

Figure 2.18: Total Harmonic Distortion (THD) for the TCR current.

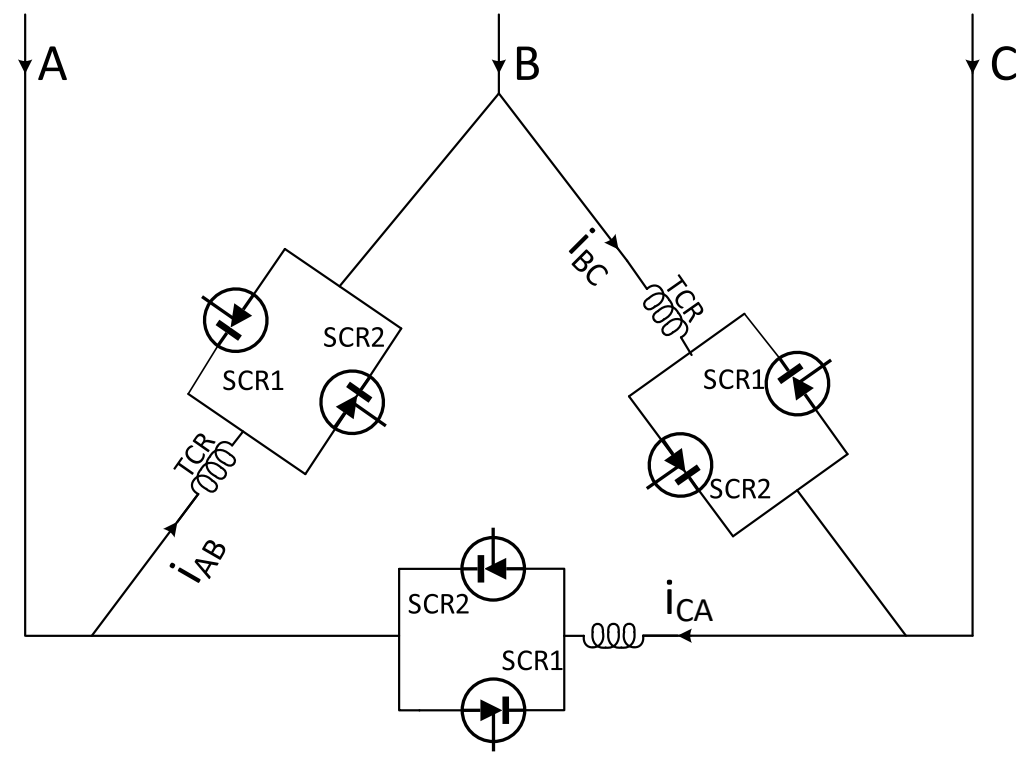

Figure 2.19: A delta-connected three-phase TCR. 
connection and $I_{A}, I_{B}$ and $I_{C}$ represent the line currents in the respective lines connected to the 3-phase delta connection. The nth-order harmonic currents are expressed as,

$$
\begin{aligned}
& i_{A B, n}=a_{n} \cos \left(n \omega t+\phi_{n}\right) \\
& i_{B C, n}=a_{n} \cos \left(n \omega t+\phi_{n}-n \frac{2 \pi}{3}\right) \\
& i_{C A, n}=a_{n} \cos \left(n \omega t+\phi_{n}+n \frac{2 \pi}{3}\right)
\end{aligned}
$$

For triplen-harmonics currents, $n=3 k+3$ and $k=0,2, \ldots$ The nth-order harmonic line current $i_{A, n}$ can be calculated as follows,

$$
i_{A, n}=i_{A B, n}-i_{C A, n}=0
$$

Similarly, $i_{B, n}$ and $i_{C, n}$ can also be calculated. For triplen-harmonic components of the TCR current, the line currents $i_{A,(3 k+3)}, i_{B,(3 k+3)}$ and $i_{C,(3 k+3)}$ reduce to zero. Therefore, the third and multiple of third harmonics generated as a result of the non-sinusoidal TCR current is trapped in the delta configuration and cannot flow into the lines due to balanced operation.

In general, three-phase TCRs are provided with LC or LRC filters in shunt. These filters are tuned to cancel the dominant $5^{\text {th }}$ and $7^{\text {th }}$ order harmonic frequencies. For a single-phase TCR, a $3^{r d}$-order harmonic filter needs to be installed in shunt with the TCR to eliminate the dominant harmonic components. Moreover, the change in reactor current can only happen once during each half cycle. This lack of controllability of the reactive power during a complete cycle is another drawback for the thyristor-controlled reactor. The thyristor gating signals must stay synchronized with the AC mains voltage under all circumstances. This synchronization is achieved by using a phase-locked loop (PLL) that operates synchronously with the AC system voltage and produces a thyristor gate-firing 
sequence with respect to the peak of that voltage. The control system responsible for the PLL must be fast enough to respond during system faults and voltage fluctuations. The use of a synchronization block for producing gate signals not only poses technical difficulties but also increases the total components required for building a single-phase SVC.

\subsection{Thyristor-Switched Reactor (TSR)}

The thyristor-switched reactor (TSR) is a special case for the TCR. If the thyristors valves in the TCR are fired at the instants when the voltage peak occurs in the positive and negative half-cycles, it results in the full conduction of TCR current, as shown in Figure 2.15. Therefore, for $\alpha=\pi / 2$ for positive and $\alpha=\pi$ in negative half-cycles; maximum current flows in the TCR. However, if no firing pulses are applied, then no current flows through the TCR. The thyristor-switched reactor operates in the same manner as a TCR. However, instead of using two anti-parallel thyristor switches, the TSR uses a single thyristor switch. A major drawback of using the TSR is that instead of getting a variable reactive power as in the case of the TCR, the TSR operates in two states only, i.e., it is either fully on or fully off.

When a large magnitude of inductive reactive power, $Q_{L}$, is required, a part of it is supplied by the TSR, $Q_{L, T S R}$, and the rest is supplied by the TCR, $Q_{L, T C R(\alpha)}$. The total inductive reactive power for an SVC arrangement involving a TSR and TCR is given as,

$$
Q_{L}=Q_{L, T S R}+Q_{L, T C R}(\alpha)
$$

This arrangement results in reduced harmonic content compared to a single TCR of a similar reactive power rating. However, the harmonics reduction is achieved by an increased cost of thyristor switches. 


\subsection{Thyristor-Switched Capacitor (TSC)}

A thyristor-switched capacitor (TSC) consists of a capacitor bank, which is switched using a thyristor valve. A typical TSC consists of a capacitor $C$ in series with a bi-directional thyristor valve and a small inductor $L$. The inductor $L$ is used to limit the switching transients and to prevent resonance with the power network.

\subsubsection{Principle of Operation}

In order to avoid capacitor switching transients, the thyristors are switched when the voltage across them is at a maximum. Therefore, the thyristor firing control system is designed such that the thyristors are fired at the positive or negative peak of the supply voltage. Figure 2.20 shows the operating principle of the switching operation of a TSC. The thyristors are fired at time instant $t_{1}$, which is chosen such that the bus voltage $V$ is equal to the capacitor voltage $V_{c}$ in terms of magnitude and polarity. When it is required to open the capacitor bank, the capacitor banks are switched out at the zero-crossing of the capacitor current $I_{c}$, i.e., when the $V=V_{c}$.

\subsubsection{Dynamic Response with Multiple TSCs}

A thyristor-switched capacitor, in its simplest configuration, consists of a single capacitor bank. The size of the capacitor bank can be calculated based on the reactive power requirements $Q_{C}$ as,

$$
C_{r e q}=\frac{V^{2}}{\omega Q_{C}}
$$




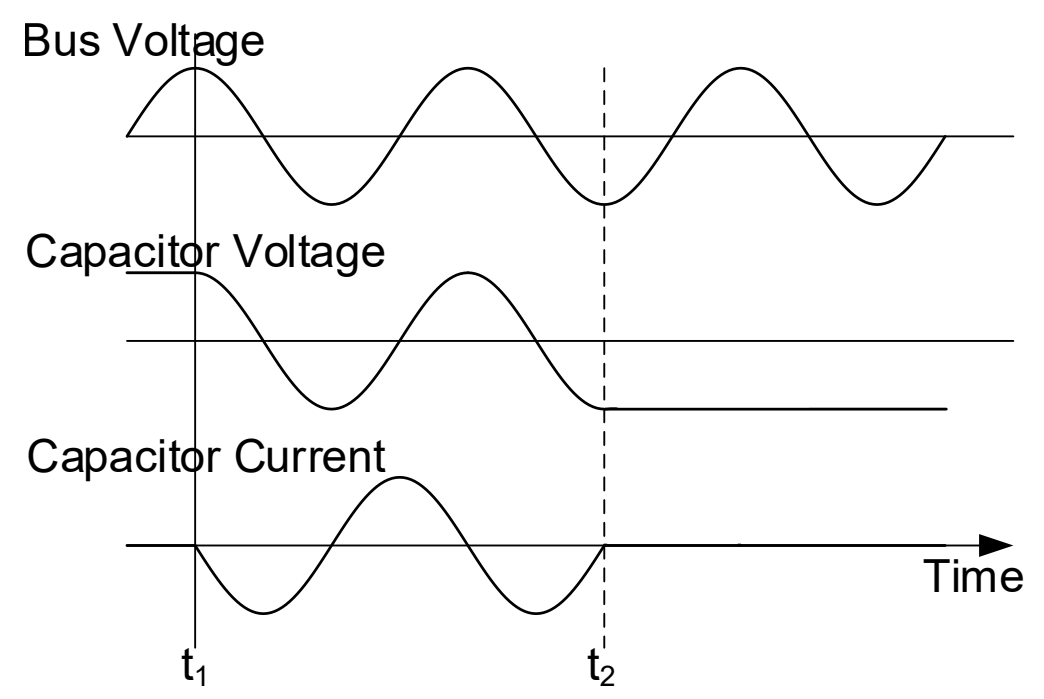

Figure 2.20: Switching operation of a TSC.

where $\omega$ is the frequency of the supply voltage. However, instead of using a single capacitor bank, multiple TSC branches can be used. An SVC can have $n$ different values for capacitors on different TSC branches. In this way, there are $2^{n}$ discrete compensation levels, including the zero reactive power level, when all TSC branches are disconnected.

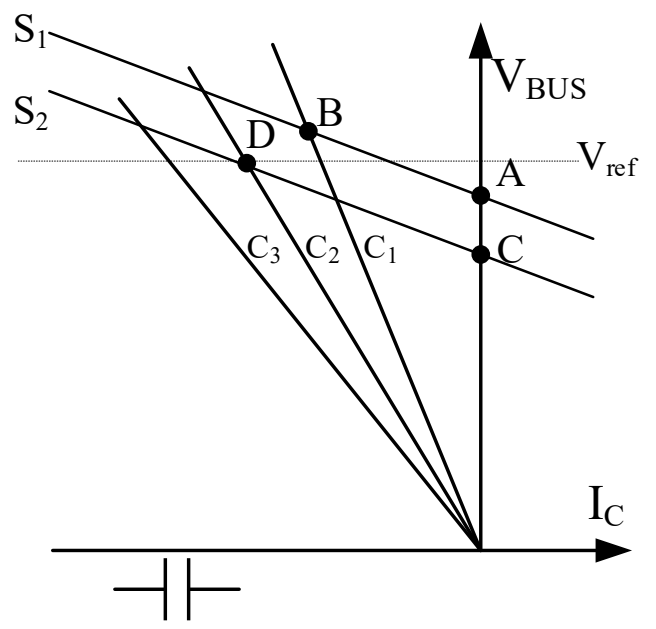

Figure 2.21: V/I characteristics of a multiple TSC with operating points for given power systems. 
Figure 2.21 shows the V/I characteristics of an SVC having three TSC branches. The voltage regulation provided by such an SVC is discontinuous or step-wise. In the figure, $S_{1}$ and $S_{2}$ represent the power system characteristics. At a certain instant of time, the power system is operating so that its characteristic is represented by line $S_{1}$ and the voltage of the bus is at point $A$. At this operating point, capacitor $C_{1}$ is turned on to provide reactive power support and the bus voltage increases to point B. After a certain time, the system characteristics changes from $S_{1}$ to $S_{2}$ and the power system is now operating at point C. In this case, the capacitor bank $C_{2}$ will turn on and change the operating point to $D$, as shown in the figure.

Despite the simple construction and working principle of the TSC, it is not widely adopted due to the following disadvantages

- the reactive power compensation provided is not continuous and compensation can only happen in discrete steps,

- each TSC branch in an SVC requires a separate thyristor valve and therefore it is not economical,

- the voltage across the non-conducting thyristor in the thyristor valve is twice as high as the peak supply voltage and thus requires high power rated thyristors.

Due to these issues, the fixed-capacitor with thyristor-controlled reactor (FC-TCR) becomes an economically viable option for providing reactive power compensation in the power system. 


\subsection{An Efficient Switched-Reactor-Based Static VAR Compensator}

The Static VAR Compensators (SVCs) currently used in electric utilities are mainly based on thyristor-controlled reactors (TCRs) with fixed or thyristor-switched capacitors. In order to achieve both leading and lagging reactive power regulation, a single fixed capacitor (FC) or multiple thyristor-switched capacitors are used together with a controlled reactor. TCR-based compensators have simple power structures and control schemes. However, they generate low-order harmonics and often require additional filters. Additionally, the response time of the compensator is limited by the delay in thyristor gating control.

This section introduces a novel pulse-width modulation (PWM) based SVC which includes a PWM-based switched reactor with a fixed capacitor to provide the voltage regulation and reactive power compensation at the point of common coupling. The PWM-based switched reactor is provided with a free wheeling current path which conducts the reactor current when the reactor is disconnected from the power circuit. The PWM-based SVC is capable of providing reactive power while keeping low harmonic injections in a power system. Moreover, the PWM-based SVC can operate at higher switching frequencies with a significantly faster response time compared to a conventional SVC based on thyristor gate firing.

\subsubsection{PWM-Based Switched Reactor}

Figure 2.22 shows a single-phase, PWM-based switched reactor topology. A detailed analysis for this topology can also be found in the literature [36]. The single-phase circuit requires two bi-directional switches, each of which is built using two unidirectional switches and two diodes, as explained in Chapter 3. In this figure, the switches SW1 and SW2 represent two bi-directional switches with complementary gating signals. The switch 


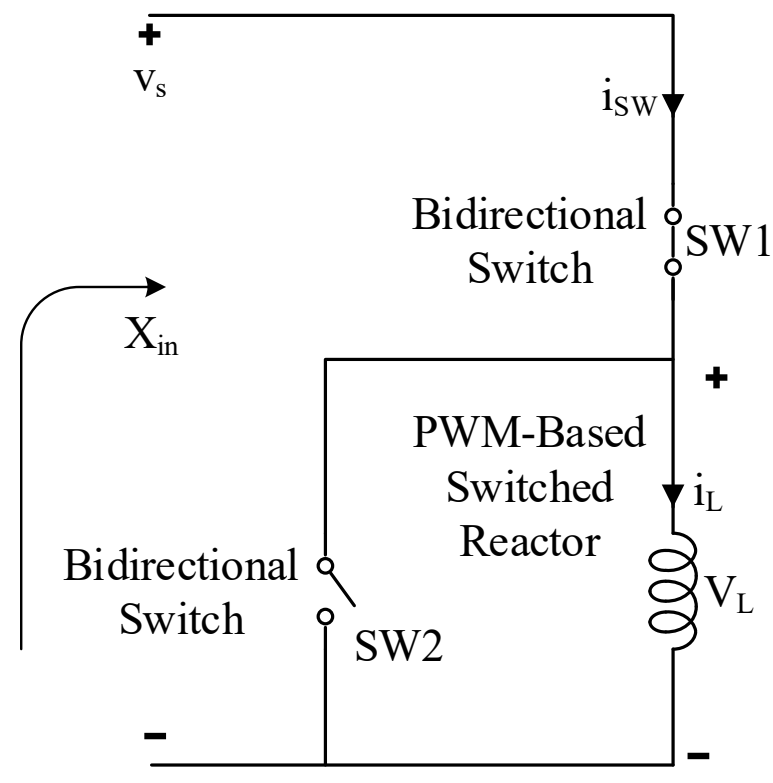

Figure 2.22: PWM-based switched reactor

SW2 is used to conduct the inductor current when switch SW1 is open and vice versa. By using high frequency switching, the fundamental component of the inductor current can be controlled. Assuming the switches to be ideal, meaning their switching and conduction losses are negligible, when SW1 is closed (SW2 is open), a voltage $V_{L}$ appears across the inductor. It can be shown that the RMS magnitude of the fundamental component of this voltage is related to the RMS magnitude of the quasi-sinusoidal source voltage $V_{S, R M S}$ by the following expression,

$$
V_{L 1, R M S}=D V_{S, R M S}
$$

where $D$ is the duty cycle ratio defined as time interval when SW1 is conducting. Similarly, the RMS magnitude of the fundamental component of the top switch current $I_{S W 1, R M S}$ is related to the RMS magnitude of the quasi-sinusoidal inductor current by the following expression,

$$
I_{S W 1, R M S}=D I_{L 1, R M S}
$$


From the above equations, the equivalent input inductive reactance $X_{\text {in }}$ can be found by using the following expression,

$$
X_{i n}(D)=\frac{V_{S, R M S}}{I_{S W 1, R M S}}=\frac{V_{L 1, R M S} / D}{D I_{L 1, R M S}}=\frac{X_{L}}{D^{2}}
$$

From Equation (2.31), it can be seen that $X_{i n}(D)$ can be controlled by the duty cycle $D$. This makes the fixed inductor reactance to appear like a variable reactance as a function of duty cycle. The dynamic reactive power for the PWM-based switched inductor can be expressed by the following expression,

$$
Q_{L}(D)=\frac{V_{s}^{2}}{X_{i n}(D)}
$$

By increasing the duty cycle, the input reactance $X_{\text {in }}$ decreases, while the inductive reactive power increases. There is a noticeable improvement in the performance of the PWMbased switched inductor compared to the conventional TCR due to the presence of an alternating path for the inductor current through switch SW2 which is helpful in keeping the inductor current quasi-sinusoidal. This improvement eliminates the requirement for additional filtering circuits that are required for limiting the harmonics in the conventional TCR. The reactive power injected by the PWM-based switched inductor is continuously variable. It can be varied at any time during each cycle by varying the duty cycle $D$ of switch SW1. This fact also eliminates the need for a synchronization block that is required for providing gating signals to thyristors. Due to lower power ratings for the reactive components involved and reduction in the total components required (the harmonic filtering) to realize this device, PWM-based SVC is economically more feasible than a conventional SVC. Using this new approach, utilities can benefit with having the same 
characteristics of a TCR-FC without polluting the AC system with low-order harmonics.

\subsubsection{Switching the Current Harmonic Distortion for the PWM-Based Switched Reactor}

Let $v_{S}(t)$ be the input source voltage and $i_{L}(t)$ be the current flowing through the inductor as shown in Figure 2.22. Assume that the source voltage $v_{s}(t)$ is quasi-sinusoidal and of the form,

$$
v_{s}(t)=\sqrt{2} V_{s 1} \sin (\omega t)
$$

where $V_{s 1}$ is the RMS value of the fundamental component of the source voltage. Similarly, assume a quasi-sinusoidal inductor current $i_{L}(t)$,

$$
i_{L}(t)=\sqrt{2} I_{L 1} \sin \left(\omega t-90^{\circ}\right)
$$

where $I_{L 1}$ is the RMS value of the fundamental component of the inductor current. The current through the switch SW1 is the product of the inductor current $i_{L}(t)$ and the switching function given by the gating signal for the SW1. The switching function is a PMW function $f(t)$ whose Fourier series analysis is provided in Appendix A. The switching function $f(t)$ can be expressed as,

$$
f(t)=a_{0}+\sum_{n=1}^{\infty} a_{n} \cos \left(n k_{f} \omega t\right)=D+\sum_{n=1}^{\infty} \frac{2}{n \pi} \sin (n \pi D) \cos \left(n k_{f} \omega t\right)
$$

where D is the duty cycle of the switch SW1 and $k_{f}$ is the ratio between the switching frequency $f_{s w}$ and the fundamental frequency of $60 \mathrm{~Hz}$, i.e., $k_{f}=f_{s w} / 60$. Using Equation (2.33) and Equation (2.34), the switching current can be computed as follows, 


$$
\begin{aligned}
i_{s w}(t) & =f(t) i_{L}(t) \\
& =\left(D+\sum_{n=1}^{\infty} \frac{2}{n \pi} \sin (n \pi D) \cos \left(n k_{f} \omega t\right)\right) i_{L}(t)
\end{aligned}
$$

Once the switching harmonics greater than $f_{s w}$ are neglected then $i_{s w}(t)$ is given as,

$$
i_{s w}(t) \cong\left(D+\frac{2}{\pi} \sin (\pi D) \cos \left(k_{f} \omega t\right)\right)\left(\sqrt{2} I_{L 1} \sin \left(\omega t-90^{\circ}\right)\right)
$$

Assuming the inductor current is sinusoidal, the sum of the higher harmonics in the inductor current can be neglected. Therefore, the current through the switch $i_{s w}(t)$ can be simplified as follows,

$$
i_{s w}(t)=\sqrt{2} D I_{L 1} \sin \left(\omega t-90^{\circ}\right)-D_{1} I_{L 1}\left(\cos \left(\omega t\left(k_{f}-1\right)\right)+\cos \left(\omega t\left(k_{f}+1\right)\right)\right)
$$

where $D_{1}=4 \sin (\pi D) / \pi \sqrt{2}$. The fundamental component of the current through switch SW1 is given by the first term of the switching current $i_{s w}(t)$ at the frequency of the source frequency $\omega t$. Therefore, the RMS value of the fundamental component of the input current is given as,

$$
I_{S W 1, R M S}=D I_{L 1, R M S}
$$

This result is similar to the result mentioned in Equation (2.30). The result in Equation (2.37) is important as it indicates that the harmonics generated by switching an inductor depends on the switching frequency $f_{s w}$. The current harmonics produced by the PWMbased reactor switching are moved to the switching frequency band as opposed to the lower order current harmonics generated by the thyristor current reactor due to the non-sinusoidal inductor current. 


\subsubsection{Input Current Harmonic Distortion for PWM-Based Switched Reactor}

The fundamental component of the input current $i_{i n, 1}$ is given by the first term of the switching current in Equation (2.37) and the fundamental component of the capacitor current as,

$$
\begin{aligned}
i_{i n, 1} & =i_{s w, 1}+i_{c, 1} \\
& =\sqrt{2} D I_{L 1} \sin \left(\omega t-90^{\circ}\right)+\frac{V_{s}}{X_{c}} \sin \left(\omega t+90^{\circ}\right) \\
& =V_{s}\left(\frac{D^{2}}{X_{L}}-\frac{1}{X_{c}}\right) \sin \left(\omega t-90^{\circ}\right)
\end{aligned}
$$

The harmonic component of the input current $i_{i n, n}$ is calculated with the help of Figure 2.23. In this figure, $i_{i n, n}$ shows the harmonics of the input current, $\left(n_{k f} \pm 1\right) X_{T}$ shows the equivalent leakage reactance, $X_{c} /\left(n_{k f} \pm 1\right)$ shows the equivalent fixed capacitor reactance and $i_{s w, n}$ represents the harmonics of the switching current as given by Equation (2.37). Note that Equation (2.37) only shows the fundamental component of the switch current and the components of the switching current present at the switching frequency. The harmonics of the input current $i_{i n, n}$ can be calculated as follows,

$$
\begin{aligned}
i_{i n, n} & =\left[\frac{X_{c} /\left(n k_{f}+1\right)}{X_{T}\left(n k_{f}+1\right)+X_{c} /\left(n k_{f}+1\right)}+\frac{X_{c} /\left(n k_{f}-1\right)}{X_{T}\left(n k_{f}-1\right)+X_{c} /\left(n k_{f}-1\right)}\right] i_{s w, n} \\
& =\left[\frac{1}{1+\frac{X_{T}}{X_{c}}\left(n k_{f} \pm 1\right)^{2}}\right] i_{s w, n}
\end{aligned}
$$

If $n=1$ in Equation (2.40), then $i_{i n, 1}$ is given as,

$$
i_{i n, 1}=\left[\frac{1}{1+\frac{X_{T}}{X_{c}}\left(k_{f} \pm 1\right)^{2}}\right] i_{s w, 1}
$$




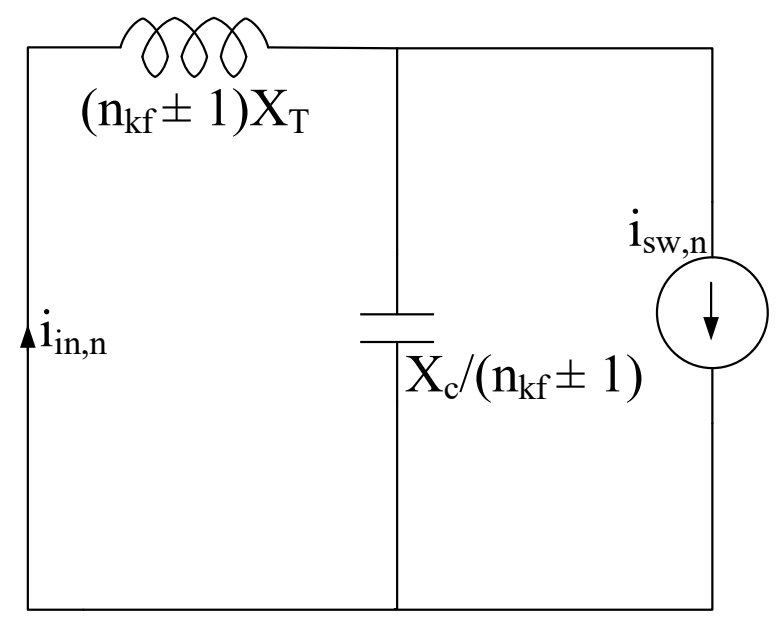

Figure 2.23: The circuit for calculating the input current $i_{i n, n}$ harmonics.

From Equation (2.41), it can be seen that the input harmonic current only exists at the switching frequency band. Hence, the principle advantage of a PWM-based Static VAR Compensator lies in its ability to move the harmonics, created by a single-phase PWMbased SVC, into the switching frequency band as compared to the lower-order harmonics that existed in a single-phase conventional thyristor-based SVC, as shown in Equation (2.24).

\subsubsection{The Reactive Power Capability of a PWM-Based Switched Reactor with a Fixed Capacitor}

Figure 2.24 shows the structure for a single-phase compensator with a PWM-based switched reactor and fixed capacitor. The switches are turned on in a complementary fashion to provide a free-wheeling path for the reactor current. The net reactive power supplied or generated by the compensator $Q_{S V C}$ can be calculated as,

$$
Q_{S V C}=Q_{C}-Q_{L}(D)=V_{s}^{2}\left(\frac{1}{X_{C}}-\frac{D^{2}}{X_{L}}\right)
$$




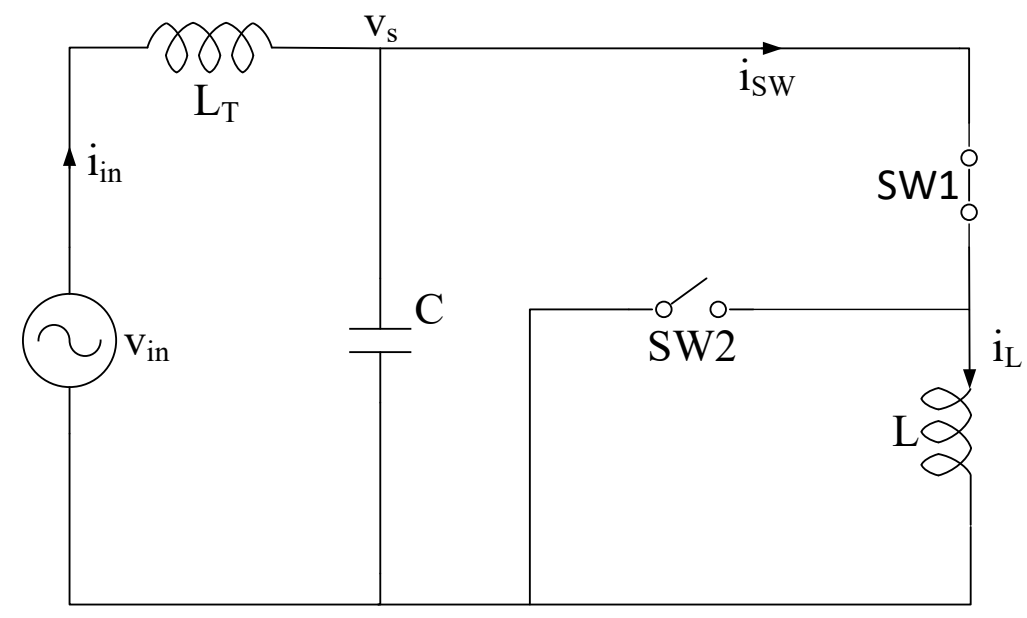

Figure 2.24: Single-phase PWM-based switched reactor with fixed capacitor.

where $Q_{C}$ is the reactive power supplied by the capacitor, $Q_{L}(D)$ is the reactive power absorbed by the reactor as a function of duty cycle, $D$ is the duty cycle of the $\mathrm{SW} 1, V_{S}$ is the fundamental component of the capacitor voltage, $X_{C}$ is the capacitor reactance given as $X_{C}=1 / \omega C$, and $X_{L}$ is the nominal inductor reactance given as $X_{L}=\omega L$. It is to be noted that the capacitors not only provide the leading reactive power but also aid in reducing the high-frequency harmonics. This scheme helps to eliminate the use of additional filters and thus simplify the overall single-phase SVC structure.

\subsubsection{Design Example}

In order to present the design process, a per-unit (pu) system is used. The voltage rating for the secondary side of the transformer and its power ratings are used as the base values. The design procedure is presented for a compensator that can provide a reactive power change from $1 \mathrm{pu}$ (lagging) to $1 \mathrm{pu}$ (leading). The design task is to find the capacitance $C$, the reactor $L$ and the switching frequency $f_{s w}$ that satisfies the above mentioned requirements. As capacitors are used to provide the leading reactive power, the inductor is completely 
disconnected, i.e., the duty cycle for SW1 is zero. Therefore, the reactive power of the capacitance can be calculated as,

$$
Q_{S V C}=Q_{C}=\frac{V_{s}^{2}}{X_{C}}=\omega C V_{s}^{2}
$$

Since, $V_{s}=1 \mathrm{pu}$ and $Q_{c}=1 \mathrm{pu}$, therefore $X_{c}=1 \mathrm{pu}$. For the lagging reactive power of 1 p.u., the switch SW1 remains on all the time. Therefore, the reactive power is given as follows,

$$
\begin{aligned}
Q_{S V C} & =Q_{C}-\left.Q_{L}(D)\right|_{D=1} \\
& =V_{S}^{2}\left(\frac{1}{X_{C}}-\frac{1}{X_{L}}\right)
\end{aligned}
$$

Using $V_{s}=1$ p.u., $Q_{c}=-1$ p.u. and $X_{c}=1$ p.u., the inductive reactance $X_{L}$ is equal to 0.5 p.u. The leakage inductance of the transformer $L_{T}$ also has a significant effect on the performance of the transformer. A typical distribution transformer has a leakage inductance value of $5 \%$ p.u. For the purpose of this analysis, the role of $L_{T}$ is ignored. The overall reactive power given by the proposed PWM-based SVC can be plotted as a function of the duty cycle as shown in Figure 2.25. The figure shows that for the duty cycle less than 0.71 , the compensator behaves in the capacitive region. Above 0.71, the compensator provides inductive reactive power. At duty cycle $D=0.71$, the leading and lagging currents cancel each other.

To conclude, this chapter proposes an improved device for shunt compensation. The proposed compensator consists of a fixed capacitor in shunt with a switched reactor which is controlled by two switches. The two switches are turned on and off in a complementary manner using a pulse-width modulation technique that allows the reactor to function as a continuously-variable inductor. The proposed compensator has several advantages 


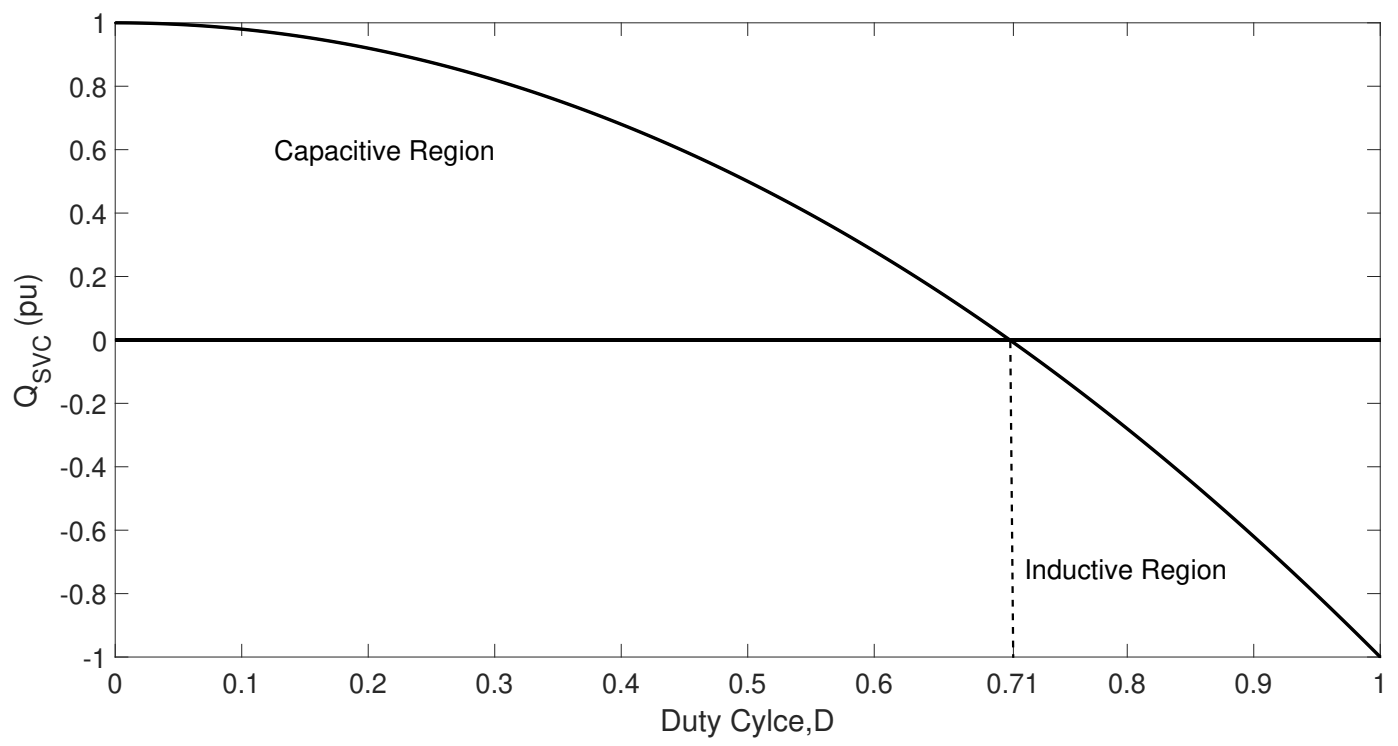

Figure 2.25: The reactive power provided by the PWM-based switched reactor with a fixed capacitor compensator.

compared to a conventional thyristor-based static var compensator (SVC), such as a quasisinusoidal inductor current, sub-cycle reactive power controllability, lower footprint for reactive components, and its realization as a single-phase device. 


\section{CHAPTER 3}

\section{BI-DIRECTIONAL SOLID STATE SWITCHES IN RESIDENTIAL STATIC VAR COMPENSATORS (RSVCS)}

The bi-directional switch (also known as a four-quadrant switch or 4Q switch or AC switch) is an essential part of the proposed Residential Static VAR Compensator. A bi-directional switch has the capability of conducting current in both directions, as well as blocking voltages of both polarities. The realization of these switches presents technical challenges in the implementation of current commutation techniques in direct AC switching. Research has been underway to fabricate a bi-directional switch on a single silicon die $[37,38]$. So far,

very few bi-directional switches are available in the power electronics market [39]. Therefore, discrete unidirectional switches are used to implement bi-directional switches. In this chapter, the realization of bi-directional switches is presented and the current commutation techniques from one phase to another phase through bi-directional is also discussed.

\subsection{Bi-Directional Switches Topologies}

Bi-directional switches are realized using discrete semiconductor devices. Figure 3.1 shows four possible topologies to realize bi-directional switches. In Figure 3.1a, a bi-directional switch has been created by two anti-parallel thyristors similar to the ones present in thyristorcontrolled reactors or thyristor-switched capacitors. In the switch-off case, i.e., when both thyristors are off, the terminal voltage appears across the thyristors. In the switch-on case, 
both thyristors are on and the current can pass in either direction. It is possible to control the direction of the current by controlling the switch-on conditions of the thyristors, i.e., by controlling the phase-angle at which the thyristors are triggered. This type of bi-directional switch requires two driving circuitry, one for each thyristor. The switch-on power losses for two anti-parallel thyristors depend on the voltage drop across the thyristor in the switch-on condition. Since only one device is active for conducting positive or negative cycles of current, the switch-on power losses are minimum. However, a drawback for such a topology is that, once the thyristor conducts, it does not turn off until the current through it becomes negative. The current stays zero until another gate trigger pulse is applied and the thyristor begins conducting again. This makes the operation of a bi-directional switch consisting of two anti-parallel thyristors very slow.

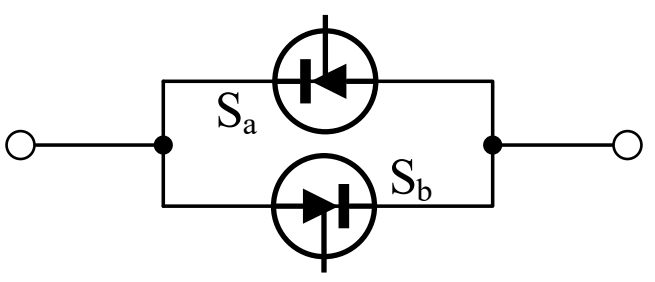

(a) Anti-parallel Thyristors

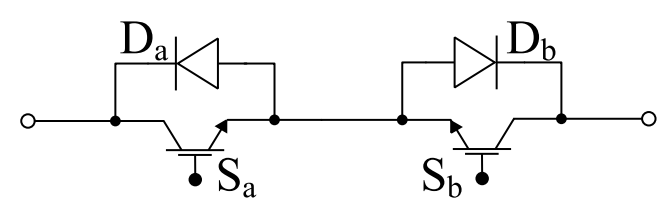

(c) Common-emitter

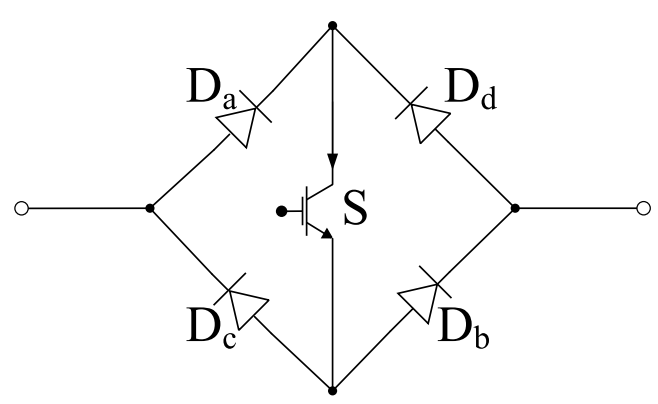

(b) Diode-bridge

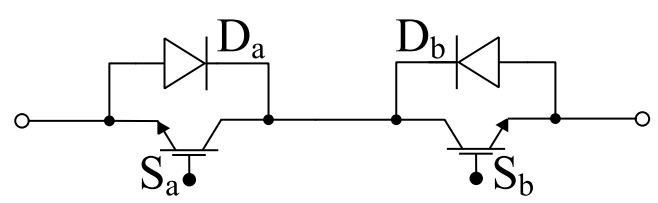

(d) Common-collector

Figure 3.1: Topologies of bi-directional switches.

The diode-bridge bi-directional switch arrangement, shown in Figure 3.1b, consists of a unidirectional IGBT at the center of a four diode-bridge arrangement [40]. The main advantage associated with a diode-bridge topology is that only one gate driving circuitry 
is required to control the flow of current. In the switch-off case, the IGBT is off and the terminal voltage of either polarity is applied to the IGBT through the diodes. For the positive direction of current, as shown in the figure, diodes $D_{a}$ and $D_{b}$ conduct through the IGBT. When the current direction changes, it is commuted through the opposite conducting diodes $D_{c}$ and $D_{d}$. Therefore, there are three devices involved in the conduction of the current, which contributes to greater switch-on conduction losses compared to two antiparallel thyristors. Also, it is impossible to control the direction of the current conduction. The current will conduct in either direction when the IGBT is switched on. The IGBT can be switched much faster than the thyristors, so high-speed switching is possible by using a diode-bridge bi-directional switch topology.

In Figure 3.1c, a bi-directional switch has been created by using two unidirectional IGBTs and two diodes $[41,42]$. In this topology, a conduction path for the current exists in both directions. Also, the diodes provide the reverse voltage blocking capability. In the switch-off case, both IGBTs are off and the terminal voltage appears across one of the IGBT switches, depending on the voltage polarity. In the switch-on case, the current is conducted by the IGBT and a diode, depending on the direction. For example, the current passes through $S_{a}, D_{b}$ or $S_{b}, D_{a}$. The two IGBTs can be used independently to control the current direction as well. The switch-on conduction losses are less than a diode-bridge bi-direction switch as only two devices are used for conduction. Moreover, faster switching is possible for the switches using such a bi-directional switching topology.

In Figure 3.1d, a bi-directional switch similar to the switch in Figure 3.1c is shown. The difference between the two topologies is based on the common terminal connection. The topology in Figure 3.1c is called the common-emitter bi-directional switch topology while the one in Figure 3.1d is called the common-collector topology. This dissimilarity in the topology does not cause any difference in the performance of two switches; only 
the number of the required isolated supplies for driving the IGBTs vary in both topologies. The main advantage of the common-emitter bi-directional switch topology is that the two IGBTs can be driven with respect to the same point, i.e., the common emitter, that can be considered as an isolated ground for the bi-directional switch (similar to the gate driving ground). However, for the common-collector only one power supply per input and output phase is required. For a single-phase circuit, two isolated power supplies are needed in both configurations.

Table 3.1 compares the characteristics of the bi-directional switches of the above mentioned bi-directional switches briefly. For the rest of this section and throughout the dissertation, the common-emitter bi-directional switch topology is used and the topology will be simply referred as a bi-directional switch.

Table 3.1: Comparison of different bi-directional switches topologies.

\begin{tabular}{|c|c|c|c|}
\hline $\begin{array}{l}\text { Bi-Directional } \\
\text { Switch Topology }\end{array}$ & $\begin{array}{c}\text { Total Number } \\
\text { of Devices }\end{array}$ & $\begin{array}{c}\text { Switch-on } \\
\text { Conduction Losses }\end{array}$ & Pros \& Cons \\
\hline $\begin{array}{l}\text { Anti-parallel } \\
\text { thyristor }\end{array}$ & 2 thyristors & 1 thyristor & $\begin{array}{l}\text { (+) Lower power loss. } \\
\text { (-) Conduction continues } \\
\text { until the current is zero. } \\
\text { (-) Slower operation. }\end{array}$ \\
\hline Diode-bridge & $\begin{array}{c}4 \text { Diodes \& } \\
\text { IGBT/MOSFET }\end{array}$ & $\begin{array}{c}2 \text { Diodes \& } \\
\text { IGBT/MOSFET }\end{array}$ & $\begin{array}{l}\text { (+) Faster operation. } \\
\text { (-) Current direction can } \\
\text { not be controlled. } \\
\text { (-) More power losses. }\end{array}$ \\
\hline $\begin{array}{l}\text { Common-emitter / } \\
\text { Common-collector }\end{array}$ & $\begin{array}{c}2 \text { Diodes \& } \\
2 \text { IGBT/MOSFET }\end{array}$ & $\begin{array}{c}\text { Diode \& } \\
\text { IGBT/MOSFET }\end{array}$ & $\begin{array}{l}\text { (+) Faster operation. } \\
\text { (+) Current direction can } \\
\text { be controlled. } \\
\text { (-) Moderate power loss. }\end{array}$ \\
\hline
\end{tabular}




\subsection{Current Commutation Problem Between Bi-directional Switches}

Current commutation between the bi-directional switches requires special attention and conventional commutation methods, as in the case of voltage source inverters, are not applicable. To understand this, consider a simplified single-phase RSVC circuit as shown in Figure 3.2. In the figure, SW1 and SW2 represent two bi-directional switches. Suppose that switch SW1 is on and SW2 is off, then the inductor current $i_{L}$ passes from SW1. If it is required to turn-on switch SW2 and turn-off switch SW1, then there are two possible solutions:

(i) turn-off switch SW1 and then turn-on SW2 (break-before-make)

(ii) turn-on switch SW2 and then turn-off SW1 (make-before-break).

For a break-before-make circuit, both switches will be open for a short interval (also known as the dead-time period). This will cause an interruption in the inductor current and a large voltage spike can appear across the switches potentially damaging the switches. Circuits using a break-before-make strategy normally use a voltage clamp circuit along with local snubber circuits to prevent damaging voltage spikes. For a make-before-break circuit, both switches will be closed for a short time. During this overlapping time, shortcircuit scenarios appear and large currents flow through the switches. This current surge can be destructive for the semiconductor devices and may lead to permanent damage to the switching $[43,44]$.

Therefore, the current commutation between the bi-directional switches requires specific control. The commutation requirements can be summarized as follows: 


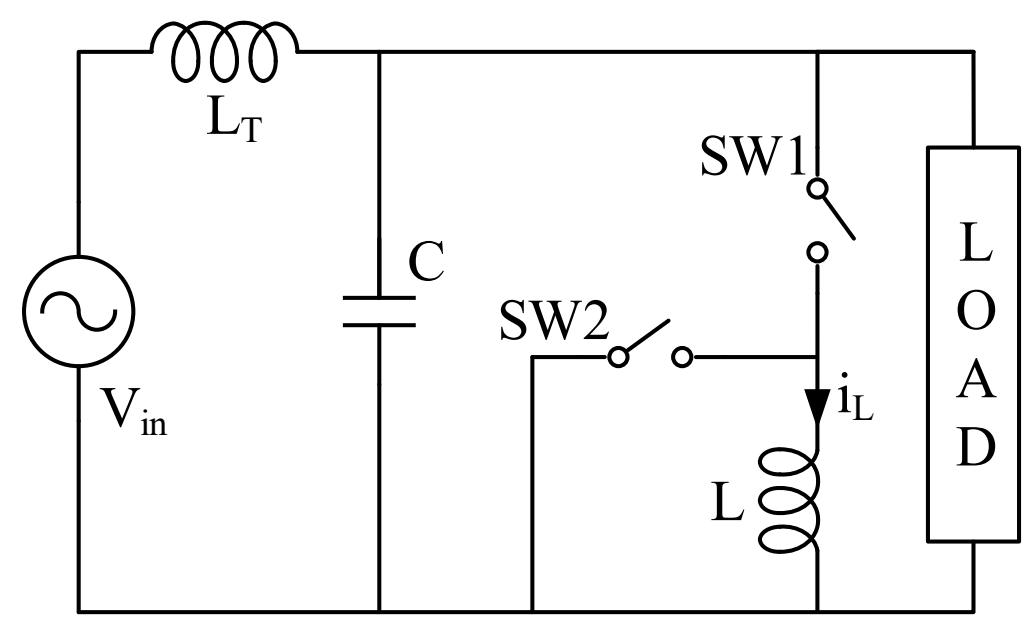

Figure 3.2: A simplified RSVC circuit to explain the problems with conventional switching methods.

1. The commutation from one switch to the other switch (or from one phase to the other) should not short-circuit the two phases. A short-circuit of the phases leads to large short-circuit currents which are fatal for the switching devices.

2. The commutation should not interrupt the inductive current. Interrupting the flow of inductive current leads to large voltage spikes.

The bi-directional switch having a topology as shown in Figure 3.1c can be used to commutate current from one phase to another phase based on the current direction or on the sign of the voltage across the commutating bi-directional switches [45-52]. These commutation strategies are explained in the following sections.

\subsection{Current Commutation Based on the Load Current}

A reliable method for current commutation between two bi-directional switches involves a four-step commutation strategy based on the direction of the load current (output current). 
The goal of this strategy is to strictly follow the two aforementioned rules for safe commutation. To explain this strategy, consider the single-phase circuit shown in Figure 3.3. In this circuit, the output load current $I_{o}$ is assumed to be positive if it flows from one of the input phases to the output phase. From Figure 3.3, it can be seen that the forward current, from the input phase to the output phase, flows through switches $S_{1 a}$ and $D_{1 a}$ or $S_{2 a}$ and $D_{2 a}$ and the backward current, from the output phase to the input phase, flows through switches $\mathrm{S}_{1 \mathrm{~b}}$ and $\mathrm{D}_{1 \mathrm{~b}}$ or $\mathrm{S}_{2 \mathrm{~b}}$ and $\mathrm{D}_{2 \mathrm{~b}}$.

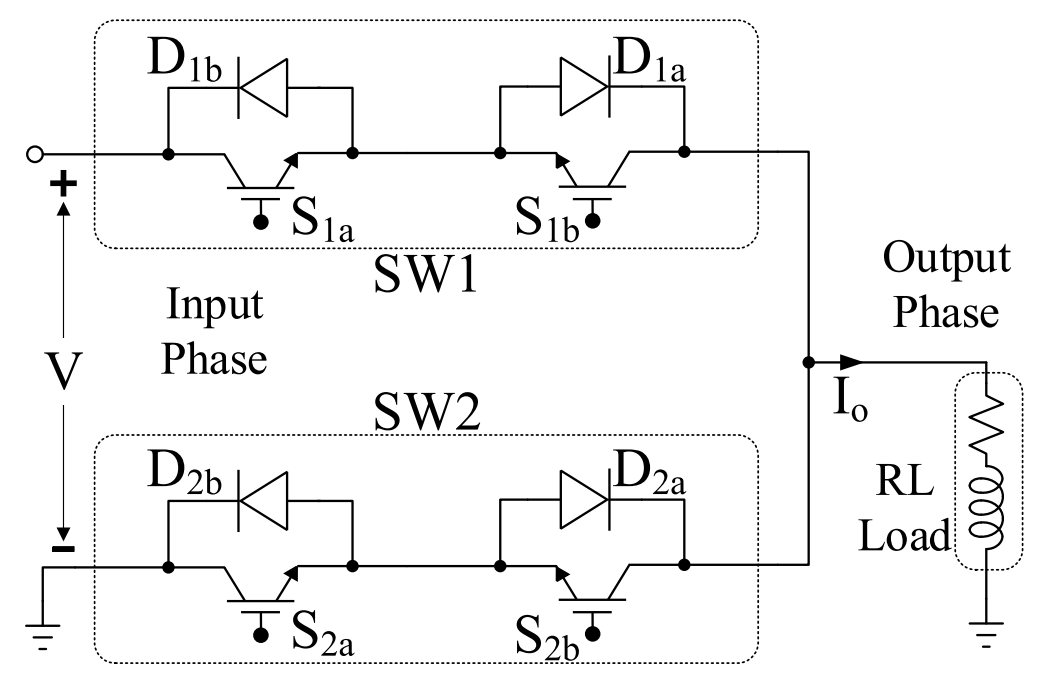

Figure 3.3: A circuit for understanding current commutation based on load current.

In the circuit, suppose that switch SW1 is on (or closed) and SW2 is off (or open). This commutation strategy assumes that when a switch is conducting, both the IGBT devices for the corresponding bi-directional switch are on. After a certain instant of time, SW1 is turning off and SW2 is turning on. Assuming that the output load current $\mathrm{I}_{\mathrm{o}}$ is flowing in the positive direction, the current commutation from the outgoing switch SW1 to the incoming switch SW2 based on the output current direction involves the following four steps:

1. the IGBT from the outgoing switch that is not conducting the output current is turned 
off. Since the current direction is positive, $S_{1 a}$ is used for conduction. So, $S_{1 b}$ is turned off;

2. the IGBT from the incoming switch that will conduct the output current is turned on. In this case, $\mathrm{S}_{2 \mathrm{a}}$ is turned on;

3. the IGBT from the outgoing switch that is conducting the output current is turned off. In this case, $\mathrm{S}_{1 \mathrm{a}}$ is turned off;

4. the IGBT from the incoming switch that will not conduct the output current is turned on. In this case, $S_{2 b}$ is turned on.

In case the output current is flowing in the opposite direction, i.e., from the output phase to the input phase, the commutation from outgoing switch SW1 to the incoming switch SW2 is performed in the following steps: (i) IGBT $\mathrm{S}_{1 \mathrm{a}}$ is turned off; (ii) IGBT $\mathrm{S}_{2 \mathrm{~b}}$ is turned on; (iii) IGBT $\mathrm{S}_{1 \mathrm{~b}}$ is turned off; (iv) IGBT $\mathrm{S}_{2 \mathrm{a}}$ is turned on.

A list of non-hazardous combinations of switching sequences can be achieved. These switching combinations are listed in Table 3.2. In Table 3.2, a logic-high or logic-one (1) means the IGBT is on or conducting whereas a logic-low or logic-zero (0) means that the IGBT is off or open. Any other combination of switching signals will violate either one of the two rules for safe-commutation. States 1 and 2 are called steady-states and States 3 through 8 are called transitional states. The commutation sequence of switches should start from a steady-state and end in another steady-state while going through three transitional states.

The commutation sequence can also be summarized in the state machine diagram shown in Figure 3.4. It should be noted that there is a built-in time delay, $t_{d}$, between each transitional state. This time delay should be greater than the sum of the maximum propagation 
Table 3.2: Non-hazardous combinations for current commutation based on the direction of load current.

\begin{tabular}{|c|c|c|c|c|c|}
\hline State & $\mathrm{S}_{1 \mathrm{a}}$ & $\mathrm{S}_{1 \mathrm{~b}}$ & $\mathrm{~S}_{2 \mathrm{a}}$ & $\mathrm{S}_{2 \mathrm{~b}}$ & $\begin{array}{c}\text { Output Current } \\
\text { Direction }\end{array}$ \\
\hline \hline 1 & 1 & 1 & 0 & 0 & +ve or -ve \\
\hline 2 & 0 & 0 & 1 & 1 & +ve or -ve \\
\hline 3 & 1 & 0 & 0 & 0 & +ve \\
\hline 4 & 0 & 0 & 1 & 0 & +ve \\
\hline 5 & 1 & 0 & 1 & 0 & +ve \\
\hline 6 & 0 & 1 & 0 & 0 & -ve \\
\hline 7 & 0 & 0 & 0 & 1 & -ve \\
\hline 8 & 0 & 1 & 0 & 1 & -ve \\
\hline
\end{tabular}

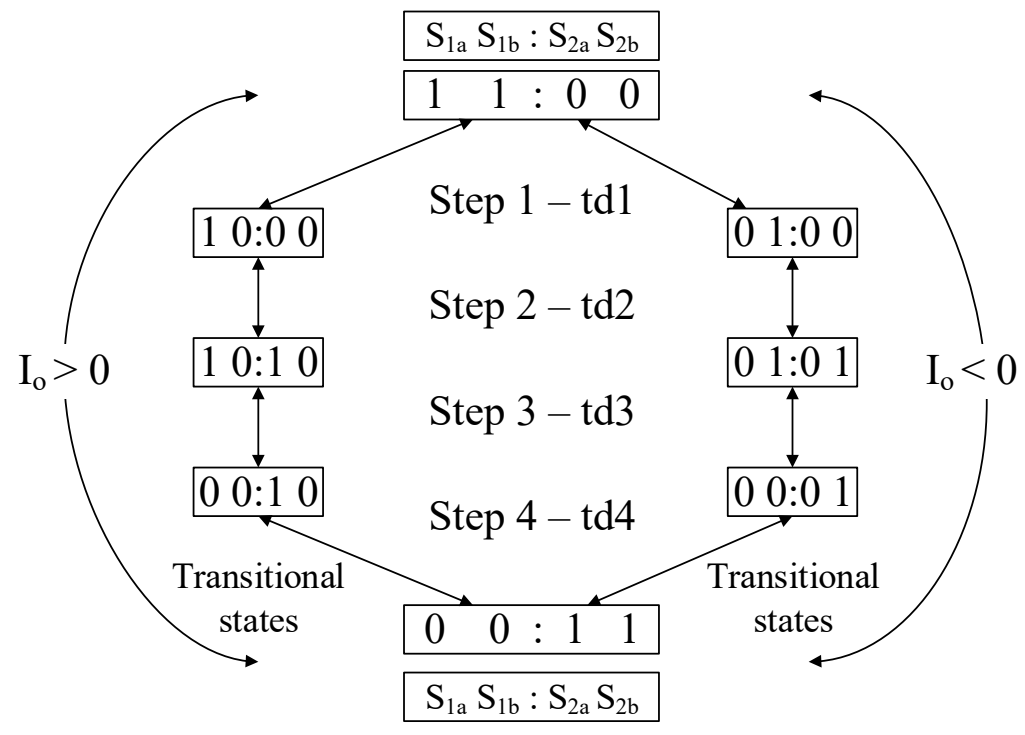

Steady States

Figure 3.4: Four-step switching state machine for two bi-directional switches based on output load current.

delays required by the IGBT gating signals, the rise-time, and the fall-time. In the figure, the positive direction of the current is shown as $I_{0}>0$ and the negative direction of the current is shown as $\mathrm{I}_{\mathrm{o}}<0$. 


\subsection{Current Commutation Based on the Voltage Across the Bi-directional Switches}

This section will describe another method for current commutation for bi-directional switches. This commutation technique is based on the sign of the voltage across the bi-directional switches which are involved in the commutation. As with the current commutation using the load current, this strategy assumes that when an output phase is connected to the input phase, both the IGBT devices for the corresponding bi-directional switch are on. Consider again the circuit shown in Figure 3.3. For the first case consider the voltage difference $\mathrm{V}$ across SW1 and SW2 is positive. The positive and negative current commutation from SW1 to SW2 involves the following steps:

1. $\mathrm{S}_{2 \mathrm{a}}$ is turned on. Since voltage $\mathrm{V}$ is positive, $\mathrm{D}_{2 \mathrm{a}}$ is reverse biased and there is no short-circuit from the input phase to the ground. The current keeps flowing through SW1 as shown in Figure 3.5b for positive current and Figure 3.6b for negative current;

2. $\mathrm{S}_{1 \mathrm{a}}$ is turned off. If the direction of the current is positive then the current is commutated from SW1 to SW2 at this step as shown in Figure 3.5c. However, if the direction is negative then the current keeps flowing through SW1 as shown in Figure 3.6c;

3. $S_{2 b}$ is turned on. Since $S_{1 a}$ is turned off in the previous step, there is no risk of short circuiting. There is a path of current available for both directions as shown in Figure 3.5d for positive direction and in Figure 3.6d for a negative direction through SW2;

4. Finally, $S_{1 b}$ is turned off. At this stage, the SW1 is completely off and SW2 is on. 

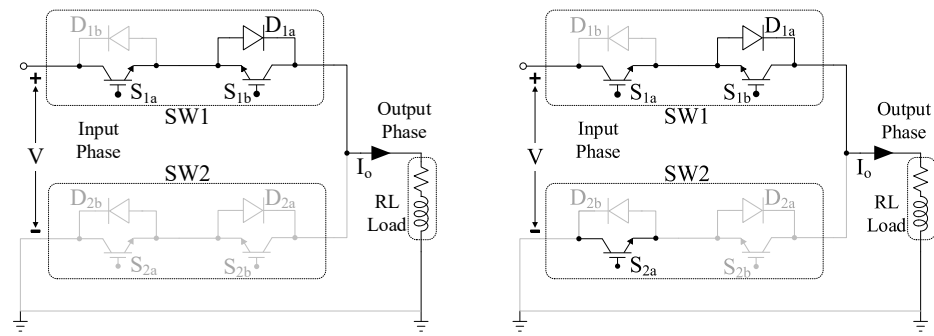

(a) $\mathrm{S}_{1 \mathrm{a}}=1 ; \mathrm{S}_{1 \mathrm{~b}}=1 ; \mathrm{S}_{2 \mathrm{a}}=0 ; \mathrm{S}_{2 \mathrm{~b}}=0$ (b) $\mathrm{S}_{1 \mathrm{a}}=1 ; \mathrm{S}_{1 \mathrm{~b}}=1 ; \mathrm{S}_{2 \mathrm{a}}=1 ; \mathrm{S}_{2 \mathrm{~b}}=0$

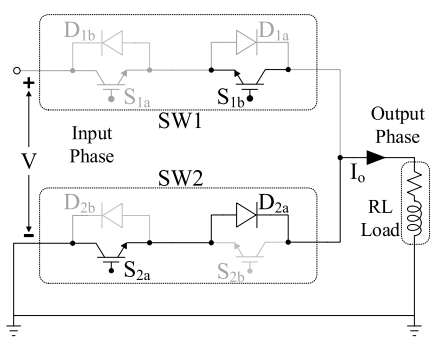

(c) $\mathrm{S}_{1 \mathrm{a}}=0 ; \mathrm{S}_{1 \mathrm{~b}}=1 ; \mathrm{S}_{2 \mathrm{a}}=1 ; \mathrm{S}_{2 \mathrm{~b}}=0$

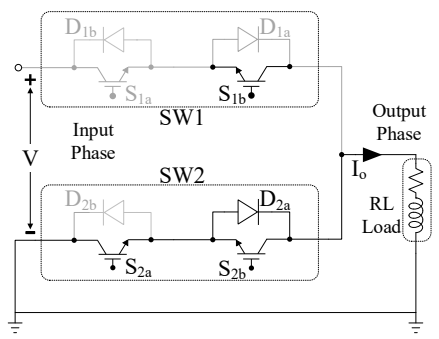

(d) $\mathrm{S}_{1 \mathrm{a}}=0 ; \mathrm{S}_{1 \mathrm{~b}}=1 ; \mathrm{S}_{2 \mathrm{a}}=1 ; \mathrm{S}_{2 \mathrm{~b}}=1$

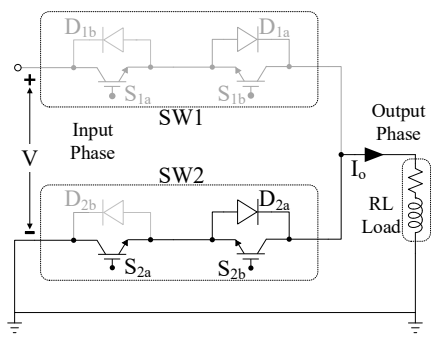

(e) $S_{1 a}=0 ; S_{1 b}=0 ; S_{2 a}=1 ; S_{2 b}=1$

Figure 3.5: Positive voltage-based current commutation from SW1 to SW2 when the current direction is positive.
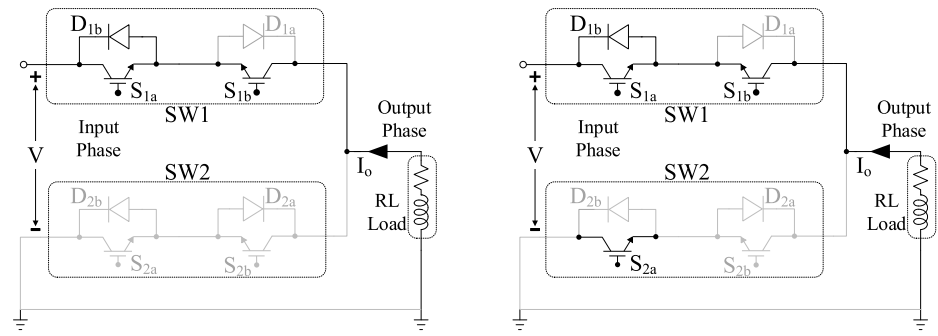

(a) $\mathrm{S}_{1 \mathrm{a}}=1 ; \mathrm{S}_{1 \mathrm{~b}}=1 ; \mathrm{S}_{2 \mathrm{a}}=0 ; \mathrm{S}_{2 \mathrm{~b}}=0$

(b) $\mathrm{S}_{1 \mathrm{a}}=1 ; \mathrm{S}_{1 \mathrm{~b}}=1 ; \mathrm{S}_{2 \mathrm{a}}=1 ; \mathrm{S}_{2 \mathrm{~b}}=0$
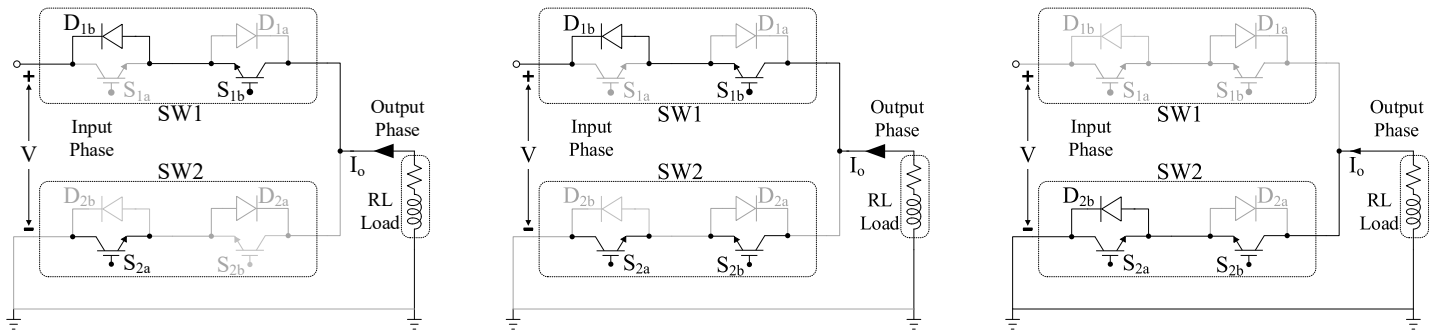

(c) $S_{1 a}=0 ; S_{1 b}=1 ; S_{2 a}=1 ; S_{2 b}=0$

(d) $\mathrm{S}_{1 \mathrm{a}}=0 ; \mathrm{S}_{1 \mathrm{~b}}=1 ; \mathrm{S}_{2 \mathrm{a}}=1 ; \mathrm{S}_{2 \mathrm{~b}}=1$

(e) $S_{1 a}=0 ; S_{1 b}=0 ; S_{2 a}=1 ; S_{2 b}=1$

Figure 3.6: Positive voltage-based current commutation from SW1 to SW2 when the current direction is negative. 
In the case when $\mathrm{V}$ is negative, the current commutation from SW1 to SW2 involves the following steps: (i) $S_{2 b}$ is turned on; (ii) $S_{1 b}$ is turned off; (iii) $S_{2 a}$ is turned on; (iv) $S_{1 a}$ is turned off. The commutation of the positive current, from the input phase to output phase is shown in Figure 3.5 and Figure 3.6 shows the commutation of negative current from SW1 to $\mathrm{SW} 2$ when voltage $\mathrm{V}$ is positive.

These switching signals are presented in a state machine diagram in Figure 3.7. As in the case of load current based current commutation, state machines states where only both IGBTs for the bi-directional switch are on, are called steady states and the remaining states are called transitional states. A small time delay between each transition is required to account for the propagation delays, the rise time and the fall time in the gating signals for the IGBTs.

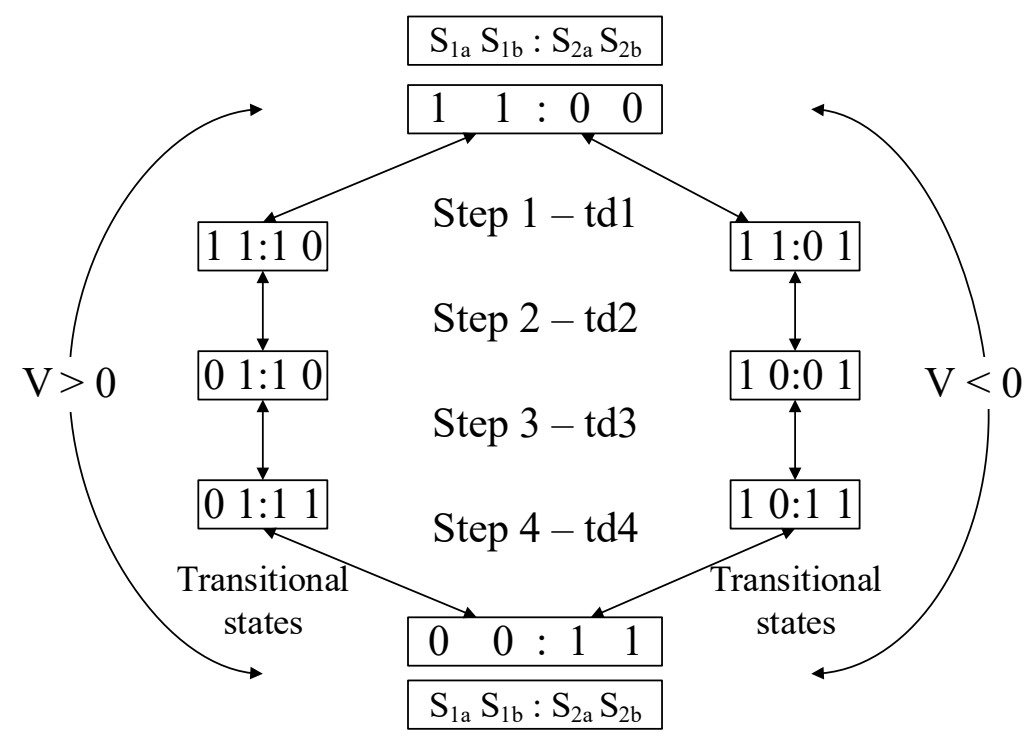

Steady States

Figure 3.7: Four-step switching state machine for two bi-directional switches based on the voltage polarity across the bi-directional switches. 


\subsection{Commutation of Current Between Bi-Directional Switches in the Residential Static VAR Compensator}

For the bi-directional switches in the RSVC, the voltage-based (voltage across the bidirectional switches) current commutation is chosen over the load current commutation. For a single-phase voltage, the bi-directional switch commutation becomes relatively simpler as it only involves detecting the sign of the phase-line with respect to the neutral point. For a balanced three-phase system, neutral is often grounded (zero potential), thus a circuitry is required to detect the polarity of the single-phase to which the RSVC is connected. Of course, if the measured voltage is wrong or the voltage sign changes during the transitional state, a short-circuit may occur. Therefore, to safely commutate from one switch to another, a protection mechanism can be adopted.

For the RSVC, this safety mechanism is adopted by halting the bi-directional switching state machine near the zero-crossing. For a sinusoidal bus voltage waveform, the current commutation using the bi-directional switching state machine can be carried out normally. This region is termed as the "Non-Critical Region" for current commutation. The "Critical Region" occurs near the zero-crossing, when the phase-to-neutral voltage becomes nearly equal to zero. The state machine is halted, and the commutation sequence is not executed until the input voltage has significantly increased (or decreased) above (or below) zero. It is important to stop the switching sequence at one of the two steady states. For the RSVC, the state machine is halted at a steady state of $[00: 11]$, i.e., when SW2 is closed and SW1 is open, during the critical region. The effect of a halt of the commutation sequence on the output voltage and the output current is not significant, as long as the "Critical Region" is kept small. However, due to the halt in the commutation, the inductor current waveform will differ from an ideal sinusoidal shape as explained in detail in the following chapter. 


\section{CHAPTER 4}

\section{PERFORMANCE ANALYSIS AND SIMULATION RESULTS OF THE RESIDENTIAL STATIC VAR COMPENSATOR}

The goal of the Residential Static VAR Compensator (RSVC) is adjusting and regulating the amplitude of output voltage at the distribution level. The operating principles of the single-phase RSVC have been shown in Chapter 2. This chapter focuses on the open-loop design and simulation of the RSVC using Matlab. The closed-loop controller design for voltage regulation is also presented in this chapter. The main responsibility of the voltage regulation controller in the closed-loop RSVC system is to track the output load voltage amplitude with respect to the reference voltage. A secondary closed-loop power regulation controller design is also presented. The objective of the power regulation loop is to find an optimal reference voltage at which the minimum power is consumed. The following sections will systematically present a complete RSVC design and the simulation results will show the accuracy of the open-loop and the closed-loop designs.

\subsection{RSVC Open-Loop Design Using Matlab/Simulink}

The discussion in this section focuses on the RSVC circuit shown in Figure 4.1. The reactive power generated or absorbed by the RSVC depends on the sizing of the fixed capacitor $\left(C_{S V C}\right)$ and switched inductor $\left(L_{S V C}\right)$. In general, the RSVC is a scalable device. It means that the reactive power components (the fixed capacitor and switched inductor) 
sizing depends on the voltage regulation requirements for different types of loads, i.e., individual customers in a distribution feeder, a specific building in a distribution feeder or a certain industrial or residential load.

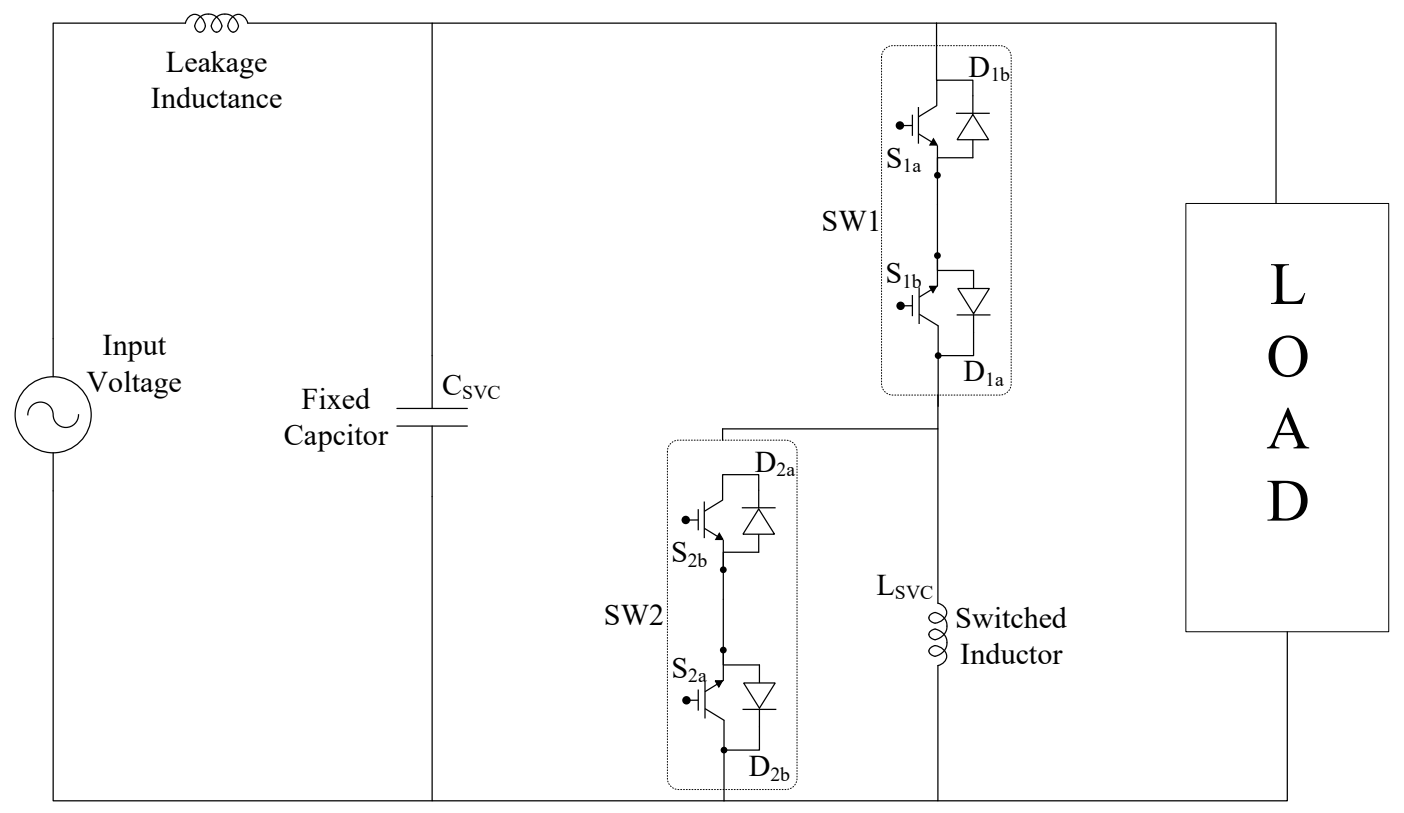

Figure 4.1: Circuit diagram of the Residential Static VAR Compensator.

\subsubsection{Design of the Four-Step Current Commutation State Machine Based on the Sign of Voltage Across the Bi-Directional Switches}

A state machine is developed by using the Simulink Stateflow toolbox which generates the gating signals for the bi-directional switches. The bi-directional switches are used to control the PWM-based switching of the reactor. Figure 4.2 shows the top-level design for the state machine. The state machine takes as input the output (load) voltage (voltage across the two bi-directional switches), duty-cycle $D$ and an enable signal $E n$ which enables (1) or disables (0) the state machine. The state machine produces four gating signals for the bi-directional switches, namely SW_1A, SW_1B, SW_2A and, SW_2B. 


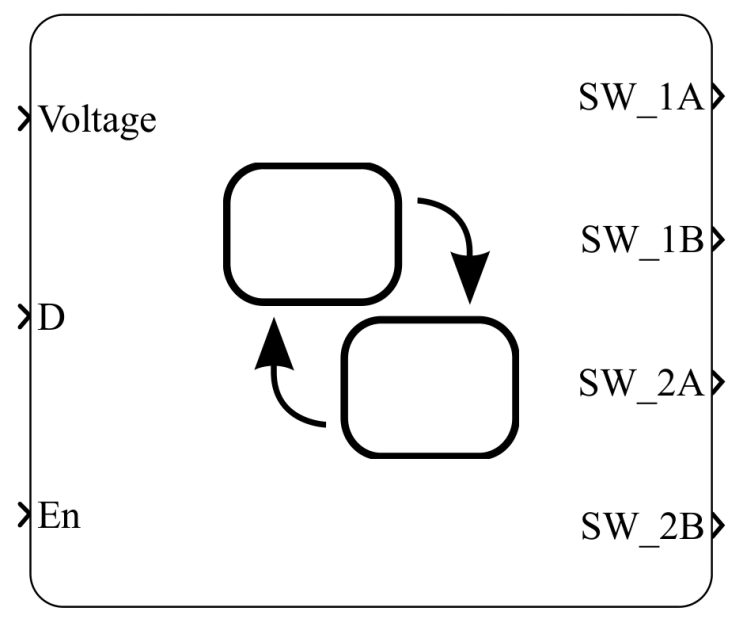

Figure 4.2: Top-level block diagram for the state machine producing gating signals for the bi-directional switches.

Figure 4.3 shows a detailed overview for generating the four gating signals using the Stateflow toolbox in Simulink. Each block in the figure represents a state of the state machine. The first line in each state (block) represents the unique name of that state. States State_1100 and State_0011 are called the steady states, whereas states State_1110, State_0110, State_0111, State_1011, State_1001 and State_1101 are called the transitional states. The state machine switches between steady states by passing through the transitional states.

When the enable switch En is off (0), the state machine is in the waiting state of State_0011. This means that the gating pulses for switch SW1, i.e., SW_1A and SW_1B are 0 and the gating pulses for switch SW2, i.e., SW_2A and SW_2B are 1. When the enable switch $E n$ is on, the state machine is ready for operation. State_0011 monitors the voltage across the bi-directional switches. Based on the polarity of the output voltage, the state machine starts transitioning to the next steady state, i.e., State_1100. The function sort_direction performs two operations: (i) it decides the direction of the state machine 


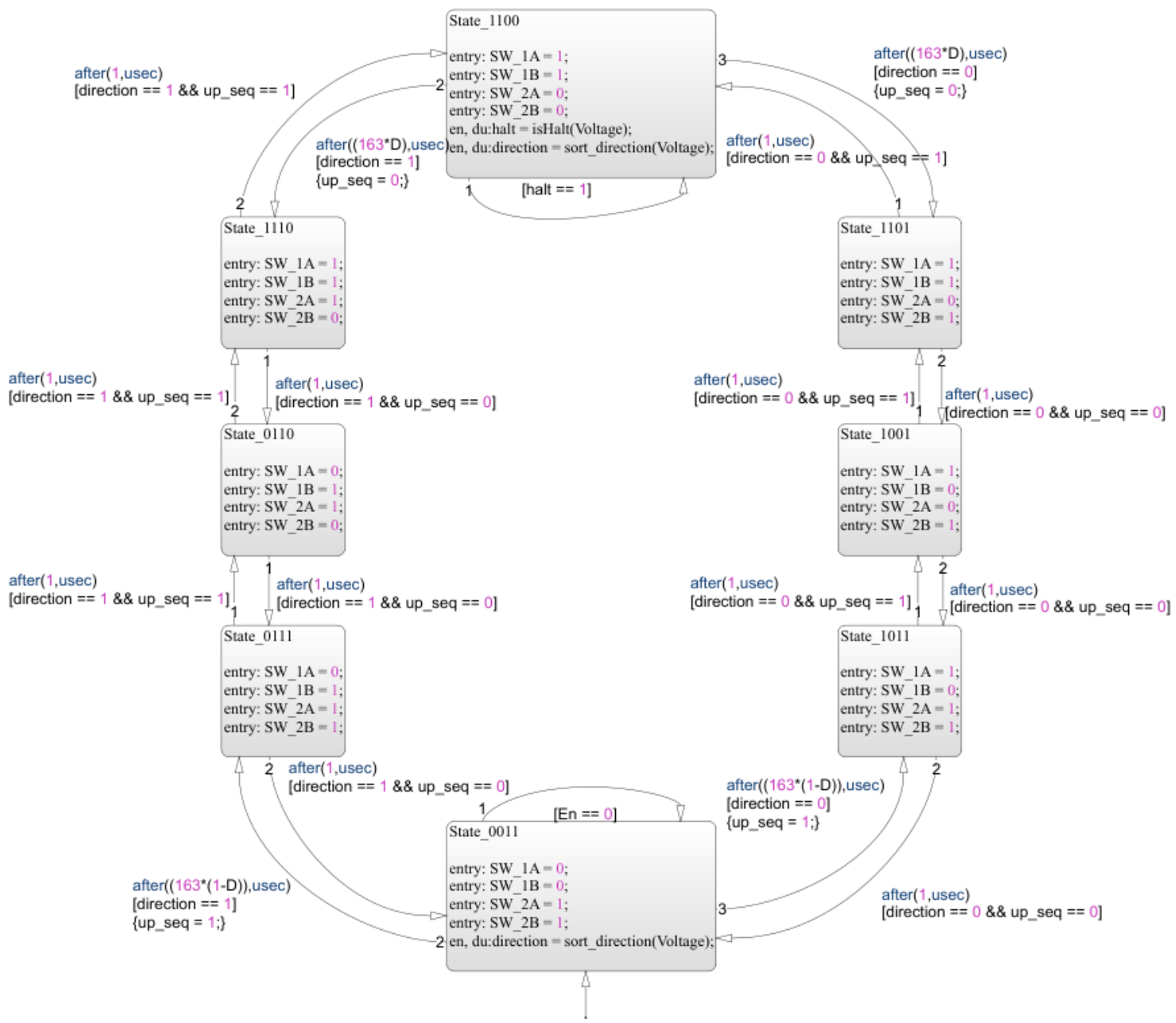

Figure 4.3: A complete state machine for producing gating signals SW_1A, SW_1B, SW_2A and SW_2B based on the output load voltage. The states (blocks) in the state machine represents the output of the state machine for each state.

based on the polarity of the voltage across the bi-directional switches (ii) and it makes sure that the state machine does not start the commutation near the zero-crossing when the enable switch $E n$ is turned on. If the voltage is close to zero, the commutation is halted to avoid false detection of the voltage polarity, especially when there is noise in the system. The state machine transitions to the first transitional state after completing $(1-D) \times t p_{s w}$ where $t p_{s w}$ represents the time period of the switching frequency and $D$ represents the duty 
cycle of the SW1. In other words, $D$ specifies the duration of the switching frequency for which the State_1100 (SW1) will remain active. A small delay time of $1 \mu$ sec between each transition is required to account for the propagation delays and the rise and fall times in the gating signals for the IGBTs. During the normal operation of the state machine, the state machine halts in State_0011 based on the output of the ishalt function when the voltage across the bi-directional switches is near the zero-crossing.

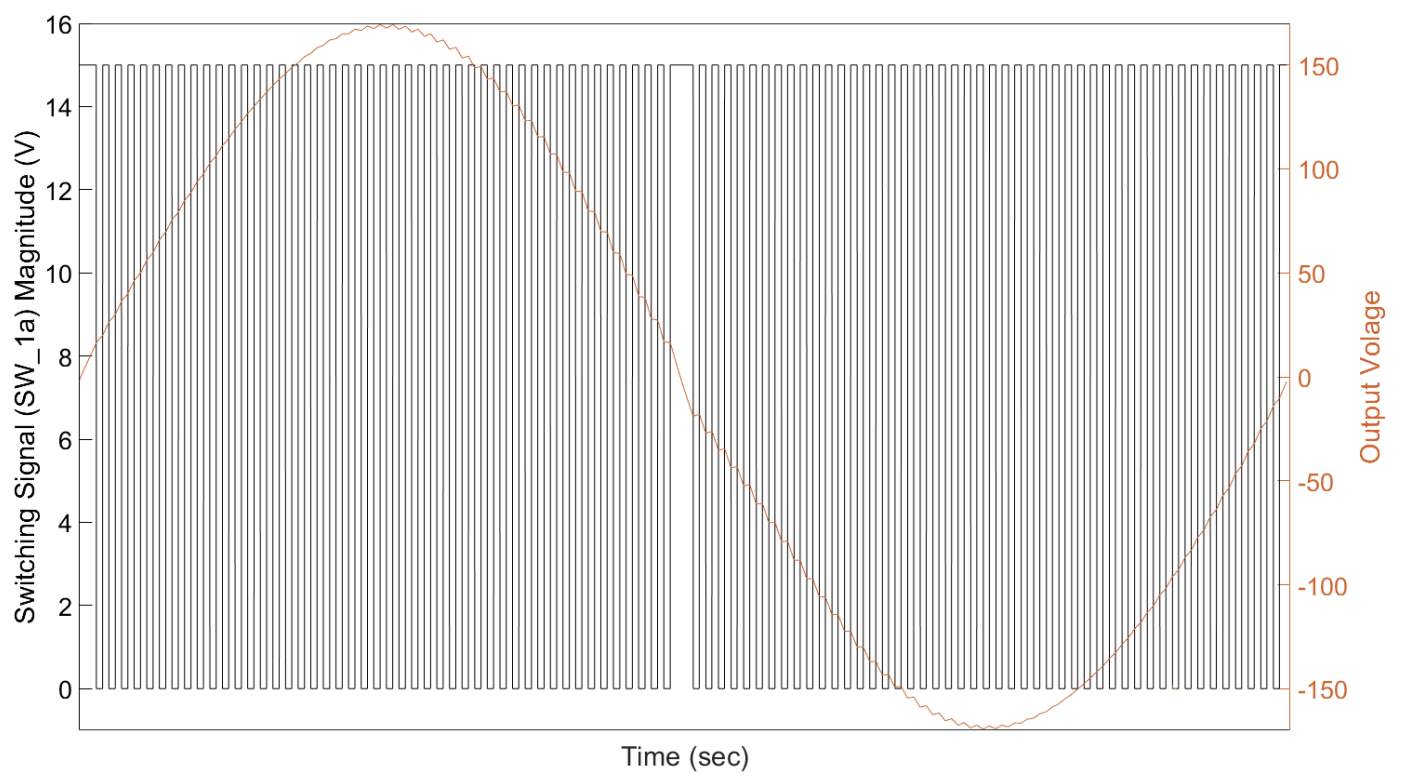

Figure 4.4: Output of the switch SW_1A from the state-machine for a complete cycle of the output voltage.

The overall output of the state machine with a switching signal of SW_1A and the output voltage (which is also the voltage across the bi-directional switches) is shown in Figure 4.4. It can be seen that the commutation sequence halts during the zero-crossing of the voltage. In Figure 4.5, the gating signals are shown for current commutation from SW1 to SW2 based on the amplitude of the voltage in the case of the output (load) voltage $V_{\text {out }}>0$. Whereas, the Figure 4.6 shows the gating signals for current commutation when $V_{\text {out }}<0$. 

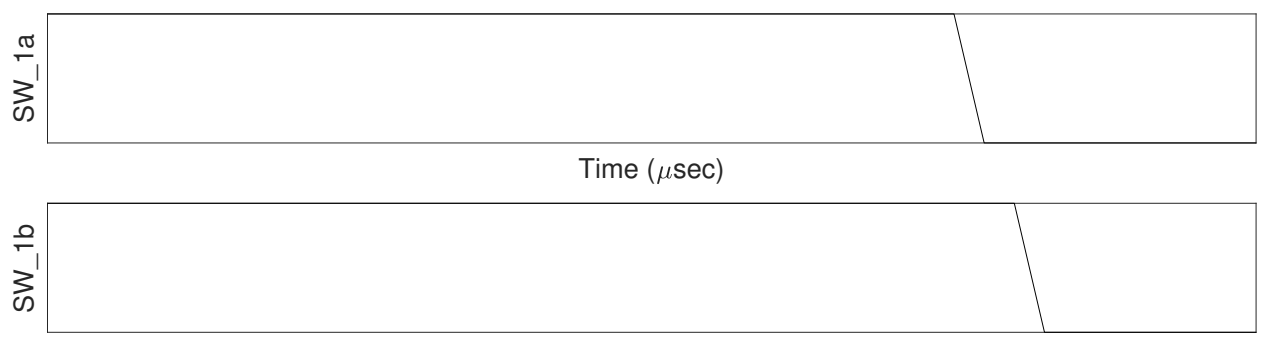

Time $(\mu \mathrm{sec})$
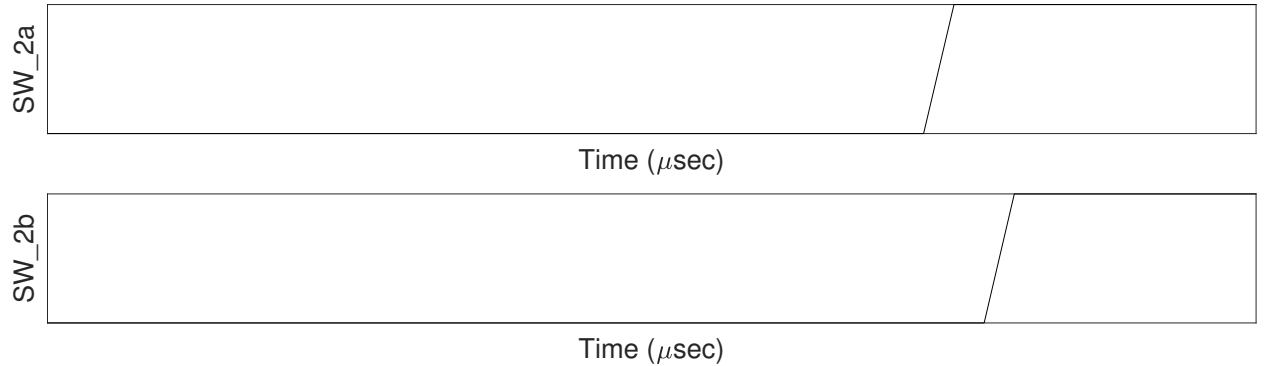

Figure 4.5: The gating signals for current commutation from SW1 to SW2 based on the amplitude of voltage when output voltage $V_{\text {out }}>0$.
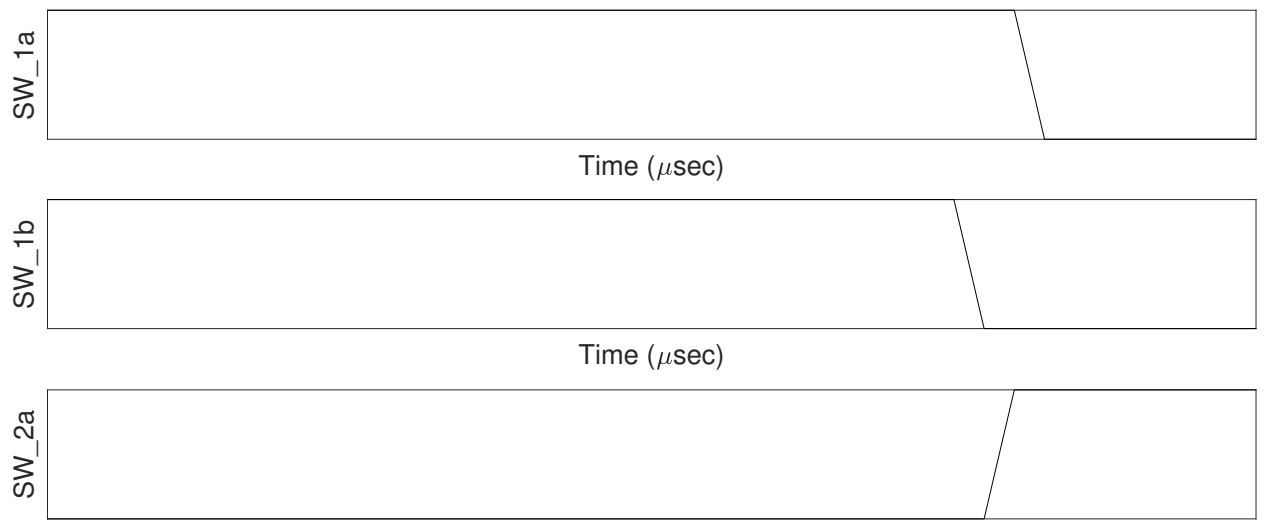

Time $(\mu \mathrm{sec})$

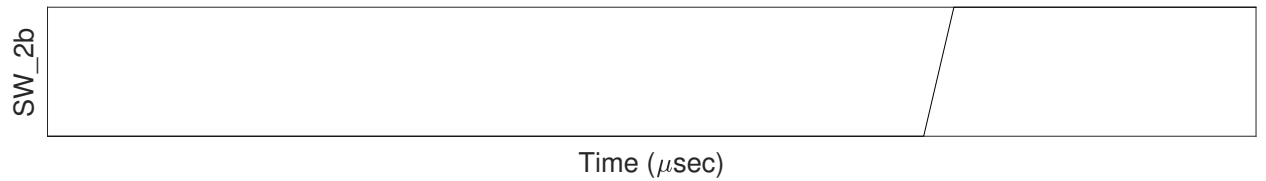

Figure 4.6: The gating signals for current commutation from SW1 to SW2 based on the amplitude of voltage when output voltage $V_{\text {out }}<0$. 


\subsubsection{Design of the Residential Static VAR Compensator (RSVC)}

The generation of the switching pulses from the state machine, as described in the previous section, is the heart of the proposed Residential Static VAR Compensator (RSVC). Figure 4.7 presents the complete model of the RSVC used in the simulation. To model the service transformer, a power system block is used in Simulink. The leakage reactance for the service transformer is assumed to be $10 \%$ of its rated nameplate capacity. The bi-directional switches are modelled using the common-emitter topology of two unidirectional IGBT with anti-parallel diodes. To simulate the actual characteristics of the bi-directional switches, a forward voltage $\left(V_{f}\right)$ drop of $1 \mathrm{~V}$ is assumed. Figure 4.8 shows the Simulink model of the bi-directional switches used for the simulation.

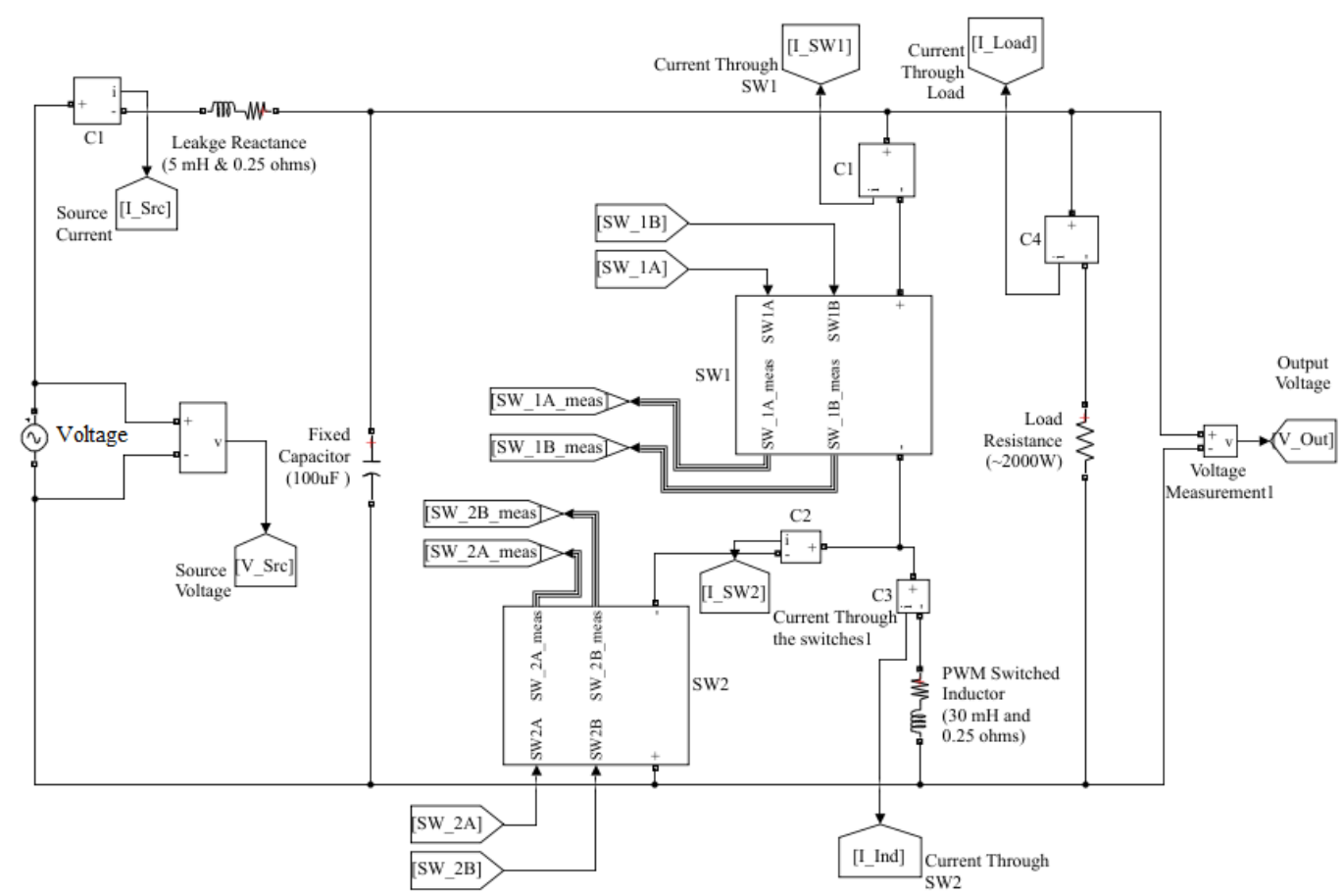

Figure 4.7: The RSVC Simulink model with a fixed capacitor and a switched inductor having bi-directional switches. 


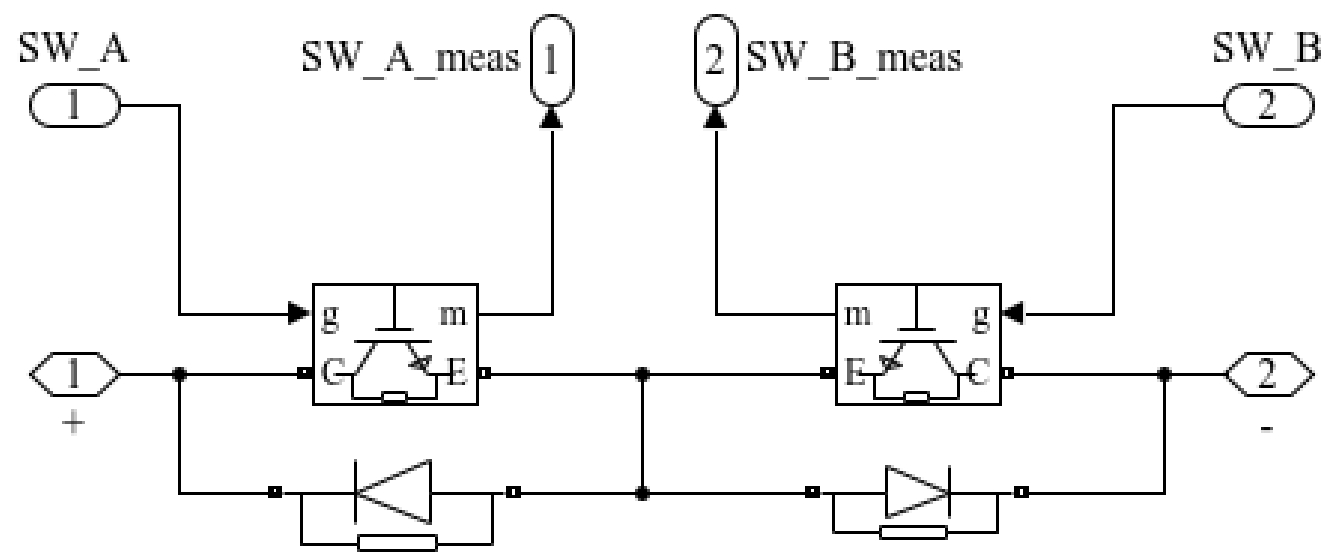

Figure 4.8: Bi-directional switches model in Simulink.

Other conditions and parameters used in the simulation circuit are as follows:

- The input voltage is sinusoidal with an RMS value of $120 \mathrm{~V}$ and a frequency of 60 $\mathrm{Hz}$.

- The leakage reactance, $X_{T}$, of the service transformer is set to $1.885 \Omega$ at $120 \mathrm{~V}$.

- The fixed capacitor $\left(C_{S V C}\right)$ and switched inductor $\left(L_{S V C}\right)$ are set to $100 \mu \mathrm{F}$ and $30 \mathrm{mH}$ respectively. The reactive power, at $120 \mathrm{~V}$, supplied by the fixed capacitor $\left(Q_{C}\right)=543$ VAR and the reactive power absorbed by switched inductor when it is fully on i.e., when duty cycle is $D=1$ is given as $\left.Q_{L}(D)\right|_{D=1}=1270$ VAR.

- A purely resistive load of $2000 \mathrm{~W}$ is used for the simulations. 


\subsection{RSVC Open-Loop Simulation and Performance Analysis}

\subsubsection{Voltage Waveforms}

The successful operation of the proposed SVC topology depends on the safe operation of the bi-directional switches. The safe operation of bi-directional switches ensures that (i) there is no short-circuiting of the two input phases, (ii) and the inductor (reactor) current remains continuous. The state machine developed in the previous section follows a specific commutation pattern that avoids hazardous operational conditions. Figure 4.9 shows the simulation results of the input (source) voltage and the output (load) voltage. These voltage waveforms are obtained at a duty cycle of $D=0.5$. It can be seen that the input and output current remains sinusoidal without any short-circuit during the operation.
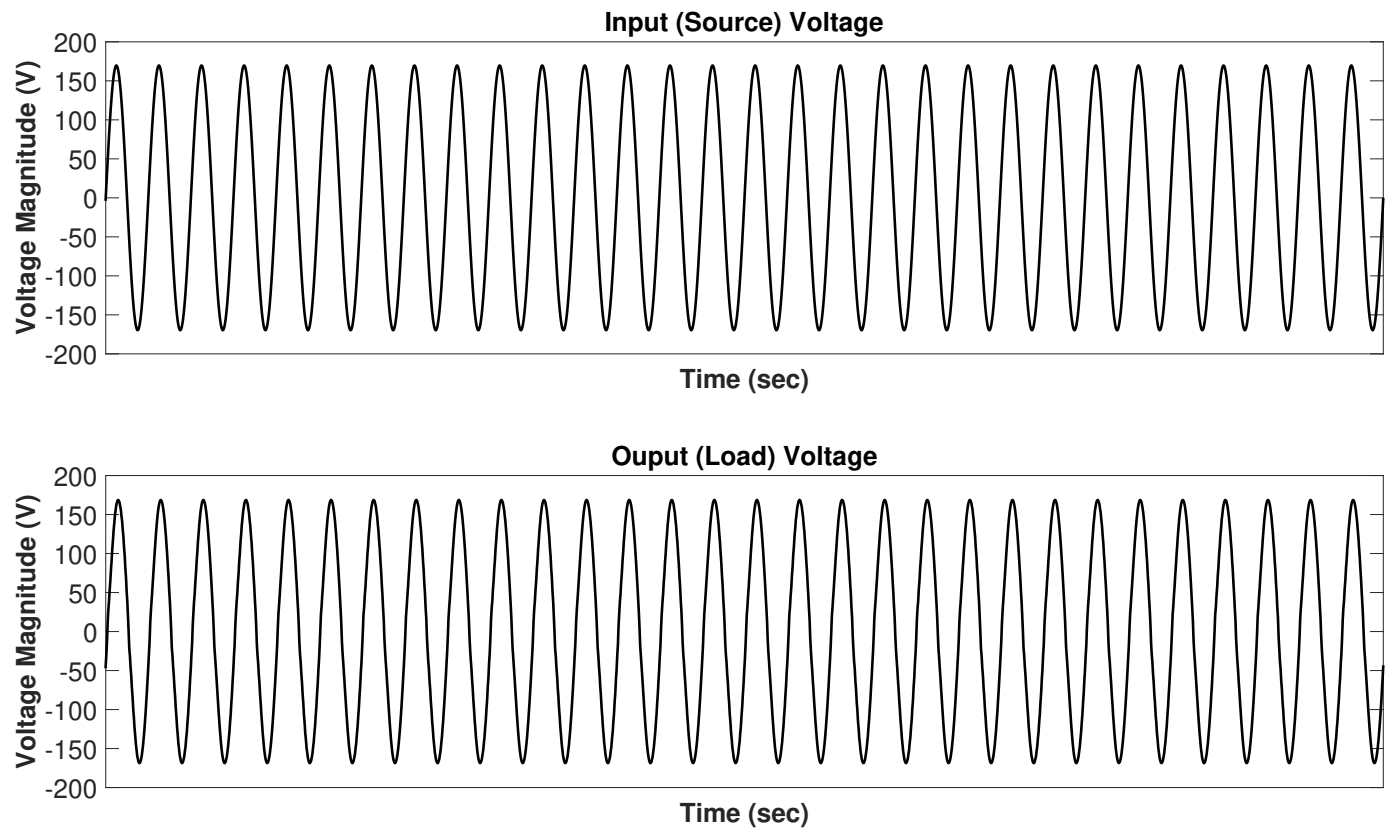

Figure 4.9: Input (source) and output (load) voltage waveforms at duty cycle $\mathrm{D}=0.5$. 


\subsubsection{Current Waveforms}

Figure 4.10 shows the input (source), output (load) and inductor current waveforms when the duty cycle $D=0.5$. The inductor current is continuous, as shown in the third plot of Figure 4.10, as opposed to the inductor current waveform in a thyristor-controlled reactor. By using a pulse-width modulation for turning on and off the two switches in a complementary manner, the inductor current becomes quasi-sinusoidal. This technique does not require the harmonic filtering which is an essential part of a conventional SVC. The input (source) current is shown in the first plot of Figure 4.10. Because the inductor current remains nearly sinusoidal, the PWM-based RSVC draws a sinusoidal current from the source and therefore does not introduce low-order harmonics into the power system.

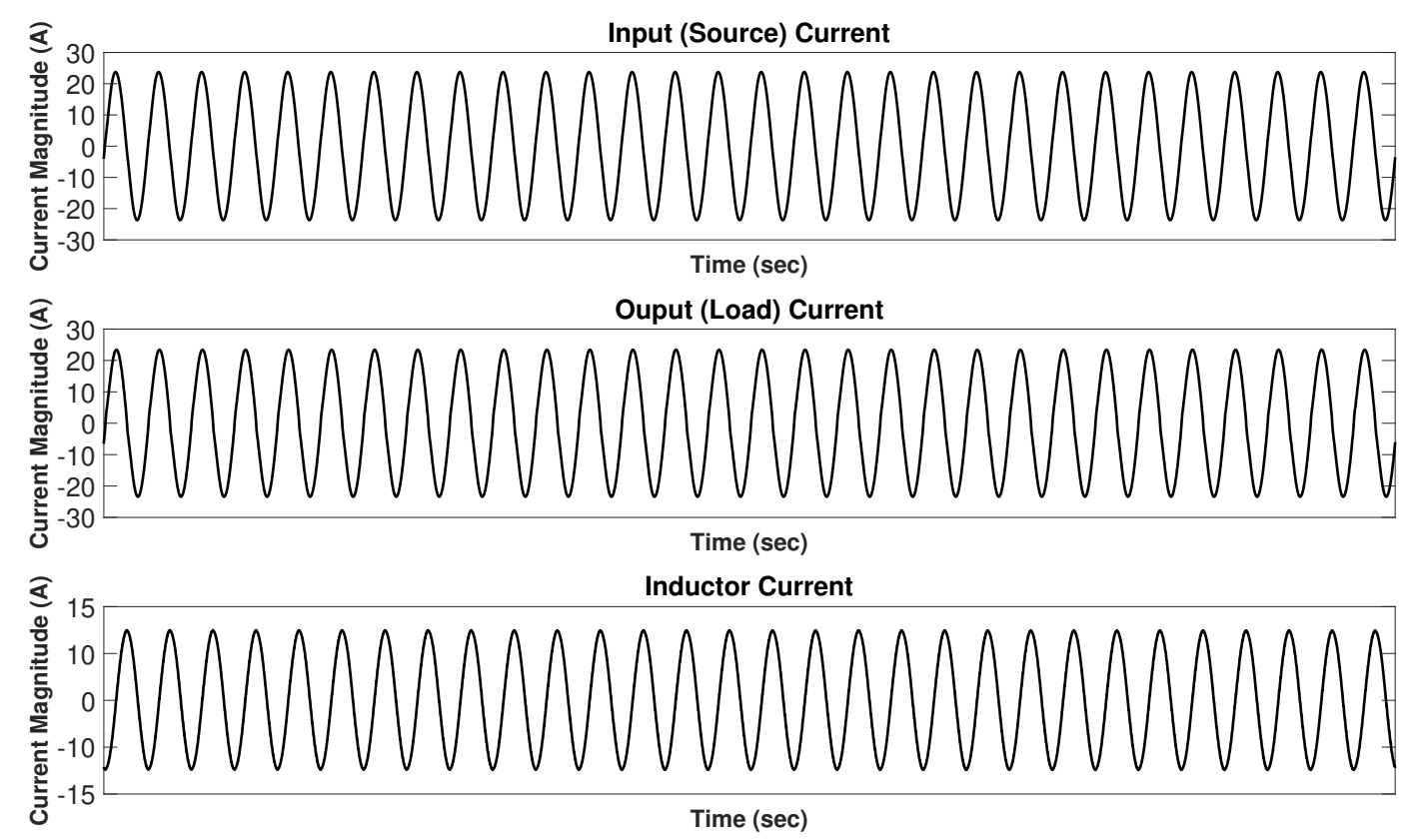

Figure 4.10: Input (source), output (load) and inductor current waveforms at duty cycle D $=0.5$. 


\subsection{Performance Analysis of Open-Loop RSVC}

After plotting the current and voltage waveforems in the previous sections, the following sections will cover the performance of the open-loop RSVC.

\subsubsection{Output (load) Voltage Variation With Duty Cycle}

Figure 4.11 shows the variation in the output (load) voltage with the variation in the duty cycle. The simulation was carried out with an ideal input voltage of $120 \mathrm{Vrms}$ at $60 \mathrm{~Hz}$ frequency. When the duty cycle is 0 , meaning the top switch connecting the reactor to the power circuit is off, the output voltage is at $123.5 \mathrm{Vrms}$. The increase in the voltage is due to the presence of a fixed capacitor in the circuit. When the duty cycle starts to increase, the inductor is connected to the power circuit, depending on the turn-on time specified by the duty cycle. As the duty circle reaches 1 , the inductor is connected for the complete switching period. It can be seen that the output voltage decreases with the duty cycle. In general, for the proposed RSVC, the output voltage decreases linearly with the square of the duty cycle.

By changing the duty cycle, it is possible to control the output voltage. As shown in the figure, when the duty cycle reaches 0.72 , the output voltage is equal to $114 \mathrm{Vrms}$, provided that the input voltage is at $120 \mathrm{Vrms}$. The output voltage of $114 \mathrm{Vrms}$ is the minimum allowable voltage specified by the ANSI C84.1 standard.

\subsubsection{Reactive Power Variation With Duty Cycle}

Figure 4.12 shows the variation of the net reactive power supplied by the RSVC with a change in the duty cycle. The net reactive power provided by the RSVC is calculated by the following equation, 


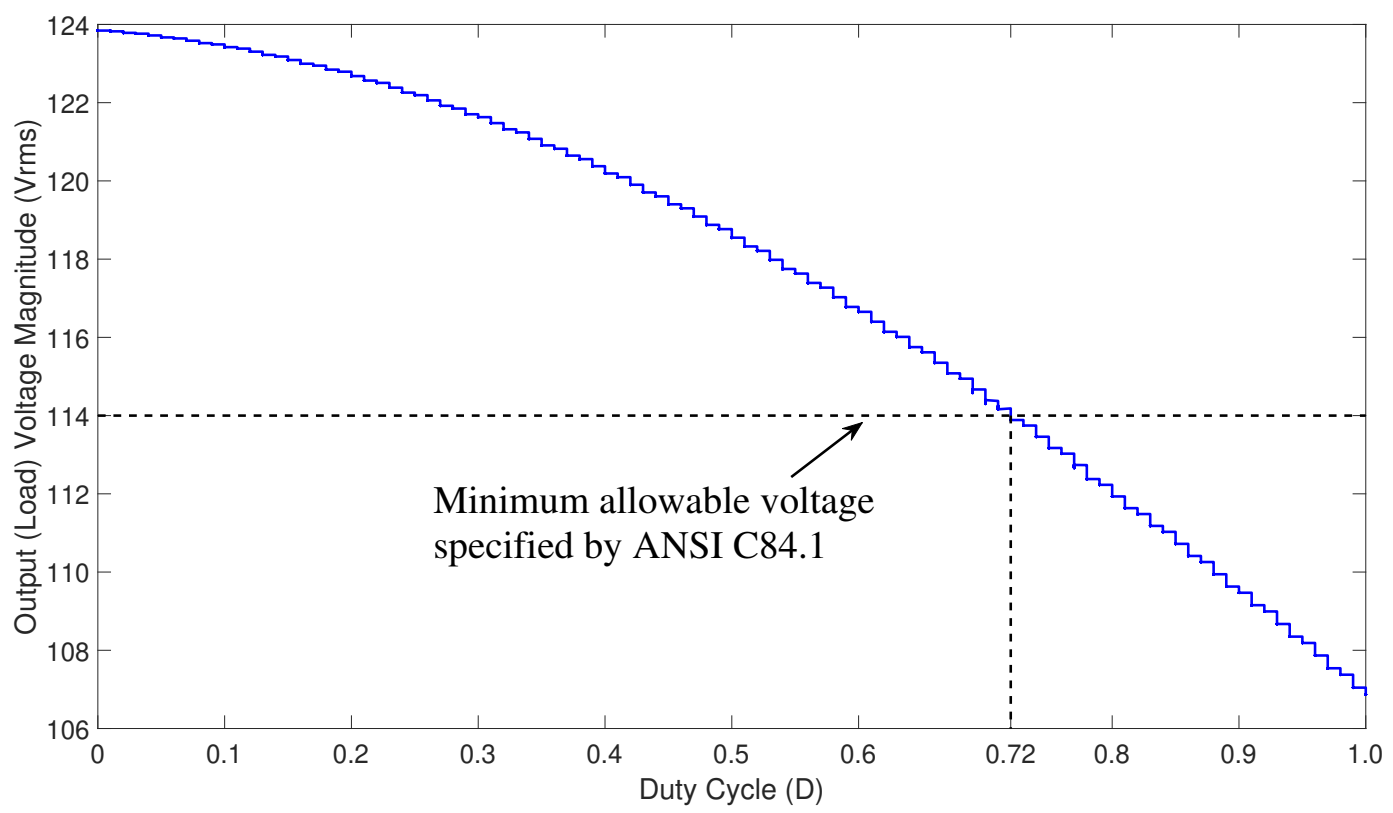

Figure 4.11: Output (Load) voltage variation with duty cycle (D).

$$
\begin{aligned}
Q_{S V C} & =Q_{C}-Q_{L}(D) \\
& =V_{S}^{2}\left(\frac{1}{X_{C}}-\frac{D^{2}}{X_{L}}\right)
\end{aligned}
$$

The simulation was carried out with an ideal input voltage of $120 \mathrm{Vrms}$ at $60 \mathrm{~Hz}$ frequency. A fixed capacitor of $100 \mu \mathrm{F}$ and a switched inductor of $30 \mathrm{mH}$ was used for the simulation. The inductor windings have an internal resistance of $0.25 \Omega$. When the duty cycle is 0 , i.e., the top switch connecting the inductor with the power circuit is open, the reactive power is entirely provided by the fixed capacitor. For a $100 \mu F$ capacitor, the reactive power provided by the capacitor at $120 \mathrm{~V}$ is equal to 543.86 VAR. As the duty cycle increases, the switched inductor compensates the reactive power provided by the fixed capacitor. The net reactive power provided by the RSVC keeps decreasing until the duty cycle reaches 0.66 . The RSVC is operating in "Capacitive Mode" for duty cycles below 0.66. When the duty cycle is equal to 0.66 , the net reactive power supplied by the RSVC is 0 . In order words, the 
reactive power generated by the fixed capacitor is cancelled by the reactive power from the switched inductor. As the duty cycle keeps increasing, the net reactive power provided by the RSVC is negative and the RSVC is said to be operating in "Inductive Mode". When the duty cycle becomes 1 , i.e., the switched inductor is completely on, the net reactive power provided by the RSVC is equal to $-521.68 \mathrm{VAR}^{1}$.

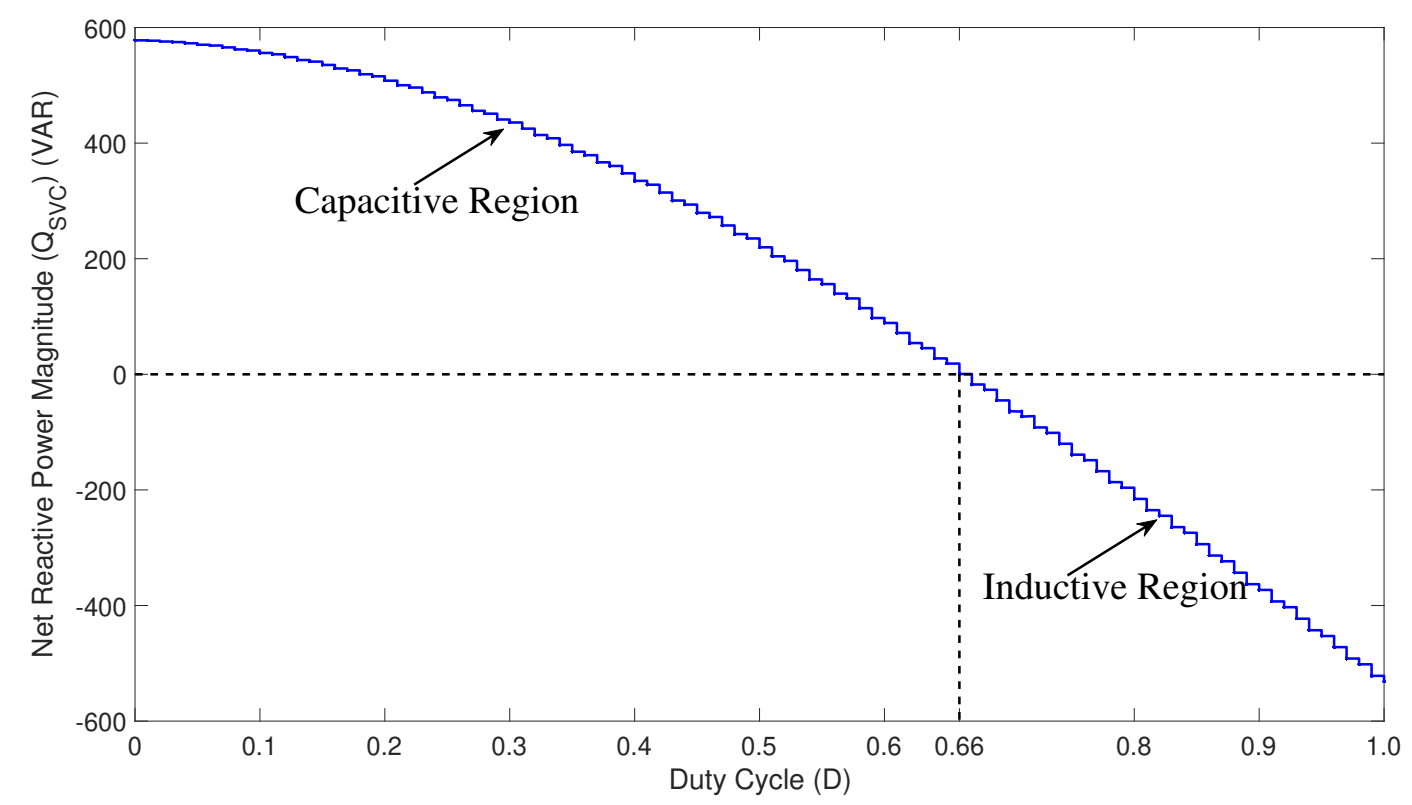

Figure 4.12: Net reactive power $\left(Q_{S V C}\right)$ variation with duty cycle (D).

\subsubsection{Input and Output Power Variation With Duty Cycle}

Figure 4.13 shows the variation of the input and the output power with a change in the duty cycle. The simulation was carried out with an ideal input voltage of $120 \mathrm{Vrms}$ at 60 $\mathrm{Hz}$ frequency. The output voltage is measured across a $2 \mathrm{~kW}$ resistive load. When the duty cycle is 0 , the output power is equal to $2.130 \mathrm{~kW}$, whereas the input power is equal to

\footnotetext{
${ }^{1}$ The reactive power is evaluated at the output load voltage. The output load voltage is equal to approximately equal to $107 \mathrm{~V}$ when the duty cycle is equal to 1 .
} 
$2.138 \mathrm{~kW}$. As the duty cycle increases, the output voltage decreases. For a resistive load, the decrease in output voltage means lower output power. This trend can be seen in Figure 4.13. As the duty cycle increases, the output power goes down. Since the load-demand power decreases with an increase in the duty cycle, the input power at the feeder source bus decreases. For an electric utility, a decrease in power-demand during peak-demand hours can be beneficial in many ways. One such instance is when the electric utility cannot satisfy its demand with its installed capacity. The utilities generally buy energy from another utility in the market therefore incurring additional costs. By integrating RSVC devices in the distribution feeder, utilities can reduce the peak power-demand required and therefore, avoid the cost associated with the purchase of energy from other sources.

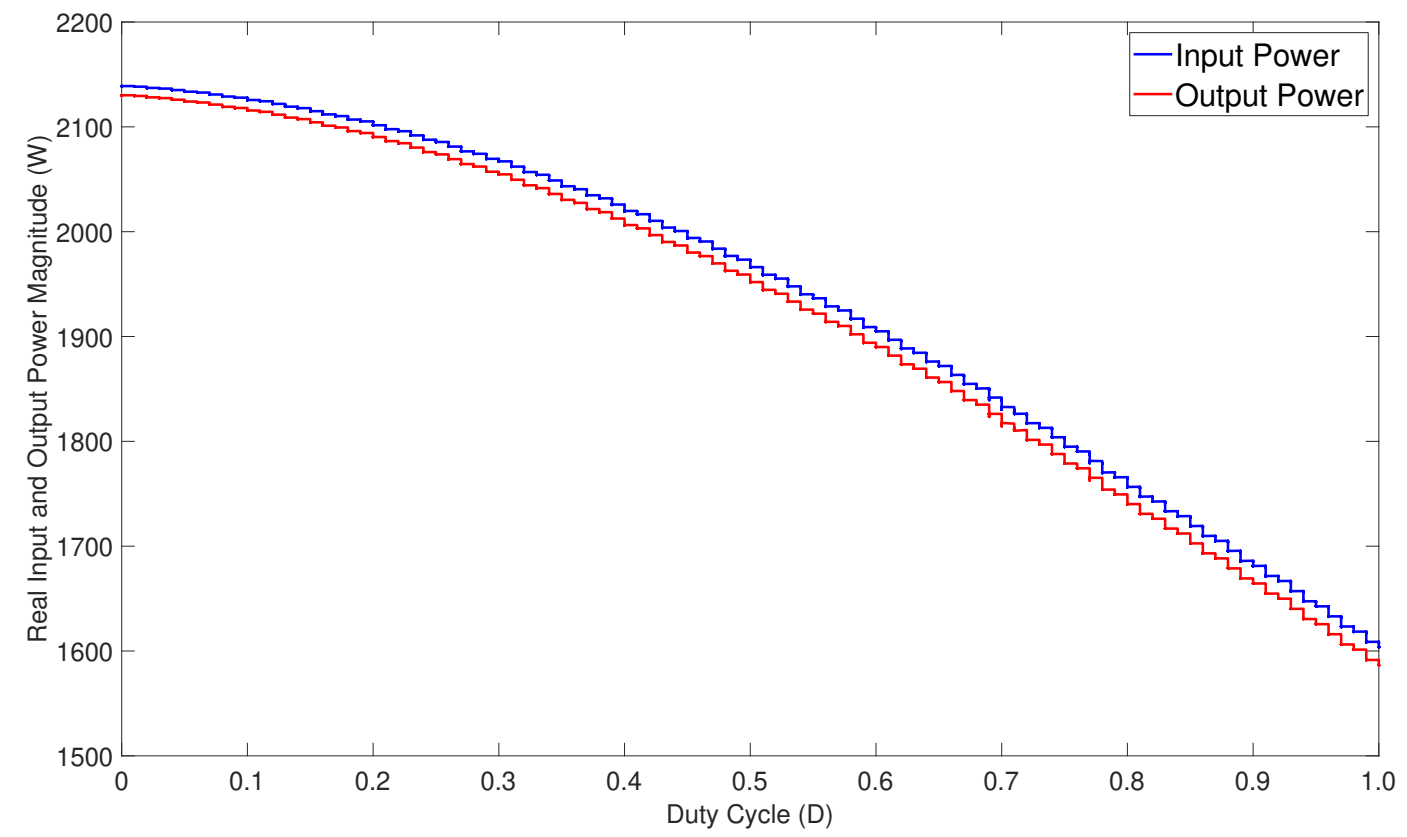

Figure 4.13: Input and output power variation with duty cycle (D). 


\subsection{RSVC Closed-Loop Design}

The previous sections covered the performance of the open-loop RSVC system. As evident from the discussion above, the open-loop RSVC design provides a technological superiority from the previous generations of SVC. However, an open-loop RSVC system does not have the capability to make decisions based on the nature of the input source or the output load. For example, with the variability and unpredictability associated with the renewable generation sources, it becomes difficult to maintain the constant voltage at the loads. The

loads connected with renewable sources undergo the same variability in voltage as it is associated with the source voltage. A feedback control system can enable an open-loop RSVC to maintain a constant voltage at the output by automatically varying the duty cycle. In this way, the intermittent behavior of renewable sources can be neutralized at the loads. Moreover, power companies can adjust reference voltages based on their requirements for different load-demand strategies such as Conversation by Voltage Reduction (CVR).

The following sections will cover in detail the design and performance evaluation for two control loops: (i) an inner, fast-performing voltage regulation control loop, (ii) and an outer, slowly varying power regulation control loop. The purpose of these control loops is to build intelligence to the open-loop RSVC so that it can autonomously perform voltage and power regulation as desired.

\subsubsection{Design of Voltage Regulation Control Loop}

The voltage regulation control loop of the RSVC is constructed to provide the reactive power required to maintain the output voltage at the reference voltage. Figure 4.14 shows the Simulink model of a voltage regulation control loop. The root mean square (RMS) block, as shown in the figure, is used to calculate the magnitude of the output voltage. A 
low-pass filter is used to retain only the DC-component of the signal. The error $(e)$ between the RMS value of the output voltage $V_{\text {out }, \text { rms }}$ and the reference voltage $V_{\text {ref }}$ is fed to the proportional-integral (PI) controller. The output of the PI controller $D^{\prime}(t)$ represents the magnitude of the duty cycle of the bottom bi-directional switch (SW2) used in the RSVC. The signal, $D^{\prime}(t)$ is calculated as follows,

$$
\left|D^{\prime}(t)\right|=\left(K_{p}+\frac{K_{i}}{s}\right)\left(V_{r e f}-V_{o} u t, R M S\right)
$$

where $K_{p}$ and $K_{i}$ are the proportional and integral gains. The output of the PI controller, which generates the duty cycle of the bottom bi-directional switch, is passed through the saturation block. The saturation block is used to limit the output from a minimum value of 0.1 to a maximum value of 0.9 . To generate the duty cycle for the top bi-directional switch, $\left(1-D^{\prime}\right)$ is calculated. This generated duty cycle is provided as input to the state machine (input number 2 as shown in Figure 4.2).

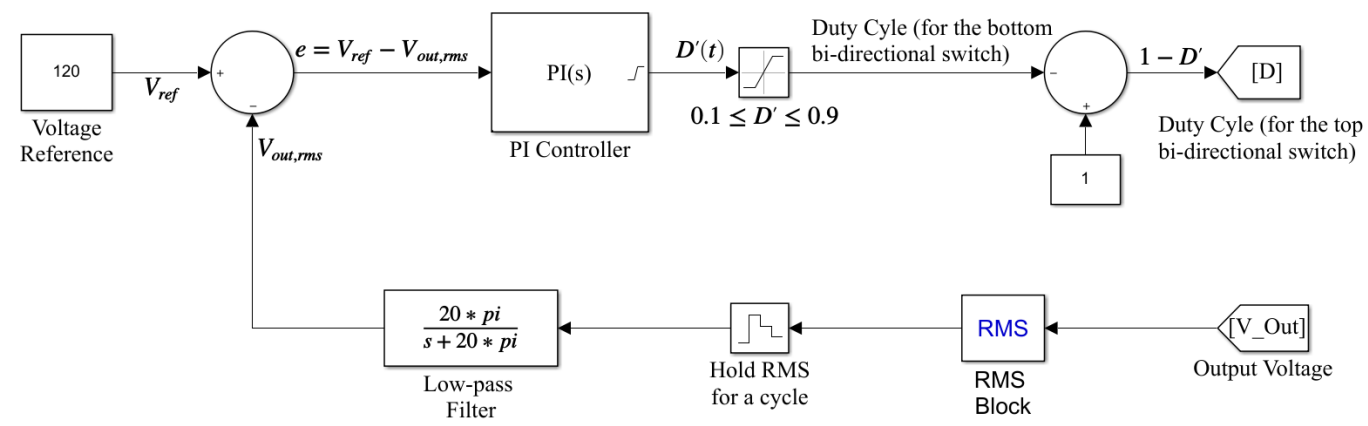

Figure 4.14: Block diagram of the feedback system for the voltage regulation loop of the Residential Static VAR Compensator.

The state machine is responsible for generating the gating signals used in the switching of the bi-directional switches. The PI controller parameters and the saturation block parameters are given in Table 4.1. The parameters $D_{\max }$ in the table shows the maximum 
allowable duty cycle for both bi-directional switches, whereas $D_{\min }$ shows the minimum possible duty cycle. The range of the duty cycle was chosen to provide safe transitioning between steady states and transitional states. In a normal scenario, the duty cycle range can be chosen from $0 \%$ to $100 \%$.

Table 4.1: PI controller and saturation block parameters for the voltage regulation loop.

\begin{tabular}{|c|c|c|c|}
\hline$K_{p}$ & $K_{i}$ & $D_{\max }$ & $D_{\min }$ \\
\hline \hline 0.1 & 1.0 & 0.9 & 0.1 \\
\hline
\end{tabular}

\subsubsection{Integrator Windup in the PI controller}

The control system for a voltage regulation loop presented in Figure 4.14 has a non-linear phenomenon which is related to the limitation for the amount of the reactive power provided by the RSVC. The PI controller produces the duty cycles which can vary from $0 \%$ (reactor is completely disconnected from the regulation bus) to $100 \%$ (reactor is always connected to the regulation bus). When the voltage regulation loop is operating at these extremes, the feedback loop is broken and the control system runs in an open-loop because the output from the PI controller will remain at its limit (saturated), independent of the controller output. Due to this, the integral term will build up (because of the constant error between the reference voltage and the output voltage) and the controller output will become very large. This error build up will prevent the controller from resuming its normal operation once the error decreases and the controller output remains saturated. This non-linear behavior of the controller is termed as integrator windup which leads to large transients and the controller output takes considerable long time before the output is in the range where it doesn't saturate. 
In order to avoid integrator windup, there are two popular integrator anti-windup schemes: (i) clamping method [53] and (ii) back-calculation method [54]. For the clamping method, the integrator in the PI controller is turned on and off depending on the output of the controller. If the output of the controller is in the saturation region, the integrator is switched off otherwise, the integrator keeps integrating the error to produce the controller output to minimize the error. Mathematically, the clamping method can be represented as follows,

$$
K_{i}^{\prime}= \begin{cases}0 & \text { if the controller output is saturated } \\ K_{i} & \text { if the controller output is in linear region }\end{cases}
$$

where $K_{i}^{\prime}$ is the modified integral gain of the PI controller. Figure 4.15 shows the block diagram for the clamping method to avoid the integrator windup.

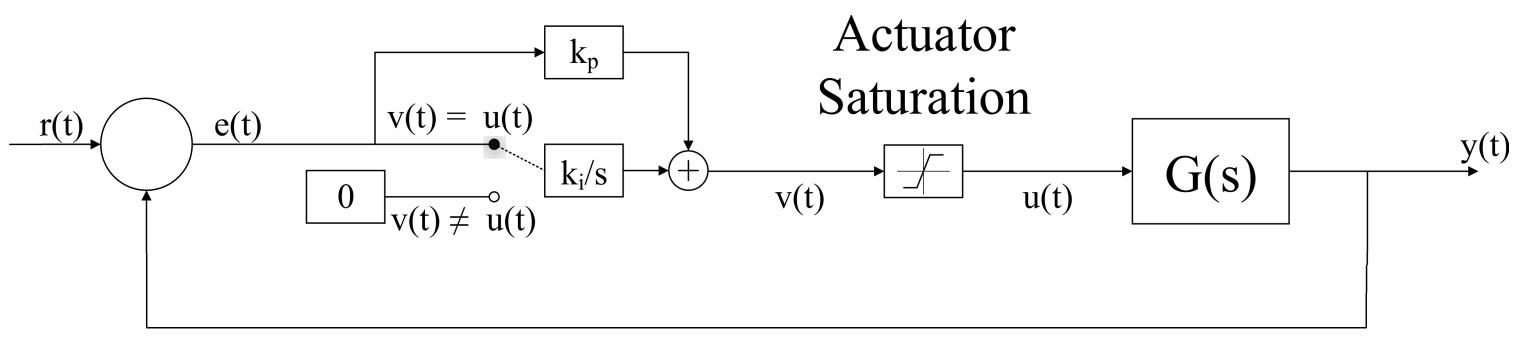

Figure 4.15: Block diagram for clamping method to avoid integrator windup.

In the back-calculation method, the difference between the output of the controller, $u(t)$, and the saturated actuator, $v(t)$, is fed to the input of the integrator through gain $k_{b}$. The difference between the two signals is zero when the controller output is in the linear region. When the controller is in saturation region, the difference is fed back to the integrator such that the difference signal is kept close to 0 . This makes sure that the controller output $u(t)$ remains at (or close to) the saturation limit. Mathematically, this scheme can be represented 
as,

$$
K_{i}^{\prime}=\int K_{i}\left(e+k_{b}(v-u)\right) d t
$$

Normally, the gain $k_{b}$ can not be too large. Otherwise, it will cause the error signal to set to an undesired lower value. The gain $k_{b}$ is generally selected as $1 / K_{p}$ [55] . Figure 4.16 shows the block diagram for the back-calculation method for avoiding the integrator windup. In the RSVC voltage regulation control system, the back-calculation method is used with the gain $k_{b}=1$.

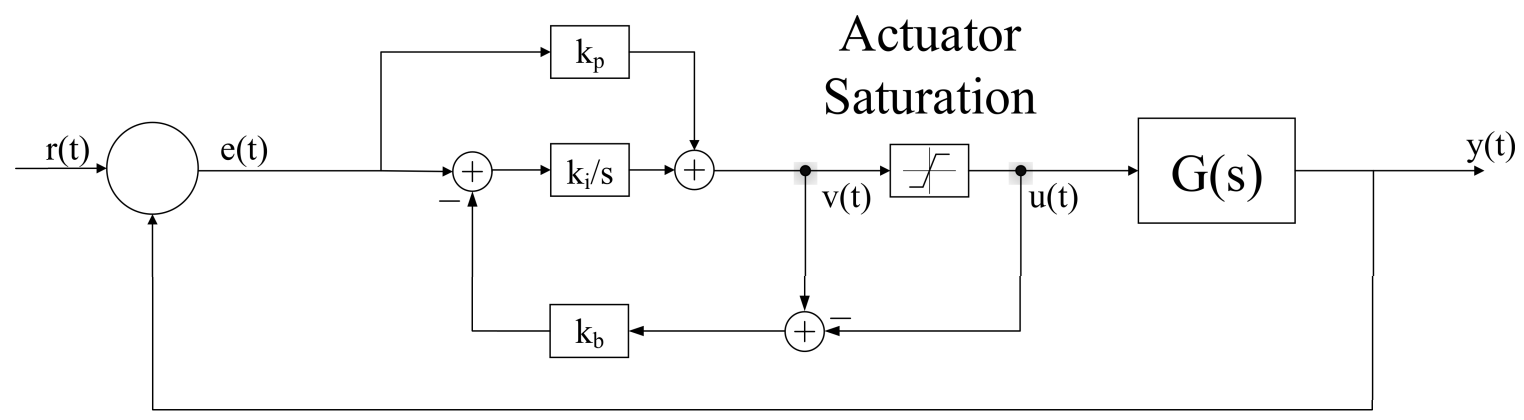

Figure 4.16: Block diagram for back-calculation method to avoid integrator windup.

\subsection{Performance Analysis of Voltage Regulation Control Loop}

\subsubsection{Tracking the Reference Voltage $V_{r e f}$}

The successful operation of the voltage regulation loop depends on its ability to track the reference voltage. To verify the performance of the voltage regulation control loop, a set of simulations were performed. The first simulation was carried out with an ideal input voltage of $120 \mathrm{Vrms}$ at $60 \mathrm{~Hz}$ frequency and the output voltage was measured across a 2 $\mathrm{kW}$ resistive load. Figures 4.17 and 4.18 show the result of the voltage regulation control 
loop. The first plot in Figure 4.17 shows the RMS value for the input voltage, the RMS value for the output voltage, and the reference voltage. For the first half of the simulation, the reference voltage is set to $120 \mathrm{~V}$. The reference voltage is suddenly dropped by $1 \mathrm{~V}$ at 5 sec. At the beginning of the simulation, the output voltage starts at $123.5 \mathrm{~V}$. After the initial system startup errors, the voltage regulation loop successfully tracks the reference voltage. At $5 \mathrm{sec}$, the PI controller experiences a sudden disturbance. The output voltage oscillates but the oscillations last for 4 cycles and the output voltage then tracks the reference voltage at $119 \mathrm{~V}$. The second plot in Figure 4.17 shows the net reactive power during the simulation. When the reference voltage is reduced by $1 \mathrm{~V}$ at $5 \mathrm{sec}$, the net reactive power decreases from 319 VAR to 250 VAR. The decrease in the net reactive power is due to the requirement of more "inductive reactive power" to bring the output voltage to the same level as the reference voltage.
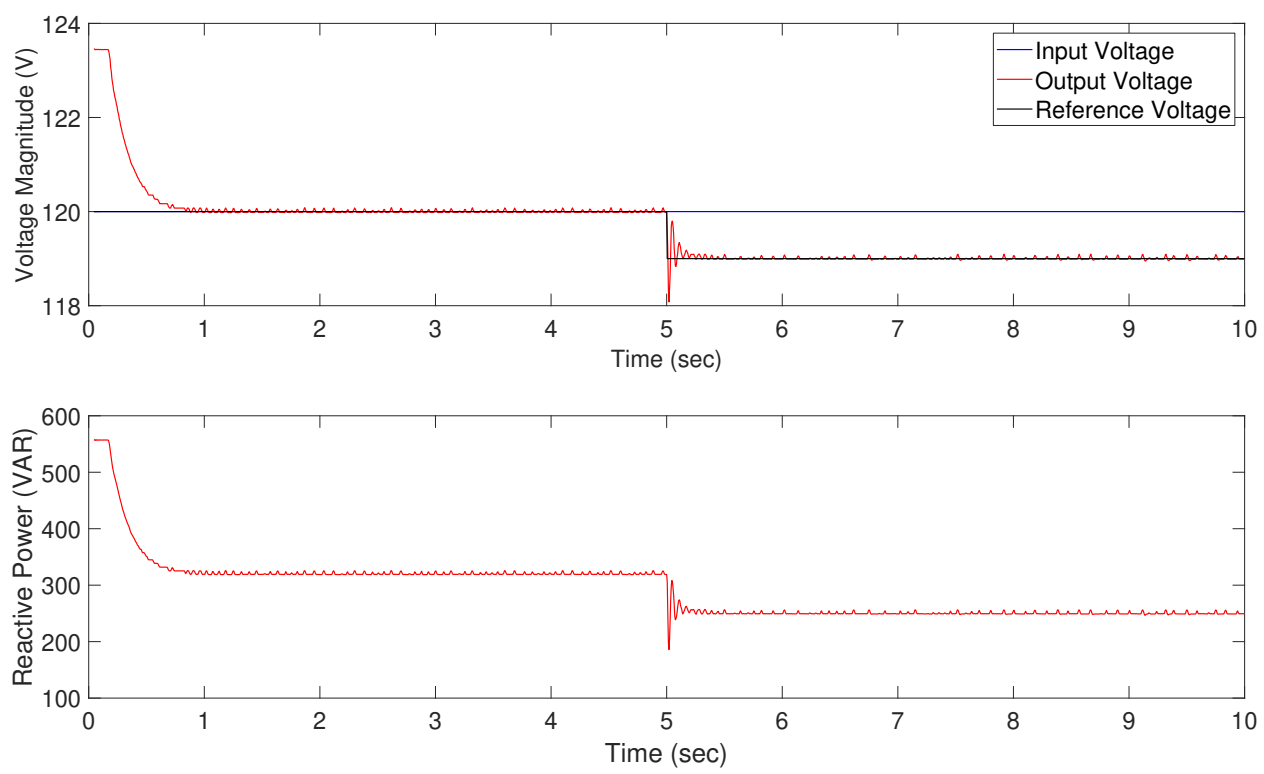

Figure 4.17: Voltages (input, output and reference) and reactive power change caused by the voltage regulation loop operation for an ideal input voltage. 

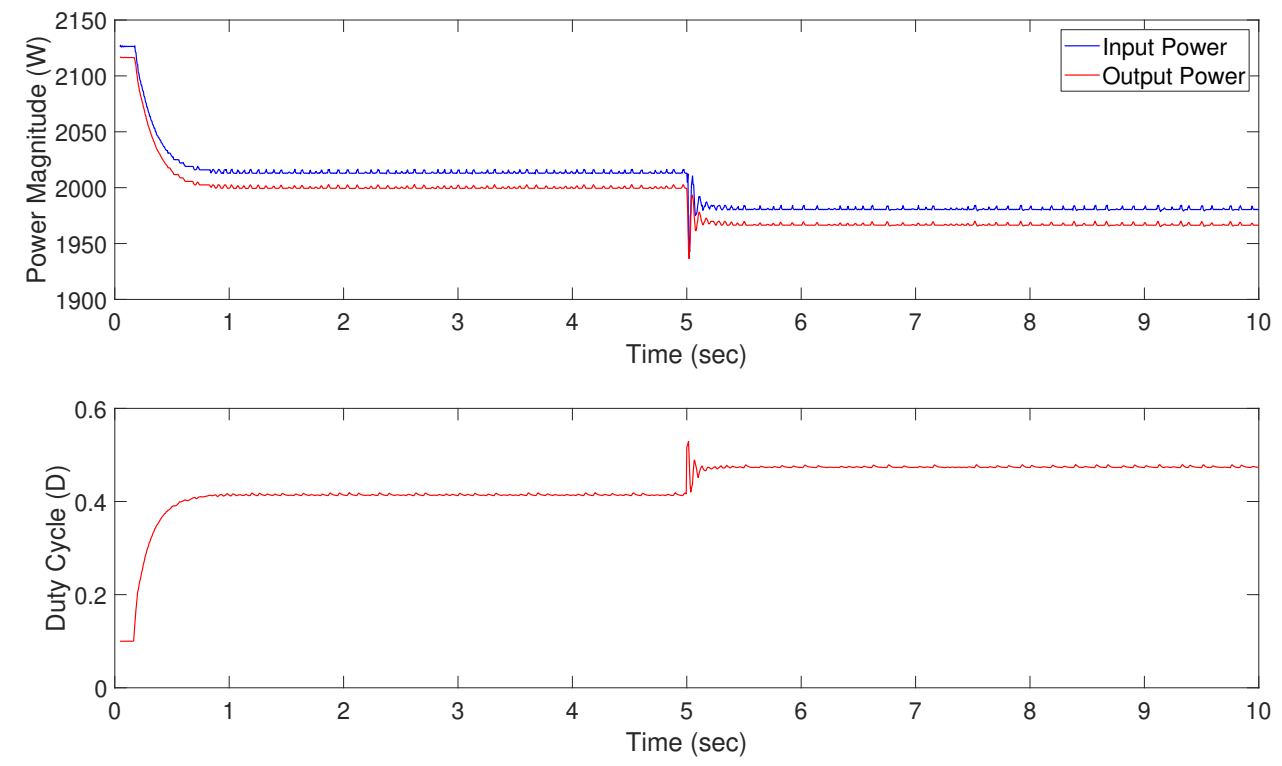

Figure 4.18: Real powers (input and output) and duty cycle change caused by the voltage regulation loop operation for an ideal input voltage.

The first plot in Figure 4.18 shows the input and output load power. The input power decrease from $2.01 \mathrm{~kW}$ at $120 \mathrm{~V}$ to $1.98 \mathrm{~kW}$ at $119 \mathrm{~V}$. Similarly, the output power decreases from $2.0 \mathrm{~kW}$ to $1.97 \mathrm{~kW}$. The decrease in the input and output power is due to decrease in the load voltage from $120 \mathrm{~V}$ to $119 \mathrm{~V}$. The second plot in Figure 4.18 shows the change in the duty cycle when the reference voltage drops from $120 \mathrm{~V}$ to $119 \mathrm{~V}$. The duty cycle increases from 0.41 to 0.47 after the change in reference voltage.

\subsubsection{Voltage Regulation Loop Performance With Variable Input Voltage}

Figures 4.19 and 4.20 represent the performance of the voltage regulation loop with variable (noisy) input voltage. The variable input voltage is the replica of the voltage generated due to solar irradiance at a PV plant as shown in Figure 1.3. The data in Figure 1.3 was obtained at 1 second intervals. However, for faster simulation, the timestamp of the data was changed to $2.4 \mathrm{msec}$. Therefore, the entire data (originally for 7 hours from $6 \mathrm{AM}$ 
till 1 PM) was fitted to 1 minute. The first plot in Figure 4.19 shows the input and output voltage for the pre-recorded solar irradiance voltage profile. During this interval, the RSVC is not supplying any reactive power. It can be seen that the output voltage follows the input voltage profile. After 60 seconds, the input voltage is held at a constant voltage of $120 \mathrm{~V}$ for 15 seconds. After this time, the same solar-irradiance voltage profile is reapplied at the input. However, during this interval, RSVC is allowed to supply the reactive power to maintain the load voltage at the reference voltage of $120 \mathrm{~V}$. It can be seen that the voltage regulation control loop responds to the changes in the input voltage and provides the appropriate reactive power to maintain the output voltage at $120 \mathrm{~V}$. The second plot in Figure 4.19 shows the net reactive power provided by the RSVC.
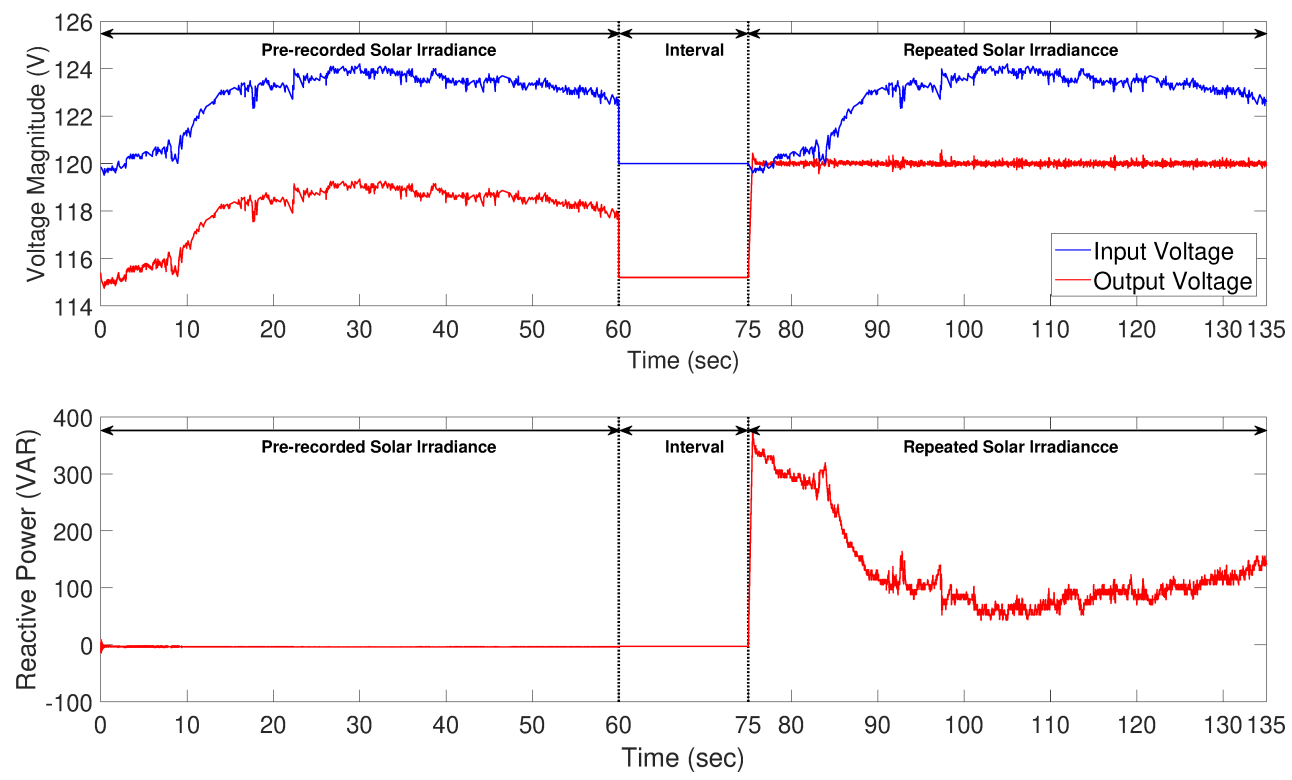

Figure 4.19: Voltages (input, output and reference) and reactive power change caused by the voltage regulation loop operation for a variable input voltage.

The first plot in Figure 4.20 shows the input and output power for the system with and without the RSVC. When the RSVC is turned off, i.e., it is not contributing any reactive power to the power system, the output power fluctuates as the input voltage changes. How- 

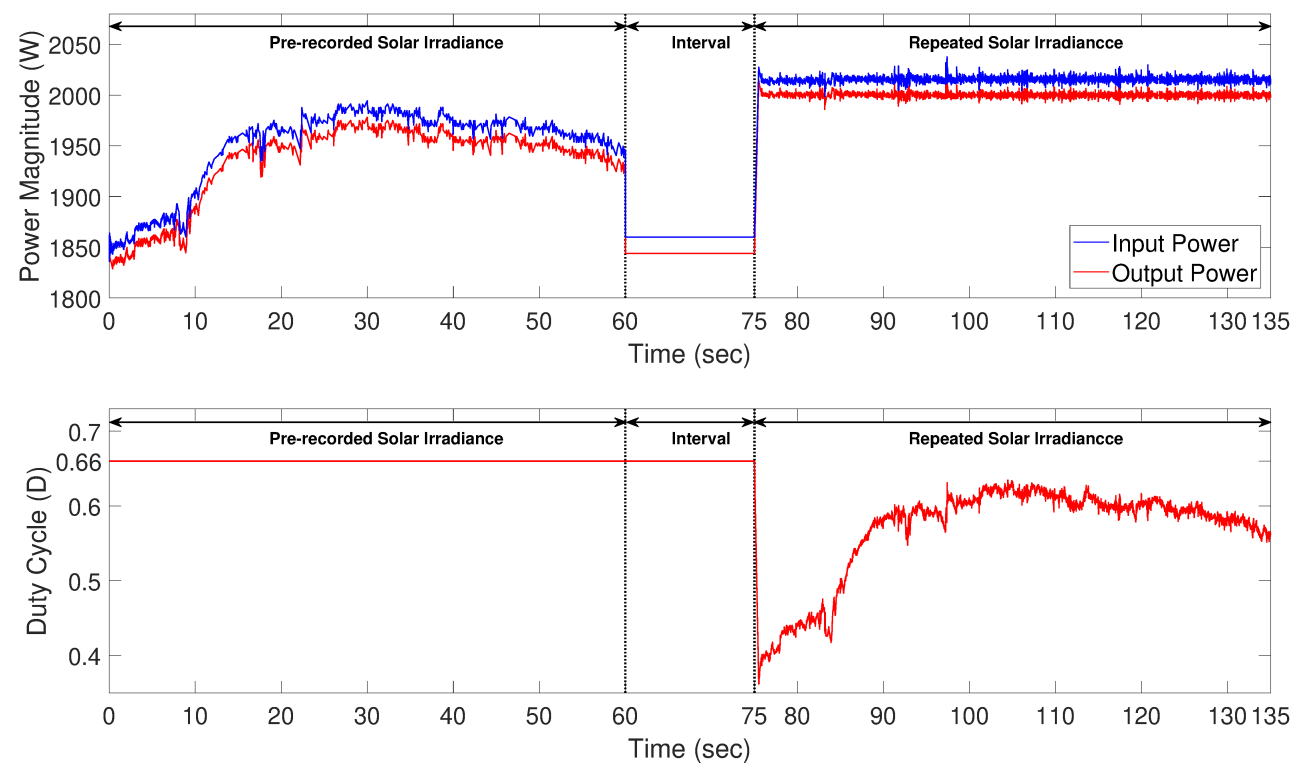

Figure 4.20: Real powers (input and output) and duty cycle change caused by the voltage regulation loop operation for a variable input voltage.

ever, when the RSVC voltage regulation loop is allowed to operate, the input and output power stay constant despite the variability of the input voltage. This result holds importance for electric utilities for planning the load power-demand when the majority of the input supply is generated via renewable resources. The second plot in Figure 4.20 shows the variation in the duty cycle during the simulation. It should be noted that during the initial part of the simulation, the duty cycle was held at 0.66. At this particular duty cycle, the switched reactor inductive reactive power completely cancels the fixed capacitor capacitive reactive power. Hence, the net reactive power injected by the RSVC is approximately equal to zero. Whereas as in the second half of the simulation, the duty cycle changes such that the output voltage remains at $120 \mathrm{~V}$. 


\subsubsection{Load Power Demand Shaving in a Distribution Feeder}

Figures 4.21 and 4.22 show the result of load power-demand shaving during peak-demand hours. The RSVC has the ability to perform peak shaving at the distribution transformer or at the customer level. Traditional peak-demand shaving or CVR requires the voltage to be reduced across the feeder. While such approaches are still possible, the RSVC allows performing peak-shaving for a specific distribution transformer or a particular customer(s) and can enable electric utilities to deploy better strategies for peak-shaving using CVR. Chapter 6 presents the results for the study performed for RSVC-enhanced CVR.

The first plot in Figure 4.21 shows the input and output voltage of the power system with and without the RSVC. When the RSVC is not connected to the power system, i.e., the net reactive power supplied by the RSVC is 0 , the output voltage follows the trend of the variable input voltage. However, when the same input voltage profile is re-applied with the RSVC connected to the regulation bus, the output voltage tracks the reference voltage, which is set at $114 \mathrm{~V}$. If the loads connected to the power system have a positive real CVR factor, i.e., the real power decreases with the decrease in the voltage, then the electrical utilities can see benefits from the power saved. The second plot in Figure 4.21 shows the amount of net reactive power supplied by the RSVC. When the RSVC is turned on, the net reactive power provided by the RSVC is negative. Since the output voltage is tracking the reference voltage at $114 \mathrm{~V}$, the RSVC has to operate in the inductive region to reduce the output voltage.

The first plot in Figure 4.22 shows the input and output power of the power system with and without the RSVC. It can be seen that the output power decreases when the output voltage drops to $114 \mathrm{~V}$. The output power decreases from $2.0 \mathrm{~kW}$ at $120 \mathrm{~V}$ to $1.8 \mathrm{~kW}$ at $114 \mathrm{~V}$. The second plot of the Figure 4.22 shows the variation of the duty cycle to maintain 
the output voltage at the reference voltage of $114 \mathrm{~V}$.
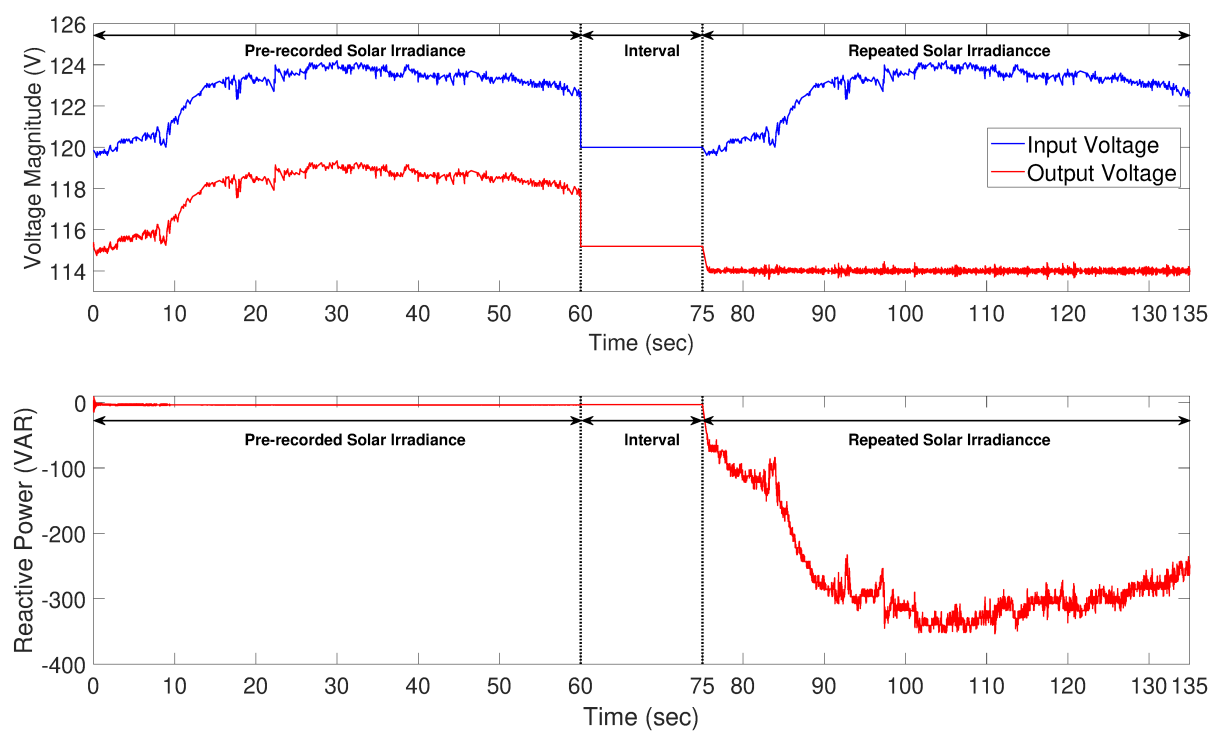

Figure 4.21: Voltages (input, output and reference) and reactive power change caused by voltage regulation loop operation for a variable input voltage to achieve peak-demand shaving.
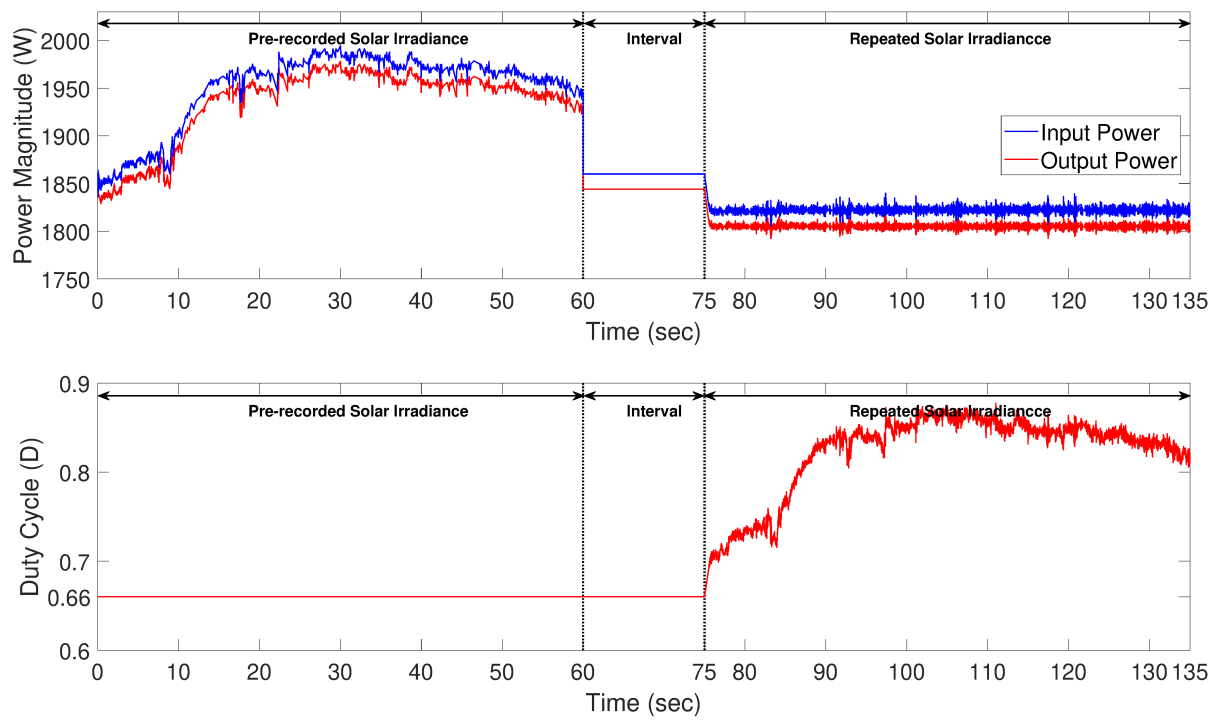

Figure 4.22: Real powers (input and output) and duty cycle change caused by voltage regulation loop operation for a variable input voltage to achieve peak-demand shaving. 


\subsection{Design of Power Regulation Control Loop}

The objective of this task is to design and implement the outer power regulation loop for the RSVC to achieve Minimum Power (or optimal power) Point Tracking (MinPPT). This task consisted of designing a minimum power point algorithm that will track the power consumed by a certain distribution transformer or residential customers to reduce the customer power to the minimum possible while maintaining the customer service voltage within the permissible Range A in ANSI C84.1 Standard. The outer power regulation loop is designed in Simulink with the RSVC model and the inner voltage regulation loop as explained in the previous sections. The power regulation is designed as a slower loop than the voltage regulation loop to build an inherent time decoupling between the two loops and to avoid a possible control system interaction.

The algorithm for the MinPPT is developed by using (inverting) the logic of a common Maximum Power Point Tracking (MPPT) technique and the Perturbation and Observation (P\&O) method [56-64]. By applying a MinPPT and the P\&O method, the RSVC can maintain its operating point around the minimum power point, which means a lower customer power consumption. In this approach, a perturbation $( \pm \Delta V)$ is periodically introduced to the RSVC system, and then the observation (output power) is compared with the previous value. If the power decreases due to the perturbation, then the perturbation continues in the same direction during the next perturbation cycle. Otherwise, the perturbation voltage moves toward the opposite direction. In this way, the operating point of the RSVC system gradually moves toward the minimum power point and stabilizes around the minimum

power point in the steady state. The size of the perturbation step determines the speed of reaching the minimum power point and the size of the oscillations. A large perturbation step yields a fast tracking speed but results in large oscillations around the minimum power 
point. Therefore, the perturbation step should be adjusted by making a trade-off between a fast-speed response and lower magnitude of oscillations at steady state.

Figure 4.23 shows the complete Simulink block diagram implementation for the RSVC power regulation loop. A power measurement block is used for measuring the active real power $(\mathrm{P})$ of the output resistive load. The power measurement block outputs the power (real and reactive) for the fundamental frequency component. For the RSVC power regulation loop, the output power and output voltage are used as an input to the power regulation loop. The mean block is used to calculate a mean of 60 cycles. The mean values of the output voltage and the output power are used as input to the Power Loop P/O Decision subsystem. The subsystem operates at a desired speed for operating the power regulation loop. For the simulation, the power regulation loop updates at an interval of every 2 seconds. The contents of the subsystem are shown in Figure 4.24.

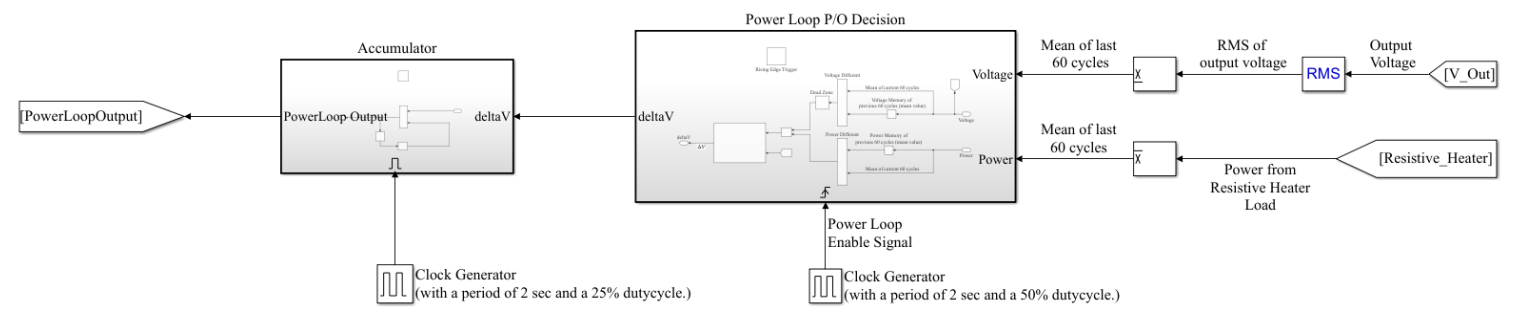

Figure 4.23: Complete Simulink block diagram of power regulation loop.

Memory blocks are used for storing the previous output voltage and the output power for the duration of the next rising clock edge $(2 \mathrm{sec})$, which represents the period of one perturbation cycle. The voltage difference $(\Delta V)$ and power difference $(\Delta P)$ are computed, which determines the next perturbation direction. At this point, $\Delta V$ (and $\Delta P$ ) can be computed by subtracting the previous perturbation cycle output voltage (and power) from the current perturbation cycle output voltage (and power). If $\Delta V$ and $\Delta P$ are both positive, it means that the RSVC operation point is moving away from the minimum power point 


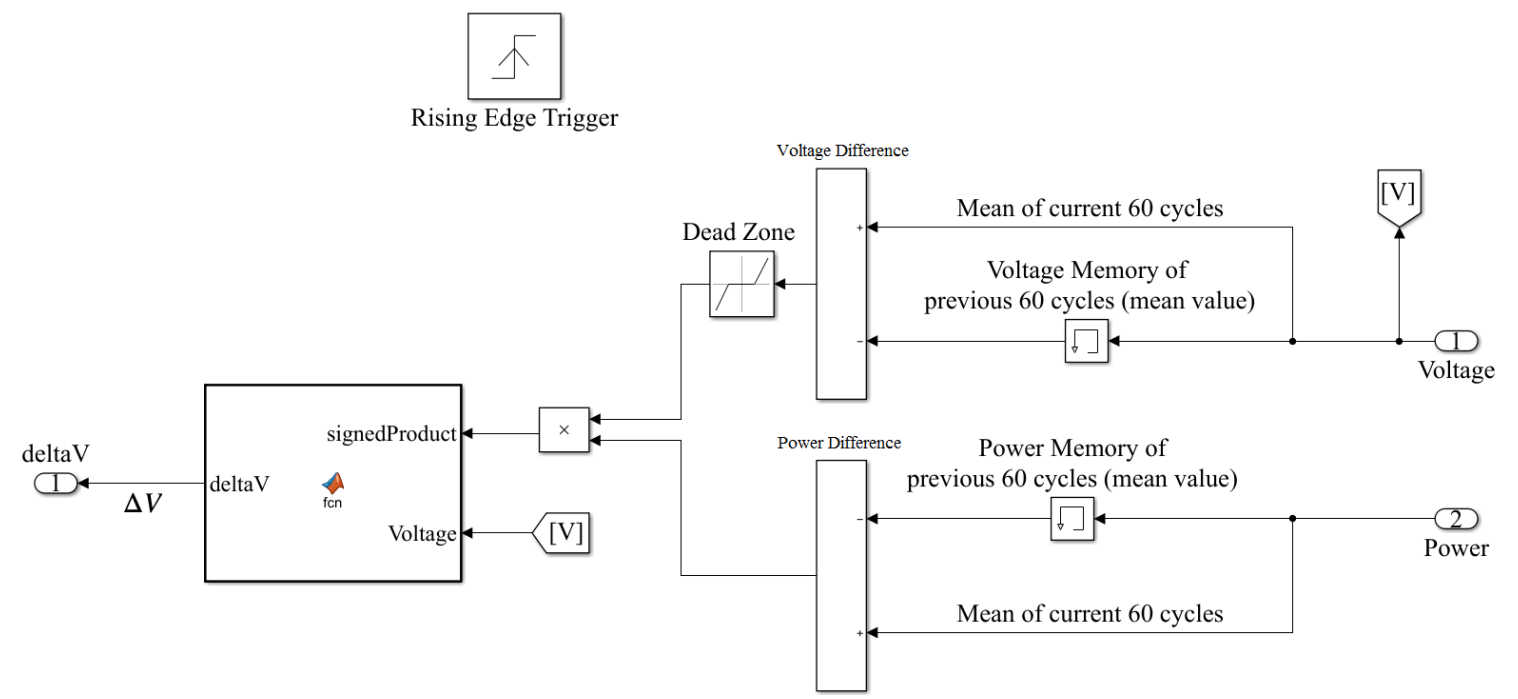

Figure 4.24: Block diagram of Power Loop P/O Decision subsystem.

because of the positive perturbation. Therefore, the next perturbation goes in the opposite (negative) direction.

The output of the power regulation loop is summarized as follows:

- $\Delta V>0, \Delta P>0$, decrease the reference voltage.

- $\Delta V>0, \Delta P<0$, increase the reference voltage.

- $\Delta V<0, \Delta P>0$, increase the reference voltage.

- $\Delta V<0, \Delta P<0$, decrease the reference voltage.

This sequence can be simplified by taking a product of $(\Delta V)$ and $(\Delta P)$. If the signed product is positive, the reference voltage decreases, whereas if the signed product is negative, the reference voltage increases. Pseudocode for the Matlab function to decide the direction of the perturbation is given in Algorithm 1.

The output of the function block specifies the direction of the perturbation. To avoid a step-change in the reference voltage, the reference voltage is increased or decreased 


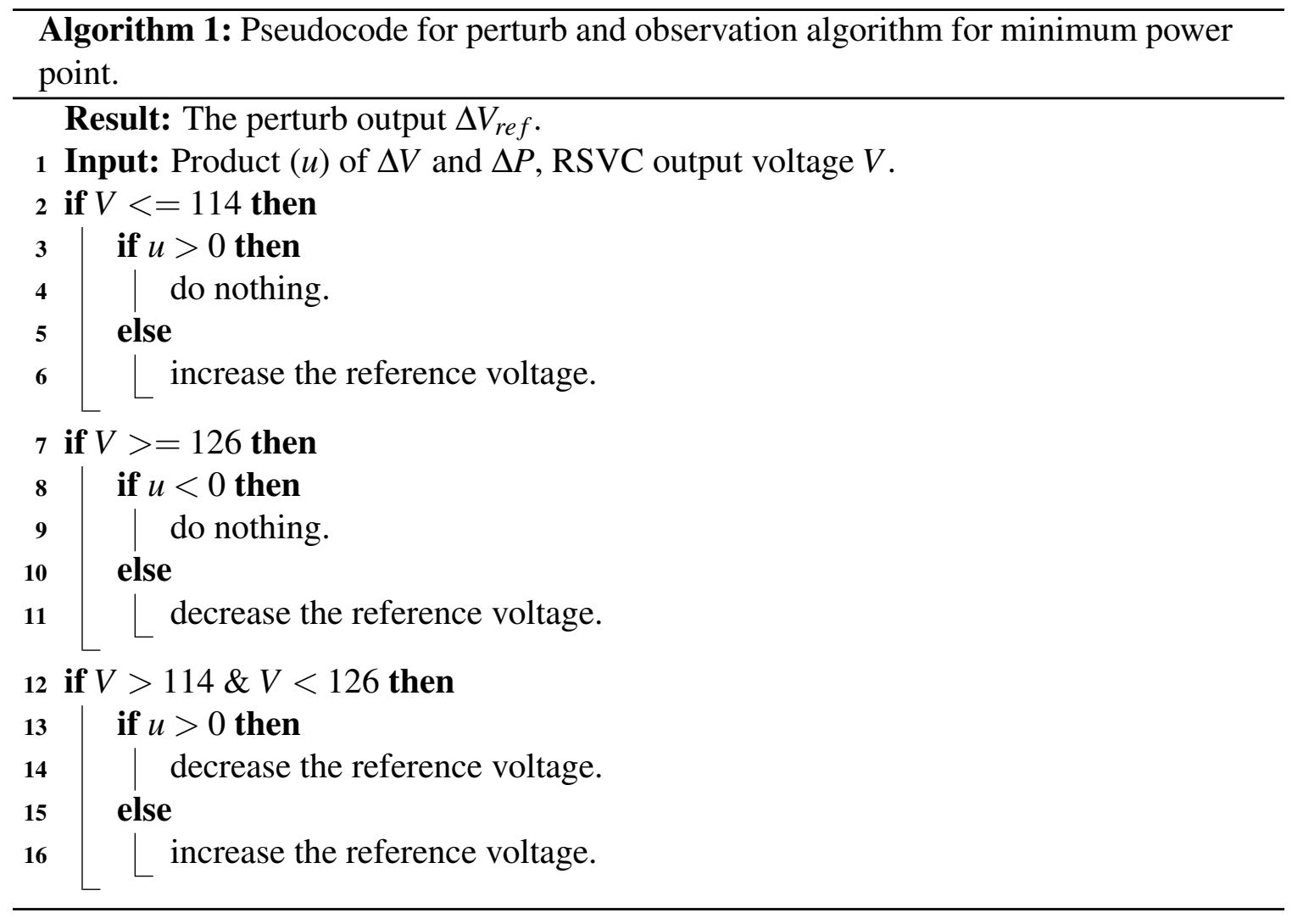

linearly. For the simulation, a change of $\pm 0.5 \mathrm{~V}$ in the reference voltage was chosen. This change was achieved in 30 cycles (or 0.5 seconds) with a $0.05 \mathrm{~V}$ change in the reference voltage every 3 cycles of the source frequency $(60 \mathrm{~Hz})$. The Figure 4.25 shows the block diagram of the Accumulator subsystem which is used to achieve the change in reference voltage linearly.

After the next perturbation is introduced to the RSVC, the voltage regulation loop reacts to the perturbation and brings the RSVC system to the new reference voltage level. Once the RSVC voltage is stable, the power regulation loop determines the next perturbation by checking the sign of voltage difference $(\Delta V)$ and the power difference $(\Delta P)$. By repeating all of these steps, the RSVC operation point eventually moves to the minimum power point and oscillates around that point. 


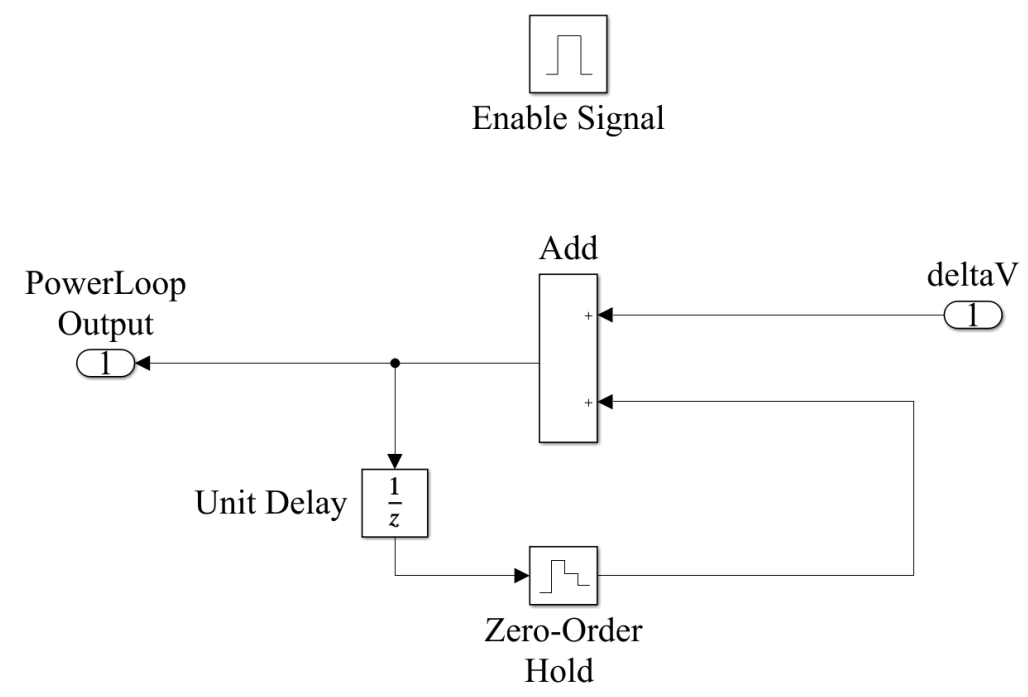

Figure 4.25: Block diagram of Accumulator subsystem.

\subsection{Performance Analysis of Power Regulation Control Loop}

\subsubsection{Tracking the Power for a $2 \mathrm{~kW}$ Resistive Load}

Figures 4.26 and 4.27 show the simulation results for the RSVC outer power regulation loop. To verify the performance of the power regulation control loop, a set of simulations were performed. The first simulation was carried out with an ideal input voltage of 120 Vrms at $60 \mathrm{~Hz}$ frequency and the output voltage and power were measured across a 2 $\mathrm{kW}$ resistive load. The first plot in Figure 4.26 shows the RSVC input voltage, the output voltage power, and the reference voltage. The power regulation loop triggers at the rising edge of a clock which is working at a 2 second time period. At the start of the simulation, the reference voltage is set to $120 \mathrm{~V}$. At the 2-second mark, the power regulation loop calculates the first perturbation step. The output of the power regulation loop decides the direction in which the reference voltage can change. There are three possible outcomes of power regulation: (i) increase the reference voltage, (ii) decrease the reference voltage, 
(iii) no change in the reference voltage.

During the first perturbation, the power regulation loop outputs to decrease the reference voltage by $0.5 \mathrm{~V}$. The decrease in the reference voltage is carried out in 10 steps during a 30 cycles ( $0.5 \mathrm{sec}$ with a $0.05 \mathrm{~V}$ change in the reference voltage every 3 cycles) interval. The negative ramp for the decrease in reference voltage is shown by the vertical lines in Figure 4.26. As expected, the reference voltage decreases from $120 \mathrm{~V}$ to $114 \mathrm{~V}$ as a result of the output from the power regulation loop.

The second plot in Figure 4.26 shows the input and output power across the $2 \mathrm{~kW}$ resistive load. The output power decreases from $2 \mathrm{~kW}$ to $1.8 \mathrm{~kW}$ by lowering the voltage from $120 \mathrm{~V}$ to $114 \mathrm{~V}$. The power regulation loop keeps monitoring the output voltage and output power at every 2 second interval. However, a further decrease in the voltage is prohibited by the ANSI C84.1 standard. If the composition of the load changes when the minimum power point loop is operational, the control loop should respond to the nature of the load and thus adjust the reference voltage so minimum power is consumed by the loads.

The first plot in Figure 4.27 shows the net reactive power supplied by RSVC to maintain the output voltage at the reference voltage. As the output voltage decreases, the RSVC becomes increasingly inductive. Therefore, the net reactive power supplied by the RSVC decreases. The second plot in Figure 4.27 shows the change in the duty cycle during the simulation. The duty cycle increases to provide more inductive reactive power, which is consistent with the developed theory.

\subsubsection{Tracking the Power for a Resistive Heater Load}

Figure 4.28 and 4.29 show the simulation result of the RSVC power regulation loop for a resistive heater. The resistive heater was simulated in Simulink as a Matlab function by using the ZIP coefficients given in [34]. The outputs of the Matlab function are the real and 

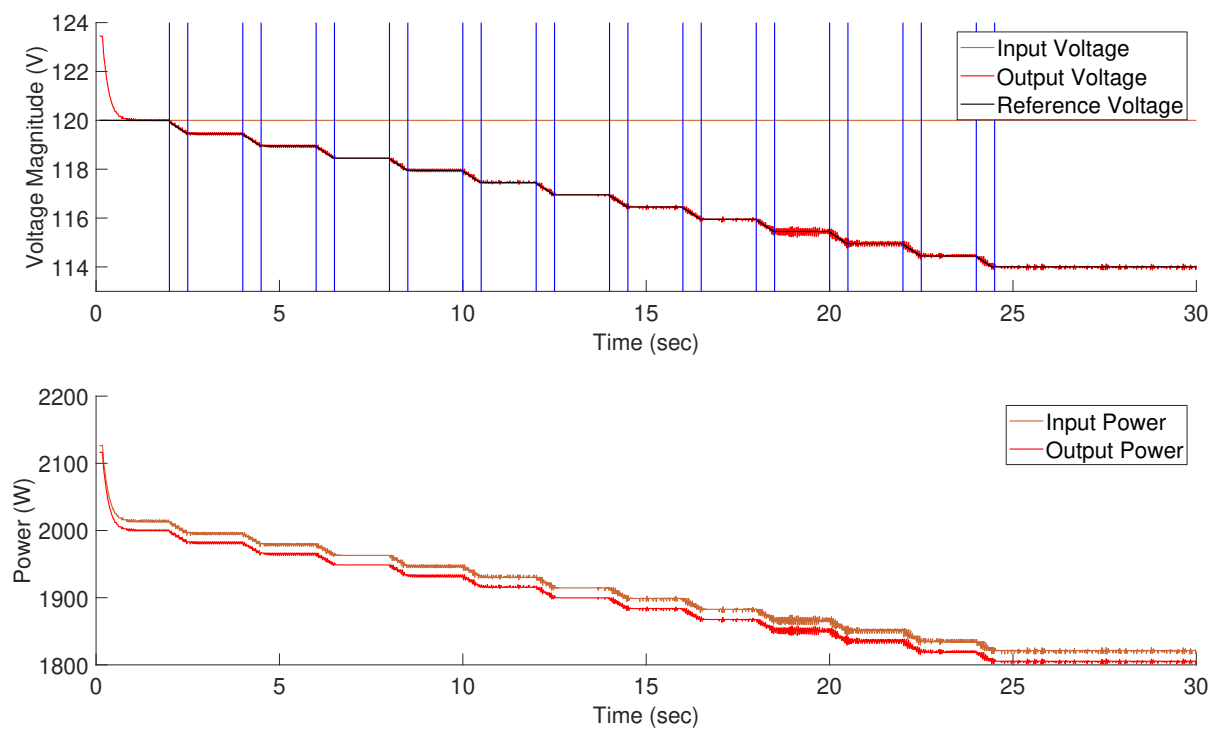

Figure 4.26: Voltages (input, output and reference) and real power (input and output) change caused by power regulation loop operation for an ideal input voltage with a $2 \mathrm{~kW}$ purely resistive load.
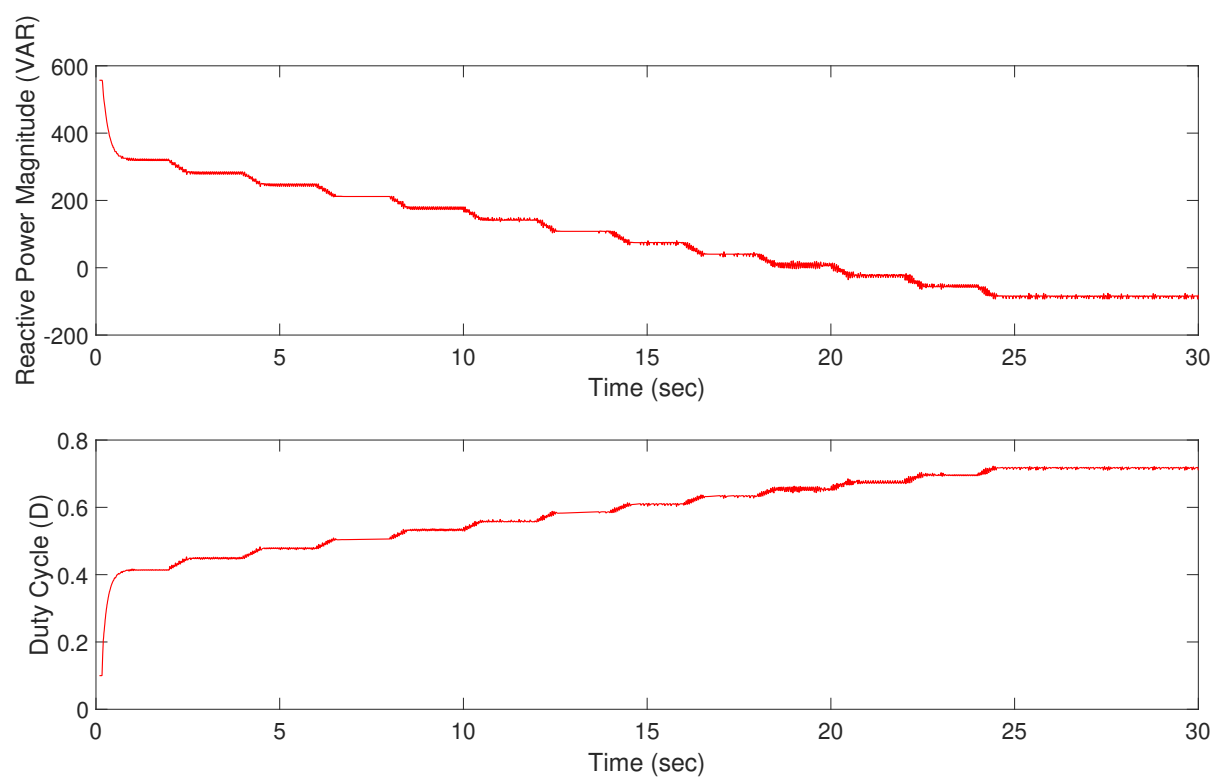

Figure 4.27: Net reactive power and duty cycle change caused by power regulation loop operation for an ideal input voltage with a $2 \mathrm{~kW}$ purely resistive load. 
reactive power of the resistive heater. The inputs to the RSVC power regulation loop were the resistive heater voltage and power. The first plot in Figure 4.28 shows the input, output, and reference voltage. It can be seen that the reference voltage changes from $120 \mathrm{~V}$ to 114 $\mathrm{V}$ to reduce the power consumed by the resistive heater. The change in the power of the resistive heater is from $915 \mathrm{~W}$ to $830 \mathrm{~W}$. Similarly, the net reactive power provided by the RSVC decreases, whereas the duty cycle increases, as shown in Figure 4.29.
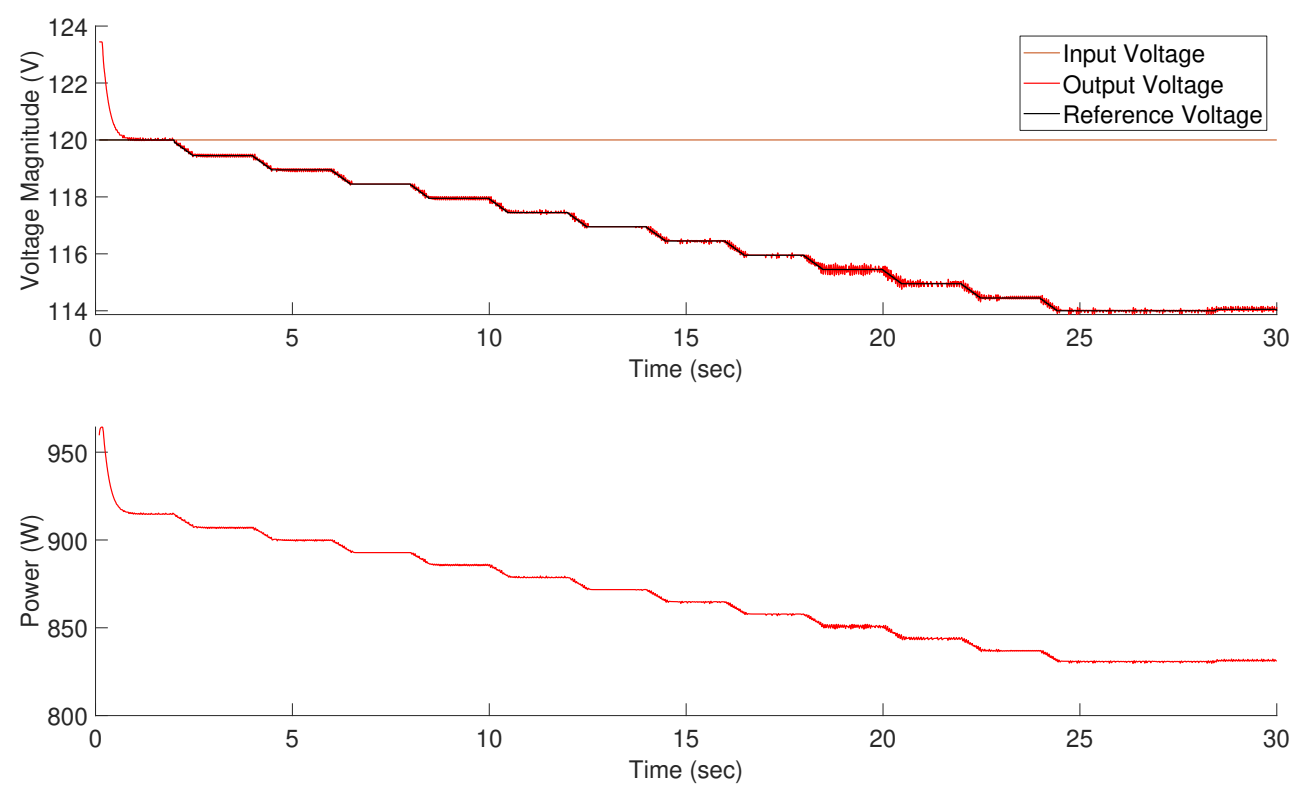

Figure 4.28: Voltages (input, output and reference) and real power (output) change caused by power regulation loop operation for an ideal input voltage with a resistive heater load.

\subsubsection{Tracking the Power for a Resistive Heater Load with Variable Input Voltage}

Figures 4.30 and 4.31 show the simulation results of the RSVC power regulation loop for a resistive heater. The first plot in Figure 4.30 shows the input, output, and reference voltage. The reference voltage changes from $120 \mathrm{~V}$ to $114 \mathrm{~V}$ to reduce the power consumed by the resistive heater even in the presences of the variable/noisy input voltage. The change in the 

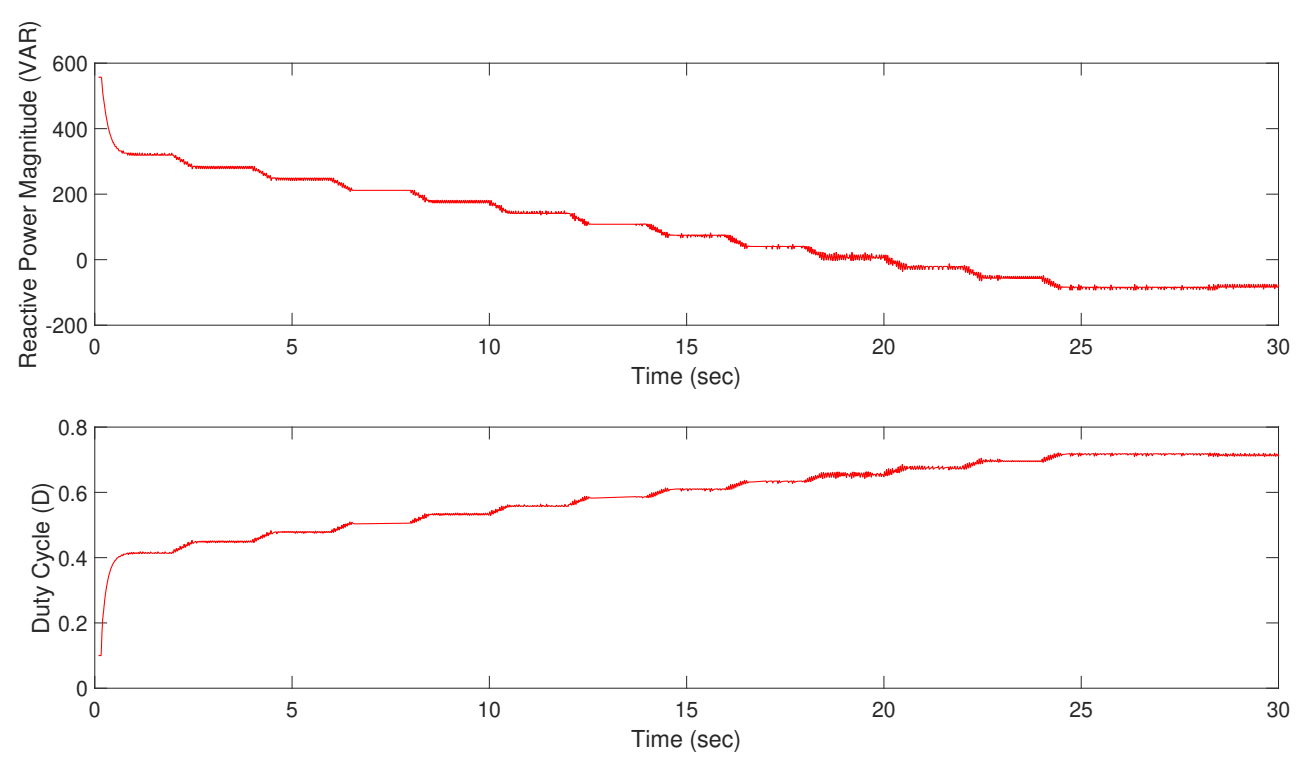

Figure 4.29: Net reactive power and duty cycle change caused by power regulation loop operation for an ideal input voltage with a resistive heater load.

power of the resistive heater is similar to the previous simulation i.e., it changes from 915 $\mathrm{W}$ to $830 \mathrm{~W}$.

Similarly, the net reactive power provided by the RSVC decreases, whereas the duty cycle increases as shown in Figure 4.29. The simulation result shows that both control loops are efficiently regulating the voltage and power despite of the input voltage fluctuations. This result is specifically useful when the optimal power-demand is required in case of limited renewable resources.

\subsubsection{Tracking the Power for Loads with Negative CVR Factor}

The previous section presented the performance of the power regulation loop with loads that have positive CVR factors. Normally, when the voltage decreases, the real power of the loads also decreases; when the voltage increases the real power increases, hence the positive CVR factor. However, this is not generally true. In some cases, the decrease in voltage 

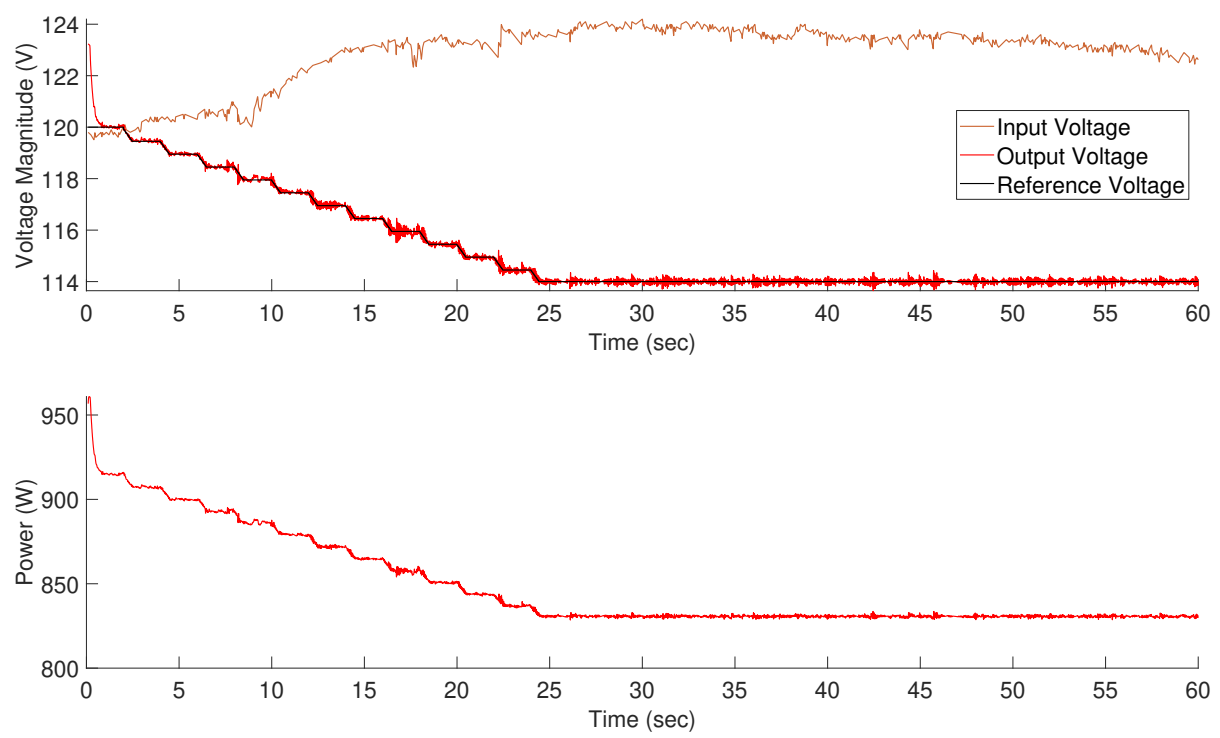

Figure 4.30: Voltages (input, output and reference) and real power (output) change caused by power regulation loop operation for a variable input voltage with a resistive heater load.
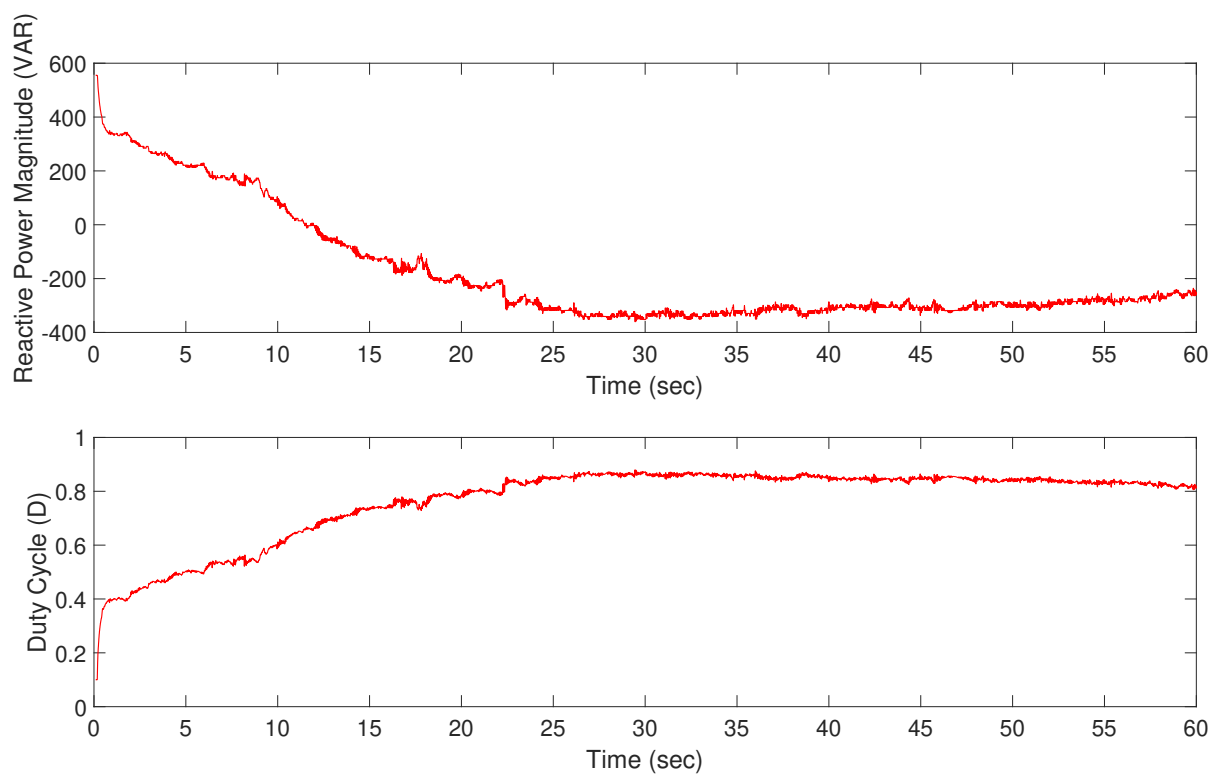

Figure 4.31: Net reactive power and duty cycle change caused by power regulation loop operation for a variable input voltage with a resistive heater load. 
results in more power drawn by the loads. In those cases, the real CVR factor $\left(C V R_{P}\right)$ is negative. For such loads, decreasing the voltage have negative consequences while implementing CVR. The power regulation control system, presented in this dissertation, monitors the power utilization by the loads having negative CVR factors and, therefore, works to minimize the power utilized by such loads by increasing the reference voltage.

Figure 4.32 represents an example of the $\operatorname{load}^{2}$ depicting a negative CVR factor. It can be seen that the power consumption of the load increases as the voltage is reduced. Therefore, to minimize the power drawn by such a load, the terminal voltage at the load should be increased.

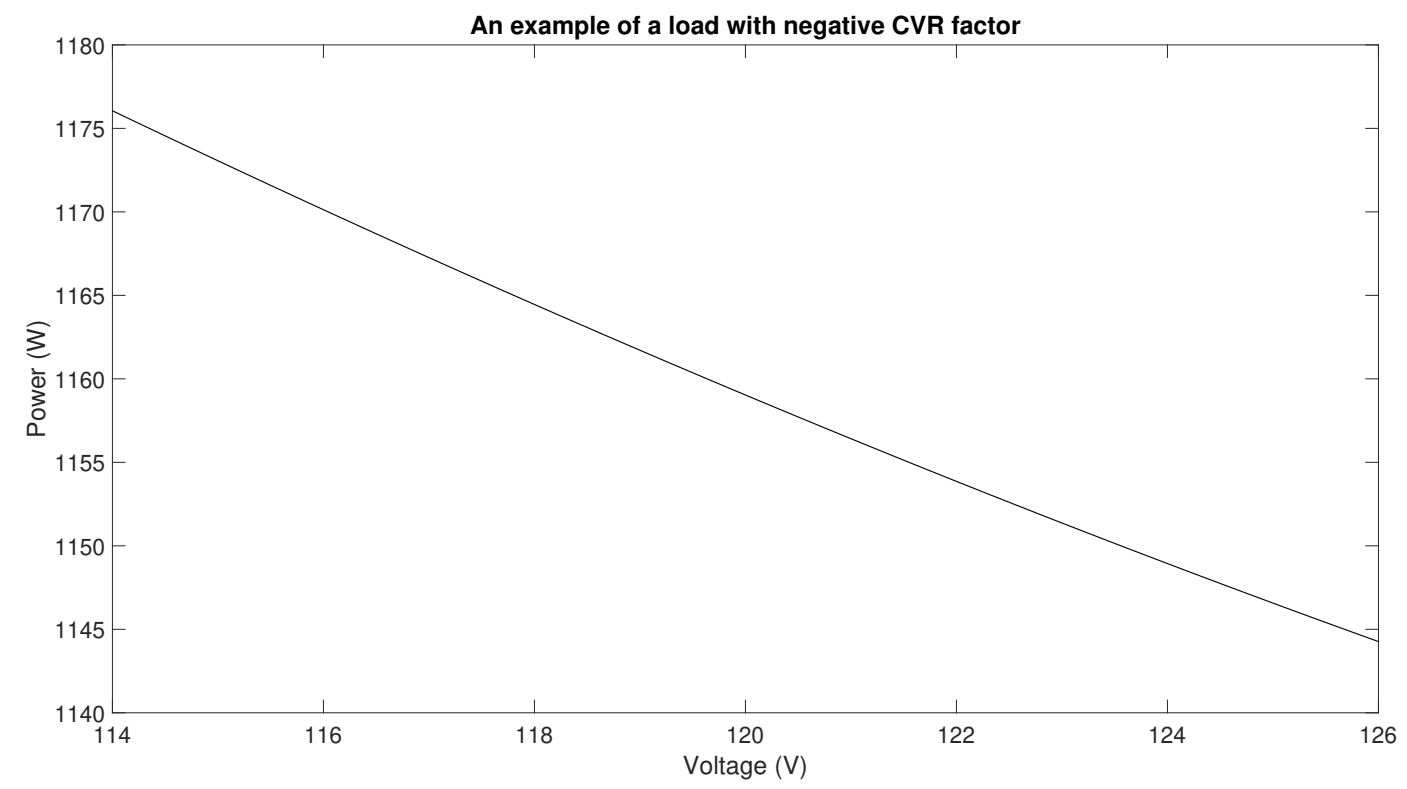

Figure 4.32: An example of the load whose power increases as the voltage decreases.

Figures 4.33 and 4.34 show the simulation results of the RSVC power regulation loop for a load having negative CVR factor. The simulation was performed at an ideal input voltage of $120 \mathrm{~V}$ and $60 \mathrm{~Hz}$. The first plot in Figure 4.33 shows the input, output, and

\footnotetext{
${ }^{2}$ This load is a three-phase compressor load when operated as a single-phase load. The ZIP coefficients used for plotting the real power are given in [34].
} 
reference voltage. The reference voltage changes from $120 \mathrm{~V}$ to $124 \mathrm{~V}$ to reduce the power consumed by the load with a negative CVR factor. Initially, at time $=2$ seconds (the first perturbation), the power regulation loop guesses to decrease the voltage. However, the decrease in voltage causes an increase in the load power-demand, therefore, the power regulation loop corrects itself to change the direction of the perturbation for the next cycle as shown in at time $=4$ seconds. After the second perturbation (at time $=4$ seconds), the power regulation loop keeps working to minimize the power utilized by the load by increasing the reference voltage. However, the reference voltage does not move to $126 \mathrm{~V}$, rather it stops at $124 \mathrm{~V}$ with the output voltage set to $123.5 \mathrm{~V}$. The reason for not being able to increase the load voltage (as well as the reference voltage) all the way to $126 \mathrm{~V}$ is that the duty cycle reaches its lowest limit. Since the inductive power is not required to regulate the voltage (or absorbs VARs), therefore the duty cycle is operating at the minimum allowed value. The second plot in the Figure 4.34 confirms that increases the voltage decreases the power drawn by the load as well as the input power.

The first plot in Figure 4.34 shows the net reactive power generated as the result of the load with negative CVR. It can be seen that the net reactive power output of the RSVC is increasing becoming capacitive as the duty cycle goes lower. The duty cycle finally saturates at $\mathrm{D}=0.1$, therefore preventing any further action from the power regulation. The simulation results show that the RVSC control systems have the ability to counter the loads having positive as well as negative CVR factors.

\subsubsection{Performance of Power Regulation for Combined (Spot) Loads}

Previous sections focused on the power regulation for individual loads. The results shown in this section focus on power regulation for the combination of loads. The control system for power regulation will regulate the power such that the total power for all the loads 

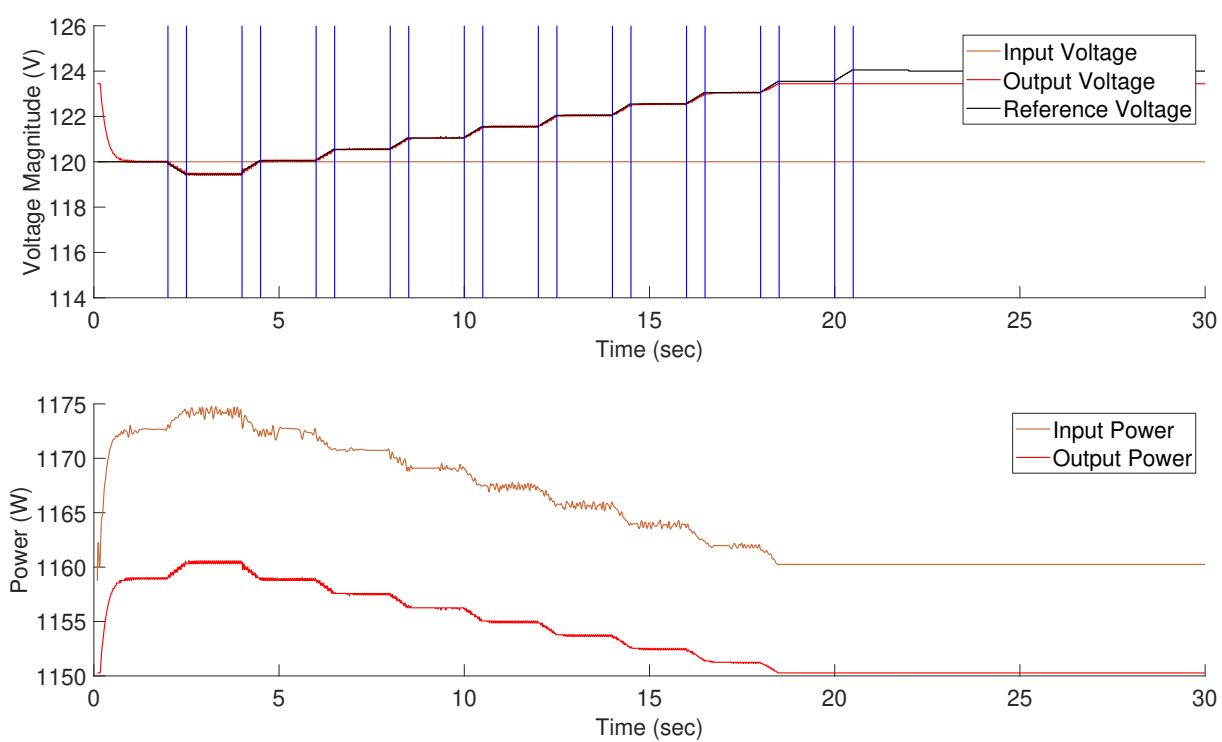

Figure 4.33: Voltages (input, output and reference) and real power (output) change caused by power regulation loop operation for an ideal input voltage with a load having negative CVR factor.
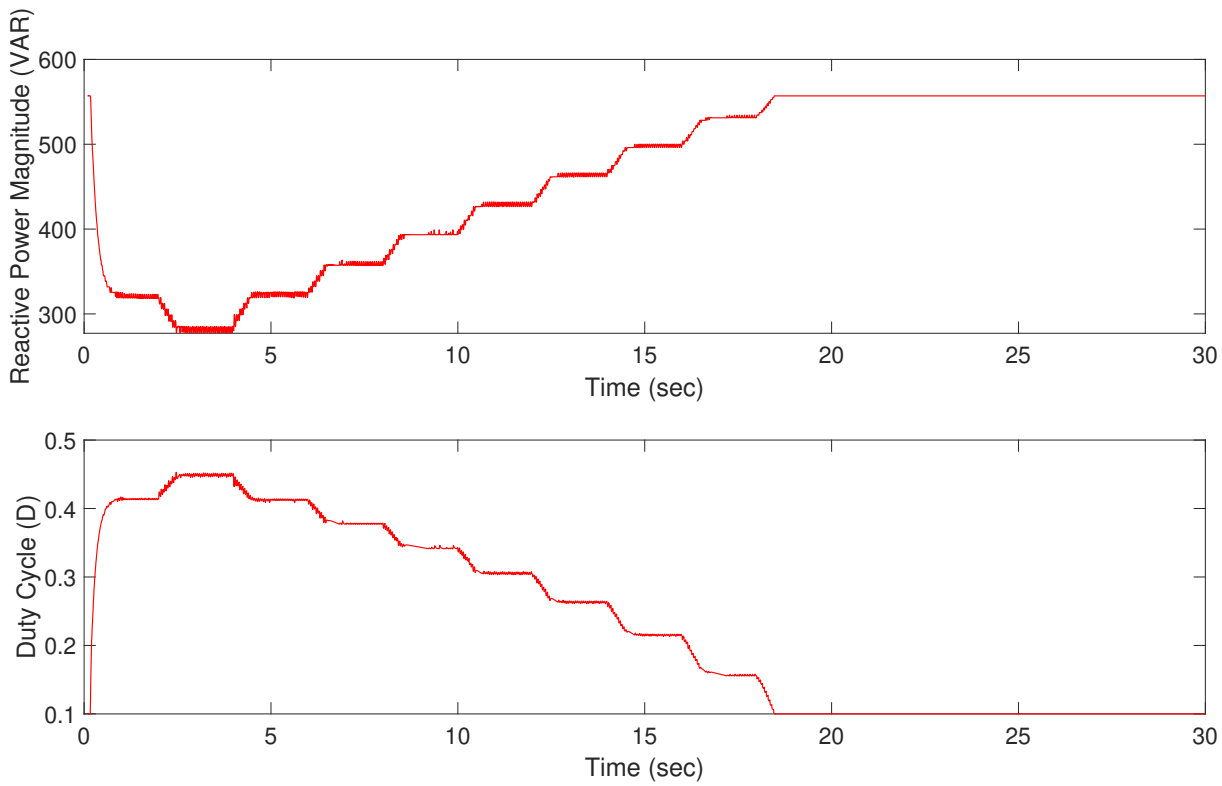

Figure 4.34: Net reactive power and duty cycle change caused by power regulation loop operation for an ideal input voltage with a load having negative CVR factor. 
decreases. Figures 4.35 and 4.36 show the simulation results of the RSVC power regulation loop for a spot load consisting of $2 \mathrm{~kW}$ resistor, a heater and a load with the negative CVR factor. The first plot in the Figure 4.35 shows the input, output, and reference voltage. The reference voltage changes from $120 \mathrm{~V}$ to $114 \mathrm{~V}$ to reduce the total power consumed by the load as shown in the second plot. Similarly, Figure 4.36 shows the variation of the net reactive power provided by the RSVC as well as the variation of the duty cycle as a result of the power regulation control system.
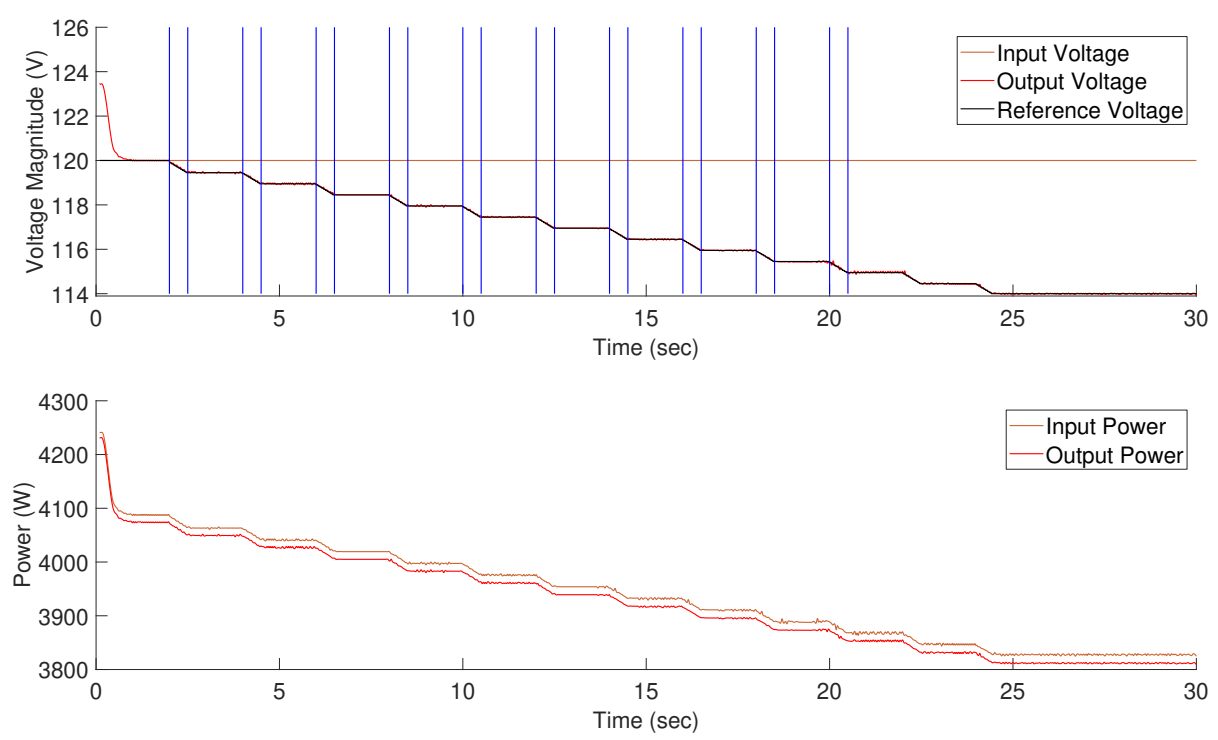

Figure 4.35: Voltages (input, output and reference) and real power (output) change caused by power regulation loop operation for an ideal input voltage with a spot load.

Figure 4.37 shows the change in the total power as well as the change in the power of the individual loads. It can be seen that the power for the loads with positive CVR factor i.e., the $2 \mathrm{~kW}$ resistor and the heater decreases as the voltage decreases whereas the power for the load with the negative CVR increases as the voltage changes from $120 \mathrm{~V}$ to $114 \mathrm{~V}$.

The chapter presents a detailed implementation of the proposed Residential Static VAR Compensator (RSVC). The implementation of open-loop RSVC presented a technological 

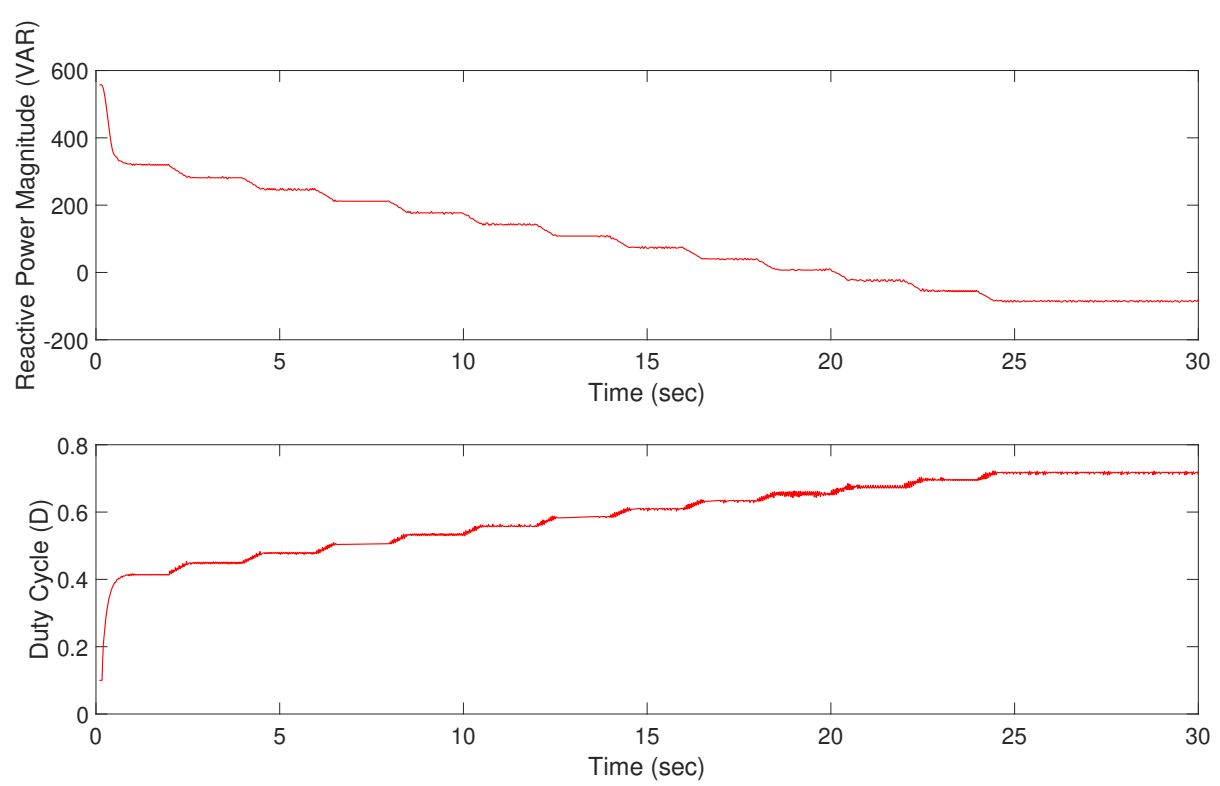

Figure 4.36: Net reactive power and duty cycle change caused by power regulation loop operation for an ideal input voltage with a spot load.
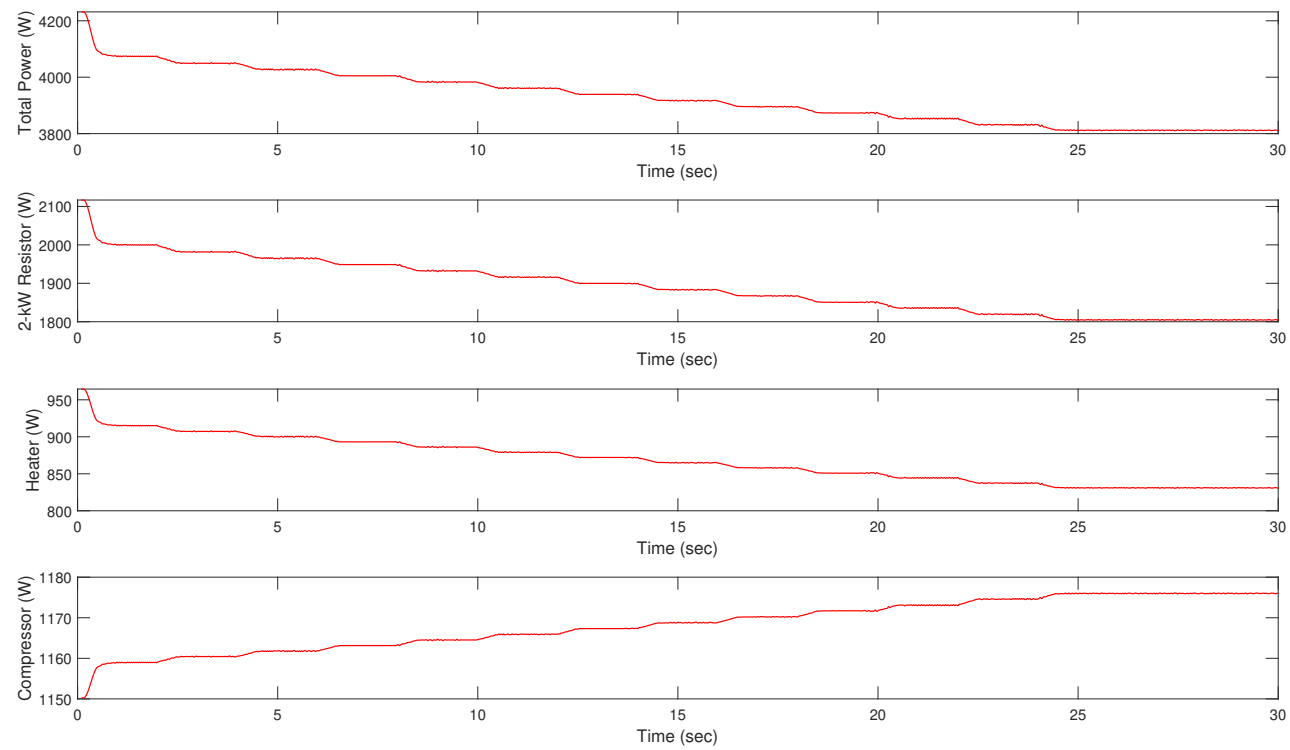

Figure 4.37: Change in power for individual loads as the voltage varies.

superiority over the traditional Static VAR Compensators (SVCs) as the proposed RSVC does not produce low-order harmonics. Moreover, the control systems demonstrated that 
the RSVCs can maintain the line voltage to the desired reference voltage provided that the reactive power required to track the reference voltage is within the reactive power capability of the RSVC. It has also been shown in the simulation that the power regulation loop of the RSVC can track power for the loads with positive as well as negative CVR factors. Such a control system can adapt to the nature of the load and can regulate the power of the loads. It is expected that if RSVCs are embedded in the future distribution feeder (at the customer level), RSVCs can provide a reliable and effective solution to voltage and power stability. Moreover, RSVCs can provide a stable voltage and power in the presence of power generation from renewable resources. 


\section{CHAPTER 5}

\section{DESIGN IMPLEMENTATION ON SOC AND EXPERIMENTAL RESULTS}

In this chapter, a hardware-software co-design and implementation of switching and control signals for the Residential Static VAR Compensator (RSVC) is presented. The power electronics devices used in the development of the RSVC require an accurate switching frequency for the gating signals of the switches. The switching signals used in the RSVC were generated using a Zedboard [65]. The Zedboard is a low-cost development board that contains a Zynq-7000 All Programable System-on-Chip (SoC). The SoC includes the FPGA fabric (programmable logic abbreviated as PL) as well as an ARM-based dual-core Cortex-A9 processor (processing system abbreviated as PS). The on-chip processor is used for high-level programming, while the FPGA programmable fabric is used to create precise gating signals and perform parallel calculations. The tasks in the software and hardware sides communicate with each other via a communication protocol. In Zynq-based system applications, the AXI interface is used as a communication bridge between the PS and the PL.

\subsection{Open-loop SoC Design for RSVC Gating Signals}

The single-phase RSVC device requires two bi-directional switches for its operation. These bi-directional switches operate in a complementary manner to control the reactive power 
of the reactor. The commutation from one switch to another is carried out using a fourstep commutation method based on the input voltage polarity across the bi-directional switches, as explained in the previous chapter. The commutation signals are generated in the programmable logic of the Zynq-7000 System-on-Chip (SoC). This section describes the design methodology used to develop the commutation signals for the bi-directional switches.

Figure 5.1 shows the complete block diagram for generating the switching signals used in the RSVC open-loop design. In the idle/reset state, the state machine stays in the $(\mathrm{SW} 1 \mathrm{~A}, \mathrm{SW} 1 \mathrm{~B}, \mathrm{SW} 2 \mathrm{~A}, \mathrm{SW} 2 \mathrm{~B})=(0,0,1,1)$ state. This means that the switched inductor is disconnected from the power circuit and there is no contribution of the reactive power from the switched inductor. To understand the transition of the state machine from one steady-state to the other, let's assume that the state machine is currently in the (SW1A, $\mathrm{SW} 1 \mathrm{~B}, \mathrm{SW} 2 \mathrm{~A}, \mathrm{SW} 2 \mathrm{~B})=(0,0,1,1)$ state. An up-counter is used to generate the switching frequency for the bi-directional switches. The up-counter increments at every clock cycle until the counter is equal to a desired (maximum) counter value. The maximum counter value represents the switching frequency used for the bi-directional switches. The counter resets once it has reached the maximum value. The overflow (reset) for the counter enables the register, which loads the new duty cycle value into the register. Moreover, the overflow (reset) of the counter triggers the state machine to transition from (SW1A, SW1B, SW2A, $\mathrm{SW} 2 \mathrm{~B})=(0,0,1,1)$ to $(\mathrm{SW} 1 \mathrm{~A}, \mathrm{SW} 1 \mathrm{~B}, \mathrm{SW} 2 \mathrm{~A}, \mathrm{SW} 2 \mathrm{~B})=(1,1,0,0)$. The comparator is used to compare the value of the counter with the duty cycle. The comparator generators a trigger signal for the state machine when the counter value is equal to the duty cycle. The trigger signals cause the state machine to transition from the (SW1A, SW1B, SW2A, SW2B) $=$ $(1,1,0,0)$ state to the (SW1A, SW1B, SW2A, SW2B) $=(0,0,1,1)$.

A Pmod rotary encoder [66] from Digilent ${ }^{\circledR}$ is used for controlling the duty cycle of 


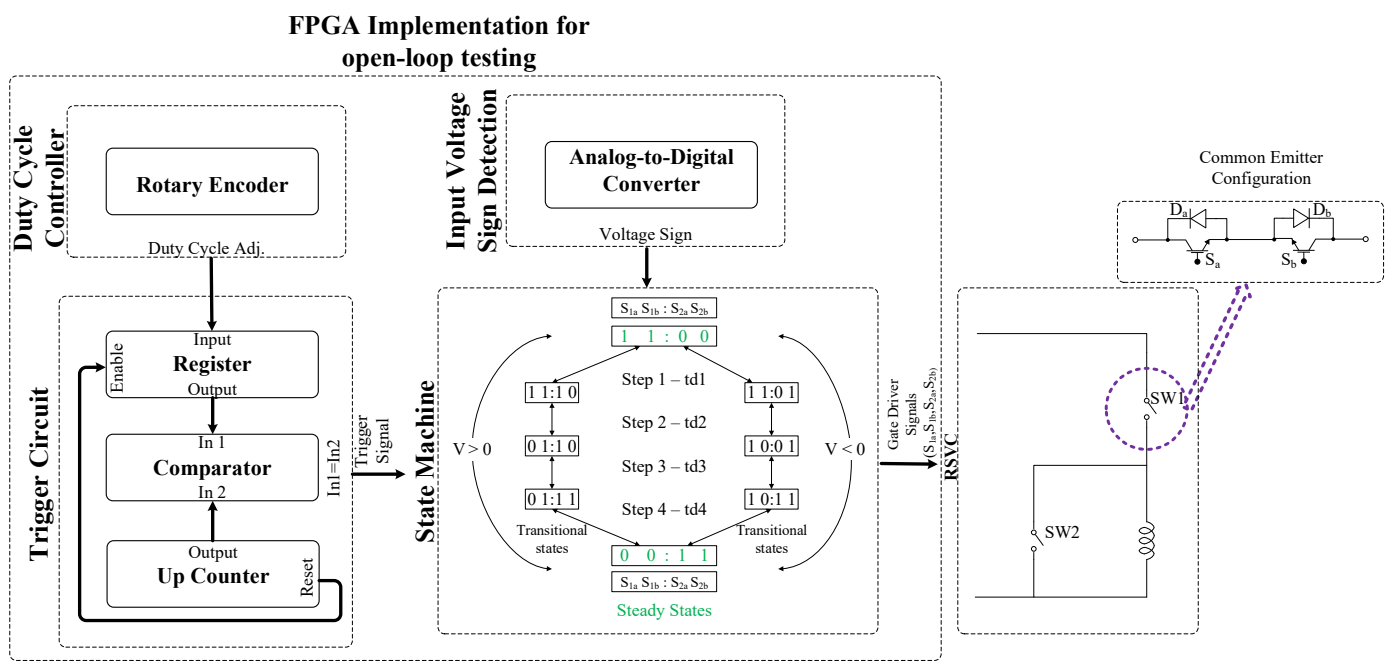

Figure 5.1: Complete block diagram for System-on-Chip implementations for generating the gating signals.

the top bi-directional switch. The 24-bit input from the rotary encoder is loaded into the hardware register when the enable (load) signal is high. This hardware register holds the information for the duty cycle of the top bi-directional switch. At every positive edge of the clock, the value in the register is compared with the up-counter. When the counter value is equal to the input of the rotary encoder, meaning the inductor has been connected to the power circuit for the desired time of the switching period, a trigger signal is set high. This signal causes the state machine to transition from one steady-state to the other steady-state.

The commutation from one switch to another involves a change in the state of four switching devices. The switching devices have finite turn-on and turn-off times. It is essential to provide sufficient time delay between the transitional states to avoid shortcircuiting the AC input. The time delay $t_{d}$ used between the transitional states for the RSVC prototype is set to $1 \mu \mathrm{sec}$. Figure 5.2 shows the complete laboratory prototype of the RSVC developed at Boise State University. Table 5.1 shows the values of the RSVC components used in the development of the RSVC prototype. 


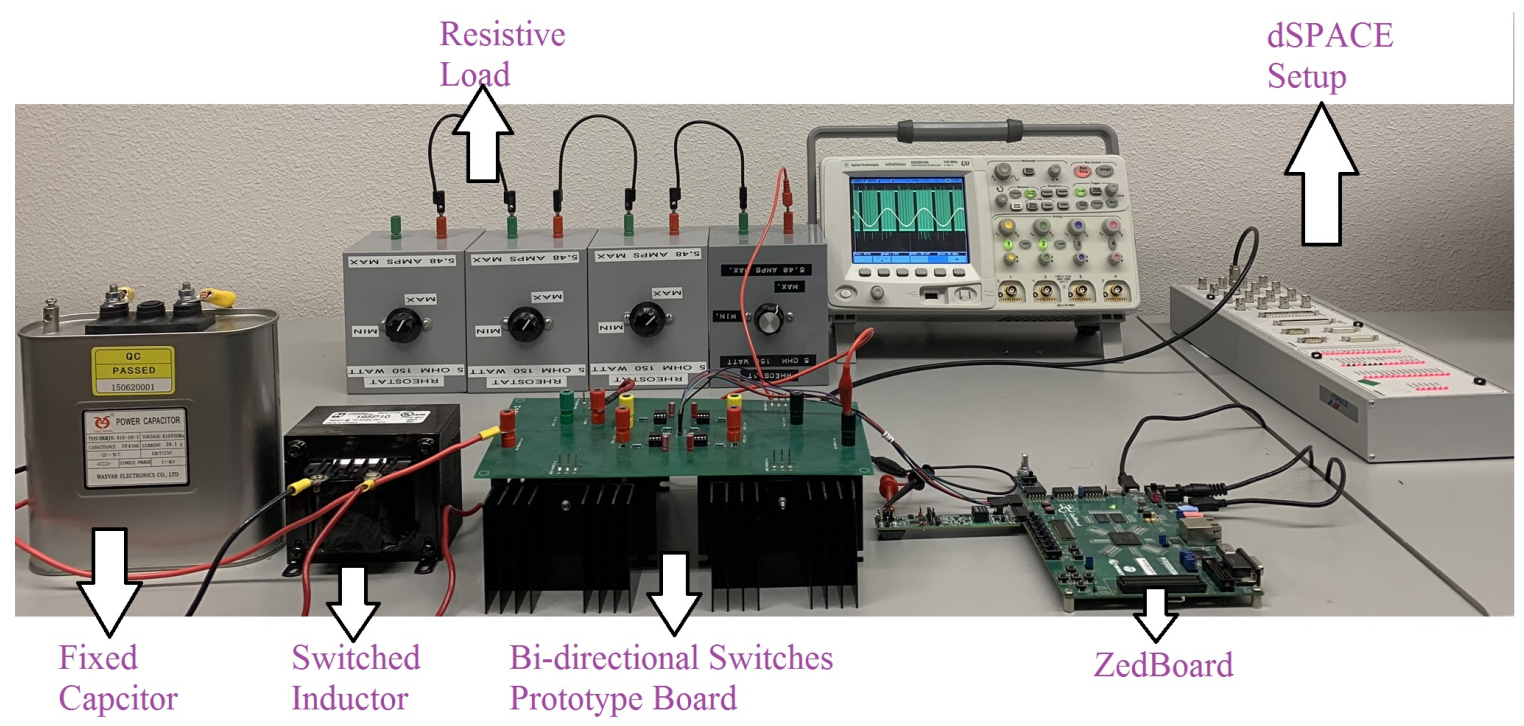

Figure 5.2: Laboratory prototype of Residential Static VAR Compensator (RSVC).

Table 5.1: RSVC components used in the laboratory prototype.

\begin{tabular}{|c|c|c|}
\hline Component Name & Description & $\begin{array}{c}\text { Value/ } \\
\text { Datasheet }\end{array}$ \\
\hline \hline Power Capacitor & $\begin{array}{c}\text { Fixed capacitor rated for 415 V } \\
10 \text { kVAR (single-phase capacitor) }\end{array}$ & $190 \mu \mathrm{F}$ \\
\hline Switched Inductor & Power Inductor rated for 10 A & $30 \mathrm{mH}$ \\
\hline $\begin{array}{c}\text { Switching Devices } \\
\text { bi-directional switch }\end{array}$ & IRGP4066DPBF [67] \\
\hline $\begin{array}{c}\text { Analog-to-Digital (ADC) } \\
\text { Device }\end{array}$ & $\begin{array}{c}\text { Used for input voltage sign } \\
\text { detection in open-loop RSVC }\end{array}$ & {$[68]$} \\
\hline
\end{tabular}

\subsection{Open-loop Experimental Testing Results}

\subsubsection{Input and Output Voltage}

Figure 5.3 shows the experimental results of the input (source) voltage and the output (load) voltage. The input and output voltage waveforms are recorded when the input voltage is 60 Vrms and the duty cycle of the top bi-directional switch is equal to $50 \%$. It can be seen that 
the input and output voltages remain sinusoidal without short-circuit during the operation.
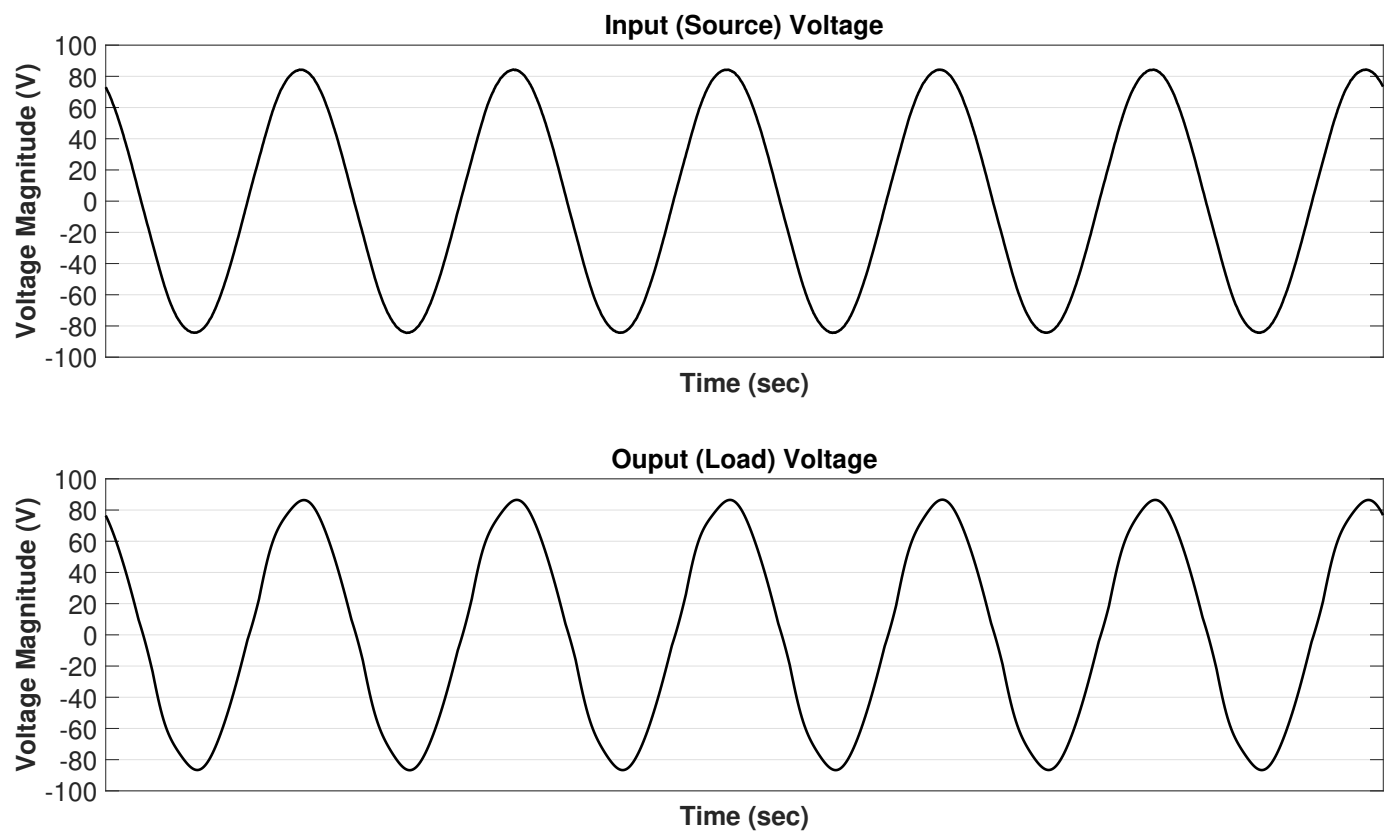

Figure 5.3: Experimental result for input (source) and output (load) voltage waveforms at duty cycle $\mathrm{D}=0.5$.

\subsubsection{Input (Source) Current}

Figure 5.4 shows the input (source) current for the proposed configuration of the RSVC. The source current remains sinusoidal since the PWM-based switching of inductor does not produce low-order harmonics. The waveform in Figure 5.4 is slightly distorted due to the halting of the commutation near the zero-crossing of the input voltage. The halt in commutation signals is suggested to avoid the false detection of the input voltage signal. However, for a single-phase device, the input phase voltage is measured with reference to the voltage of the neutral point. Therefore, in the case of a falsely detected sign of voltage across the bi-directional switches (near zero-crossing), the switching devices are stressed with a short-circuit current during the period of a time delay, which is equivalent to a delay 
in the commutation sequence step $t_{d}$. In most cases, the transitional time period is around 1 to $2 \mu \mathrm{sec}$, which is within the thermal properties of the switching devices. Hence, the prevention of the commutation, near the zero-crossing, can be made very small to avoid the distortion of the input current. In the experiments, the commutation was halted when the input voltage was between $\pm 10 \mathrm{~V}$.

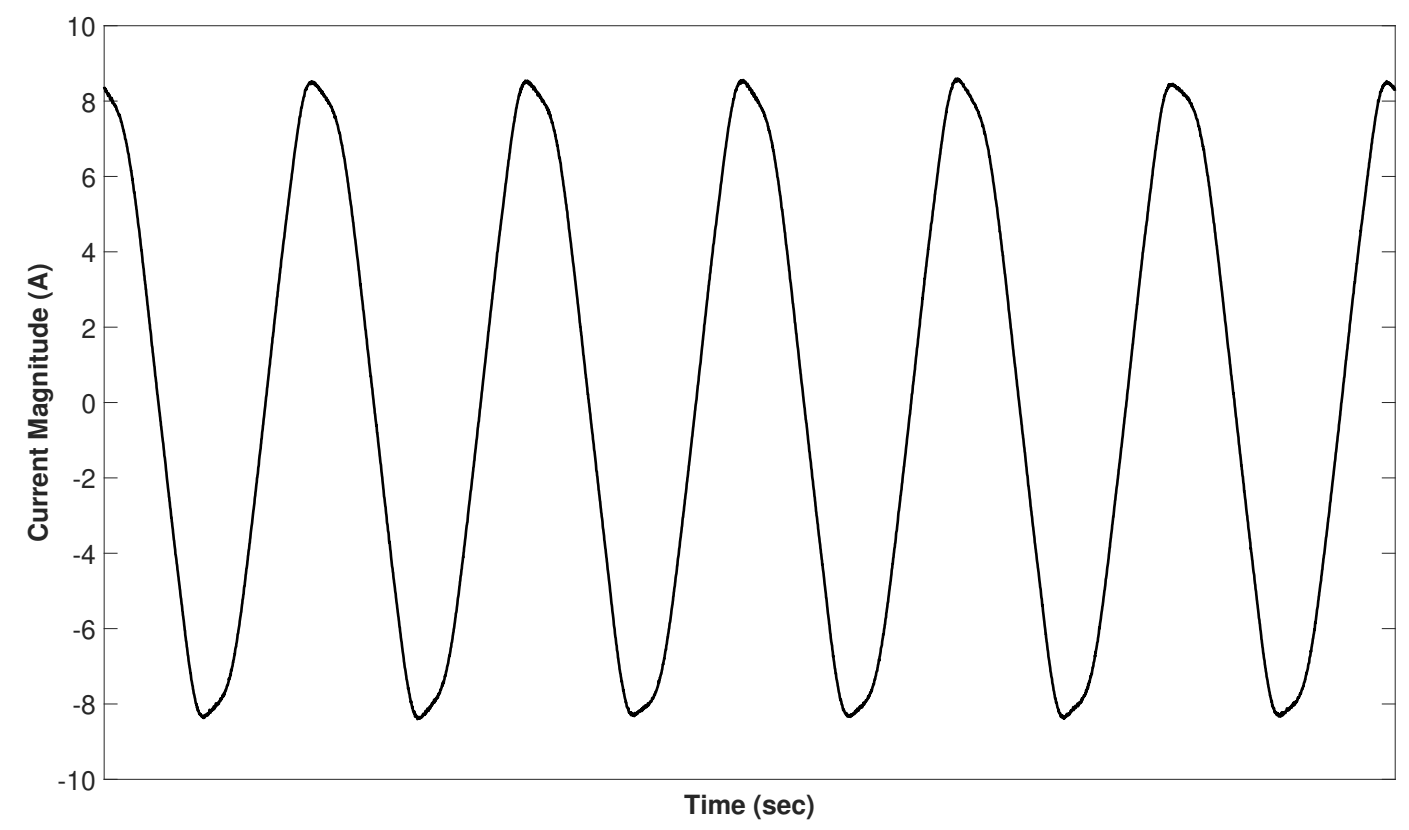

Figure 5.4: Experimental result for input (source) current at duty cycle $D=0.5$.

Figure 5.5 shows the harmonic content of the source current. The plot shows the harmonics content of the fundamental $\left(1^{\text {st }}\right)$ component of the source current at $60 \mathrm{~Hz}$, as well as the harmonic content for the multiples of the fundamental frequency with respect to the fundamental component. Since the source current is fairly sinusoidal, the fundamental component of the source current dominates the harmonic spectrum. The plot also reports the peak harmonic values for different harmonics. The calculated total harmonic distortion for the source current is approximately equal to $3.43 \%$. 


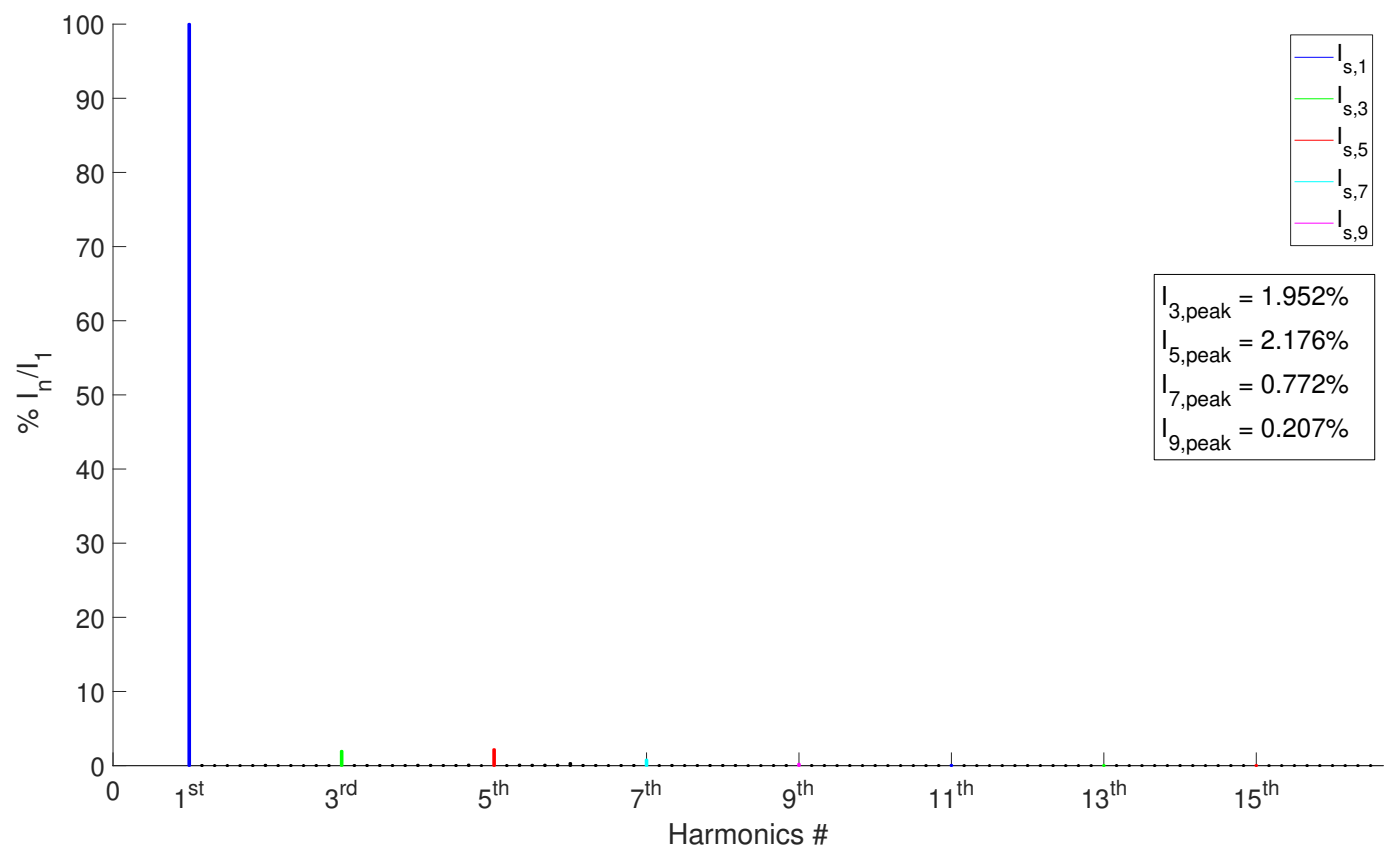

Figure 5.5: Harmonic analysis of the input (source) current.

\subsubsection{Reactor Current}

Figure 5.6 shows the current for the switched reactor in the RSVC at duty cycles of 0.25 , 0.50 and 0.75 respectively. The harmonic analysis of the reactor current at those duty cycles is also shown in the figure. The reactor current increases as the duty cycle increases. Increasing the duty cycle introduces the reactor into the power circuit for an increased amount of time in the switching period. The harmonic analysis of the reactor current at different duty cycles confirms that the reactor current remains sinusoidal at all duty cycles.

\subsubsection{Current through the bi-directional switch}

Figure 5.7 shows the current through the top bi-directional switches. This current is a chopped envelope of the reactor current. It can be seen that the current through the top bi-directional switch increases as the inductor current increases with the duty cycle. The 

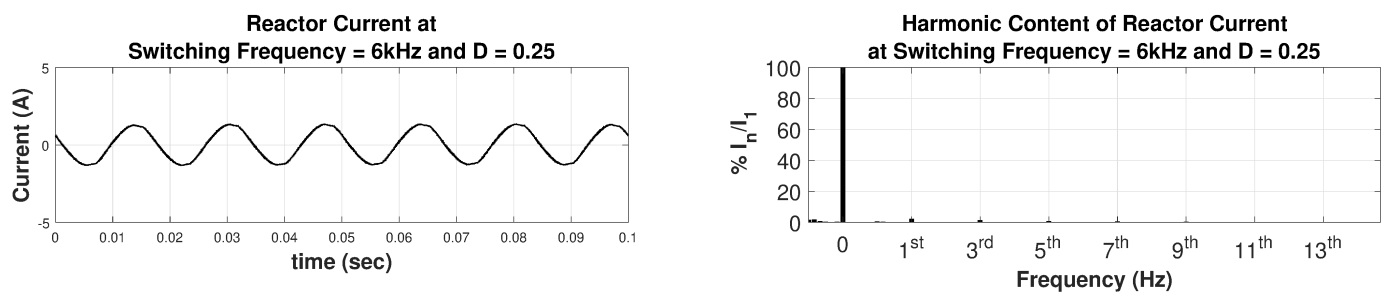

Reactor Current at

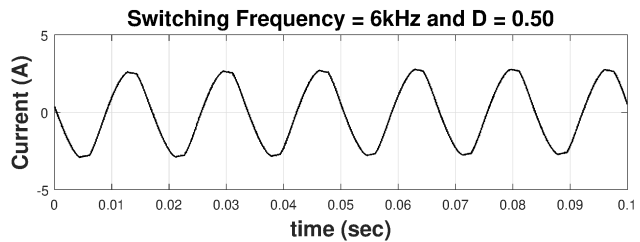

Harmonic Content of Reactor Current

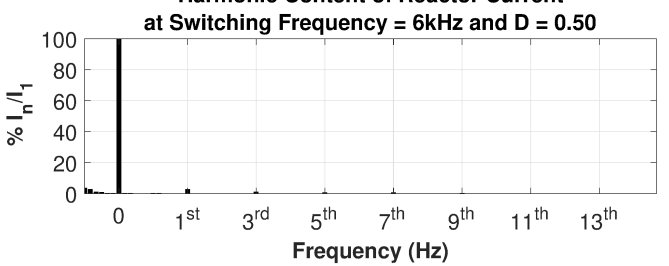

Reactor Current at
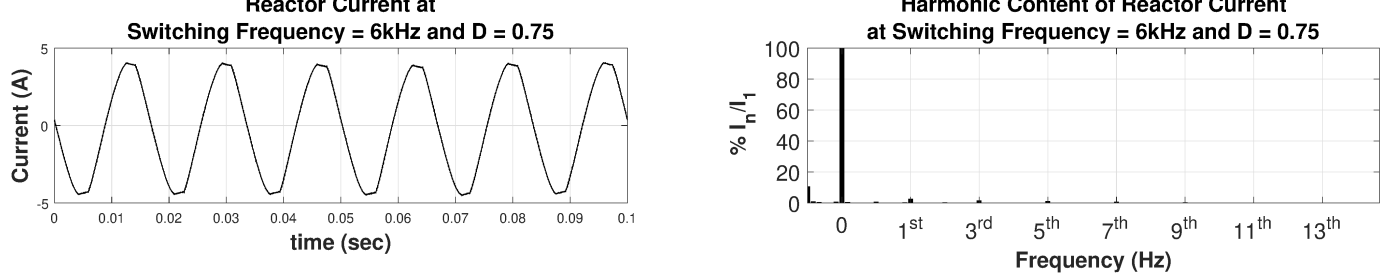

Figure 5.6: Experimental result for inductor current at different duty cycles.
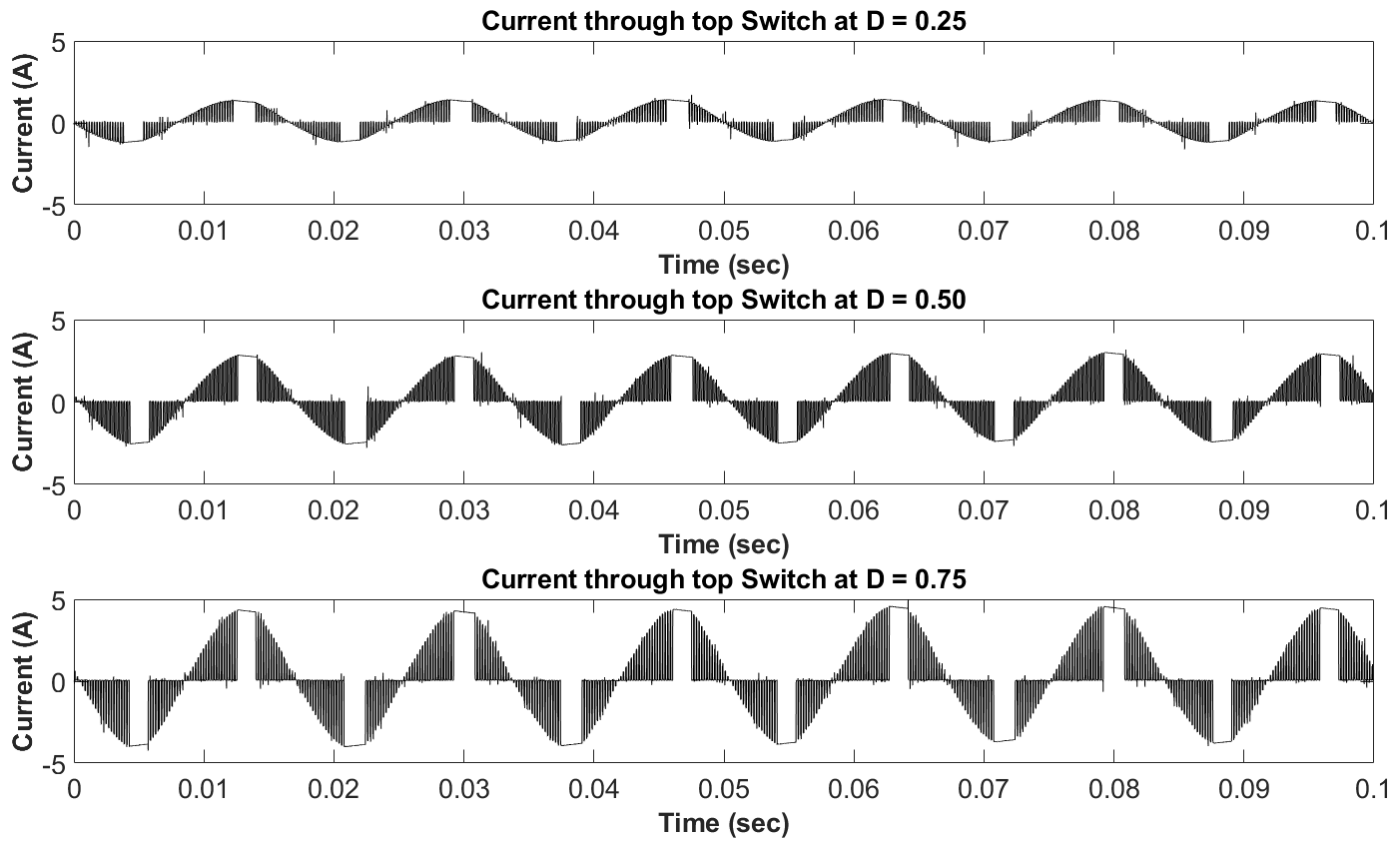

Figure 5.7: Experimental result for current through top switch at different duty cycles. 
area where the commutation is halted, near the zero-crossing of the voltage, can be seen in the reactor current waveform. Therefore, a path for the flow of current always exists and the reactor current remains sinusoidal.

\subsubsection{Output (Load) Voltage Variation with Duty Cycle}

Figure 5.8 shows the variation in the output (load) voltage with the variation in the duty cycle. The experiment was carried out with an input voltage of $60 \mathrm{Vrms}$ at $60 \mathrm{~Hz}$ frequency. When the duty cycle is 0 , meaning the top switch connecting the reactor to the power circuit is off, the output voltage is at 73.9 Vrms. The increase in the voltage is due to the presence of a fixed capacitor in the RSVC circuit. As the duty cycle increases, the inductor is connected to the power circuit, depending on the turn-on time specified by the duty cycle. When the duty circle reaches 1 , the inductor is connected to the power circuit (or the regulation bus) for the complete switching period. It can be seen that the output voltage decreases with the duty cycle. By changing the duty cycle, it is possible to control the output voltage. The experiment was repeated at different switching frequencies, as shown in the figure, to verify the operation of the RSVC prototype.

\subsubsection{Input and Output Power Variation with Duty Cycle}

Figure 5.9 shows the variation of the input and output power with a change in the duty cycle. The experiment was carried out with an input voltage of $60 \mathrm{Vrms}$ at $60 \mathrm{~Hz}$ frequency. The output voltage is measured across a $20 \Omega$ resistive load. When the duty cycle is 0 , the output power is equal to $300 \mathrm{~W}$, whereas the input power is equal to $320 \mathrm{~W}$. As the duty cycle increases, the output voltage decreases. For a resistive load, the decrease in output voltage means a lower output power. This trend can be seen in Figure 5.9. As the duty 


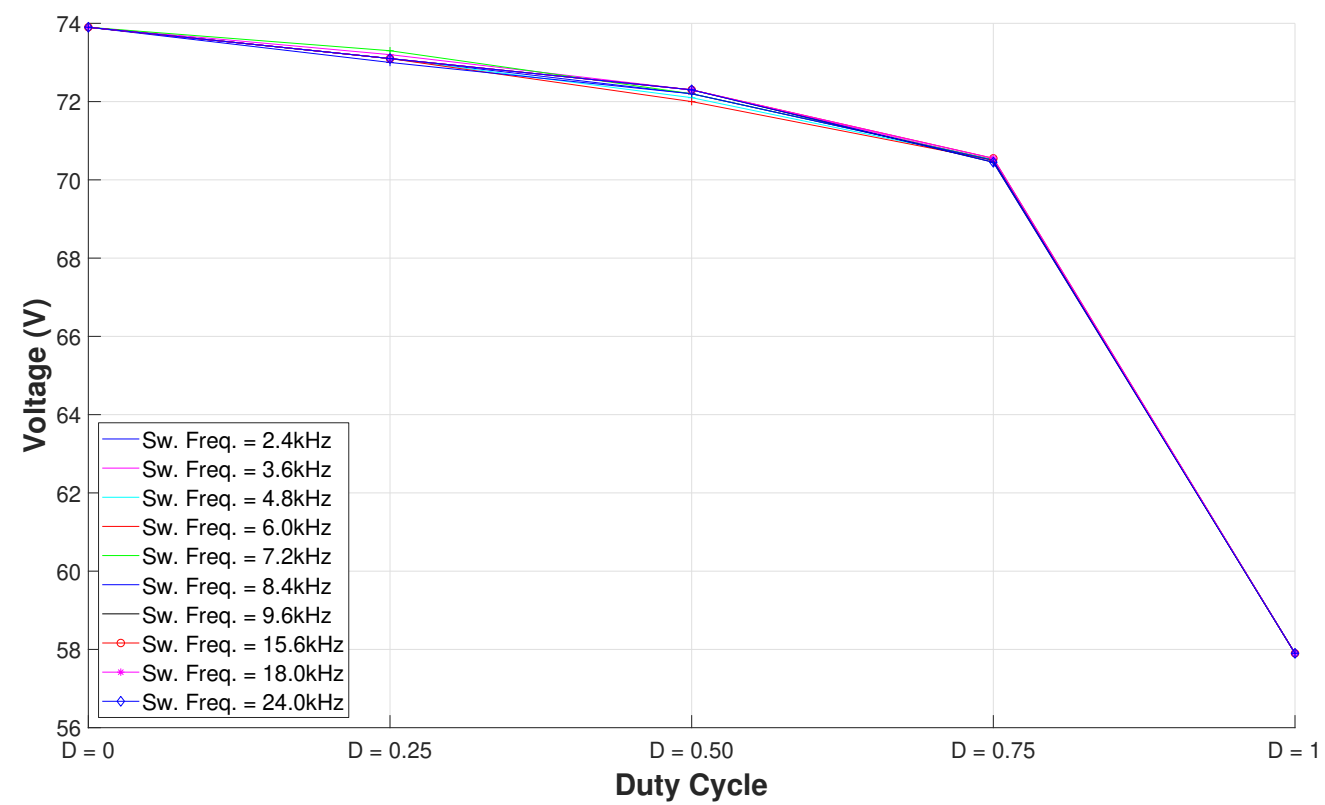

Figure 5.8: Experimental results for output (load) voltage variation with duty cycle (D).

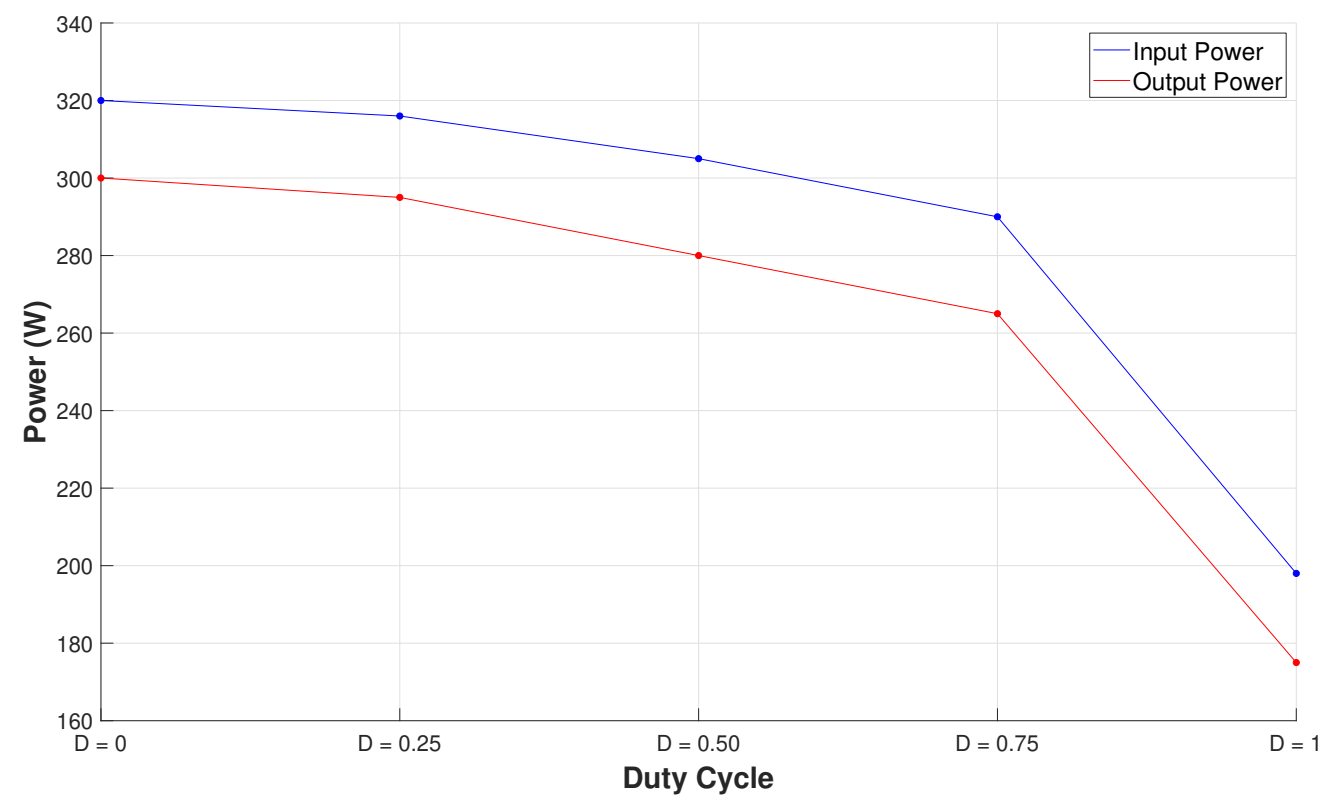

Figure 5.9: Experimental results for input and output power variation with duty cycle (D). 
cycle increases, the output power goes down. As the load demand power decreases with an increase in the duty cycle, the input power at the feeder header also decreases.

\subsection{Closed-loop Design for RSVC}

The laboratory prototype for the closed-loop design of the RSVC consisted of using the Zedboard in conjunction with a dSPACE DS1104 platform. This setup enabled rapid prototyping of the control systems for the RSVC. Figure 5.10 shows the complete schematic of the RSVC control system implementation. The Zedboard is used to generate the gating signals for the bi-directional switches whereas the dSPACE platform is used to build the two control loops, namely the voltage and the power regulation control loops, that are essential for the closed-loop operation of the RSVC. The objective of the voltage regulation control loop is to implement a quick-response inner voltage regulation, such that the output load voltage can track the desired reference voltage. And the goal of the outer slower-response power regulation loop is to regulate the power of the loads connected to the regulation bus, such that minimum power is utilized during their operation.

For generating the switching signals, the ADC value of the input voltage is used to detect the polarity of the input voltage. The output signal from the ADC channel is fed to the Zedboard for producing proper switching signals. Similarly, the load voltage and load current are required for the calculations that are involved in the control loops. The stepped down values of these signals are provided to dSPACE which takes these signals as input to perform the calculation required for voltage and power regulation loops. After performing the calculation, the dSPACE generates the duty cycle, which is used by the Zedboard to generate the switching signals accordingly. 


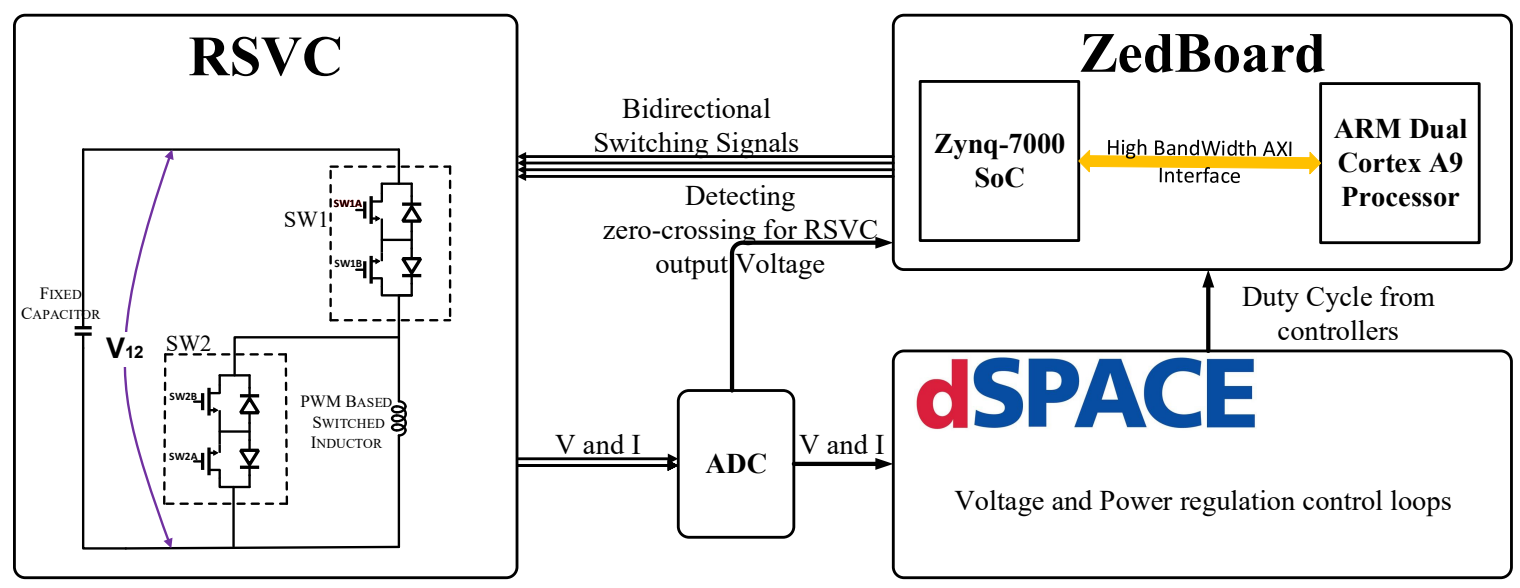

Figure 5.10: Complete block diagram for closed-loop RSVC control system design implemented in the Zedboard and dSPACE platform.

\subsubsection{SoC Implementation}

Figure 5.11 shows the block diagram for the RSVC control signals implemented in the Zynq-7000 FPGA. The Intellectual Property (IP) block presented is the core of this design. This IP block generates the four switching signals that are used for the commutation of the two bi-directional switches. The internal structure of this IP block is similar to the block diagram in Figure 5.1.

The Zynq-7000 System-on-Chip integrates a high performance Processing System (PS) with Programmable Logic (PL) and the AXI interface is used as a communication bridge between the PS and PL. For the development of the RSVC control system, the processing system (PS) part of the Zedboard is equipped with a timer interrupt. This timer interrupt is configured to occur at every cycle of the AC mains frequency. This interrupt enables the programming logic (PL) to read the duty cycle from the dSPACE via an AXI interface. The updated duty cycle is used to control the amount of inductive reactive power provided by the RSVC. In other words, the inner voltage regulation control loop is updated at every cycle of the input voltage frequency to control the net reactive power of the RSVC. 


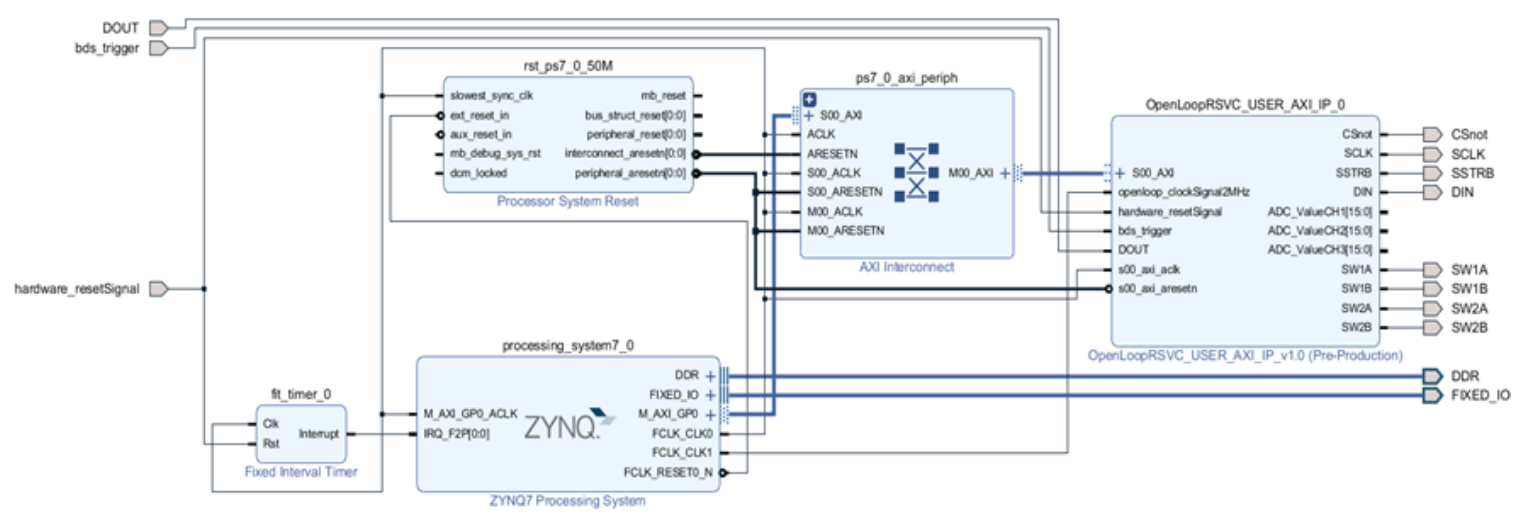

Figure 5.11: Zynq-7000 SoC implementation of the state machine on processing logic (PL) and the timer interrupt on processing system (PS) for the development of the closed-loop RSVC on Zedboard.

\subsection{Design of Voltage Regulation Control Loop}

Figure 5.12 shows the voltage regulation loop implemented in dSPACE. The control system calculates the RMS value of the load voltage which is then compared to the desired reference voltage. The error voltage is fed to the PI controller, which generates the duty cycle of the bottom bi-directional switch. This duty cycle is used to calculate, (1-D), to generate the duty cycle of the top bi-directional switch.

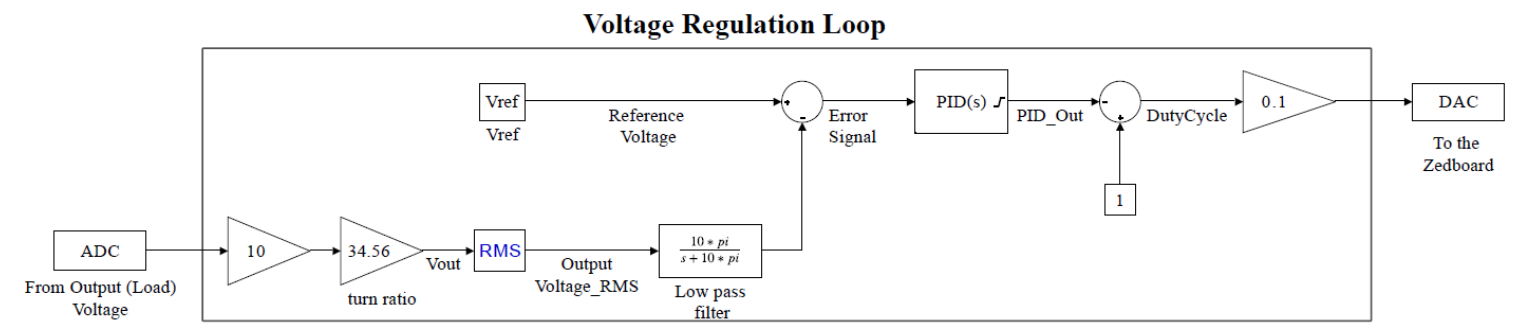

Figure 5.12: Voltage regulation loop implemented in Simulink/dSPACE. 


\subsubsection{Tracking the Reference Voltage $V_{r e f}$}

Figure 5.13 shows the result of the testing of the voltage regulation loop. The first plot in the figure shows the tracking of the output voltage as the reference voltage changes. The reference voltage is varied by using the output from the staircase generator in Simulink. The output from the staircase generator is added to the original reference voltage of 36.5 V. After the initial startup, the voltage regulation control system successfully tracks the original reference voltage. At 30 seconds, the reference voltage is suddenly changed to $38 \mathrm{~V}$. The spike in the error signal, as shown in the second plot, causes the PI controller to adjust the output voltage such that the error (the difference between the output (load) voltage and the reference voltage) goes to zero. Since the reference voltage is now higher than the output load voltage, the duty cycle must decrease so that the net reactive power, $Q_{S V C}=Q_{C}-Q_{L}(D)$, provided by RSVC is higher compared to the case when the reference
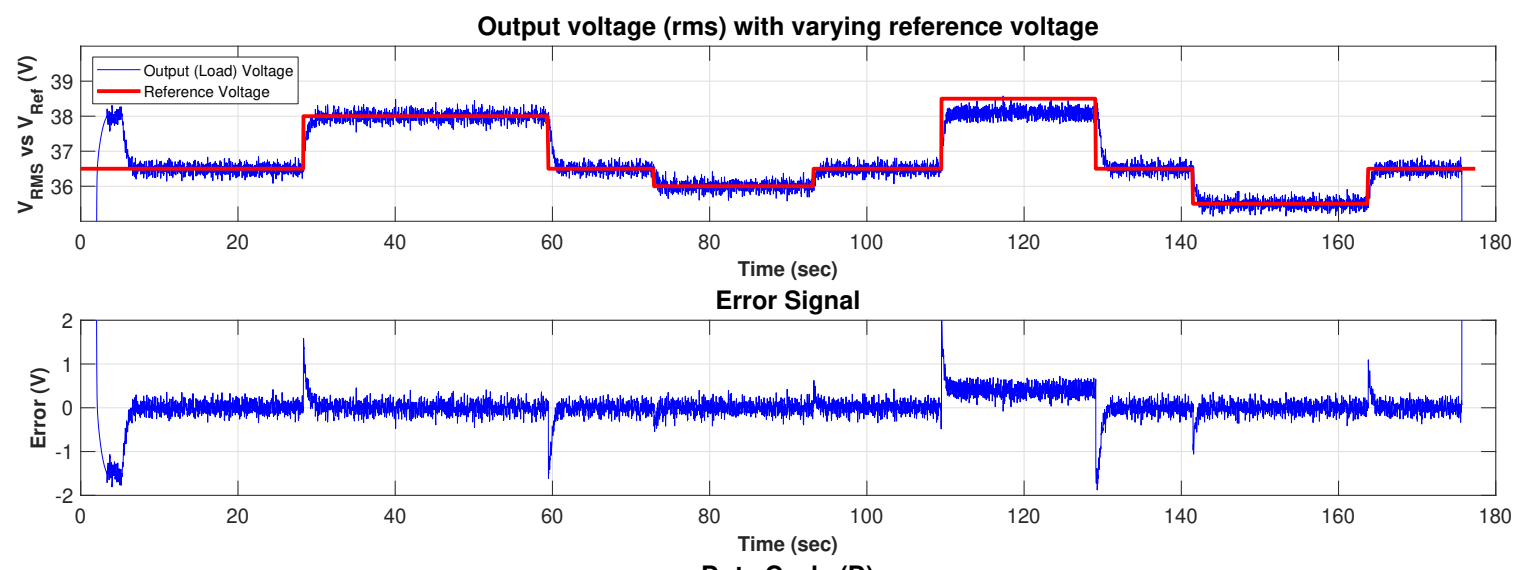

Duty Cycle (D)

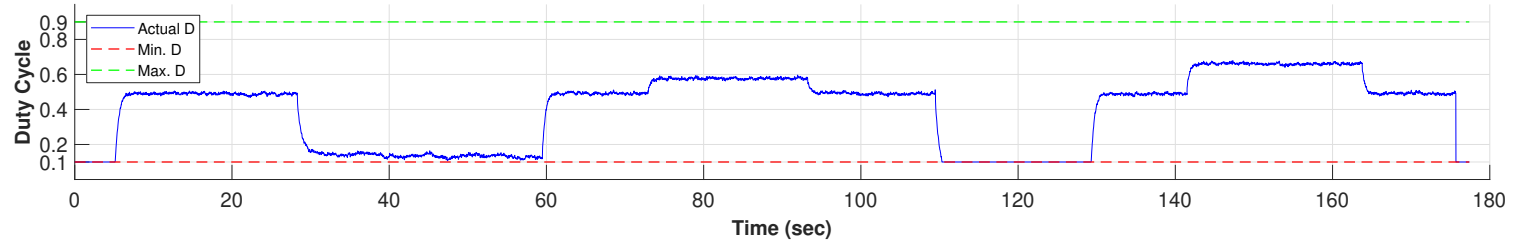

Figure 5.13: Plots of RSVC laboratory prototype with voltage regulation loop test results. 
voltage was $36.5 \mathrm{~V}$. This fact can be verified by the third plot of Figure 5.13. The plot shows the duty cycle decreases from 0.4 to 0.1 , so that the output load voltage is equal to the reference voltage.

An interesting scenario arises at 110 seconds. At this time, the reference voltage is changed to $38.5 \mathrm{~V}$. The control system reacts to this change and decreases the duty cycle. However, the output (load) voltage does not reach the reference voltage level. This is due to the fact that the control system does not produce a duty cycle lower than 0.1 . The limitations on the minimum and maximum duty cycle were set during the testing of the RSVC to avoid the generation of the duty cycle for very short intervals. In a real-world application, the duty cycle can be varied from $0 \%$ to $100 \%$.

Figure 5.13 also shows the reactive power limitation of the RSVC. In the case, when reactive power requirements exceed the RSVC reactive power capability, the fixed capacitor and switched inductor can be replaced with the appropriate values. This makes the RSVC a scalable device that can be used at different locations, according to the reactive power requirements.

The voltage regulation control system presented can also be used to counter the variability and unpredictability associated with distributed energy resources, especially for those that use renewable fuel for power generation. The results presented establish the effectiveness of the closed-loop RSVC for voltage support and for shaping the load demand to follow a certain reference voltage, as desired. The experiments carried out confirm the scientific theory presented in the dissertation, as well as the effectiveness of the closed-loop RVSC in supporting the input (source) voltage of an unstable power system in a way which is different from switched capacitors, fixed capacitors and dynamic capacitors (D-CAP). 


\subsection{Design of Power Regulation Control Loop}

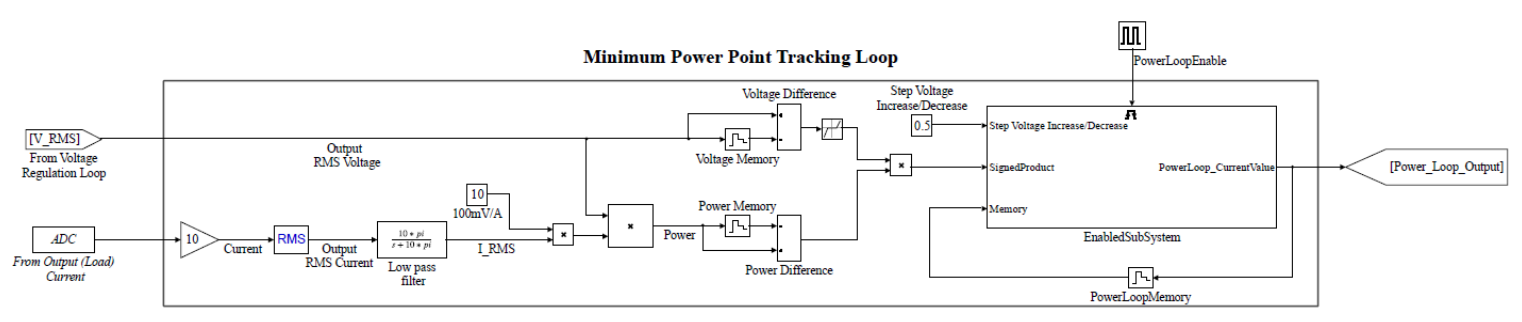

Figure 5.14: Power regulation loop implemented in Simulink/dSPACE.

Figure 5.14 shows the power regulation loop for the RSVC laboratory prototype. A current transducer is used to measure the load current. The output from the current transducer is multiplied by a gain of 10 to obtain the actual RSVC output current. The power regulation loop calculates the average power, which is used to sort the direction of the power regulation loop. The RSVC average power is obtained by multiplying the RMS voltage and the RMS current. When compared with implementation in Section 4.6, the power regulation loop in the RSVC laboratory prototype has a similar structure. In the simulation, however, the reference voltage is increased or decreased linearly i.e., the reference voltage is adjusted in 30 cycles (or 0.5 seconds) with a 0.05 change in reference voltage and with a perturbation period every 2 seconds. In the implemented control loop, each perturbation period is 5 seconds and the reference voltage is changed by $\pm 0.5 \mathrm{~V}$. The complete control system implementation in dSPACE, including the power and voltage regulation loops, is shown in Figure 5.15.

\subsubsection{Tracking the Power for the Resistive Load}

The testing of the complete RSVC control system was performed using a dSPACE platform with the Zedboard. The performance of the power regulation loop in the RSVC laboratory 


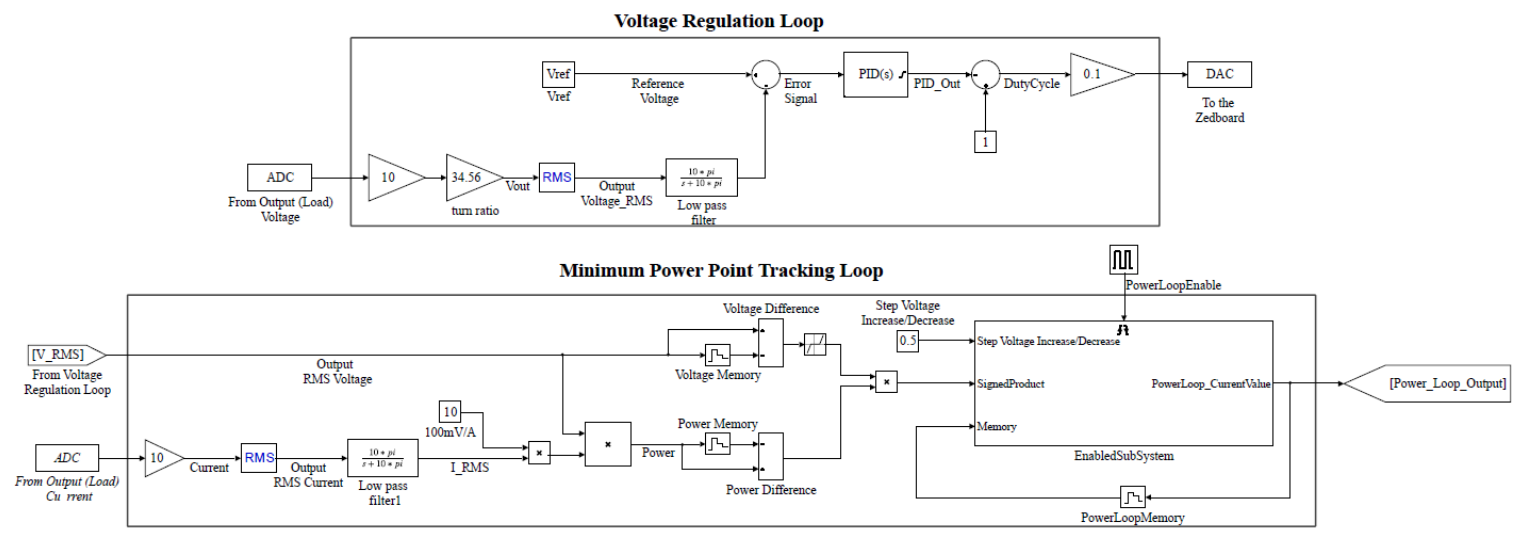

Figure 5.15: Complete control system for an RSVC laboratory prototype in Simulink/dSPACE.

prototype is shown in Figures 5.16 and 5.17. A $20 \Omega$ resistor was connected to the RSVC as a purely resistive load. For a purely resistive load, a lower voltage means a lower output power. Therefore, the power regulation loop should regulate the RSVC voltage to the minimum voltage, which corresponds to the maximum duty cycle $(0.9)$ for the top bidirectional switch. The power regulation loop updates less frequently than the voltage regulation loop. In the prototype, the power regulation control systems update the reference voltage every 5 seconds, whereas the voltage regulation control system updates at every cycle of the input voltage. The reference voltage is increased or decreased by $0.5 \mathrm{~V}$ as a result of the output from the power regulation loop.

Figure 5.16 shows the result of the testing of the power regulation loop. The first plot in the figure shows the change in the reference voltage as a result of output from the power regulation loop. Since the load being tested is a purely resistive load, the output from the power regulation loop results in $-0.5 \mathrm{~V}$ every at every 5 -second interval. The second plot in the figure shows the adjusting of the duty cycle as a result of the change in the reference voltage. As the output (load) voltage needs to be decreased (because of the 
resistive load), the duty cycle of the top bi-directional switch should increase. The voltage keeps decreasing until the duty cycle reaches the maximum limit i.e., when $\mathrm{D}=0.9$.

During the 30 to 35 seconds interval, the output of the power regulation loop changes from $34 \mathrm{~V}$ to $33.5 \mathrm{~V}$. However, the duty cycle had already reached its upper limit (0.9). Therefore, the output load voltage does not track the reference voltage. The experiments were carried out to test the range of the duty cycle from 0.1 to 0.9 . However, in a realworld application, the duty cycle will be limited, so that the output load voltage does not go beyond the ANSI C84.1 voltage limits. Once the voltage limit is reached, the power regulation stays at that level without further decreasing the reference voltage.
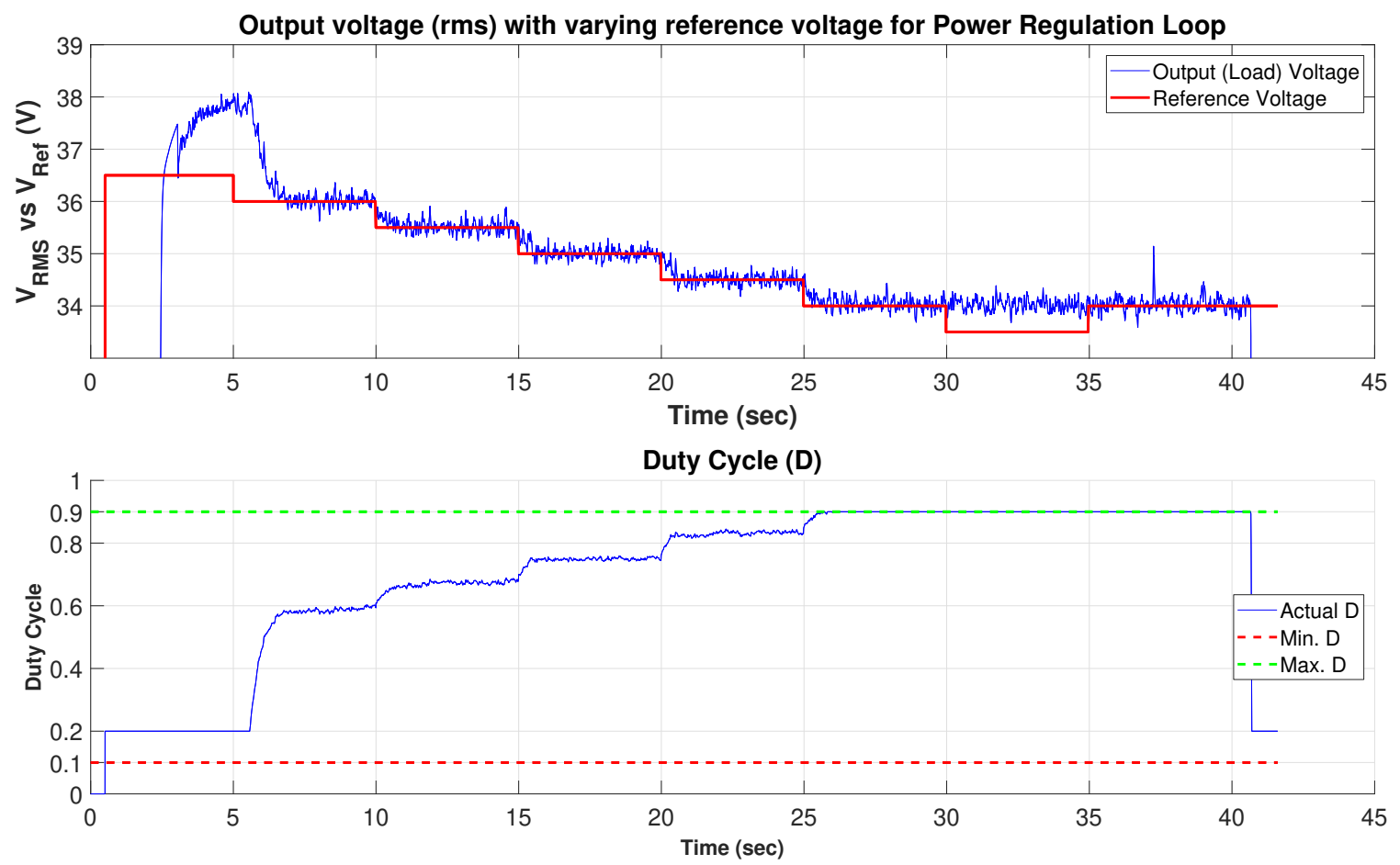

Figure 5.16: Plots of RSVC laboratory prototype with voltage regulation loop test results.

The first plot in Figure 5.17 shows the output power for the resistive load. The output power decreases from $72 \mathrm{~W}$ to $58 \mathrm{~W}$ as a result of the decrease in the output (load) power. 

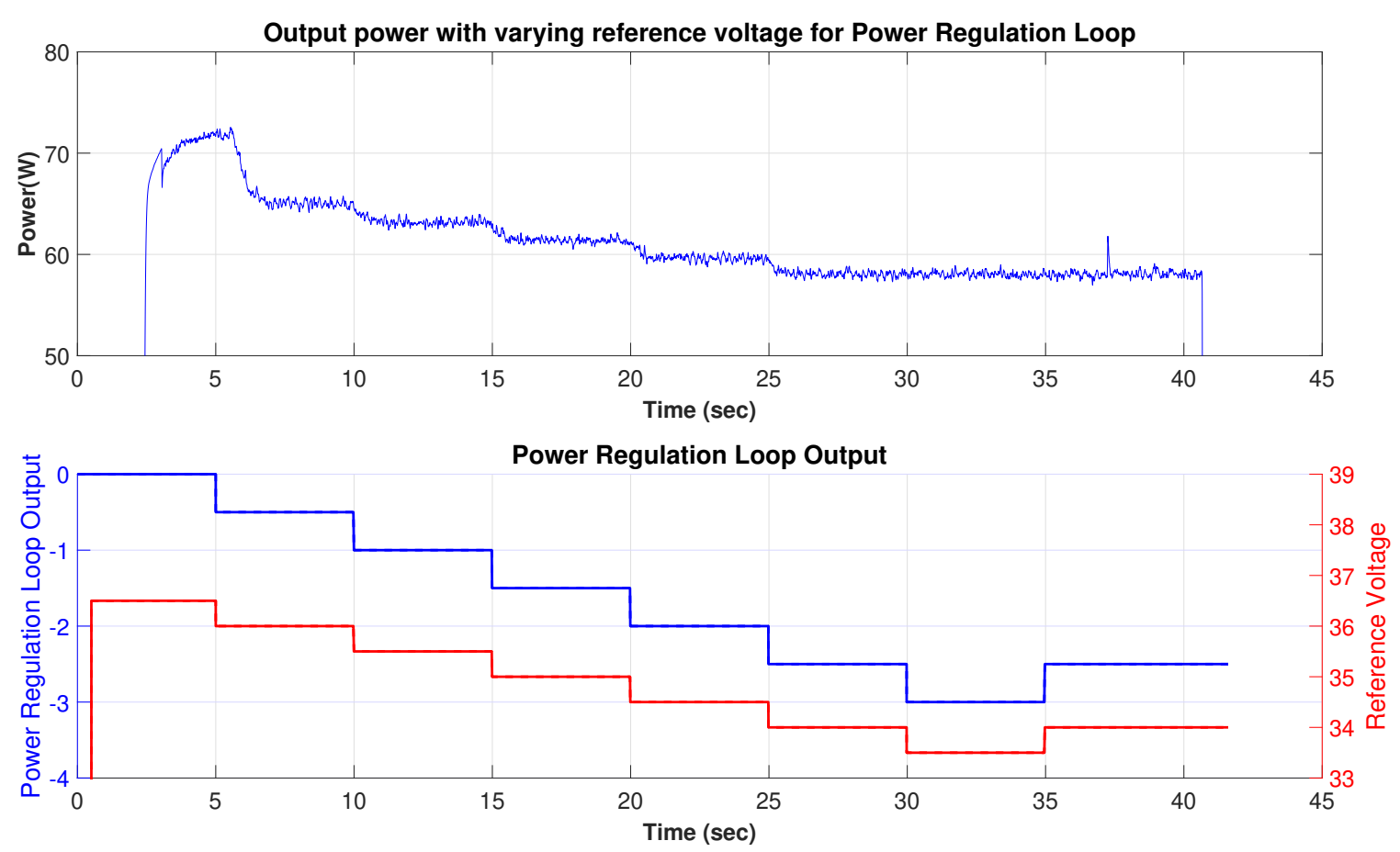

Figure 5.17: Plots of RSVC laboratory prototype with voltage regulation loop test results.

This result holds significant importance becasue the load power is dynamically changed such that the load consumes the least power within the permissible voltage levels. The effectiveness of the closed-loop RSVC (power and voltage regulation) shows that this device can dynamically adjust to the power requirements for the loads. Therefore, the closed-loop RSVC not only supports the input (source) voltage in case of an unstable power supply, but also shapes the load demand such that the minimum power is consumed. This fact is of great importance for the electric utilities when CVR is implemented. The autonomous operation of this smart device will maximize the benefits of CVR and peak shaping. Since the two regulation loops are decoupled, it allows the two loops to function independently of each other. When the power regulation loop is not required, it can be disconnected from the control system, i.e., during the normal operation. Similarly, when the voltage regulation loop is not required, the RSVC can be operated such that the net 
reactive power provided by the RSVC is 0 , as shown in the simulation results in Chapter 4 . The second plot in Figure 5.17 shows the output for the power regulation loop as well as its effect on the reference voltage.

The RSVC is practically tested in both capacitive and inductive modes to boost or suppress the output voltage. Moreover, it is shown that the RSVC can regulate the power for loads such that the loads utilize the minimum power. The RSVC can be integrated into different kinds of electrical loads, such as water heaters and lighting systems to create loads that are adaptive to the electrical grid. If many loads are equipped with the RSVC and distributed over the distribution system, these RSVC devices can provide a reliable and efficient solution for the variable power provided DERs, peak-shaving during peak demand hours, and can contribute to the delay in needed assets upgrades such as substation transformers. Moreover, the solution based on the RSVC technology can work autonomously without any (or minimum) Information and Communication Technology (ICT) built into it. 


\section{CHAPTER 6}

\section{RESIDENTIAL PEAK POWER DEMAND SHAVING BY SINGLE-PHASE RESIDENTIAL STATIC VAR COMPENSATORS}

The purpose of peak-shaving is to reduce the peak power demand supplied by electric utility companies during peak hours of operation. Utility companies usually implement time-ofuse (TOU) tariffs based on demand, where pricing is usually the highest during periods of high electrical demand [69]. The pricing structure allows utilities to promote the use of electricity during off-peak hours, thus, avoiding the need to add new generation capacity to meet the peak power demand. However, due to increased peak load, this additional generation capacity becomes an undue monetary burden to electric utilities. Instead of installing new generation capacity, electric utilities can implement solutions that can reduce power consumption during peak-demand hours. Such solutions not only help to defer the cost of additional electricity generation during peak-demand hours but also delay the asset's up-grade costs such as the cost for upgrading transformers or installing new generation.

Peak-demand shaving using battery-based solutions are popular [70-72]. Batteries are of particular interest in small and medium scales due to advanced power storage technologies, higher energy densities, low noise levels, and low maintenance requirements. Reduced costs to manufacture lithium-ion batteries for electric vehicles and portable electronics have made battery systems more affordable, and it is widely expected that prices will drop in the future. However, such a solution requires batteries to be charged during 
off-peak hours. With high penetration of renewables in the distribution system, it is difficult to predict the energy generation from renewables at any given instant of time. Hence, battery energy storage systems (BESS) have operational limitations that can render them useless during peak-demand hours.

Presently, BESS has a typical round trip efficiency in the order of $80 \%$, increasing the energy needs of the system to compensate for the losses on the BESS. Moreover, lithium-ion technology is sensitive to operating temperatures and requires installation of climate control devices, thus increasing operational and maintenance costs and requirements. Peak demand-side management using load-shedding or using appliance scheduling is another possible solution [73]. However, demand-side management using appliance's load-shedding can only be done to a certain extent before it becomes a comfort issue for residential customers. Demand-side management requires customer participation, thus limiting the ability of electric utilities to perform peak-demand in a specific area.

The objective of this work is to propose a solution for residential peak power demand shaving by using single-phase Residential Static VAR Compensators (RSVCs). Threephase Static VAR Compensators (SVCs) are used at the transmission level to regulate voltages, but they are not implemented as single-phase devices at the residential level. An RSVC is a controllable reactive power generator or absorber. The capacitive or inductive current at the output of an RSVC is adjusted to maintain a certain voltage range at the point of interconnection. Traditional SVCs employ thyristor-based switches to control their reactive power output. However, with an advancement in the development of power transistors, devices such as power MOSFETs and IGBTs, RSVC can be constructed using modern power electronic devices as discussed in earlier chapters.

A laboratory prototype of a single-phase Residential Static VAR Compensator using a fixed-capacitor with a PWM-based switched reactor has been shown to work successfully 
for regulating voltage and power. Instead of using a single bi-directional thyristor-valve to control the reactive power supplied by the reactor, PWM-based SVC uses two bi-directional switches. The additional switch acts as a by-pass switch for inductor current when the reactor is not connected to the regulation bus. The principal advantage of a PWM-based RSVC is the ability to move the low-order harmonics created by a single-phase thyristorcontrolled reactor in SVC into the PWM frequency band. An RSVC can provide dynamic reactive power, $Q_{R S V C}$, at the point of connection to the power system. The output reactive power of the RSVC is the difference between the reactive power from the PWM-based switched reactor, $Q_{L}(D)$, and the fixed capacitor, $Q_{C}$, which is expressed as,

$$
Q_{R S V C}=Q_{C}-Q_{L}(D)=V_{s}^{2}\left(\frac{1}{X_{C}}-\frac{D^{2}}{X_{L}}\right)
$$

where $V_{s}$ is the source voltage and $D$ is the duty cycle for the SW1. This novel usage of the bi-directional switching topology allows a three-phase transmission-level SVC to be used on a single-phase distribution circuit: in actuality, as a Residential Static VAR Compensator (RSVC).

The chapter briefly describes the methodology for peak-shaving on the IEEE-13 node test feeder. A description of an actual feeder, used of the study, that is located in the northwest of the United States is also given. Moreover, a detailed approach for peakshaving by using traditional methods and by using RSVC devices on the feeder is also presented in this chapter. 


\subsection{Peak Shaving on IEEE-13 Node Feeder using RSVCs}

The IEEE-13 node test feeder ${ }^{1}$ is used as a model to implement peak-shaving during peak-demand hours. The IEEE-13 node feeder is a radial distribution feeder and its singleline diagram is shown in Fig. 6.1. This feeder is included in the OpenDSS software package. The simulations were performed using MATLAB to control OpenDSS through the Windows COM interface, with GridPV Toolbox used for data extraction and plot generation.

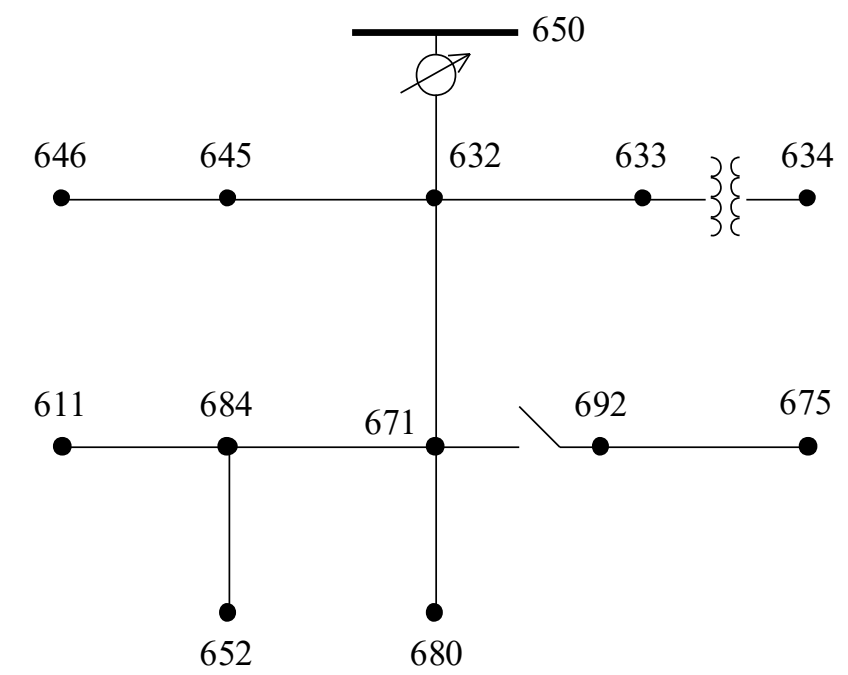

Figure 6.1: Single-line diagram for the IEEE-13 node test feeder.

In a traditional approach to peak-shaving, electric utilities lower the voltage at the feeder source as well as at the in-line voltage regulators. This approach is commonly known as Conservation of power by Voltage Reduction (CVR). This conventional approach of reducing power consumption is relevant as long as the minimum residential voltage stays above $0.95 \mathrm{pu}$ (114 V in $120 \mathrm{~V}$ base). Such an approach will reduce peak power demand;

\footnotetext{
${ }^{1}$ The loads in the feeder were modeled as constant impedance loads.
} 
however, the percentage of power reduction might be minimal because of a large difference in the voltage levels of the buses in the distribution system.

For the IEEE-13 node feeder, the minimum voltage across the feeder is $0.966 \mathrm{pu}$. The voltage profile for the distribution feeder is shown in Fig. 6.2. When the voltage at the feeder source bus is lowered, the customers will experience an undervoltage violation. Therefore, for such a distribution feeder, peak-demand shaving becomes a challenge. In the simulation, the peak power demand for the distribution circuit was 3.53 MW.

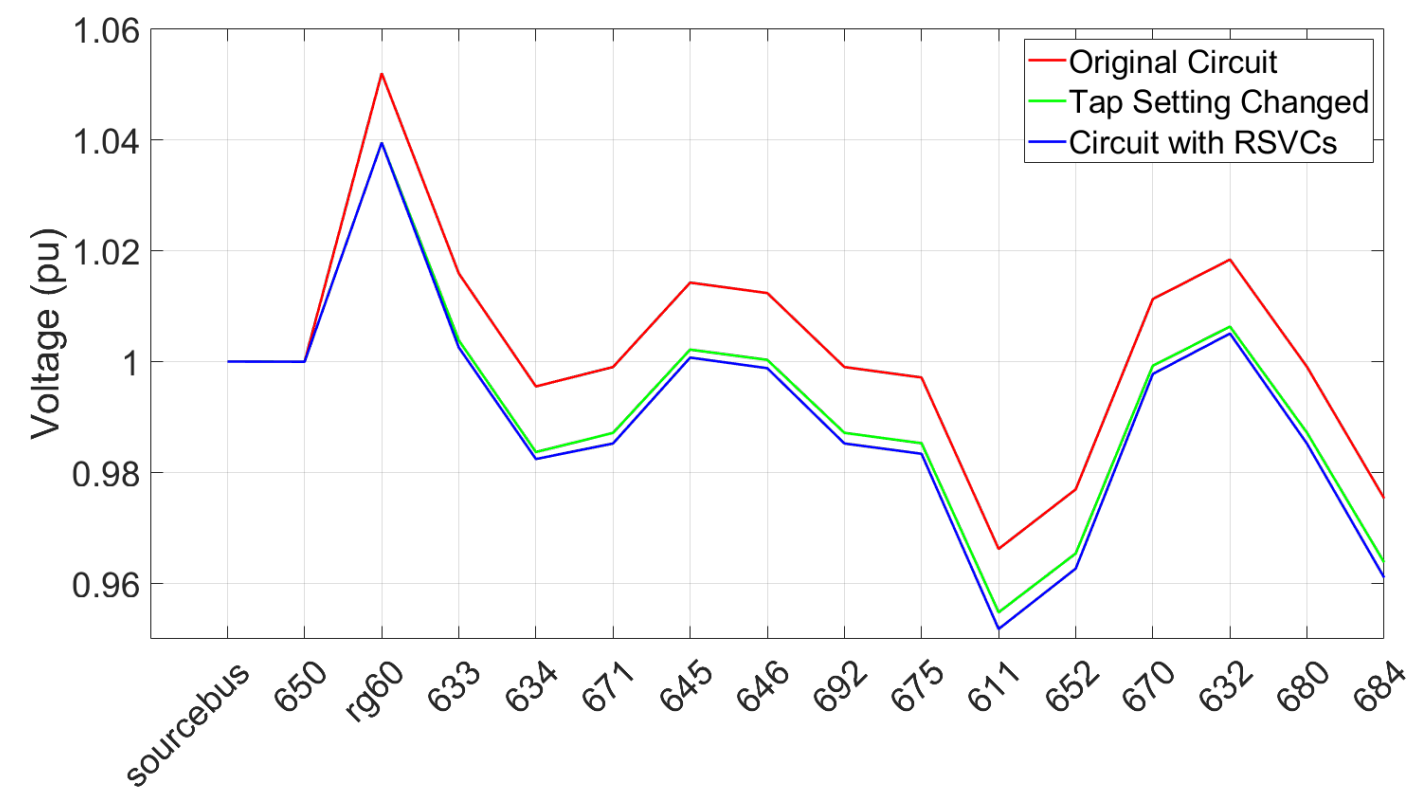

Bus Name

Figure 6.2: Voltage profile for the IEEE-13 node feeder with and without RSVCs.

By using the RSVCs, peak-shaving can be achieved efficiently. In OpenDSS, the RSVC devices were modeled as generators with the capacitive and reactive powers set to the prototype developed at Boise State University. The nameplate capacity of a single-phase RSVC was 10-kVAR capacitive and 15-kVAR inductive. The effect of RSVCs to the IEEE-13 node feeders was studied by deploying an RSVC to each bus except buses 611, 692 and 675. Buses 611 and 675 have single and three-phase fixed-capacitors of 100 and 
600 kVARs respectively. Moreover, the switch, as shown in Figure 6.1, is normally open, thus isolating the buses 692 and 675 from the rest of the distribution feeder. A power flow simulation was performed with the updated circuit. Because the RSVC can operate in both capacitive and inductive modes, the undervoltage violations can easily be avoided for the loads experiencing an undervoltage. Figure 6.2 also shows the voltage for the distribution feeder when the feeder source bus is lowered to 1.0 with and without RSVCs regulating the voltage at each bus. From the voltage profile, the voltage of the distribution with the RSVC devices is lower than the voltage profile of the feeder without RSVC. This is because the RSVCs are operating in the inductive mode to regulate the voltage. Using the RSVC devices, the system operators can be confident in lowering the voltage at the feeder source so that the voltage at the customers' locations will not violate the ANSI standard C84.1. Figure 6.3 shows the peak power demand by adding an RSVC to the individual busses on the IEEE-13 node feeder. The peak power demand was reduced from 3.53 MW to 3.43 MW by adding nine RSVC devices across the distribution feeder.

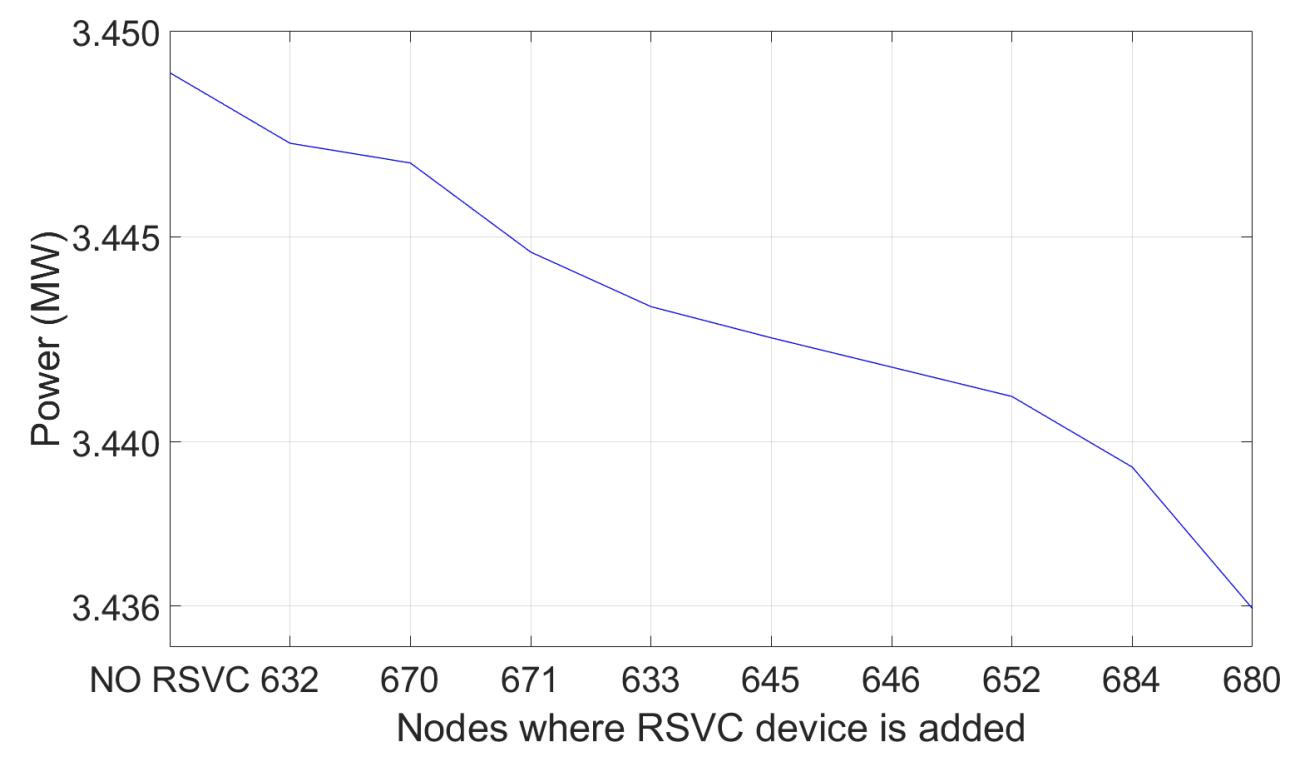

Figure 6.3: Peak power demand as function of the number of RSVCs. 


\subsection{Peak Shaving on an Actual Feeder using RSVCs}

Initial results from the IEEE 13 -node test feeder show that a $2.83 \%$ reduction in peakdemand can be achieved by using single-phase RSVCs. However, in the previous case RSVC devices were connected at each bus of the distribution feeder. This section involves a study to perform peak-shaving for a feeder in the northwest part of the United States by deploying the RSVC devices using the Worst-offender algorithm. This approach will identify the busses (or loads) that hinder implementing CVR throughout the distribution feeder. Adding RSVC devices to those busses will ensure the participation of the entire feeder for peak-shaving using CVR and maximize the benefits for electric utilities associated with peak-demand reduction.

\subsubsection{Distribution Feeder Description}

The feeder selected for the study has a pronounced peak load of $2.62 \mathrm{MW}$ that occurs only for a few days during the year. Therefore, peak-shaving can be used to reduce the peak-demand of the feeder and defer the need to upgrade any assets within the feeder, especially the substation transformer. The feeder operates at $12 \mathrm{kV}$ line-to-neutral voltage and at a length of 16 feeder miles. The loads are modeled as spot loads connected directly to the feeder (i.e. no service transformers are modeled). The distribution feeder serves a total of 471 residential, commercial, and industrial customers. Figure 6.4 shows the topology of the test feeder. The voltage regulation of the feeder is performed by an LTC transformer located at the feeder source as well as four in-line voltage regulators. The feeder also

contains a fixed capacitor bank that was installed for power factor correction. Figure 6.4 also shows the per-unit voltage of each bus under peak load conditions. The original tap settings of the LTC transformer and in-line regulators are given in Table 6.1. 


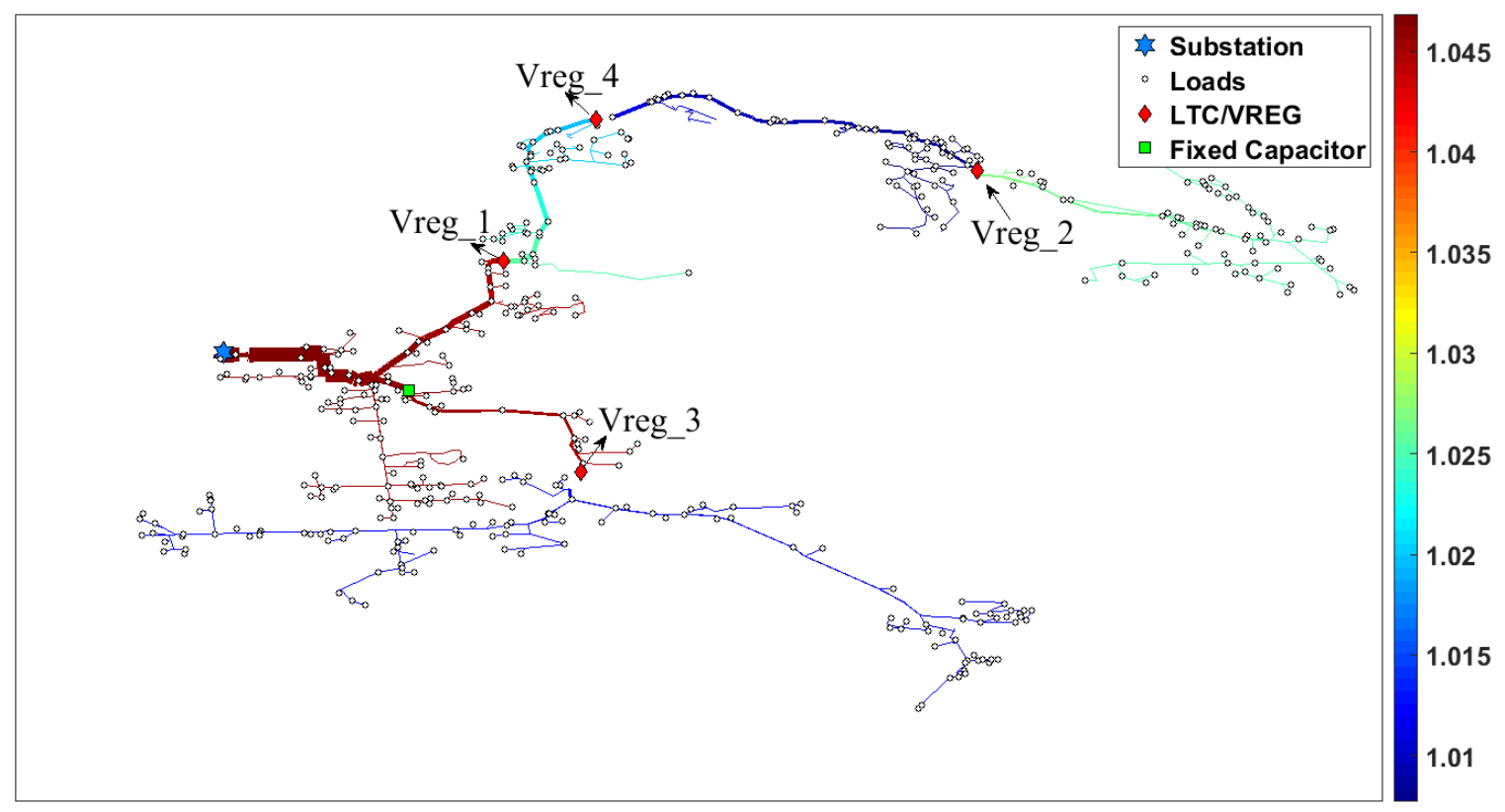

Figure 6.4: Feeder lines and voltage (pu) for the distribution feeder.

Table 6.1: LTC and voltage regulator settings in the feeder without CVR.

\begin{tabular}{|c|c|}
\hline Voltage Regulators & Voltage Level (V/pu) \\
\hline \hline Transformer (LTC at substation head ) & $123 / 1.025$ \\
\hline Voltage Regulator (Vreg_1) & $123 / 1.025$ \\
\hline Voltage Regulator (Vreg_2) & $123 / 1.025$ \\
\hline Voltage Regulator (Vreg_3) & $121 / 1.008$ \\
\hline Voltage Regulator (Vreg_4) & $121 / 1.008$ \\
\hline
\end{tabular}

\subsubsection{Load Model}

Distribution systems supply power to a wide range of loads, ranging from rural areas to urban areas. The loads can generally be grouped into three categories depending on how their power demand varies with respect to the voltage at their terminals: constant Power(P) load, constant current (I) load and constant impedance (Z) load. For constant power (P) load, the power demand remains constant regardless of the voltage at the terminals of the 
load. For constant current (I) load, the power demand is proportional to the load voltage. For the constant impedance $(\mathrm{Z})$ load, the power demand is proportional to the terminal voltage squared. Distribution feeders mainly contain constant impedance (Z) and constant power (P) loads, with few constant current loads. For this study, the distribution feeder was assumed to contain $40 \%$ constant power load and $60 \%$ constant impedance load [74].

\subsection{Power and Voltage Profile for the Distribution Feeder}

Figure 6.5 shows the annual power demand profile for the distribution feeder for 2016. The 3-phase power was measured at the feeder source by adding an OpenDSS monitor on each phase at the transformer LTC. The simulation was performed for 8760 points (365 days) in one-hour intervals. The total power supplied by the substation is calculated by adding the power of each phase for every hour. The envelope of the Fig. 6.5, plotted in red, shows the daily peak-demand throughout the year. It can be seen that the distribution feeder experiences a peak-demand during the winter season, i.e., from November till February. In other words, the distribution circuit has a winter peak power demand. To satisfy the rise in power demand during the winter season, the electric utility normally activates the operating reserves to meet the rising peak-demand.

The peak-demand in the area can be decreased by reducing the voltage levels across the distribution feeder. However, to perform peak-shaving by CVR, the electric utility needs to consider the effect of lowering the voltage at the feeder source. In the case when the voltage at the feeder source is lowered too much, the voltage delivered to the customers may fall below the ANSI C84.1 standard. Therefore, the electric utilities seek other solutions before lowering the voltage at the substation.

For the first set of simulations, the voltage and power profiles across the feeder were 


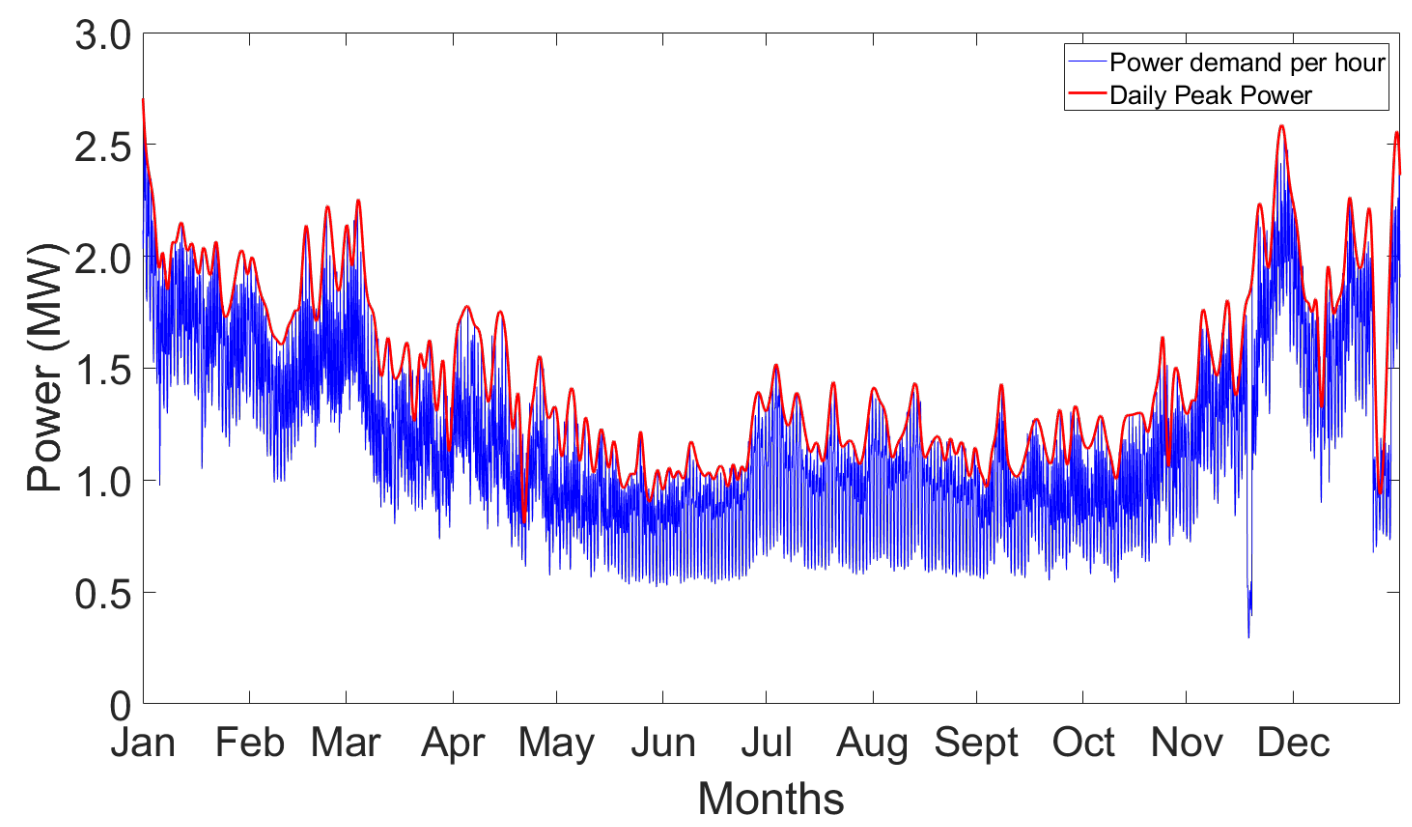

Figure 6.5: Annual power demand profile for the distribution feeder.

plotted with the existing settings, given in Table 6.1, for the substation LTC and in-line voltage regulators. The annual power profile is shown in Fig. 6.5 while the annual voltage profile is shown in Fig. 6.6, plotted with a 1-hour time interval. The envelope of Fig. 6.5 shows the daily peak power demand whereas the envelopes of Fig. 6.6 show the daily maximum and minimum voltages across the distribution feeder.

For this study, only peak power demand during the winter season is considered. Figure 6.7 shows the daily winter power profile for the distribution feeder from November to February. The peak power demand during the winter season is $2.62 \mathrm{MW}$. The highlighted areas in the figure show the peak power days during the winter season. 


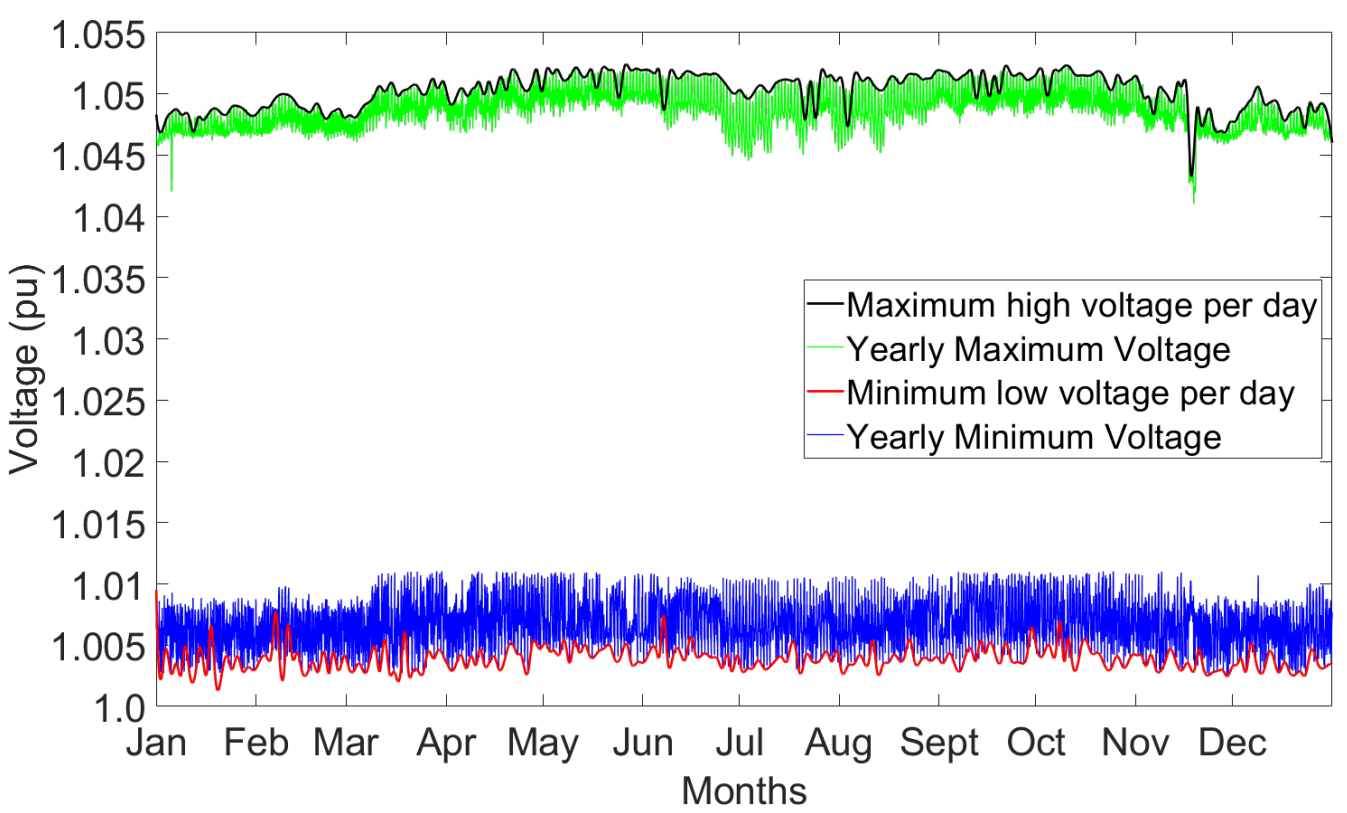

Figure 6.6: Yearly voltage profile for the distribution feeder.

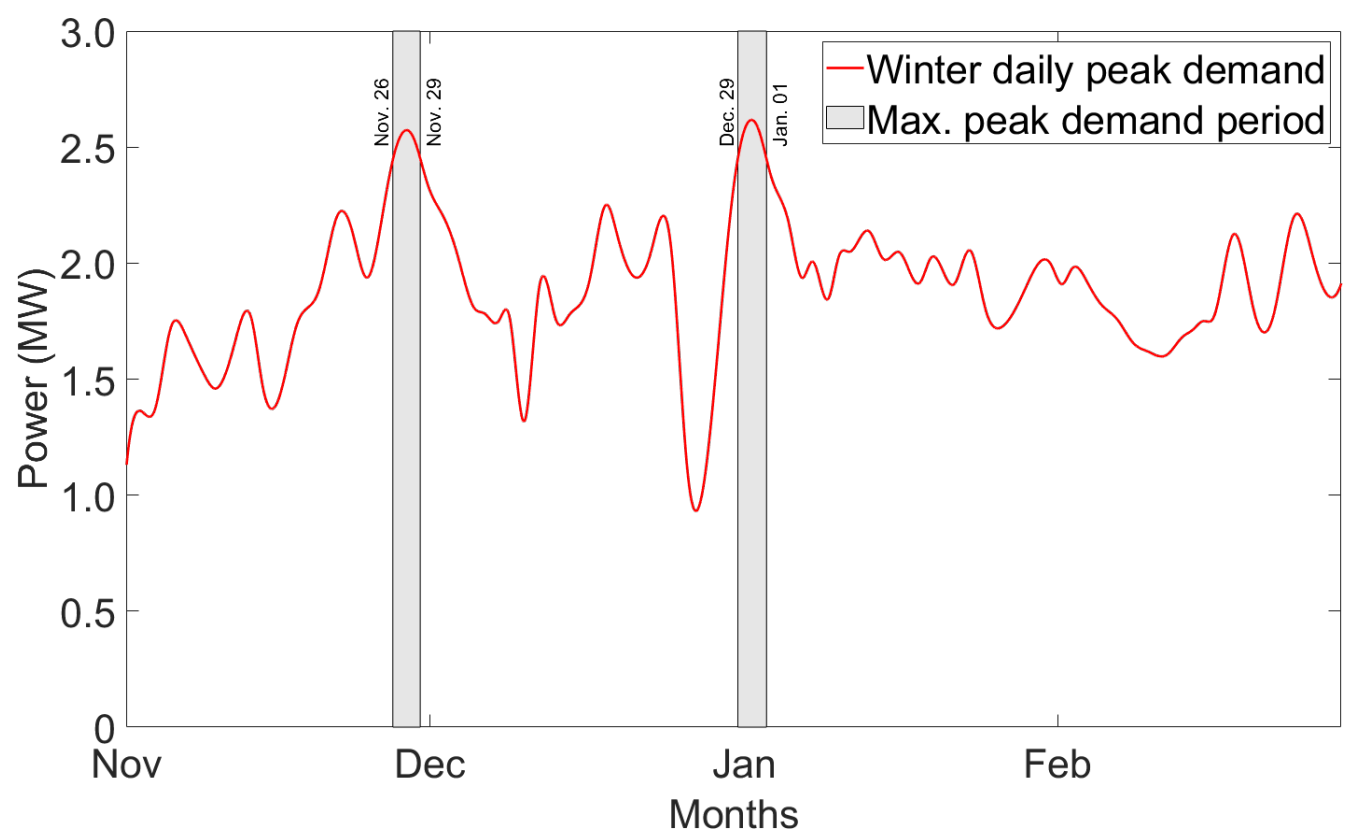

Figure 6.7: Winter power demand profile for the distribution feeder. 


\subsection{A General Way of Approaching Peak Shaving Using CVR}

A traditional way to approach peak-shaving using CVR is to change the settings on the LTC transformer and the in-line voltage regulators. As mentioned earlier, the problem associated with such an approach is that the electric utilities must have adequate flexibility to address the variability and uncertainty in power demand. If the power demand increases more than the anticipated power requirements, a lower tap setting will result in the voltage being lower than the limit set by the ANSI C84.1 standard. Although tap controllers can switch the taps for the LTC and voltage regulators fairly quickly (generally within few seconds to a minute), electrical distribution planners always keep a margin of flexibility to cater to the changing nature of the loads. The size of that margin is based on the lowest voltage level on the distribution feeder. Therefore, electrical utilities cannot fully benefit from peak-shaving during peak-demand hours.

The maximum benefit from CVR can be obtained when the voltage across the entire distribution feeder is ideally at its lowest limit $(0.95 \mathrm{pu})$. This fact will ensure maximum savings from peak-shaving as well as help distribution planners delay the requirements for additional capacity to meet the anticipated power demand. The discussion in this section focuses on the traditional method for approaching CVR by lowering the tap setting at the substation LTC transformer as well as the in-line voltage regulators in the distribution feeder.

\subsubsection{Changing the Substation LTC and Voltage Regulator Tap Settings}

Figure 6.8 shows the voltage levels of the distribution feeder at different settings of the substation LTC and four in-line voltage regulators plotted using daily minimum and maximum voltage levels. Three sets of simulations were performed after changing the devices band 
center settings to $118 \mathrm{~V}, 117 \mathrm{~V}$, and $116 \mathrm{~V}$. The plots in Fig. 6.8 show the daily minimum and maximum voltage levels across the distribution feeder. It can be seen that when the band center is at $116 \mathrm{~V}$, the minimum voltage on the distribution feeder is less than 0.95 per-unit (pu). The plots in the Fig. 6.8 are a mere depiction of the problems and uncertainty the distribution planner faces while selecting tap settings for CVR operation.

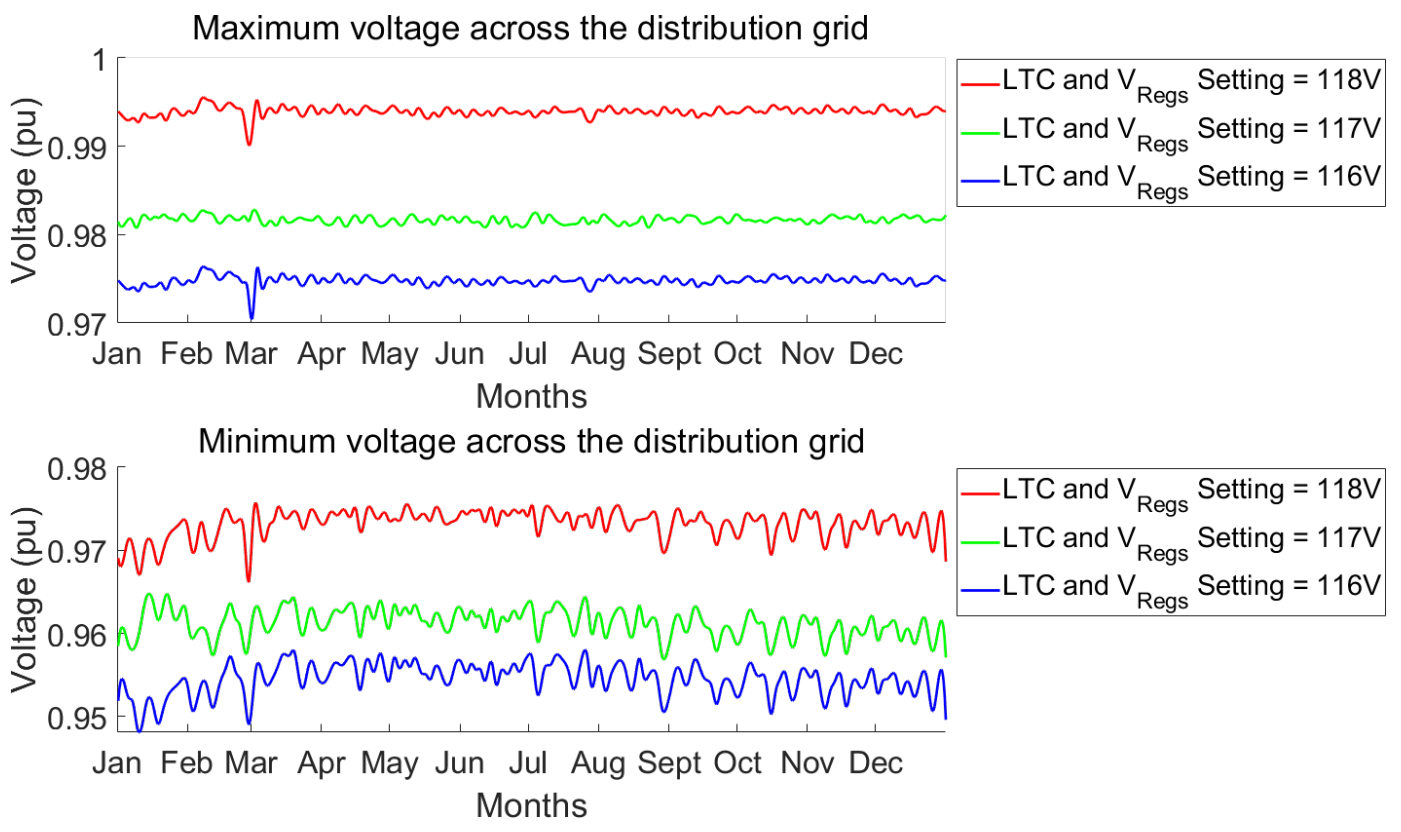

Figure 6.8: Maximum and minimum voltage across the distribution feeder at different tap settings for the substation LTC and in-line voltage regulator.

Another observation that can be drawn from the plots in Fig. 6.8 is that there is a significant difference between the minimum and maximum voltage levels of the distribution feeder. For example, when the band center is equal to $118 \mathrm{~V}$, the average maximum voltage across the feeder is $0.994 \mathrm{pu}$ and the average minimum voltage across the feeder is $0.973 \mathrm{pu}$. The difference between the voltage levels across the distribution feeder prevents attaining the maximum advantage possible by using CVR. Figure 6.9 shows the winter power profile for the distribution feeder with different settings at the substation LTC and in-line voltage 
regulators. The peak power when band centers are adjusted from $123 \mathrm{~V}$ and $121 \mathrm{~V}$ to 118 $\mathrm{V}, 117 \mathrm{~V}$, and $116 \mathrm{~V}$, for the substation LTC transformer and the in-line voltage regulator, is 2.53 MW, 2.51 MW, and 2.49 MW respectively. In terms of percentage of peak power reduction compared with the original settings, the peak power is reduced by $3.43 \%, 4.2 \%$, and $4.96 \%$ when the band centers are equal to $118 \mathrm{~V}, 117 \mathrm{~V}$, and $116 \mathrm{~V}$ respectively.

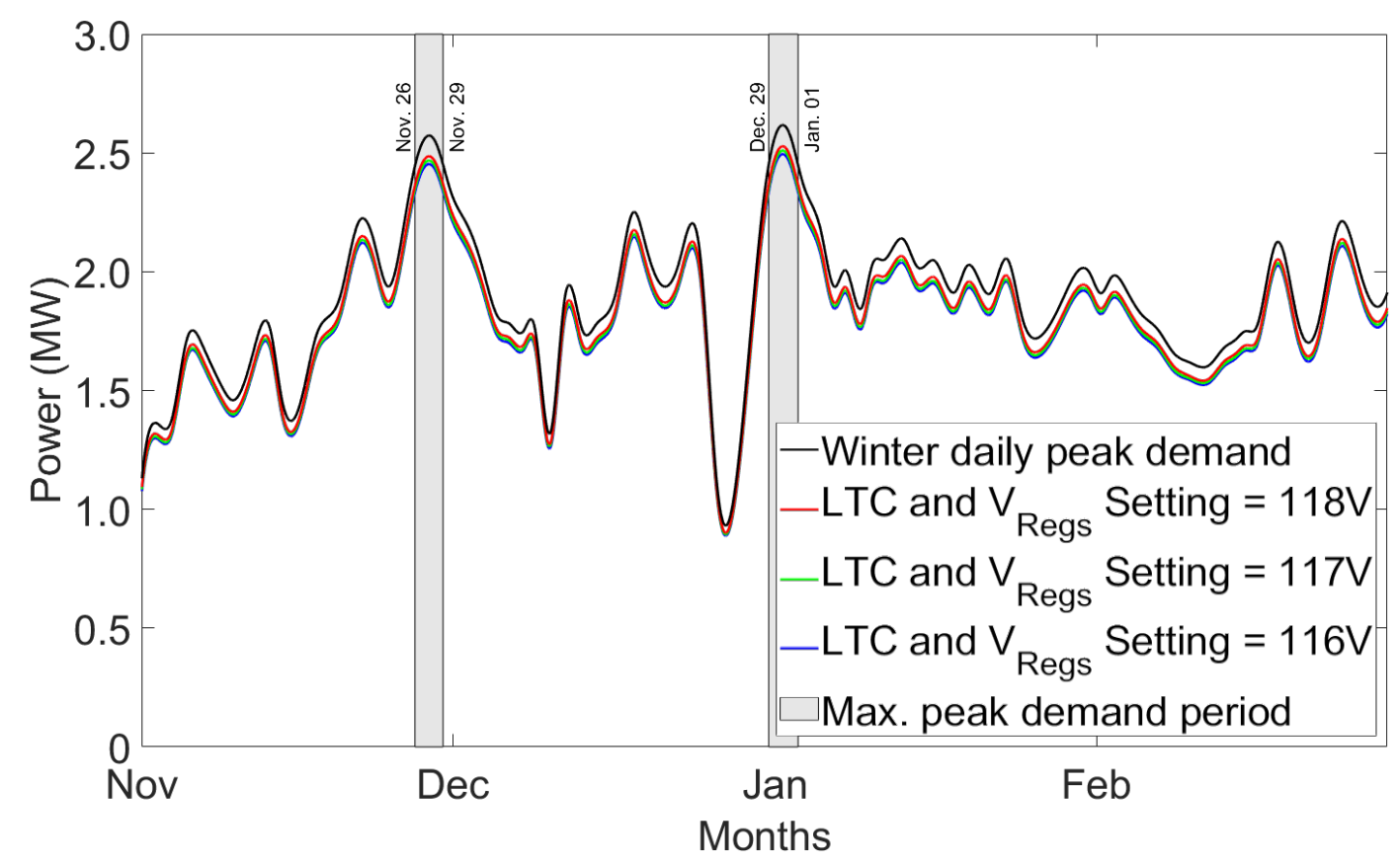

Figure 6.9: Winter power demand profile for the distribution feeder at different tap settings.

Although the peak power reduction is significant by changing the band centers for the LTC and in-line voltage regulators, the lack of concrete evidence regarding the minimum voltage at the distribution circuit can lead the system operators to operate at higher voltage levels. Forecasting and planning help ensure utilities have a fairly accurate load profile but with the integration of renewable resources on the distribution system, these forecasts are prone to more errors. At the time of writing this paper, there is no commercial device that can perform voltage regulation independently, in inductive and capacitive modes, at 
multiple points (locally) on the distribution feeder.

\subsection{Peak Shaving Using Single-Phase RSVC}

The use of RSVC devices could enhance the implementation of CVR by raising the voltage in areas that experience low voltage when the LTC settings are lowered and lowering the voltage in areas that operate at a higher voltage. The limitations for RSVC lie in the sizing of the RSVC components (fixed-capacitor and switched inductor). However when RSVC components can be scaled, based on the requirements at a specific location, RSVC can be a suitable alternative for peak-shaving rather than the conventional BESS.

\subsubsection{Simulation Method}

The first step to perform peak-shaving is to analyze the power profile and determine the peak power demand season. Figure 6.7 shows the winter power profile for the distribution feeder. To perform peak-shaving using RSVC, the following steps were performed:

(i) During the first round of simulation, the voltage at the substation and at the four voltage regulators was lowered to $116 \mathrm{~V}^{2}$ An hourly power flow simulation was performed to identify the buses that have voltages below the $0.955 \mathrm{pu}$. Since the RSVC can operate in both inductive and capacitive mode, the buses that have voltages greater than $0.97 \mathrm{pu}$ were also identified.

(ii) For the RSVC deployment strategy, the RSVC devices were placed on each of the buses with a voltage level greater than $0.97 \mathrm{pu}$ and less than $0.955 \mathrm{pu}$. A power flow simulation was repeated until none of the buses had a low-voltage violation as

\footnotetext{
${ }^{2}$ Band center setting of $116 \mathrm{~V}$ was chosen because the model used does not represent the service transformer or the service drop. Maintaining $116 \mathrm{~V}$ at the primary side of the service transformer will enable the voltage to be at $114 \mathrm{~V}$ at the meter terminals.
} 
outlined in the ANSI standard. The data collected from the RSVC devices placement were used to determine how the RSVC devices placement affects the overall capability of the feeder to perform CVR.

The flowchart for the RSVC deployment is shown in Figure 6.10. The locations for the deployment of the RSVC devices across the distribution feeder is shown in Figure 6.11.

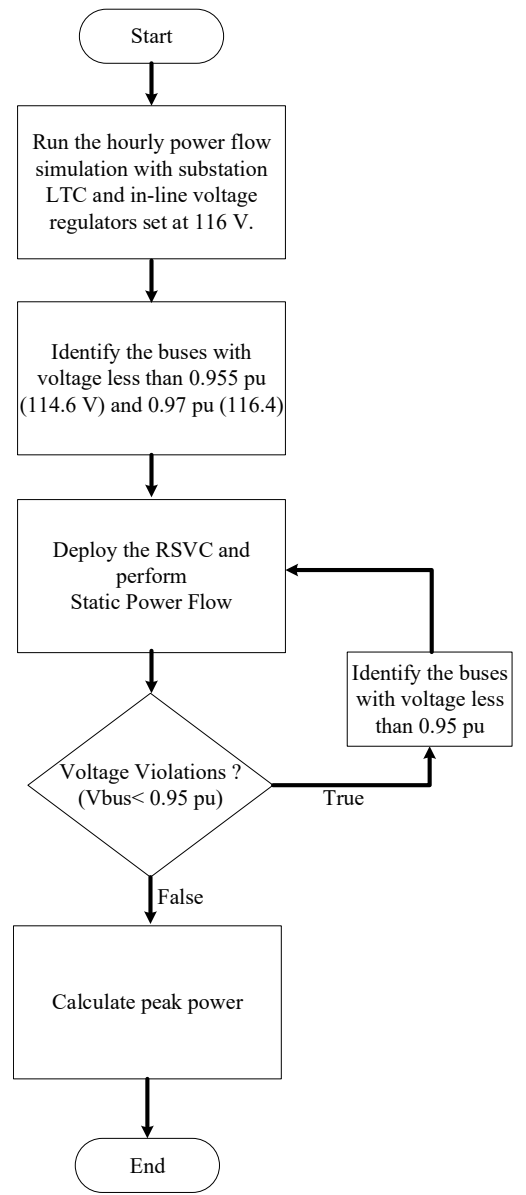

Figure 6.10: Flowchart for RSVC deployment strategy based on Worst-offender algorithm. 
Voltage (pu) and deployment locations for RSVC

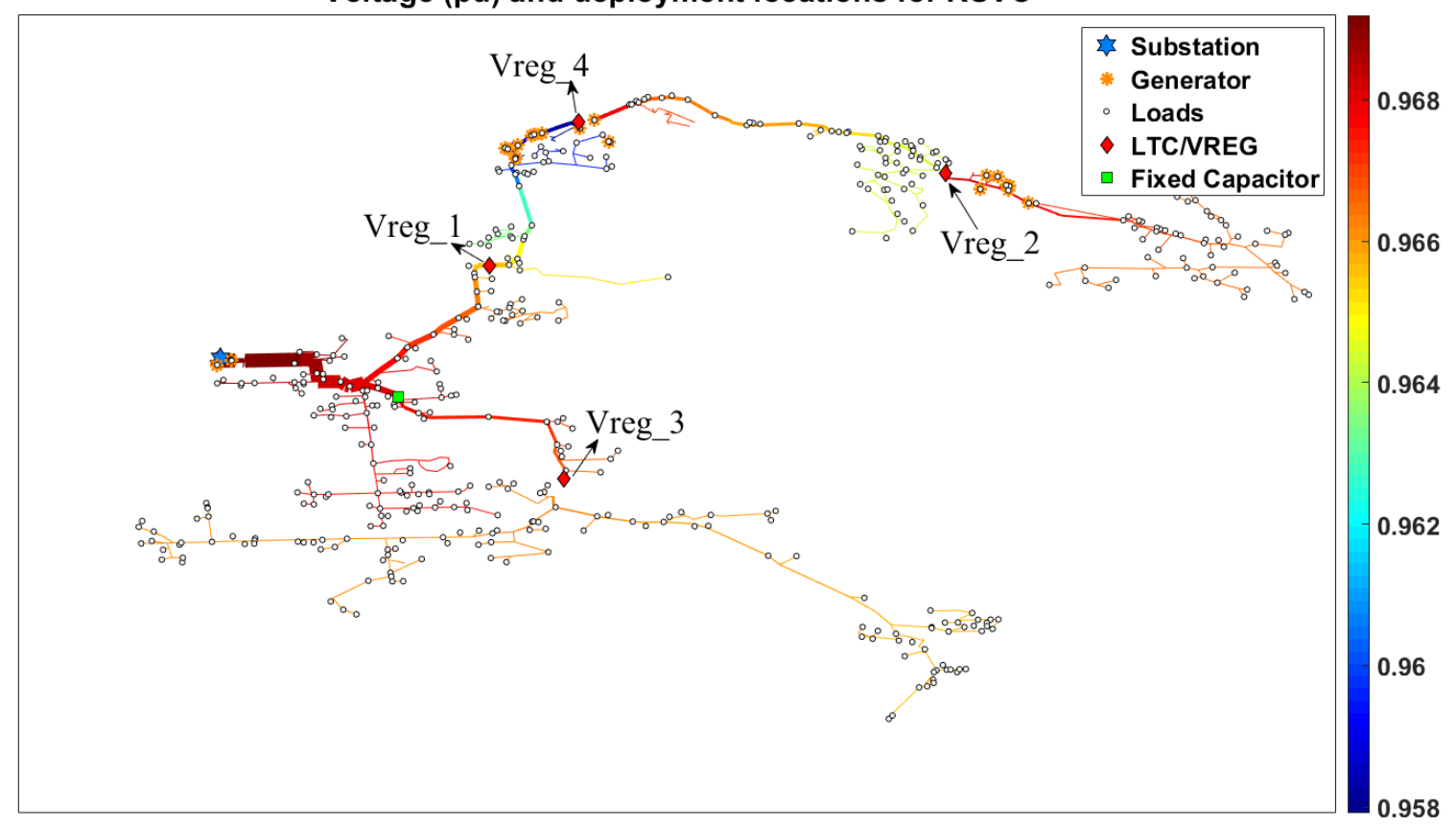

Figure 6.11: RSVC placement in the distribution circuit.

\subsubsection{Simulation Results}

The first step is to verify that the simulated substation voltage levels do not violate the minimum permissible voltage. The verification was made by plotting the voltage levels with and without the deployment of the RSVC devices when the band centers for the substation LTC and in-line voltage regulators are set to $116 \mathrm{~V}$. Figure 6.12 shows the minimum and maximum voltage levels across the distribution feeder throughout the year. It can be seen that the voltage violation which occurs due to bus voltages lower than 0.95 pu without RSVC is mitigated by deploying RSVC. For the other points of the simulated voltages, the minimum voltage is less than the voltage level without RSVC devices. This is because, at those days of the year, RSVC devices are acting in the inductive mode. Similarly, the maximum voltage levels with RSVC devices are lower than without RSVC. 


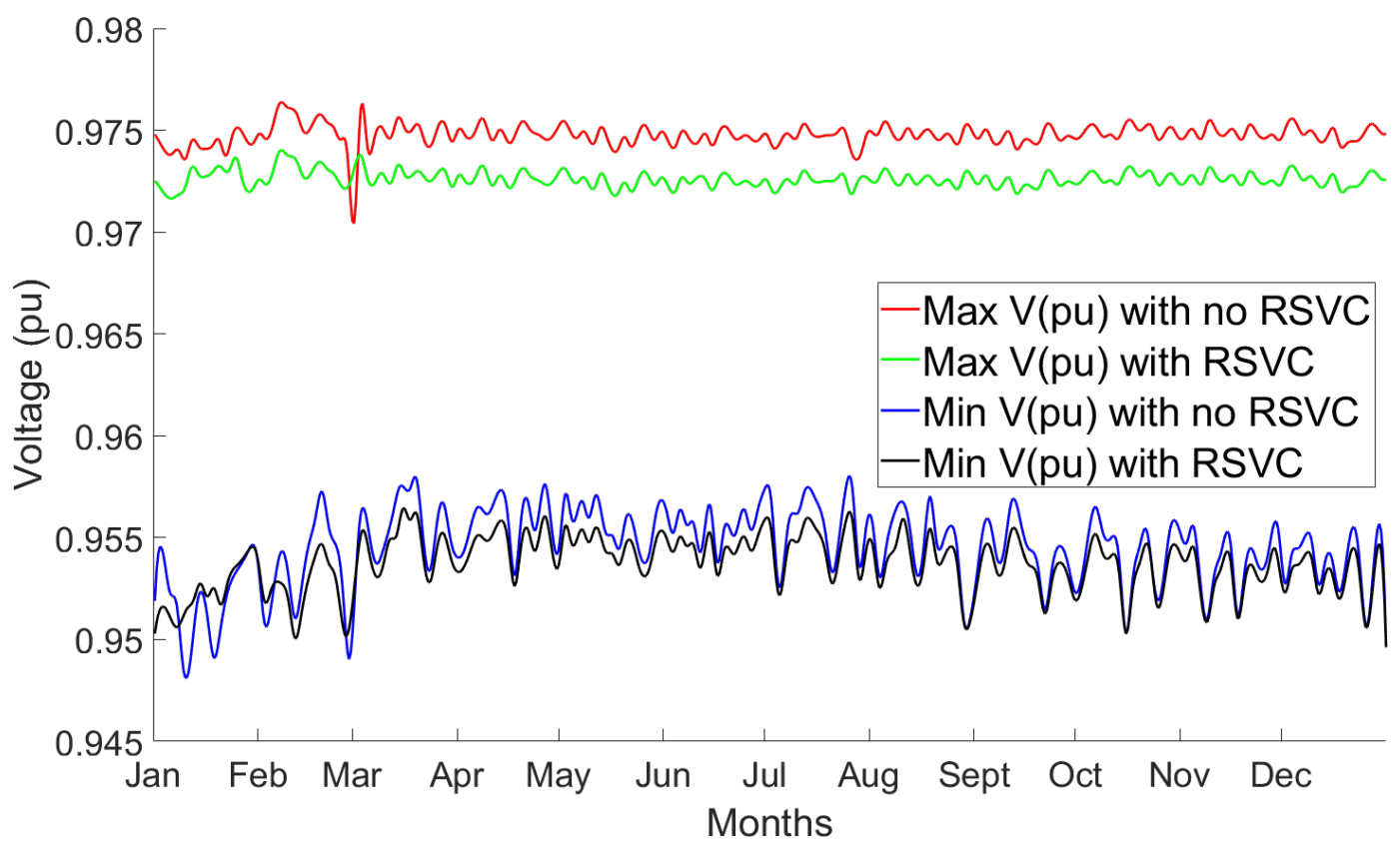

Figure 6.12: Voltage profile of the distribution with and without the RSVC when the tap settings for LTC and in-line voltage regulators is set to $116 \mathrm{~V}$.

The aim of these simulations is to show RSVC-enhanced CVR for peak-shaving. Figure 6.13 shows a comparison for winter power profiles. Three power profiles are plotted with the base case representing the power profile with the original settings as given in Table 6.1. Figure 6.13 also plots the power profile when the band center settings were changed to 118 V. Finally, the figure shows the power profile when the RSVC devices were introduced in the distribution feeder. It can be seen that the total power consumption by the distribution feeder decreases when RSVC were deployed across the circuit. The peak power in the base case, when band centers are equal to $118 \mathrm{~V}$ and when RSVC were deployed in the circuit, is $2.62 \mathrm{MW}, 2.53 \mathrm{MW}$ (3.43\% peak reduction) and $2.49 \mathrm{MW}$ (4.96\% peak reduction) respectively.

There are two ways to perform peak shaving by using CVR: (i) long-term energy reduction which will involve reducing the power peak for the entire winter season. Traditionally, 
CVR is implemented in an on-off-on again basis. Some utilities use one day on, one day off whereas others use two days on, two days off. (ii) And short-term demand reduction for the days where the peak power demand is generally higher than the overall peak. Figure 6.13 shows the long-term CVR where peak power is reduced for the entire winter season and Figure 6.14 shows the case when the peak shaving is performed for short-term. It is up the system operators which methodology is adopted to perform the peak reduction. Several factors are generally considered when taking such a decision. One of the important factors to keep in mind is the number of LTC operations and the number of in-line voltage regulator operations. If CVR is applied during the entire year, the number of operations of the LTC could increase significantly leading to more wear and tear of the LTC that results in the increased cost of maintenance and could substantially decrease the benefits for CVR.

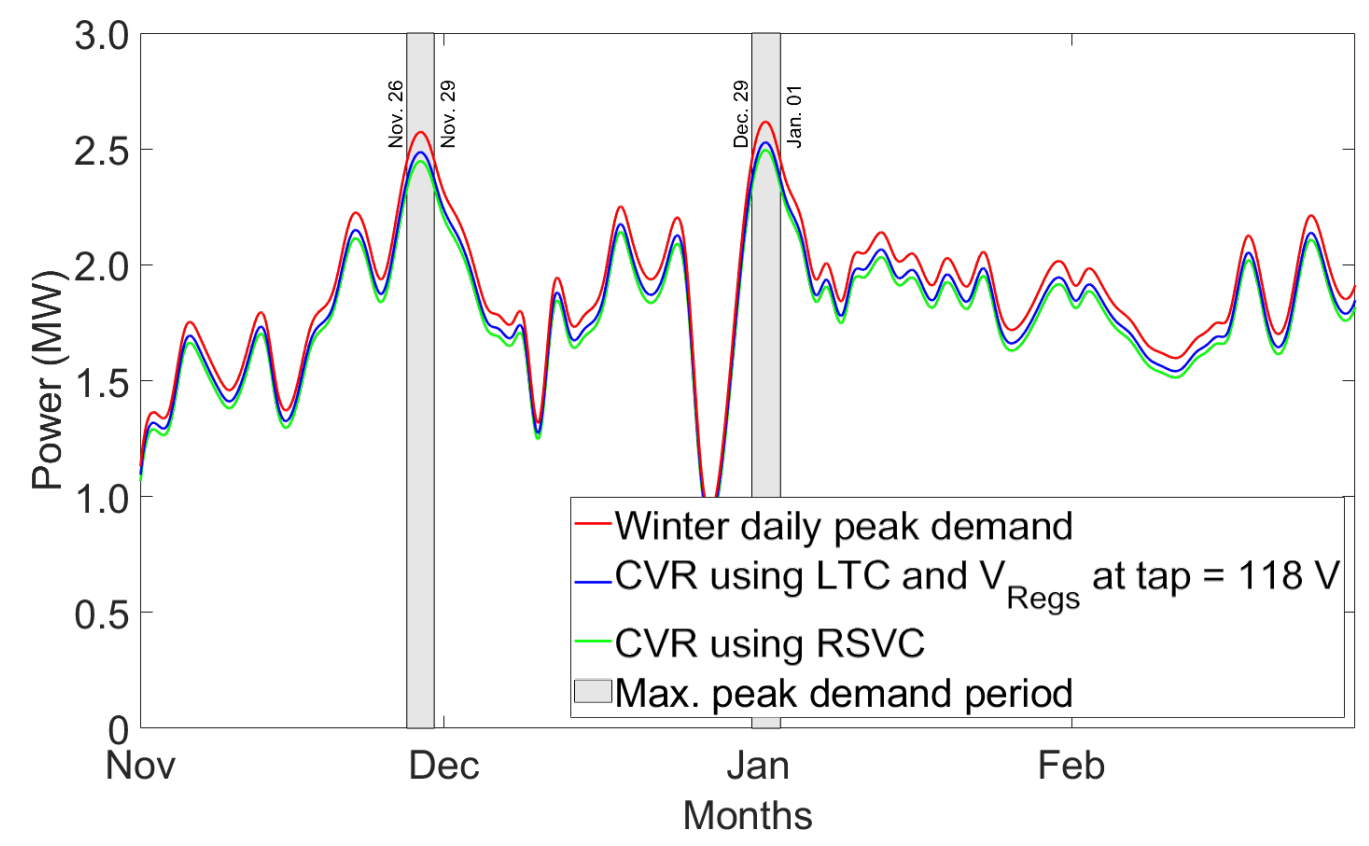

Figure 6.13: Long-term RSVC-enhanced CVR power profile for the distribution feeder.

The reactive power output of the RSVC devices for each RSVC is shown in Figure 6.15. Each point in the figure shows the net reactive power provided by the RSVC at a specific 


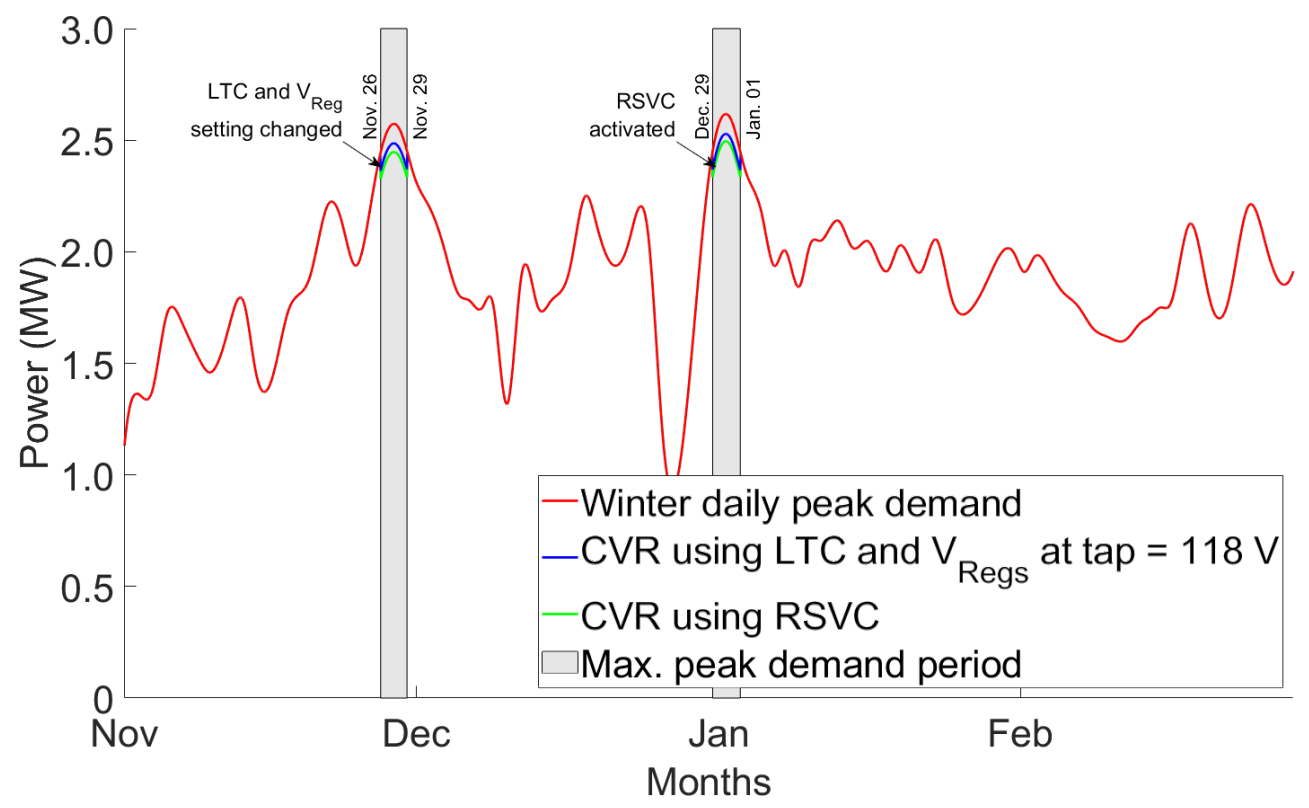

Figure 6.14: Short-term RSVC-enhanced CVR power profile for the distribution feeder.

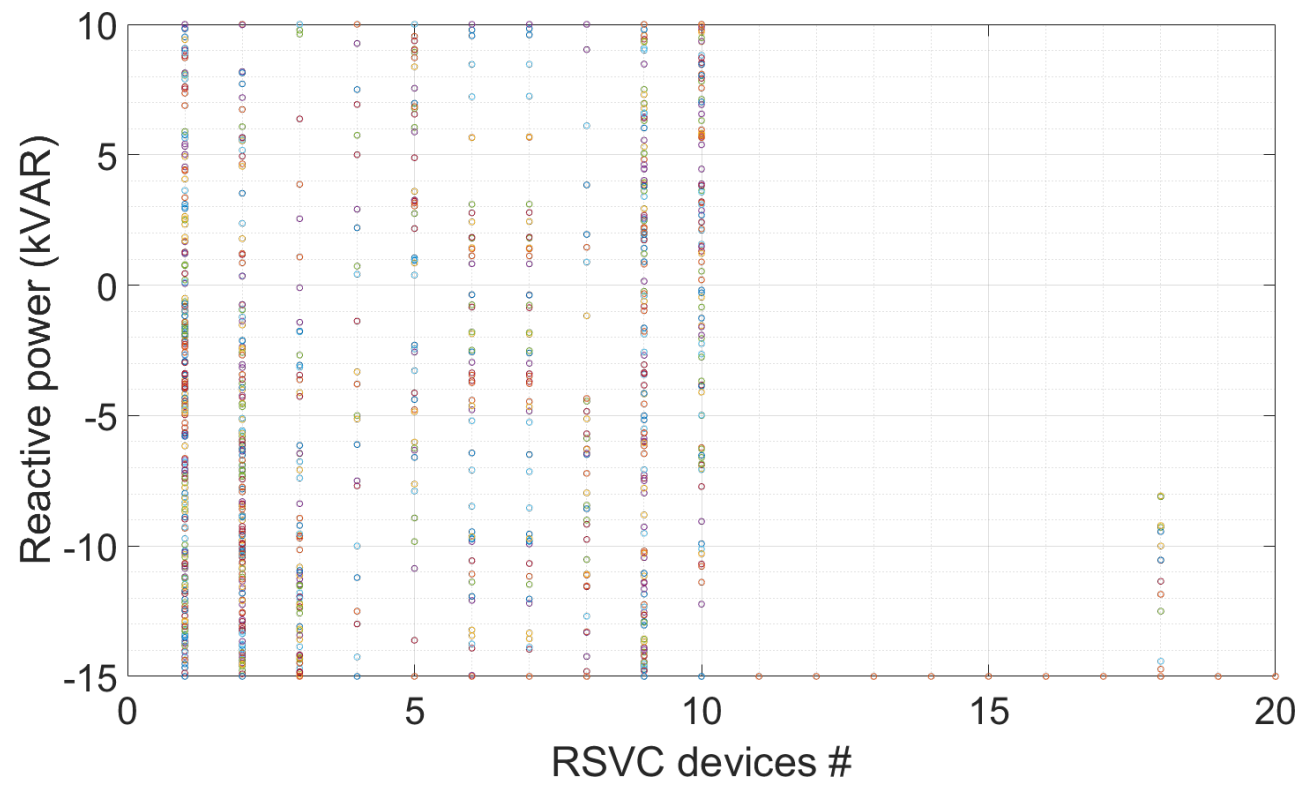

Figure 6.15: Net reactive power provided by the RSVC devices during their operation for the entire year. 
service transformer during the entire year. The result shows that RSVCs can transition from an inductive operation mode to a capacitive operating mode thus providing a better voltage regulation profile than with capacitors alone. For the sake of data representation, the reactive power in Figure 6.15, the RSVC devices numbered from 1 to 10 are the devices which are connected to busses with voltages level lower than $0.955 \mathrm{pu}$ whereas the remaining RSVC are connected to busses with voltage greater than $0.97 \mathrm{pu}$. In total, 20 RSVC devices were deployed across the distribution system.

\subsection{Cost Analysis and Performance benefits for Peak Shaving between RSVC and BESS}

Distribution planners forecast load to be able to meet peak-demand in upcoming years and to develop projects to upgrade the feeder accordingly before it is overloaded. Normally, planners seek to meet the power demand for many years in the future by accounting for the expected load growth. This process of load forecasting considers the existing capacity of the substation, anticipated plant retirements, and power supplied by distributed generation. Planning helps electrical utilities to have an adequate amount of lead time for an upgrade to the existing infrastructure.

Infrastructure deferral using RSVC-enhanced CVR was calculated for different growth rates by calculating the number of years it will take for the peak load to reach the planning capacity of the substation transformer. The planning capacity of the substation transformer is considered to be $98 \%$ of the transformer nameplate. The first calculation was performed using the peak power demand of the distribution feeder operating with settings given in Table 6.1, in other words, no CVR is performed on the distribution feeder. From the previous section, the peak power for the feeder was $2.62 \mathrm{MW}$. The substation transformer 
was assumed to be 5 MVA and, using the planning limit of $98 \%$, the capacity calculates to 4.9 MVA.

The same calculation was performed for the peak load obtained with the RSVC-enhanced CVR (2.49 MW). The years that it took to reach the planning capacity with and without RSVC-enhanced CVR is shown in Fig. 6.16. The results show that it is possible to defer the upgrade of the substation transformer, from 2 to 4 years, for a load growth ranging from $2 \%$ to $6 \% \mathrm{pu}$. The monetary value of the deferral will depend on several factors unique to each utility such as the Weighted Average Cost of Capital, standard transformer size, etc.

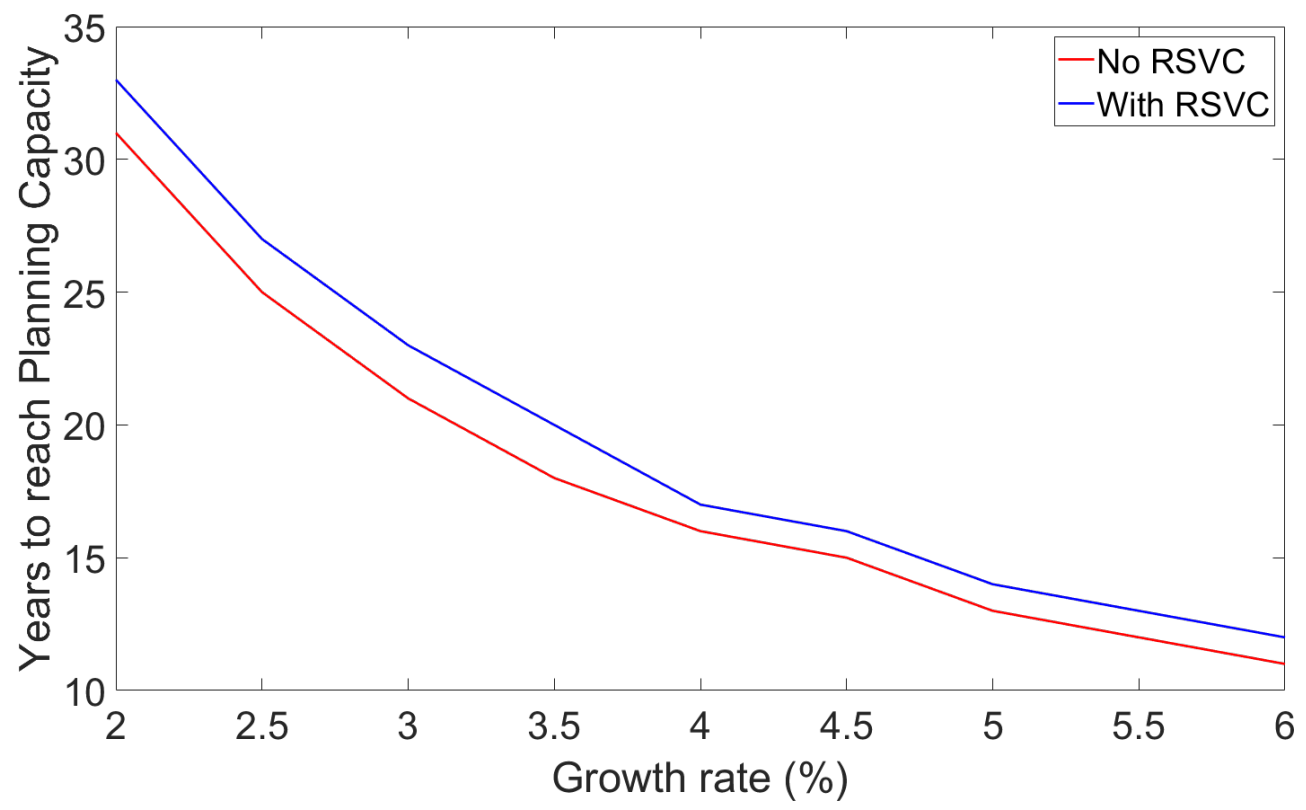

Figure 6.16: Transformer Deferral Calculation

A different strategy for peak-shaving is implemented by injecting power from the BESS. The BESS injects the power to the grid when the power demand increases a certain threshold level. This is generally referred to the discharging of the BESS. However, the peakdemand of a feeder can last for several hours, thus limiting the ability of the BESS to 
provide peak-shaving capabilities. An advantage associated with using RSVC-enhanced CVR for peak-shaving is that it can be operated continuously without any limitations.

If BESS were to be used to reduce the peak by $4.96 \%$, the capital expenditure (CapEx) will be significantly larger than the deployment of RSVC. For the given distribution feeder, the power saved as a result of peak-shaving is equal to $130 \mathrm{~kW}$. Using the recently published data for the cost associated with the storage components for BESS, the cost per $\mathrm{kW}$ is equal to $\$ 3200 / \mathrm{kW}[75]$. Therefore, the total initial CapEx will be equal to $\$ 442,000$ for using BEES to achieve the same peak-shaving. The cost mentioned above assumes a 4hour battery $(0.25 \mathrm{C})$. Therefore, the use of BESS for peak-shaving requires that the peakdemand should not last for more than 4-hours in one day. Beyond this time, the stateof-charge of the BESS will be insufficient to meet the peak-demand, and the feeder will experience the same peak load as before, thus eliminating any benefits related to peakshaving. BESS is limited in duration and requires advance forecasting, whereas the method presented in this work, RSVC-enhanced CVR, has the flexibility in its duration of the operation and lower cost. Therefore, RSVC-enhanced CVR is a more viable solution. 


\section{CHAPTER 7}

\section{CONCLUSION AND FUTURE WORK}

The world is committed to reducing carbon emissions by integrating more power generated by renewable energy resources such as solar and wind. However, the variable nature of these resources poses challenges to the stability of the electrical grid. The new smart-grid device, the Residential Static VAR Compensator (RSVC), presented in this dissertation provides a solution to mitigate the unstable nature of the distributed energy resources that are powered by renewable resources. The electrical grids which contain RSVCs distributed across the distribution feeder can help to solve a variety of problems that are typical to the distribution feeder.

Before reviewing the potential applications and future work, the novel features of RSVC that set it apart from the traditional shunt devices are summarised below:

1. The RSVC can provide variable reactive power to counter the needs of a modern distribution system.

2. The RSVC is a single-phase PWM-based shunt compensation device that has minimal harmonics rather than a three-phase thyristor-control reactor-based Static VAR compensator which requires harmonic filtering for its operation.

3. Unlike conventional Static VAR Compensators, the RSVC does not require a synchronization unit (generally a phase-locked loop) for its operation. The RSVC oper- 
ates using the on switching signals that are generated based on the sign of the voltage across the switches.

4. The RSVC can provide a sub-cycle variable reactive power. This is because the switching frequency, for the bi-directional switches involved in the current commutation, is much higher than the system frequency which is generally $50 \mathrm{~Hz}$ or 60 $\mathrm{Hz}$.

5. The RSVC is a scalable device. The reactive components for the RSVC can be scaled to match the needs as required.

6. The control scheme for the voltage and power regulation loop presented in the dissertation can be used to track the desired reference voltage. This makes the RSVC devices as tools to add smartness at the periphery of the electrical grid.

7. The power regulation control loop in the closed-loop design of the RSVC implements "smart loads", i.e., the loads track their minimum power and operate at the minimum power but still providing the full performance to the end-customer.

\subsection{Applications for Deploying the RSVC}

The RSVC is a promising smart-grid technology that can be used in many applications to solve the power quality challenges that the electrical utility may face. A few of these applications are outlined below which also lists the literature that has been published (or currently being reviewed). The RSVC work presented in this dissertation is part of the United States Patent [76]. 


\subsubsection{Conservation of Power by Voltage Reduction (CVR)}

The RSVC was conceived as a device that can regulate residential voltages such that residential voltages can be reduced within the ANSI standards during peak-demand hours. It facilitates electric utilities to conserve electrical power during peak-demand hours. By reducing feeder voltages, especially during peak-demand hours, utilities will benefit through lower energy losses and lower peak generation capacity. Although CVR holds great potential in saving energy, it has been overlooked due to problems associated with cost, implementation, and loads which border on undervoltage violations during peak loading hours. The paper [77] shows the energy-saving capability of the RSVC elements when deployed to the residential locations exhibiting undervoltage violations during the reduction of the substation feeder head voltage.

\subsubsection{Mitigation of Voltage Violation}

Results presented in [78] show that the use of RSVC devices on the low side of the service transformers is an efficient way to mitigate low and high-voltage violations in the distribution feeder. During a one-month evaluation of the distribution system where RSVC devices were deployed across the feeder, the results show that the use of RSVCs was able to eliminate all voltage violations below $113 \mathrm{~V}$ and it reduced the number of voltage violations under $114 \mathrm{~V}$ by $88 \%$.

Moreover, for another feeder, the deployment of RSVCs in a distribution feeder with high penetration of power from photovoltaic arrays was able to mitigate high and low voltage experienced in the feeder. The LSF (loss-sensitivity factor) was used to determine the buses able to host an RSVC and effectively mitigating all voltage violations. 


\subsubsection{Distribution Systems Assets Deferral}

Several ways have been identified for deferring equipment upgrades such as an accurate forecast of load growth, CVR, demand response management, and battery storage. However, finding cost-effective asset deferral strategies has proven difficult because the few options available are either difficult to implement or are still too expensive. Most of the literature focuses on deferring asset replacement driven by an anticipated peak load exceeding the nameplate rating. The RSVC can be used as an alternative method for assets deferral. Chapter 6 and the paper [78] explain the utilization of the RSVC for assets deferral which results in cost saving for an electric utility.

\subsubsection{Peak Shaving During Peak-Demand Hours}

Recently, the Battery Energy Storage System (BESS) has become a popular device for peak shaving. However, BESS are limited in operation (a few hours in a day) and require maintenance. Once the state-of-charge of the BESS drops, they will not be sufficient to meet the peak-demand and the feeder will experience the same peak load as before. The RSVC can become a potential demand-response tool for utilities which does not have a limitation of charge storage for the batteries.

\subsubsection{Power Factor Correction}

Power factor correction can be achieved by using the RSVC devices. Electric utilities generally require industrial loads with poor power factor to install power factor correction units. Common power factor correction units include capacitor banks and an unloaded synchronous motor. However, both of these methods have their pros and cons. Capacitors in capacitor banks can only be switched in discrete steps whereas synchronous condensers 
require mechanical maintenance. RSVC provides a power-electronics based solution to compensate for the power factor for the industrial loads (or loads with low power factor). The amount of reactive power generated can be adjusted continuously and being a solidstate electronic device, RSVC requires less maintenance.

\subsubsection{Increasing the Hosting Capacity}

Hosting Capacity in distribution feeders can be increased with the deployment of RSVCs. The most common limit on the integration of the DERs in distribution systems is high voltage violations and voltage deviation. The RSVC can reduce the voltage at the PCC (point of common coupling), thus allowing the interconnection of more DER in the system and increasing the hosting capacity.

\subsection{Suggested Future Work}

The dissertation provides a lab-prototype for the RSVC. Although this technology has shown promising results in solving power quality issues, yet several avenues need to be explored before RSVC devices can become viable devices and physically be deployed in the distribution system. The following are some of the directions that can be taken up for further research and exploration.

\section{A Robust and Compact RSVC Design}

The lab-prototype of RSVC was tested at $60 \mathrm{Vrms}(73 \mathrm{Vrms}$ with fixed capacitors) due to the limitations of the components available in the lab. Higher power and more compact design of RSVC can be conceived and tested following the method presented in this 
dissertation. Some suggestions are to use the following components from the Semikron [39]:

Table 7.1: RSVC Suggested Power Electronics Components For Future Designs (labprototype).

\begin{tabular}{|c|c|}
\hline $\begin{array}{c}\text { Power Electronics } \\
\text { Components }\end{array}$ & Devices \\
\hline \hline Gate Driver & SKYPER_32PRO_R \\
\hline Gate Driver Core & Board_1_SKYPER_32PRO_R \\
\hline Bi-directional Switches & SKM150GM12T4G \\
\hline
\end{tabular}

\section{Development of Information and Communication Technology (ICT)}

The presented design of the RSVC does not include a mechanism to be controlled by a central controller. Such communication technology will allow system operators to enable or disable the control mechanism for the RSVCs remotely. Also when no reactive power is required from the RSVC devices, the system operators can make the RSVC operate at a duty cycle where the reactive power from the fixed capacitor and switched inductor cancel each other.

\section{Effects of Loads on RSVC Performance}

The lab-prototype of RSVC was only tested for the resistive load. The power for the resistive load varies linearly with the voltage at its terminals. It is required to further test the performance of the control mechanism with dynamic loads such as loads that utilize more power when the voltage is reduced and composite loads that include different kinds of loads. 


\section{Effects of Frequency Fluctuations on RSVC Performance}

During the development and testing of the RSVC, it has been assumed that the frequency of the system remains constant. However, DERs systems have a high susceptibility to frequency variation. More research is required to verify the behavior of the RSVC under frequency variations.

\section{Interaction of Multiple RSVC with Distribution Network}

The dynamic interaction between RSVC devices and other voltage control devices that are already deployed in the distribution network is an important consideration before installing the RSVC devices at the distribution power system.

\section{Evaluation of the Cost-Benefit for the RSVC deployment on a Realistic Feeder}

Chapter 6 presents the cost-saving by deploying the RSVC on an IEEE-13 node feeder and a realistic feeder in the north-west of the United States. However, the cost-benefit analysis only included the cost associated with the development of the laboratory prototype of RSVC. Other factors should be accounted for while making the cost-benefit analysis such as the cost associated with the development of the final commercial product and the maintenance cost (if any). It is believed that the cost of the RSVC development will be significantly lower than other commercial devices that provide capacitive reactive power to the distribution system.

\section{Optimizing the Size of the Reactive Components in the RSVC Design}

The net reactive power for the RSVC mainly depends on the inductive reactive provided by the PWM-based switched inductor. The reactive power from the switched inductor 
depends on the duty cycle at which the inductor is switching. The sizes and footprint for the inductors can be optimized by choosing the range of the reactive power requirements from the switched reactor.

\section{Comparison with Electric Springs}

Recently, Electric Spring (ES) has been proposed as a new smart-grid technology for providing voltage regulation in the presence of a variable renewable resources [79-81]. A detailed comparison between RVSC and ES is required to quantify the merits and demerits for both reactive power compensation techniques.

In conclusion, the Residential Static VAR Compensator has the potential for a novel smart-grid device. The dissertation has outlined a pathway for designing the RSVC that is supported by simulations and laboratory prototype results. A completely new approach for load management by using the control schemes (developed in the dissertation) is also presented. The RSVC devices can be used in multiple applications such as continuous voltage control at a load point, power factor control, and mitigation of power quality issues. In a nutshell, RSVC device has the potential to be a cost-effective solution for improving power quality at the distribution side of the power system. 


\section{REFERENCES}

[1] OECD, Renewables Information 2018, 2018. [Online]. Available: https://www. oecd-ilibrary.org/content/publication/renew-2018-en

[2] Vibrant Clean Energy, The Coal Cost Crossover: Economic Viability of Existing Coal Compared to new Local and Solar Resources, Mar. 2019. [Online]. Available: https://energyinnovation.org/wp-content/uploads/2019/ 04/Coal-Cost-Crossover_Energy-Innovation_VCE_FINAL2.pdf

[3] International Energy Agency, World Energy Outlook 2018, 2018. [Online]. Available: https://www.oecd-ilibrary.org/content/publication/weo-2018-en

[4] Y. Wan, "Analysis of Wind Power Ramping Behavior in ERCOT," National Renewable Energy Laboratory (NREL), Tech. Rep. TP-5500-49218, Mar. 2011.

[5] C. Voyant et al., "Machine Learning Methods for Solar Radiation Forecasting: A Review," Renewable Energy, vol. 105, pp. 569 - 582, 2017. [Online]. Available: http://www.sciencedirect.com/science/article/pii/S0960148116311648

[6] Wen-Yeau Chang, "A Literature Review of Wind Forecasting Methods," Journal of Power and Energy Engineering, vol. 2, pp. 161-168, Dec. 2013.

[7] ANSI, Electric Power Systems And Equipment - Voltage Ratings (60 Hertz). [Online]. Available: https://webstore.ansi.org/standards/nema/ansic842011

[8] R. M. Mathur and R. K. Varma, Thyristor-Based FACTS Controllers for Electrical Transmission Systems. Wiley-IEEE Press, 2002.

[9] N. G. Hingorani and L. Gyugyi, Understanding FACTS: Concepts and Technology of Flexible AC Transmission Systems. Wiley-IEEE Press, 2000.

[10] D. Coates, "FACTS: A Transmission Utility Perspective," in Proceedings of IEE Colloquium Flexible AC Transmission Systems - The FACTS, Nov. 1998, pp. 2/1-2/7.

[11] J. C. Erickson and S. R. Gilligan, "The Effects of Voltage Reduction on Distribution Circuit Loads," IEEE Transactions on Power Apparatus and Systems, vol. PAS-101, no. 7, pp. 2014-2018, Jul. 1982. 
[12] V. J. Warnock and T. L. Kirkpatrick, "Impact of Voltage Reduction on Energy and Demand: Phase II," IEEE Transactions on Power Systems, vol. 1, no. 2, pp. 92-95, May 1986.

[13] R. F. Preiss and V. J. Warnock, "Impact of Voltage Reduction on Energy and Demand," IEEE Transactions on Power Apparatus and Systems, vol. PAS-97, no. 5, pp. 1665-1671, Sep. 1978.

[14] Z. Wang and J. Wang, "Review on Implementation and Assessment of Conservation Voltage Reduction," IEEE Transactions on Power Systems, vol. 29, no. 3, pp. 13061315, May 2014.

[15] D. Kirshner, "Implementation of Conservation Voltage Reduction at Commonwealth Edison," IEEE Transactions on Power Systems, vol. 5, no. 4, pp. 1178-1182, Nov. 1990.

[16] J. G. De Steese, S. B. Merrick, and B. W. Kennedy, "Estimating methodology for a large regional application of conservation voltage reduction," IEEE Transactions on Power Systems, vol. 5, no. 3, pp. 862-870, Aug. 1990.

[17] R. F. Preiss and V. J. Warnock, "Impact of Voltage Reduction on Energy and Demand," IEEE Transactions on Power Apparatus and Systems, vol. PAS-97, no. 5, pp. 1665-1671, Sep. 1978.

[18] B. R. Williams, "Distribution Capacitor Automation Provides Integrated Control of Customer Voltage Levels and Distribution Reactive Power Flow," in Proceedings of Power Industry Computer Applications Conference, May 1995, pp. 215-220.

[19] B. W. Kennedy and R. H. Fletcher, "Conservation Voltage Reduction (CVR) at Snohomish County PUD," IEEE Transactions on Power Systems, vol. 6, no. 3, pp. 986-998, Aug. 1991.

[20] S. Lefebvre et al., "Measuring the Efficiency of Voltage Reduction at Hydro-Québec Distribution," in Proceedings of IEEE Power and Energy Society General Meeting Conversion and Delivery of Electrical Energy in the 21st Century, Jul. 2008, pp. 1-7.

[21] T. A. Short and R. W. Mee, "Voltage Reduction Field Trials on Distributions Circuits," in Proceedings of IEEE PES T\&D 2012, May 2012, pp. 1-6.

[22] T. J. Krupa and H. Asgeirsson, "The Effects of Reduced Voltage on Distribution Circuit Loads," IEEE Transactions on Power Systems, vol. 2, no. 4, pp. 1013-1018, Nov. 1987. 
[23] C. Concordia and S. Ihara, "Load Representation in Power System Stability Studies," IEEE Transactions on Power Apparatus and Systems, vol. PAS-101, no. 4, pp. 969977, Apr. 1982.

[24] R. H. Fletcher and A. Saeed, "Integrating Engineering and Economic Analysis for Conservation Voltage Reduction," in Proceedings of IEEE Power Engineering Society Summer Meeting, vol. 2, Jul. 2002, pp. 725-730.

[25] Liang Du, Lijun He, and R. G. Harley, "A Survey of Methods for Placing Shunt Capacitor Banks in Power Network with Harmonic Distortion," in Proceedings of IECON 2012 - 38th Annual Conference on IEEE Industrial Electronics Society, Oct. 2012, pp. 1198-1203.

[26] B. Milosevic and M. Begovic, "Capacitor Placement for Conservative Voltage Reduction on Distribution Feeders," IEEE Transactions on Power Delivery, vol. 19, no. 3, pp. 1360-1367, Jul. 2004.

[27] Varentec, Engo Varentec. [Online]. Available: http://varentec.com/products/ engo-v10/

[28] SVC-20 Gridco Systems, Gridco Systems. [Online]. Available: https: //d3pcsg2wjq9izr.cloudfront.net/files/93836/download/710171/6.pdf

[29] Utilidata, Saving MegaWatts with Voltage Optimization. [Online]. Available: https://utilidata.com/casestudy/saving-megawatts-with-voltage-optimization/

[30] R. Neal, "The use of AMI meters and solar PV inverters in an advanced Volt/VAr control system on a distribution circuit," in Proceedings of IEEE PES T\&D 2010, Apr. 2010, pp. 1-4.

[31] R. C. Belvin and T. A. Short, "Voltage Reduction Results on a 24-kV Circuit," in Proceedings of PES T\&D 2012, May 2012, pp. 1-4.

[32] W. B. Zhao, Y. Tang and Q. Wang, "Modeling of Common Load Components in Power System Based on Dynamic Simulation Experiments," in Proceedings of 2010 International Conference on Power System Technology, Oct. 2010, pp. 1-7.

[33] K.P. Schneider, F.K. Tuffner, J.C. Fuller and R. Singh, "Evaluation of Conservation Voltage Reduction (CVR) on a National Level," Pacific Northwest National Laboratory, Tech. Rep. PNNL-19596, Jul. 2010.

[34] A. Bokhari et al., "Experimental Determination of the ZIP Coefficients for Modern Residential, Commercial, and Industrial Loads," IEEE Transactions on Power Delivery, vol. 29, no. 3, pp. 1372-1381, Jun. 2014. 
[35] Latif, Muhammad Kamran, "Hardware Realization of a Residential Static VAR Compensator," Master's thesis, Boise State University, Boise State University Theses and Dissertations. 1265., 2017.

[36] H. Jin, G. Goos, and L. Lopes, "An Efficient Switched-Reactor-Based Static VAr Compensator," IEEE Transactions on Industry Applications, vol. 30, no. 4, pp. 9981005, Jul./Aug. 1994.

[37] Shuming Xu et al., "Bidirectional LIGBT on SOI Substrate With High Frequency and High Temperature Capability," in Proceedings of 9th International Symposium on Power Semiconductor Devices and IC's, May 1997, pp. 37-40.

[38] J. D. P. Ying-Keung Leung, A. K. Paul and S. S. Wong, "Lateral IGBT in Thin SOI for High Voltage, High Speed Power IC," IEEE Transactions on Electron Devices, vol. 45, no. 10, pp. 2251-2254, Oct. 1998.

[39] Semikron, SKM150GM12T4G. [Online]. Available: https://www.semikron.com/ products/product-classes/igbt-modules/detail/skm150gm12t4g-22892460.html

[40] S. Bernet, T. Matsuo, and T. A. Lipo, "A Matrix Converter using Reverse Blocking NPT-IGBTs and Optimized Pulse Patterns," in Proceedings of Power Electronics Specialists Conference, vol. 1, Jun. 1996, pp. 107-113.

[41] A. Alesina and M. G. B. Venturini, "Analysis and Design of Optimum-Amplitude Nine-Switch Direct AC-AC Converters," IEEE Transactions on Power Electronics, vol. 4, no. 1, pp. 101-112, Jan. 1989.

[42] C. Klumpner et al., "New steps towards a low-cost power electronic building block for matrix converters," in Proceedings of IEEE Industry Applications Conference, vol. 3, 2000, pp. 1964-1971.

[43] P. D. Ziogas, S. I. Khan, and M. H. Rashid, "Analysis and Design of Forced Commutated Cycloconverter Structures with Improved Transfer Characteristics," in Proceedings of IEEE Power Electronics Specialists Conference, Jun. 1985, pp. 610-622.

[44] C. L. Neft and C. D. Schauder, "Theory and Design of a 30-HP Matrix Converter," in Proceedings of Industry Applications Society Annual Meeting, Oct. 1988, pp. 934939.

[45] M. Ziegler and W. Hofmann, "Semi natural two steps commutation strategy for matrix converters," in Proceedings of IEEE Power Electronics Specialists Conference, vol. 1, May 1998, pp. 727-731. 
[46] L. Empringham, P. W. Wheeler, and J. C. Clare, "Intelligent Commutation of Matrix Converter Bi-Directional Switch Cells Using Novel Gate Drive Techniques," in Proceedings of IEEE Power Electronics Specialists Conference, vol. 1, 1998, pp. 707-713.

[47] B. H. Kwon, B. D. Min, and J. H. Kim, "Novel Commutation Technique of AC-AC Converters," IEE Proceedings - Electric Power Applications, vol. 145, no. 4, pp. 295300, Jul. 1998.

[48] C. Klumpner et al., "A New Matrix Converter Motor (MCM) for Industry Applications," IEEE Transactions on Industrial Electronics, vol. 49, no. 2, pp. 325-335, Apr. 2002.

[49] J. L. Galvez et al., "Intelligent Bidirectional Power Switch Module for Matrix Converter Applications," in Proceedings of IEEE Power Electronics and Applications, Sept. 2007, pp. 1-9.

[50] P. W. Wheeler et al., "Matrix converters: A Technology Review," IEEE Transactions on Industrial Electronics, vol. 49, no. 2, pp. 276-288, Apr. 2002.

[51] L. Empringham, P. Wheeler, and J. Clare, "A Matrix Converter Induction Motor Drive Using Intelligent Gate Drive Level Current Commutation Techniques," in Proceedings of Industry Applications Conference, vol. 3, 2000, pp. 1936-1941.

[52] J. Mahlein et al., "Matrix converter commutation strategies with and without explicit input voltage sign measurement," IEEE Transactions on Industrial Electronics, vol. 49, no. 2, pp. 407-414, Apr. 2002.

[53] J. Seok, K. Kim, and D. Lee, "Automatic Mode Switching of P/PI Speed Control for Industry Servo Drives Using Online Spectrum Analysis of Torque Command," IEEE Transactions on Industrial Electronics, vol. 54, no. 5, pp. 2642-2647, Oct. 2007.

[54] K. Åström and T. Hägglund, Advanced PID Control. ISA - The Instrumentation, Systems and Automation Society, 2006. [Online]. Available: http://lup.lub.lu.se/ record $/ 535630$

[55] X. lan Li, J.-G. Park, and H.-B. Shin, "Comparison and Evaluation of Anti-Windup PI Controllers," Journal of Power Electronics, vol. 11, no. 1, pp. 45 - 50, Jan. 2011.

[56] P. Huynh and B. H. Cho, "Design and Analysis of a Microprocessor-Controlled PeakPower-Tracking System [for solar cell arrays]," IEEE Transactions on Aerospace and Electronic Systems, vol. 32, no. 1, pp. 182-190, Jan. 1996. 
[57] Mao-Lin Chiang, Chih-Chiang Hua, and Jong-Rong Lin, "Direct Power Control for Distributed PV Power System," in Proceedings of the Power Conversion ConferenceOsaka, vol. 1, Apr. 2002, pp. 311-315.

[58] Weidong Xiao and W. G. Dunford, "A Modified Adaptive Hill Climbing MPPT Method for Photovoltaic Power Systems," in Proceedings of IEEE 35th Annual Power Electronics Specialists Conference, vol. 3, Jun. 2004, pp. 1957-1963.

[59] A. Pandey, N. Dasgupta, and A. K. Mukerjee, "Design Issues in Implementing MPPT for Improved Tracking and Dynamic Performance," in Proceedings of IECON 2006 32nd Annual Conference on IEEE Industrial Electronics, Nov. 2006, pp. 4387-4391.

[60] F. Liu et al., "A Variable Step Size INC MPPT Method for PV Systems," IEEE Transactions on Industrial Electronics, vol. 55, no. 7, pp. 2622-2628, Jul. 2008.

[61] N. Femia et al., "Optimization of Perturb and Observe Maximum Power Point Tracking Method," IEEE Transactions on Power Electronics, vol. 20, no. 4, pp. 963-973, Jul. 2005.

[62] D. Sera et al., "Optimized Maximum Power Point Tracker for Fast-Changing Environmental Conditions," IEEE Transactions on Industrial Electronics, vol. 55, no. 7, pp. 2629-2637, Jul. 2008.

[63] T. Noguchi, S. Togashi, and R. Nakamoto, "Short-Current Pulse-Based MaximumPower-Point Tracking Method for Multiple Photovoltaic-and-Converter Module System," IEEE Transactions on Industrial Electronics, vol. 49, no. 1, pp. 217-223, Feb. 2002.

[64] K. Irisawa et al., "Maximum Power Point Tracking Control of Photovoltaic Generation System Under Non-Uniform Insolation by Means of Monitoring Cells," in Proceedings of the 28th IEEE Photovoltaic Specialists Conference, Sep. 2000, pp. 1707-1710.

[65] ZedBoard ${ }^{\mathrm{TM}}$, ZedBoard. [Online]. Available: http://zedboard.org/product/zedboard

[66] Digilent, Pmod ENC: Rotary Encoder. [Online]. Available: https://store.digilentinc. com/pmod-enc-rotary-encoder/

[67] Infineon, IRGP4066DPbF - Insulated Gate Bipolar Transistor with Ultrafast Soft Recovery Diode. [Online]. Available: https://www.infineon.com/dgdl/Infineon-IRGP4066D-DataSheet-v01_00-EN. pdf?fileId=5546d462533600a401535655feed2454 
[68] Maxim Integrated, SANTA FE (MAXREFDES5\#): 16-Bit High Accuracy Multi-input Isolated Analog Front End (AFE). [Online]. Available: https://www.maximintegrated. com/en/design/reference-design-center/system-board/5561.html

[69] California Public Utility Commissions, What are TOU rates? [Online]. Available: https://www.cpuc.ca.gov/General.aspx?id=12194

[70] J. Leadbetter and L. Swan, "Battery Storage System for Residential Electricity Peak Demand Shaving," Energy and Buildings, vol. 55, pp. 685 - 692, 2012.

[71] A. J. Pimm, T. T. Cockerill, and P. G. Taylor, "The Potential for Peak Shaving on Low Voltage Distribution Networks Using Electricity Storage," Journal of Energy Storage, vol. 16, pp. $231-242,2018$.

[72] U. Prasatsap, S. Kiravittaya, and J. Polprasert, "Determination of Optimal Energy Storage System for Peak Shaving to Reduce Electricity Cost in a University," Energy Procedia, vol. 138, pp. 967 - 972, 2017.

[73] W. A. Qureshi, N.-K. C. Nair, and M. M. Farid, "Impact of Eenergy Storage in Buildings on Electricity Demand Side Management," Energy Conversion and Management, vol. 52, no. 5, pp. $2110-2120,2011$.

[74] H. L. Willis, Power Distribution Planning Reference Book. Marcel Dekker, Inc., 2004.

[75] Lazard, Lazard's Levelized Cost Of Storage Analysis - Version 4.0, Nov. 2018. [Online]. Available: https://www.lazard.com/media/450774/ lazards-levelized-cost-of-storage-version-40-vfinal.pdf

[76] S. Ahmed-Zaid and M. K. Latif, "Residential Static VAR Compensator Apparatus and Method," May 2, 2019, US Patent App. 15/798,675.

[77] J. P. Stubban, S. Ahmed-Zaid, and M. K. Latif, "Conservation by Voltage Reduction Using a Single-Phase Residential Static VAR Compensator," in Proceedings of Clemson University Power Systems Conference (PSC), Sep. 2018, pp. 1-6.

[78] A. V. Delgado, M. Kamran Latif, and S. Ahmed-Zaid, "Mitigation of Distribution Voltage Violations Using Single-Phase Residential Static VAr Compensators," in Proceedings of IEEE 62nd International Midwest Symposium on Circuits and Systems (MWSCAS), Aug. 2019, pp. 525-528.

[79] S. Y. Hui, C. K. Lee, and F. F. Wu, "Electric SpringsA New Smart Grid Technology," IEEE Transactions on Smart Grid, vol. 3, no. 3, pp. 1552-1561, Sep. 2012. 
[80] C. K. Lee, B. Chaudhuri, and S. Y. Hui, "Hardware and Control Implementation of Electric Springs for Stabilizing Future Smart Grid With Intermittent Renewable Energy Sources," IEEE Journal of Emerging and Selected Topics in Power Electronics, vol. 1, no. 1, pp. 18-27, Mar. 2013.

[81] J. Soni and S. K. Panda, "Electric Spring for Voltage and Power Stability and Power Factor Correction," IEEE Transactions on Industry Applications, vol. 53, no. 4, pp. 3871-3879, Jul. 2017. 


\section{APPENDIX A}




\section{FOURIER SERIES REPRESENTATION OF A PULSE FUNCTION}

Consider the periodic pulse function in Figure A.1. It is an even function with period $T$. The function is a pulse with an amplitude of 1 and with a pulse width of $T_{p}$. The function can be defined over one period (having center at the origin) as:

$$
x_{T}(t)=\left\{\begin{array}{lll}
1 & \text { for } \quad 0 \leq t \leq T_{p} / 2 \\
0 & \text { for } \quad T_{p} / 2 \leq t \leq T / 2
\end{array}\right.
$$

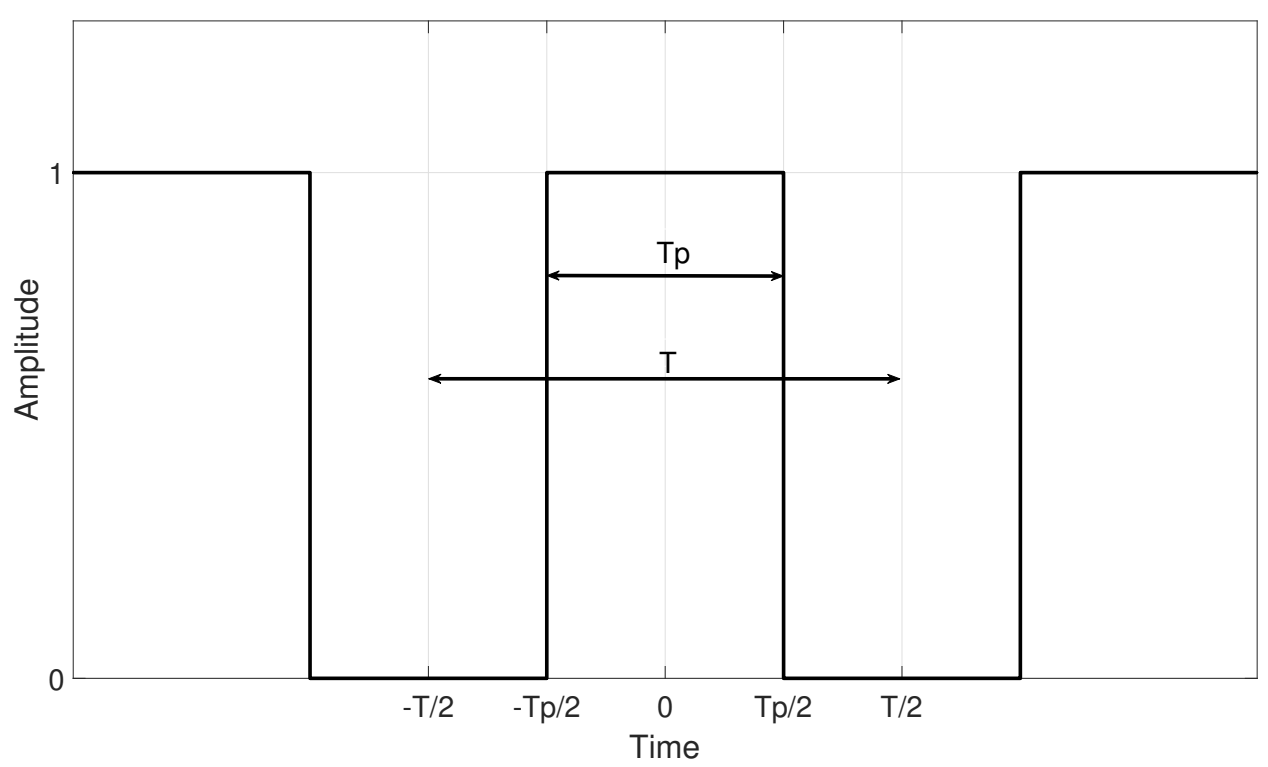

Figure A.1: Periodic Pulse Function with an Amplitude of 1, time period of $\mathrm{T}$ and pulse width of $T_{p}$

The Fourier decomposition of any regular time-varying waveform $f(t)$ can be expressed as an infinite series of sinusoidal harmonics i.e. 


$$
f(t)=a_{0}+\sum_{n=1}^{\infty}\left(a_{n} \cos n \omega_{o} t+b_{n} \sin n \omega_{o} t\right)
$$

where

$$
\begin{aligned}
& a_{0}=\frac{1}{T} \int_{0}^{T} f(t) d t \\
& a_{n}=\frac{2}{T} \int_{0}^{T} f(t) \cos n \omega_{o} t d t \\
& b_{n}=\frac{2}{T} \int_{0}^{T} f(t) \sin n \omega_{o} t d t
\end{aligned}
$$

Since the pulse function is an even function, therefore there are only $a_{n}$ terms. The average value of the function can be easily found

$$
a_{0}=\frac{T_{p}}{T}=D
$$

where D is the duty cycle of the pulse function. The other terms follow from

$$
a_{n}=\frac{2}{T} \int_{0}^{T} x_{t}(t) \cos n \omega_{o} t d t, \quad n \neq 0
$$

We can consider any interval of one period but the interval from $-T / 2$ to $T / 2$ is straightforward in this case i.e.

$$
a_{n}=\frac{2}{T} \int_{\frac{-T}{2}}^{\frac{T}{2}} x_{t}(t) \cos n \omega_{o} t d t, \quad n \neq 0
$$

Since $x_{t}(t)=1$ only between $-T_{p} / 2$ to $T_{p} / 2$ the integral can be simplified as follow 


$$
\begin{aligned}
a_{n} & =\frac{2}{T} \int_{\frac{-T_{p}}{2}}^{\frac{T_{p}}{2}} \cos n \omega_{o} t d t=\left.\frac{2}{T} \frac{1}{n \omega_{o}} \sin n \omega_{o} t\right|_{\frac{-T_{p}}{2}} ^{\frac{T_{p}}{2}} \\
& =\frac{2}{T} \frac{1}{n \omega_{o}}\left(\sin \left(n \omega_{o} \frac{T_{p}}{2}\right)-\sin \left(-n \omega_{o} \frac{T_{p}}{2}\right)\right) \\
& =\frac{4}{T} \frac{1}{n \omega_{o}} \sin \left(n \omega_{o} \frac{T_{p}}{2}\right)
\end{aligned}
$$

Since, sine is an odd function, $\sin (x)-\sin (-x)=2 \sin (x)$, and also using the fact that $\omega_{0}=2 \pi / T$ then

$$
a_{n}=\frac{2}{n \pi} \sin \left(n \pi \frac{T_{p}}{T}\right)=\frac{2}{n \pi} \sin (n \pi D)
$$

Therefore, the complete fourier series transform of a pulse signal is given as

$$
f(t)=a_{0}+\sum_{n=1}^{\infty}\left(a_{n} \cos n \omega_{o} t\right)=D+\sum_{n=1}^{\infty} \frac{2}{n \pi} \sin (n \pi D) \cos \left(n \omega_{o} t\right)
$$

Thus Equation (A.4) shows the fourier series transform of a pulse function. 